Supplementary Information for

\title{
Telechelic Helical Poly(quinoxaline-2,3-diyl)s Containing a Structurally Defined, Circularly Polarized Luminescent Terquinoxaline Core: Synthesis by Core-initiated Bidirectional Living Polymerization
}

\author{
Shogo Kuriyama, Yuuya Nagata, and Michinori Suginome*
}

Department of Synthetic Chemistry and Biological Chemistry, Graduate School of Engineering, Kyoto University, Kyoto 606-8501, Japan

$$
\text { 615-8510, Japan }
$$

*To whom correspondence should be addressed.

E-mail: suginome@sbchem.kyoto-u.ac.jp

\section{Contents}

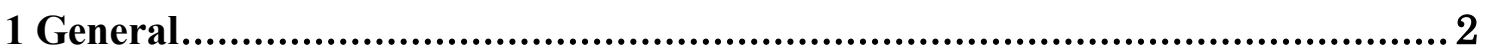

2 Experimental Procedures and Spectral Data for New Compounds .................. 4

3 Characterization of 1 in solution .................................................... 19

4 MALDI-TOF-MS and ${ }^{1}$ H NMR Analysis of the Polymers ......................... 21

5 Chiroptical Properties of the Polymers............................................... 23

6 Fluorescent Properties of Terquinoxalines 2 .............................................25

7 Circularly Polarized Luminescence (CPL) Measurement .............................26

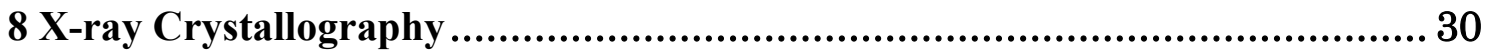

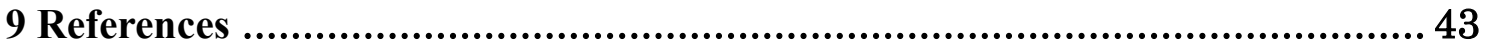

10 UV-vis, CD, and PL Spectra of New Compounds ................................ 44

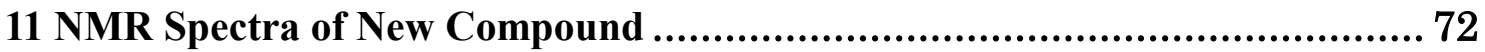




\section{General}

All reactions were carried out under an atmosphere of nitrogen with magnetic stirring. ${ }^{1} \mathrm{H}$ NMR spectra were recorded on a Varian 400-MR (399.89 MHz) spectrometer at ambient temperature. ${ }^{13} \mathrm{C}$ NMR spectra were recorded on Varian 400-MR (100.55 MHz) and JEOL JNM-ECA600 (150.92 MHz) spectrometers at ambient temperature. ${ }^{31} \mathrm{P}$ NMR spectra were recorded on a Varian 400-MR (161.88 MHz) spectrometer at ambient temperature. ${ }^{1} \mathrm{H}$ NMR data are reported as follows: chemical shift in ppm downfield from tetramethylsilane ( $\delta$ scale $)$, multiplicity $(\mathrm{s}=$ singlet, $\mathrm{d}=$ doublet, $\mathrm{t}=$ triplet, $\mathrm{q}=$ quartet, quint $=$ quintet, sex $=$ sextet, $\mathrm{m}=$ multiplet and $\mathrm{br}=$ broad $)$, coupling constant $(\mathrm{Hz})$, and integration. All ${ }^{13} \mathrm{C}$ NMR and ${ }^{31} \mathrm{P}$ NMR spectra were obtained with complete proton decoupling. IR spectra were obtained using a Shimadzu FTIR-8400 Fourier transform infrared (FT-IR) spectrometer equipped with PIKE MIRacle attenuated total reflection (MIR-ATR) attachment. The GPC analysis was carried out with ALPHA-M (THF, polystyrene standards). Preparative GPC was performed on JAI LC-908 equipped with JAIGEL-1H and $-2 \mathrm{H}$ columns in a series $\left(\mathrm{CHCl}_{3}\right)$. UV-vis absorption spectra were recorded on a JASCO V-750 spectrometer equipped with a JASCO ETC-505T temperature/stirring controller at $20{ }^{\circ} \mathrm{C}$. Circular dichroism (CD) spectra were recorded on a JASCO J-1500 spectrometer equipped with a JASCO PTC-510 temperature/stirring controller at $20{ }^{\circ} \mathrm{C}$. Fluorescence spectra were recorded on a JASCO FP-6300 spectrofluorometer. Absolute quantum yields were measured by a Hamamatsu absolute PL quantum yield spectrometer C11347. Circularly polarized luminescence (CPL) spectra were recorded on a JASCO CPL-200S at room temperature. Flash chromatography was performed using a Biotage Isolera One flash purification system with silica gel flash cartridges. High resolution mass spectra were recorded on Thermo Fisher Scientific EXACTIVE (ESI and APCI) and Bruker ultraflex (Matrix-assisted laser desorption ionization time of flight-mass, MALDI-TOF-MS) spectrometers. Elemental analyses were carried out in Organic Elemental Microanalysis Laboratory of Kyoto University.

Tetrahydrofurane (THF) and toluene were dried and deoxygenized using an alumina/catalyst column system (Glass Contour Co.). 1,2-bis((S)-sec-butoxymethyl)-4,5diisocyano-3,6-dimethylbenzene (M1), ${ }^{1}$ 4,4"-Dimethyl-[1,1':4',1"-terphenyl]-2',3'diamine $(\mathbf{4 a}),{ }^{2} 4,4^{\prime \prime}$-dimethoxy-[1,1':4',1"-terphenyl]-2',3'-diamine (4b), ${ }^{3}$ and 3,6-bis(3methylthiophen-2-yl)-benzene-1,2-diamine $(\mathbf{4 c})^{3}$ were prepared according to the reported 
procedures. Other chemical reagents were purchased from the commercial sources and were used without further purification. 


\section{Experimental Procedures and Spectral Data for New Compounds}

\section{Synthesis of S1}

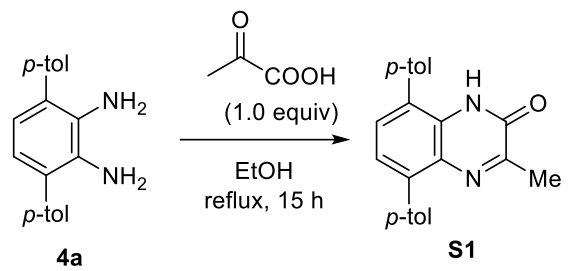

A mixture of $4 \mathbf{a}(3.75 \mathrm{~g}, 13.0 \mathrm{mmol})$ and pyruvic acid $(1.16 \mathrm{~g}, 13.2 \mathrm{mmol})$ in ethanol (60 $\mathrm{mL}$ ) was heated under reflux for $15 \mathrm{~h}$. The reaction mixture was cooled to $0{ }^{\circ} \mathrm{C}$ to afford white precipitate, which was collected by filtration, washed with cold ethanol $(6 \mathrm{~mL}, 3$ times), and dried in vacuo to give $\mathbf{S 1}$ as a white solid (2.98 g, $8.75 \mathrm{mmol}, 67 \%$ yield). ${ }^{1} \mathrm{H}$ $\operatorname{NMR}\left(\mathrm{CDCl}_{3}\right) \delta 8.87(\mathrm{~s}, 1 \mathrm{H}), 7.58-7.29(\mathrm{~m}, 10 \mathrm{H}), 2.53(\mathrm{~s}, 3 \mathrm{H}), 2.46(\mathrm{~s}, 3 \mathrm{H}), 2.45(\mathrm{~s}, 3 \mathrm{H})$; ${ }^{13} \mathrm{C} \mathrm{NMR}\left(\mathrm{CDCl}_{3}\right) \delta 158.2,154.5,139.7,138.8,137.5,135.3,132.3,130.6,130.4,130.0$, 129.8, 129.1, 128.8, 128.7, 127.4, 124.8, 21.27, 21.25, 21.0; IR (ATR, neat) $1653 \mathrm{~cm}^{-1}$ (CO); HRMS (APCI) m/z calcd for $\mathrm{C}_{23} \mathrm{H}_{21} \mathrm{~N}_{2} \mathrm{O}^{+}\left(\mathrm{M}+\mathrm{H}^{+}\right)$:341.1648, found: 341.1640.

\section{Synthesis of S2}

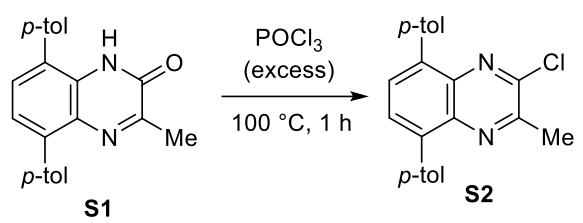

S1 $(2.98 \mathrm{~g}, 8.75 \mathrm{mmol})$ in $\mathrm{POCl}_{3}(17 \mathrm{~mL})$ was stirred at $100{ }^{\circ} \mathrm{C}$ for $1 \mathrm{~h}$. The resultant solution was poured into ice water $(300 \mathrm{~mL})$. The mixture was neutralized with $\mathrm{NaOH}$ $(30 \mathrm{~g})$ and extracted with a mixture of AcOEt $(400 \mathrm{~mL})$ and toluene $(50 \mathrm{~mL})$. Organic phase was washed with water $(100 \mathrm{~mL})$ and brine $(100 \mathrm{~mL})$ and dried over $\mathrm{MgSO}_{4}$. Filtration and evaporation of the solvent afforded $\mathbf{S 2}$ as a yellow solid (3.17 g, quant). This material was used for the next step without further purification. ${ }^{1} \mathrm{H}$ NMR $\left(\mathrm{CDCl}_{3}\right) \delta$ 7.82 (s, 2H), 7.63 (d, $J=8.0 \mathrm{~Hz}, 4 \mathrm{H}), 7.33$ (d, $J=8.0 \mathrm{~Hz}, 4 \mathrm{H}), 2.80$ (s, 3H), 2.464 (s, $3 \mathrm{H}), 2.456(\mathrm{~s}, 3 \mathrm{H}) ;{ }^{13} \mathrm{C} \mathrm{NMR}\left(\mathrm{CDCl}_{3}\right) \delta 151.7,147.0,139.2,139.0,138.82,138.79,137.6$, 137.5, 135.2, 134.9, 130.6, 130.5, 130.2, 130.0, 128.9, 128.8, 23.3, 21.3; HRMS (APCI) $\mathrm{m} / \mathrm{z}$ calcd for $\mathrm{C}_{23} \mathrm{H}_{20} \mathrm{ClN}_{2}{ }^{+}\left(\mathrm{M}+\mathrm{H}^{+}\right)$: 359.1310, found: 359.1302 .

\section{Synthesis of 5}




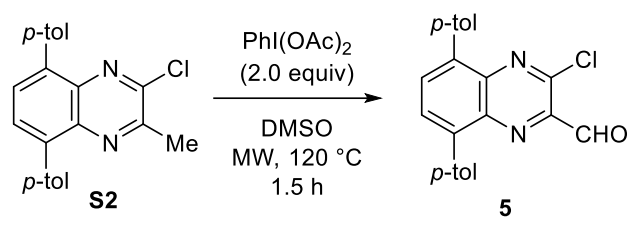

This compound was prepared from a method reported by Xie and coworkers. ${ }^{4}$ A mixture of S2 (359 mg, $1.00 \mathrm{mmol})$ and PhI(OAc) 2 (647 mg, $2.01 \mathrm{mmol})$ in DMSO (5 mL) was stirred at $120{ }^{\circ} \mathrm{C}$ for $1.5 \mathrm{~h}$ under microwave irradiation. To the reaction mixture were added sat. $\mathrm{NaHCO}_{3}$ aq. $(20 \mathrm{~mL})$ and extracted with $\mathrm{CH}_{2} \mathrm{Cl}_{2}(50 \mathrm{~mL})$ and $\mathrm{Et}_{2} \mathrm{O}(30 \mathrm{~mL})$. The combined extracts were washed with water $(10 \mathrm{~mL})$ and brine $(20 \mathrm{~mL})$ and dried over $\mathrm{MgSO}_{4}$. After filtration and evaporation of the solvent, the residue was purified with silica gel column chromatography (hexane: $\mathrm{CH}_{2} \mathrm{Cl}_{2}=75: 25$ to $0: 100$ ) to give 5 as an orange solid (263 mg, $0.704 \mathrm{mmol}, 70 \%$ yield). ${ }^{1} \mathrm{H}$ NMR $\left(\mathrm{CDCl}_{3}\right) \delta 10.24(\mathrm{~s}, 1 \mathrm{H}), 8.05$ (d, $J=7.6 \mathrm{~Hz}, 2 \mathrm{H}), 7.97$ (d, $J=7.6 \mathrm{~Hz}, 2 \mathrm{H}), 7.67-7.61(\mathrm{~m}, 4 \mathrm{H}), 7.37-7.35$ (m, 4H), 2.48 (s, 3H), 2.47 (s, 3H); ${ }^{13} \mathrm{C}$ NMR $\left(\mathrm{CDCl}_{3}\right) \delta 195.2,150.9,145.8,144.0,141.8,140.8,140.6$, 139.7, 139.6, 138.72, 138.67, 138.1, 137.8, 137.6, 137.5, 137.2, 134.5, 134.3, 134.1, 134.0, 133.5, 131.2, 130.7, 130.46, 130.45, 130.2, 129.8, 129.7, 129.04, 129.01, 128.9, 128.7, 74.0, 21.3, 21.20, 21.17; IR (ATR, neat) $1719 \mathrm{~cm}^{-1}$ (CO); HRMS (APCI) m/z calcd for $\mathrm{C}_{23} \mathrm{H}_{17} \mathrm{ClN}_{2}+\mathrm{H}^{+}\left(\mathrm{M}+\mathrm{H}^{+}\right)$: 373.1102, found: 373.1093 . 


\section{Synthesis of S3}

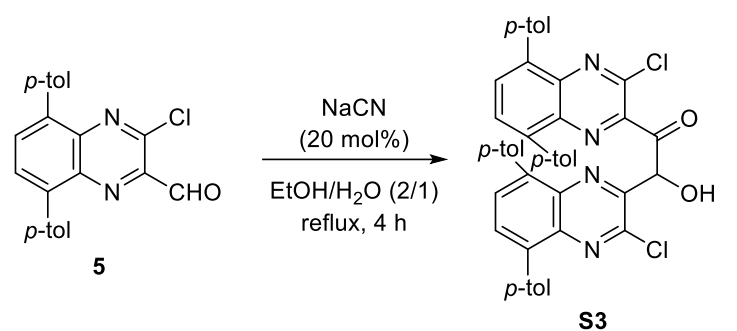

A mixture of 5 (1.67 g, $4.48 \mathrm{mmol})$ and $\mathrm{NaCN}$ (43.6 mg, $0.890 \mathrm{mmol})$ in water $(11 \mathrm{~mL})$ and $\mathrm{EtOH}(22 \mathrm{~mL})$ was heated under reflux for $4 \mathrm{~h}$. To the mixture was added water (50 $\mathrm{mL})$ to give orange precipitate. The resultant precipitate was collected by filtration, washed with water (10 mL, 2 times) and $\mathrm{EtOH}(10 \mathrm{~mL})$, and dried in vacuo give $\mathbf{S 3}$ as an orange solid (1.45 g, $1.95 \mathrm{mmol}, 87 \%$ yield). ${ }^{1} \mathrm{H} \mathrm{NMR}\left(\mathrm{CDCl}_{3}\right) \delta 7.94(\mathrm{~d}, J=7.6 \mathrm{~Hz}, 1 \mathrm{H})$, 7.82-7.77 (m, 2H), $7.70(\mathrm{~d}, J=7.6 \mathrm{~Hz}, 1 \mathrm{H}), 7.60(\mathrm{~d}, J=8.0 \mathrm{~Hz}, 4 \mathrm{H}), 7.36-7.33(\mathrm{~m}, 4 \mathrm{H})$, $7.16(\mathrm{~s}, 4 \mathrm{H}), 7.11(\mathrm{~s}, 4 \mathrm{H}), 6.70(\mathrm{~d}, J=7.6 \mathrm{~Hz}, 1 \mathrm{H}), 4.22(\mathrm{~d}, J=7.6 \mathrm{~Hz}, 1 \mathrm{H}), 2.47(\mathrm{~s}, 6 \mathrm{H})$, $2.38(\mathrm{~s}, 3 \mathrm{H}), 2.37$ (s, 3H); ${ }^{13} \mathrm{C} \mathrm{NMR}\left(\mathrm{CDCl}_{3}\right) \delta 195.2,150.9,145.7,143.9,141.7,140.8$, $140.6,139.7$, 139.6, 138.7, 138.6, 138.1, 137.8, 137.55, 137.50, 137.2, 134.5, 134.3, 134.1, 134.0, 133.5, 131.2, 130.6, 130.44, 130.43, 130.1, 129.8, 129.7, 129.02, 128.99, 128.9, 128.7, 74.0, 21.3, 21.18, 21.15.; IR (neat) $1711 \mathrm{~cm}^{-1}$ (CO); HRMS (APCI) m/z calcd for $\mathrm{C}_{46} \mathrm{H}_{34} \mathrm{Cl}_{2} \mathrm{~N}_{4} \mathrm{O}_{2}+\mathrm{H}^{+}\left(\mathrm{M}+\mathrm{H}^{+}\right)$: 745.2132, found: 745.2112.

\section{Synthesis of 3}
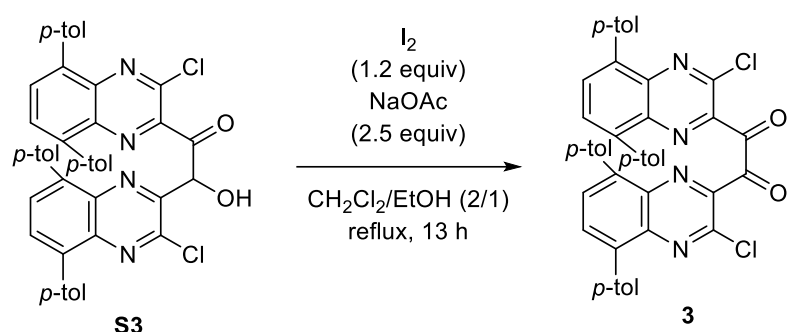

A mixture of $\mathbf{S 3}$ (1.45 g, $1.95 \mathrm{mmol})$, iodine (590 mg, $2.32 \mathrm{mmol}$ ), and $\mathrm{NaOAc}$ (398 $\mathrm{mg}$, $4.85 \mathrm{mmol})$ in $\mathrm{CH}_{2} \mathrm{Cl}_{2}(80 \mathrm{~mL})$ and $\mathrm{EtOH}(40 \mathrm{~mL})$ was heated under reflux for $13 \mathrm{~h}$. After sat. $\mathrm{Na}_{2} \mathrm{~S}_{2} \mathrm{O}_{3}$ aq $(20 \mathrm{~mL})$ added to the reaction mixture, the solvent was evaporated. The residue was washed with water $(50 \mathrm{~mL}), 1 \mathrm{M} \mathrm{NaOH}$ aq. $(20 \mathrm{~mL})$, water (20 mL, 2 times), $\mathrm{MeOH}(10 \mathrm{~mL})$, toluene/hexane (1/2, $10 \mathrm{~mL}, 3$ times), and then hexane (5 mL, 3 times) to give 3 as an orange solid $(1.22 \mathrm{~g}, 1.64 \mathrm{mmol}, 84 \%$ yield $) .{ }^{1} \mathrm{H} \mathrm{NMR}\left(\mathrm{CDCl}_{3}\right) \delta 7.97(2 \mathrm{H}$, $\mathrm{d}, J=7.6 \mathrm{~Hz}), 7.70(2 \mathrm{H}, \mathrm{d}, J=7.6 \mathrm{~Hz}), 7.66(4 \mathrm{H}, \mathrm{d}, J=8.0 \mathrm{~Hz}), 7.39(4 \mathrm{H}, \mathrm{d}, J=8.0 \mathrm{~Hz})$, $6.84(4 \mathrm{H}, \mathrm{d}, J=8.0 \mathrm{~Hz}), 6.75(4 \mathrm{H}, \mathrm{d}, J=8.0 \mathrm{~Hz}), 2.50(6 \mathrm{H}, \mathrm{s}), 2.16(6 \mathrm{H}, \mathrm{s}) ;{ }^{13} \mathrm{C} \mathrm{NMR}$ 
$\left(\mathrm{CDCl}_{3}\right) \delta 192.4,143.3,140.73 .140 .67,140.4,138.6,138.2,137.8,137.2,134.0,133.9$, 133.3, 130.4, 129.9, 129.4, 129.0, 128.0, 21.3, 21.0; IR (ATR, neat) $1717 \mathrm{~cm}^{-1}$ (CO); HRMS (APCI) m/z calcd for $\mathrm{C}_{46} \mathrm{H}_{32} \mathrm{Cl}_{2} \mathrm{~N}_{4} \mathrm{O}_{4}+\mathrm{H}^{+}\left(\mathrm{M}+\mathrm{H}^{+}\right)$: 743.1975, found: 743.1956.

\section{Synthesis of $2 a$}
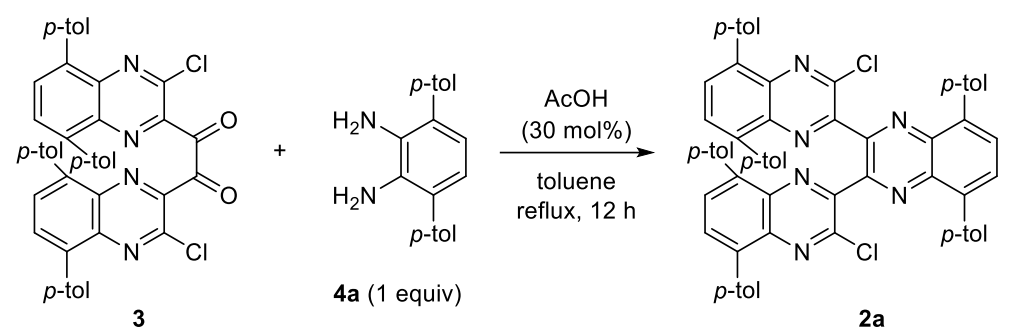

A mixture of $\mathbf{3}(231 \mathrm{mg}, 0.311 \mathrm{mmol}), \mathbf{4 a}(94.1 \mathrm{mg}, 0.326 \mathrm{mmol})$, and acetic acid (5.5 $\mathrm{mg}, 0.092 \mathrm{mmol})$ in toluene $(6.5 \mathrm{~mL})$ was heated under reflux for $12 \mathrm{~h}$. Addition of $\mathrm{MeOH}$ $(30 \mathrm{~mL})$ to the reaction mixture afforded yellow precipitate, which was collected by filtration, washed with $\mathrm{MeOH}$ ( $3 \mathrm{~mL}, 3$ times), dried in vacuo to give $\mathbf{2 a}$ as a yellow solid (277 mg, $0.278 \mathrm{mmol}, 90 \%) .{ }^{1} \mathrm{H} \mathrm{NMR}\left(\mathrm{CDCl}_{3}\right) \delta 7.98(2 \mathrm{H}, \mathrm{s}), 7.78(2 \mathrm{H}, \mathrm{d}, J=7.6 \mathrm{~Hz})$, $7.74(4 \mathrm{H}, \mathrm{d}, J=8.0 \mathrm{~Hz}), 7.61(6 \mathrm{H}, \mathrm{d}, J=8.0 \mathrm{~Hz}), 7.34(4 \mathrm{H}, \mathrm{d}, J=7.6 \mathrm{~Hz}), 7.23(4 \mathrm{H}, \mathrm{d}$, $J=7.6 \mathrm{~Hz}), 6.99(4 \mathrm{H}, \mathrm{d}, J=7.6 \mathrm{~Hz}), 6.90(4 \mathrm{H}, \mathrm{s}), 2.48(6 \mathrm{H}, \mathrm{s}), 2.40(6 \mathrm{H}, \mathrm{s}), 2.27(6 \mathrm{H}$, s); ${ }^{13} \mathrm{C} \mathrm{NMR}\left(\mathrm{CDCl}_{3}\right) \delta 148.9,148.6,145.6,140.3,139.9,139.2,138.9,138.6,137.8$, $137.6,137.5,137.2,134.83,134.78,134.4,131.3,131.2,131.0,130.5,130.1,129.6$, 128.84, 128.77, 21.30, 21.25, 21.1; HRMS (ESI) m/z calcd for $\mathrm{C}_{66} \mathrm{H}_{48} \mathrm{Cl}_{2} \mathrm{~N}_{6}+\mathrm{H}^{+}\left(\mathrm{M}+\mathrm{H}^{+}\right)$: 1017.3210, found: 1017.3182 . 


\section{Synthesis of $\mathbf{2 b}$}
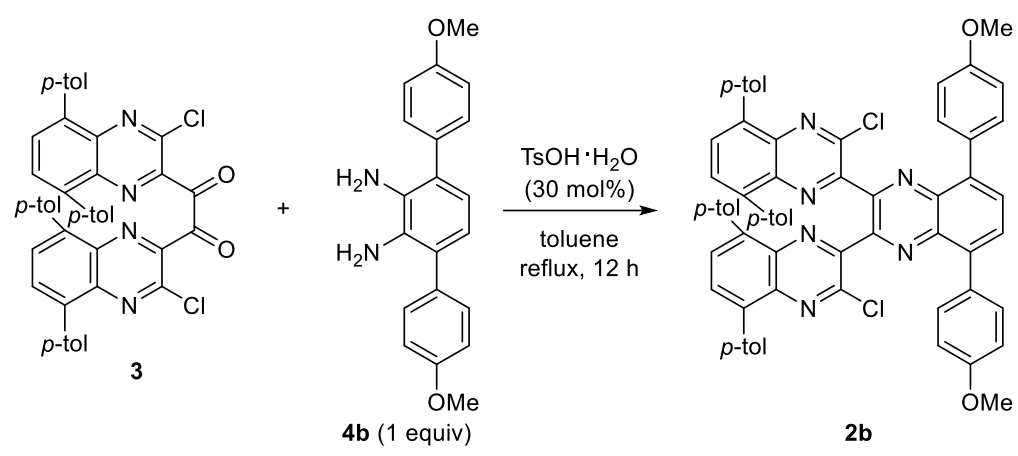

A mixture of 3 (37.2 mg, $0.050 \mathrm{mmol}), \mathbf{4 b}(16.6 \mathrm{mg}, 0.052 \mathrm{mmol})$, and $p$-toluenesulfonic acid $(\mathrm{TsOH})$ mono hydrate $(2.3 \mathrm{mg}, 0.013 \mathrm{mmol})$ in toluene $(2 \mathrm{~mL})$ was heated under reflux for $12 \mathrm{~h}$. To the reaction mixture were added sat. $\mathrm{NaHCO}_{3}$ aq. ( $3 \mathrm{~mL}$ ) and extracted with $\mathrm{CH}_{2} \mathrm{Cl}_{2}$ (10 mL, 3 times). The combined extracts were dried over $\mathrm{MgSO}_{4}$. After filtration and evaporation of the solvent, the residue was suspended in $\mathrm{CH}_{2} \mathrm{Cl}_{2}$ (2 mL). Addition of $\mathrm{MeOH}(8 \mathrm{~mL})$ afforded yellow precipitate, which was filtered, washed with $\mathrm{MeOH}$ (2 mL, 2 times), $\mathrm{Et}_{2} \mathrm{O}(2 \mathrm{~mL})$, and hexane $(1 \mathrm{~mL})$, and dired in vacuo to give $\mathbf{2 b}$ as a yellow solid (41.0 mg, $0.040 \mathrm{mmol}, 80 \%$ yield). ${ }^{1} \mathrm{H} \mathrm{NMR}\left(\mathrm{CDCl}_{3}\right) \delta 7.96(2 \mathrm{H}, \mathrm{s})$, $7.80(6 \mathrm{H}, \mathrm{d}, J=8.8 \mathrm{~Hz}), 7.64-7.60(6 \mathrm{H}, \mathrm{m}), 7.34(4 \mathrm{H}, \mathrm{d}, J=7.6 \mathrm{~Hz}), 7.00-6.91(12 \mathrm{H}, \mathrm{m})$, $3.85(6 \mathrm{H}, \mathrm{s}), 2.47(6 \mathrm{H}, \mathrm{s}), 2.24(6 \mathrm{H}, \mathrm{s}) ;{ }^{13} \mathrm{C} \mathrm{NMR}\left(\mathrm{CDCl}_{3}, 150 \mathrm{MHz}\right) \delta 159.4,148.8$, 148.6, 145.5, 139.8, 139.6, 139.2, 138.9, 138.6, 137.8, 137.7, 137.2, 134.7, 134.4, 132.2, $131.2,131.1,130.5,130.11,130.07,129.7,128.9,128.7,113.5,55.3,21.3,21.2$; HRMS (ESI) $\mathrm{m} / \mathrm{z}$ calcd for $\mathrm{C}_{66} \mathrm{H}_{48} \mathrm{Cl}_{2} \mathrm{~N}_{6} \mathrm{O}_{2}+\mathrm{H}^{+}\left(\mathrm{M}+\mathrm{H}^{+}\right)$: 1027.3289 , found: 1027.3261 . 


\section{Synthesis of $2 c$}

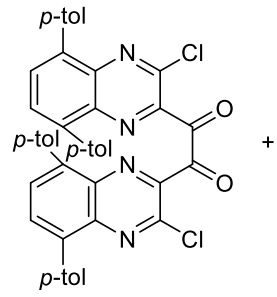

3

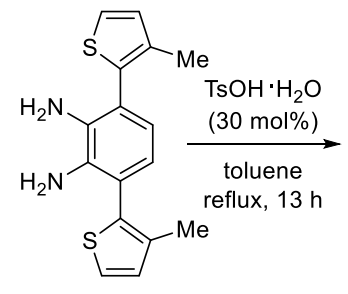

4c (1 equiv)

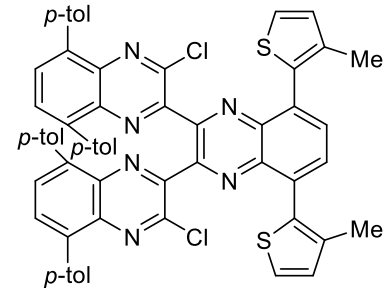

2c

A mixture of 3 (213 mg, $0.287 \mathrm{mmol}), 4 \mathbf{c}(89.9 \mathrm{mg}, 0.299 \mathrm{mmol})$, and $p$-toluenesulfonic acid mono hydrate $(14.5 \mathrm{mg}, 0.084 \mathrm{mmol})$ in toluene $(11 \mathrm{~mL})$ was heated under reflux for $13 \mathrm{~h}$. To the reaction mixture were added sat. $\mathrm{NaHCO}_{3}$ aq. $(5 \mathrm{~mL})$ and extracted with $\mathrm{CH}_{2} \mathrm{Cl}_{2}$ (10 mL, 3 times). The combined extracts were dried over $\mathrm{MgSO}_{4}$. After filtration and evaporation of the solvent, the residue was suspended in toluene $(6 \mathrm{~mL})$. Addition of $\mathrm{MeOH}(25 \mathrm{~mL})$ afforded yellow precipitate, which was filtered, washed with $\mathrm{MeOH}(4$ $\mathrm{mL}, 2$ times) and then hexane ( $4 \mathrm{~mL}, 2$ times), and dired in vacuo to give $\mathbf{2 c}$ as a yellow solid (264 mg, $0.262 \mathrm{mmol}, 91 \%$ yield). ${ }^{1} \mathrm{H}$ NMR $\left(\mathrm{CDCl}_{3}\right) \delta 7.94(2 \mathrm{H}, \mathrm{s}), 7.80(2 \mathrm{H}, \mathrm{d}, J$ $=8.4 \mathrm{~Hz}), 7.64-7.61(6 \mathrm{H}, \mathrm{m}), 7.36-7.33(6 \mathrm{H}, \mathrm{m}), 6.98-6.95(6 \mathrm{H}, \mathrm{m}), 6.86(4 \mathrm{H}, \mathrm{d}, J=7.6$ $\mathrm{Hz}), 2.47(6 \mathrm{H}, \mathrm{s}), 2.26(6 \mathrm{H}, \mathrm{s}), 2.24(6 \mathrm{H}, \mathrm{s}) .{ }^{13} \mathrm{C} \mathrm{NMR}\left(\mathrm{CDCl}_{3}\right) \delta 148.9,148.4,145.7$, 140.0, 139.6, 139.3, 138.6, 137.8, 137.6, 137.2, 136.3, 134.7, 134.3, 134.1, 132.6, 132.5, 131.2, 130.5, 130.1, 129.8, 129.6, 128.84, 128.78, 125.9, 21.3, 21.1, 15.5; HRMS (APCI) $\mathrm{m} / \mathrm{z}$ calcd for $\mathrm{C}_{62} \mathrm{H}_{44} \mathrm{Cl}_{2} \mathrm{~N}_{6} \mathrm{~S}_{2}+\mathrm{H}^{+}\left(\mathrm{M}+\mathrm{H}^{+}\right)$: 1007.2519, found: 1007.2428. 
Synthesis of $1 \mathrm{a} \cdot 0.3 \mathrm{C}_{4} \mathrm{H}_{8} \mathrm{O} \cdot 0.2 \mathrm{C}_{4} \mathrm{H}_{10} \mathrm{O}$

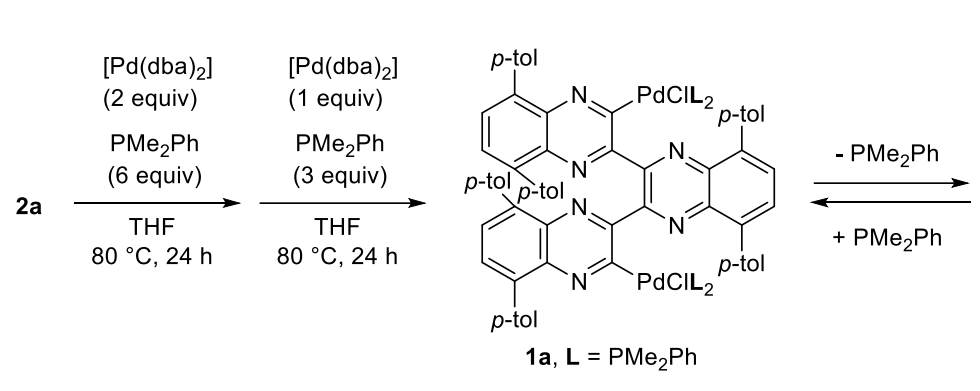

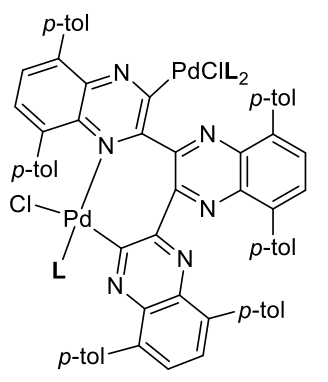

$7 a$

A mixture of $\mathbf{2 a}(99.8 \mathrm{mg}, 0.100 \mathrm{mmol}), \mathrm{Pd}(\mathrm{dba})_{2}(115.2 \mathrm{mg}, 0.200 \mathrm{mmol})$, and $\mathrm{PMe}_{2} \mathrm{Ph}$ $(83 \mu \mathrm{L}, 0.58 \mathrm{mmol})$ in THF $(2 \mathrm{~mL})$ was stirred in a sealed tube at $80^{\circ} \mathrm{C}$ for $24 \mathrm{~h}$. To the mixture were added $\mathrm{Pd}(\mathrm{dba})_{2}(57.7 \mathrm{mg}, 0.100 \mathrm{mmol})$ and $\mathrm{PMe}_{2} \mathrm{Ph}(45 \mu \mathrm{L}, 0.31 \mathrm{mmol})$. After the mixture was stirred in $80{ }^{\circ} \mathrm{C}$ for $24 \mathrm{~h}$, the solution was filtered through Celite, and the filter cake was washed with THF ( $2 \mathrm{~mL}, 3$ times). After the combined filtrate was concentrated to ca. $4 \mathrm{~mL}$, slow addition of $\mathrm{Et}_{2} \mathrm{O}(25 \mathrm{~mL})$ afforded orange crystals of 1a, which were collected by filtration, washed with $\mathrm{Et}_{2} \mathrm{O}(2 \mathrm{~mL}, 3$ times $)$ and hexane ( $\left.2 \mathrm{~mL}\right)$, and dried in vacuo to give $1 \mathrm{a} \cdot 0.3 \mathrm{C}_{4} \mathrm{H}_{8} \mathrm{O} \cdot 0.2 \mathrm{C}_{4} \mathrm{H}_{10} \mathrm{O}$ as an orange solid (85.6 mg, 0.049 mmol, 49\%). In solution, 1a was in equilibrium with 7a and $\mathrm{PMe}_{2} \mathrm{Ph}$. Spectra of $1 \mathbf{a} .{ }^{1} \mathrm{H}$ $\operatorname{NMR}\left(\mathrm{C}_{6} \mathrm{D}_{6}\right) \delta 8.22-6.37(50 \mathrm{H}, \mathrm{m}), 2.45(6 \mathrm{H}, \mathrm{s}), 2.03(12 \mathrm{H}, \mathrm{s}), 1.09$ (6H, d, J = 7.2 Hz), $1.03(12 \mathrm{H}, \mathrm{d}, J=7.6 \mathrm{~Hz}), 0.77(6 \mathrm{H}, \mathrm{d}, J=8.4 \mathrm{~Hz}) .{ }^{31} \mathrm{P}\left\{{ }^{1} \mathrm{H}\right\} \mathrm{NMR}\left(\mathrm{C}_{6} \mathrm{D}_{6}\right) \delta-4.2,-6.8$, 8.0, -10.6. Spectra of 7a. ${ }^{1} \mathrm{H}$ NMR $\left(\mathrm{C}_{6} \mathrm{D}_{6}\right) \delta 8.22-6.37(45 \mathrm{H}, \mathrm{m}), 2.37(3 \mathrm{H}, \mathrm{s}), 2.25(3 \mathrm{H}$, s), $2.244(3 \mathrm{H}, \mathrm{s}), 2.235(3 \mathrm{H}, \mathrm{s}), 2.17(3 \mathrm{H}, \mathrm{s}), 2.09(3 \mathrm{H}, \mathrm{s}), 1.38(3 \mathrm{H}, \mathrm{d}, J=11.6 \mathrm{~Hz}), 1.14$ $(3 \mathrm{H}, \mathrm{d}, J=8.0 \mathrm{~Hz}), .1 .09(3 \mathrm{H}, \mathrm{d}, J=6.4 \mathrm{~Hz}), 0.85(3 \mathrm{H}, \mathrm{d}, J=6.0 \mathrm{~Hz}), 0.75(3 \mathrm{H}, \mathrm{d}, J=$ $12.8 \mathrm{~Hz}), 0.36(3 \mathrm{H}, \mathrm{d}, J=5.6 \mathrm{~Hz}) .{ }^{31} \mathrm{P}\left\{{ }^{1} \mathrm{H}\right\} \mathrm{NMR}\left(\mathrm{C}_{6} \mathrm{D}_{6}\right) \delta 1.18,-7.4,-7.6$. Anal. Calcd. for $\mathrm{C}_{100} \mathrm{H}_{96.4} \mathrm{Cl}_{2} \mathrm{~N}_{6} \mathrm{O}_{0.5} \mathrm{P}_{4} \mathrm{Pd}_{2}\left(\mathbf{1 a} \cdot 0.3 \mathrm{C}_{4} \mathrm{H}_{8} \mathrm{O} \cdot 0.2 \mathrm{C}_{4} \mathrm{H}_{10} \mathrm{O}\right)$ : C, $66.80 \mathrm{H}, 5.40 ; \mathrm{N}, 4.67$. Found: C, $66.60 \mathrm{H}, 5.18$; N, 4.62. 


\section{Synthesis of $1 \mathrm{~b}$}
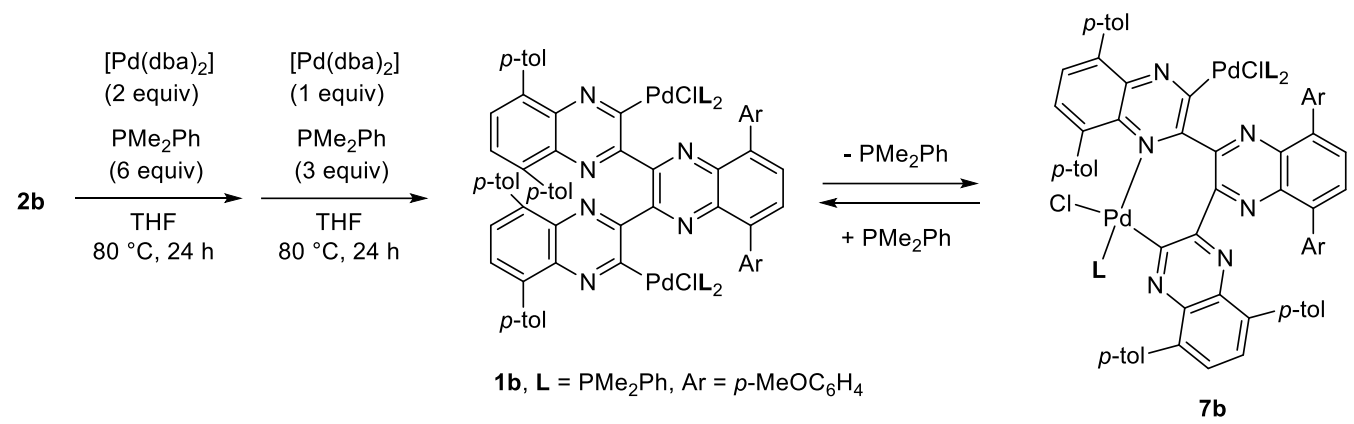

According to the typical procedure given for $\mathbf{1 a}$, the reaction was carried out using $\mathbf{2} \mathbf{b}$ (103 mg, $0.100 \mathrm{mmol}), \mathrm{Pd}(\mathrm{dba})_{2}(115 \mathrm{mg}, 0.200 \mathrm{mmol}$ and $57.4 \mathrm{mg}, 0.100 \mathrm{mmol})$, $\mathrm{PMe}_{2} \mathrm{Ph}(86 \mu \mathrm{L}, 0.60 \mathrm{mmol}$ and $43 \mu \mathrm{L}, 0.30 \mathrm{mmol})$, and THF (2.5 mL). 1b (113 mg, $0.063 \mathrm{mmol}, 63 \%$ ) was obtained as an orange solid. In solution, $\mathbf{1 b}$ was in equilibrium with 7b and $\mathrm{PMe}_{2} \mathrm{Ph}$. Spectra of $\mathbf{1 b} .{ }^{1} \mathrm{H}$ NMR $\left(\mathrm{C}_{6} \mathrm{D}_{6}\right) \delta 8.26-6.39(50 \mathrm{H}, \mathrm{m}), 3.25(6 \mathrm{H}, \mathrm{s})$, $2.45(6 \mathrm{H}, \mathrm{s}), 2.02(6 \mathrm{H}, \mathrm{s}), 1.38(3 \mathrm{H}, \mathrm{d}, J=8.0 \mathrm{~Hz}), 1.13-1.02(18 \mathrm{H}, \mathrm{m}), 0.80(6 \mathrm{H}, \mathrm{d}, J=$ $8.4 \mathrm{~Hz}) .{ }^{31} \mathrm{P}\left\{{ }^{1} \mathrm{H}\right\}$ NMR $\left(\mathrm{C}_{6} \mathrm{D}_{6}\right) \delta-4.1,-6.8,-8.1,-10.7$. Spectra of 7b. ${ }^{1} \mathrm{H}$ NMR $\left(\mathrm{C}_{6} \mathrm{D}_{6}\right)$ $\delta$ 8.26-6.39 (45H, m), $3.42(3 \mathrm{H}, \mathrm{s}), 3.27(3 \mathrm{H}, \mathrm{s}), 2.37(3 \mathrm{H}, \mathrm{s}), 2.26(3 \mathrm{H}, \mathrm{s}), 2.24(3 \mathrm{H}, \mathrm{s})$, $2.16(3 \mathrm{H}, \mathrm{s}), 1.38(3 \mathrm{H}, \mathrm{d}, J=8.0 \mathrm{~Hz}), 1.22-0.78(12, \mathrm{~m}), 0.35(3 \mathrm{H}, \mathrm{d}, J=5.6 \mathrm{~Hz}) .{ }^{31} \mathrm{P}\left\{{ }^{1} \mathrm{H}\right\}$ $\operatorname{NMR}\left(\mathrm{C}_{6} \mathrm{D}_{6}\right) \delta 1.25,-7.3$, -7.6. Anal. Calcd. for $\mathrm{C}_{98} \mathrm{H}_{92} \mathrm{Cl}_{2} \mathrm{~N}_{6} \mathrm{O}_{2} \mathrm{P}_{4} \mathrm{Pd}_{2}: \mathrm{C}, 65.63 ; \mathrm{H}, 5.17$; N, 4.69. Found: C, 65.37; H, 5.30; N, 4.62.

\section{Synthesis of $1 \mathrm{c} \cdot 0.33 \mathrm{C}_{4} \mathrm{H}_{10} \mathrm{O}$}
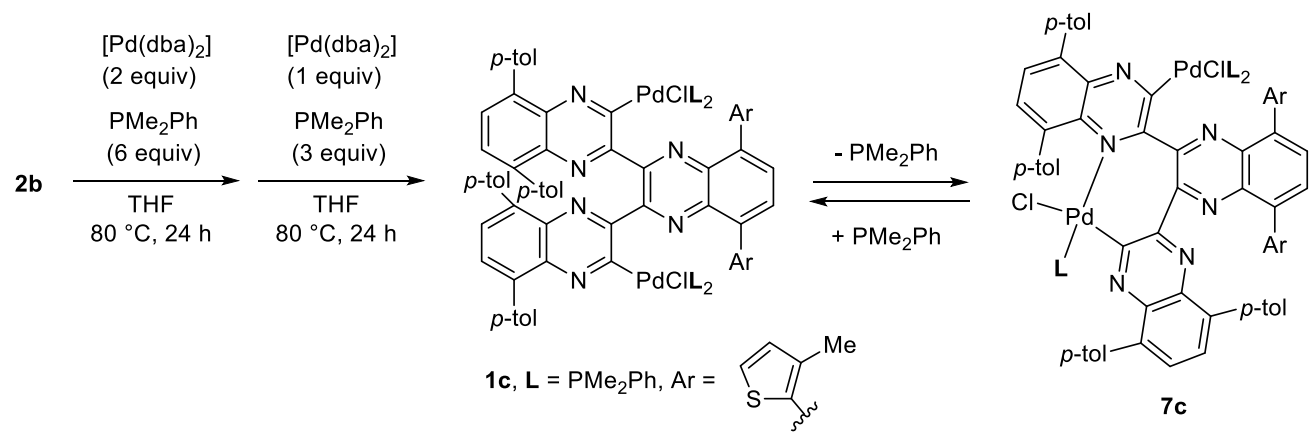

According to the typical procedure given for 1a, the reaction was carried out using $\mathbf{2 c}$ (108 mg, $0.107 \mathrm{mmol}), \mathrm{Pd}(\mathrm{dba})_{2}(124 \mathrm{mg}, 0.215 \mathrm{mmol}$ and $59.5 \mathrm{mg}, 0.103 \mathrm{mmol})$, $\mathrm{PMe}_{2} \mathrm{Ph}(90 \mu \mathrm{L}, 0.63 \mathrm{mmol}$ and $45 \mu \mathrm{L}, 0.31 \mathrm{mmol})$, and $\mathrm{THF}(2.0 \mathrm{~mL})$. 1c $0.03 \mathrm{C}_{4} \mathrm{H}_{8} \mathrm{O} \cdot 0.3 \mathrm{C}_{4} \mathrm{H}_{10} \mathrm{O}(117 \mathrm{mg}, 0.065 \mathrm{mmol}, 61 \%)$ was obtained as an orange crystalline solid. In solution, $1 \mathbf{c}$ was in equilibrium with 7c and $\mathrm{PMe}_{2} \mathrm{Ph}$. Spectra of 1c. 
${ }^{1} \mathrm{H}$ NMR $\left(\mathrm{CD}_{2} \mathrm{Cl}_{2}\right) \delta 8.05(2 \mathrm{H}, \mathrm{s}), 7.70-6.62(36 \mathrm{H}, \mathrm{m}), 6.48(4 \mathrm{H}, \mathrm{d}, J=7.6 \mathrm{~Hz}), 6.16(4 \mathrm{H}$, $\mathrm{d}, J=7.6 \mathrm{~Hz}), 2.53(6 \mathrm{H}, \mathrm{s}), 2.52(6 \mathrm{H}, \mathrm{s}), 1.85(6 \mathrm{H}, \mathrm{s}), 1.04(6 \mathrm{H}, \mathrm{d}, J=7.6 \mathrm{~Hz}), 0.91(12 \mathrm{H}$, pseudo t, $6.4 \mathrm{~Hz}), 0.83(6 \mathrm{H}, \mathrm{d}, J=7.6 \mathrm{~Hz}) .{ }^{31} \mathrm{P}\left\{{ }^{1} \mathrm{H}\right\}$ NMR $\left(\mathrm{CD}_{2} \mathrm{Cl}_{2}\right) \delta-2.1,-4.7,-5.4$, 8.0. Spectra of 7c. ${ }^{1} \mathrm{H}$ NMR $\left(\mathrm{CD}_{2} \mathrm{Cl}_{2}\right) \delta 8.07-6.21(41 \mathrm{H}, \mathrm{m}), 2.56(3 \mathrm{H}, \mathrm{s}), 2.53(3 \mathrm{H}, \mathrm{s})$, $2.44(3 \mathrm{H}, \mathrm{s}), 2.43(3 \mathrm{H}, \mathrm{s}), 2.33(3 \mathrm{H}, \mathrm{s}), 2.21(3 \mathrm{H}, \mathrm{s}), 1.27-0.86(15 \mathrm{H}, \mathrm{m}), 1.22-0.78(12 \mathrm{H}$, $\mathrm{m}),-0.05(3 \mathrm{H}, \mathrm{d}, J=5.6 \mathrm{~Hz}) .{ }^{31} \mathrm{P}\left\{{ }^{1} \mathrm{H}\right\} \mathrm{NMR}\left(\mathrm{CD}_{2} \mathrm{Cl}_{2}\right) \delta 5.5,-5.4,-5.7$. Anal. Calcd. for $\mathrm{C}_{95.33} \mathrm{H}_{91.33} \mathrm{Cl}_{2} \mathrm{~N}_{6} \mathrm{O}_{0.33} \mathrm{P}_{4} \mathrm{Pd}_{2} \mathrm{~S}_{2}\left(\mathbf{1 c} \cdot 0.33 \mathrm{C}_{4} \mathrm{H}_{10} \mathrm{O}\right): \mathrm{C}, 63.68 ; \mathrm{H}, 5.12 ; \mathrm{N}, 4$.67. Found: $\mathrm{C}$, 63.65; H, 5.18; N, 4.66.

\section{Synthesis of 6a}

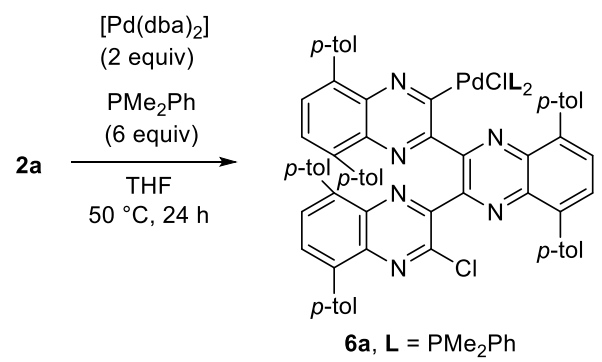

A mixture of $\mathbf{2 a}(99.3 \mathrm{mg}, 0.100 \mathrm{mmol}), \mathrm{Pd}(\mathrm{dba})_{2}(115.1 \mathrm{mg}, 0.200 \mathrm{mmol})$, and $\mathrm{PMe}_{2} \mathrm{Ph}$ $(85 \mu \mathrm{L}, 0.60 \mathrm{mmol})$ in $\mathrm{THF}(3 \mathrm{~mL})$ was stirred at $50^{\circ} \mathrm{C}$ for $24 \mathrm{~h}$. After evaporation of the solvent, the residue was purified with silica gel column chromatography (hexane:AcOEt $=6: 1$ to $2: 1)$ to give an orange solid, which was recrystallized from THF/hexane $(3 \mathrm{~mL} / 17$ $\mathrm{mL})$. The resultant crystals were collected by decantation, washed with hexane $(2 \mathrm{~mL}, 2$ times), and dried in vacuo to give $\mathbf{6 a} \cdot 0.75 \mathrm{C}_{4} \mathrm{H}_{8} \mathrm{O}$ as orange crystals $(62.4 \mathrm{mg}, 0.044 \mathrm{mmol}$, 44\%). ${ }^{1} \mathrm{H}$ NMR $\left(\mathrm{C}_{6} \mathrm{D}_{6}\right) \delta$ 7.98-6.44 (40H, m), 2.50 (3H, s), 2.41 (3H, s), 2.29 (3H, s), 2.21 $(3 \mathrm{H}, \mathrm{s}), 2.09(12 \mathrm{H}, \mathrm{s}), 1.97(3 \mathrm{H}, \mathrm{s}), 1.34$ (3H, br s), $1.13(6 \mathrm{H}$, br s), 0.81 (3H, br s). ${ }^{31} \mathrm{P}\left\{{ }^{1} \mathrm{H}\right\}$ NMR $\left(\mathrm{C}_{6} \mathrm{D}_{6}\right) \delta$-5.0, -7.6, -10.5, -13.2. Anal. Calcd. for $\mathrm{C}_{85} \mathrm{H}_{76} \mathrm{Cl}_{2} \mathrm{~N}_{6} \mathrm{O}_{0.75} \mathrm{P}_{2} \mathrm{Pd}_{2}$

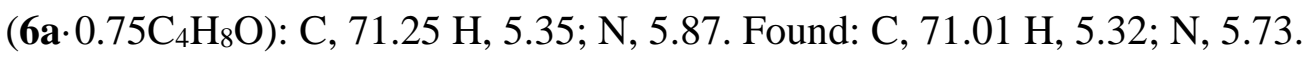




\section{Synthesis of 7a}

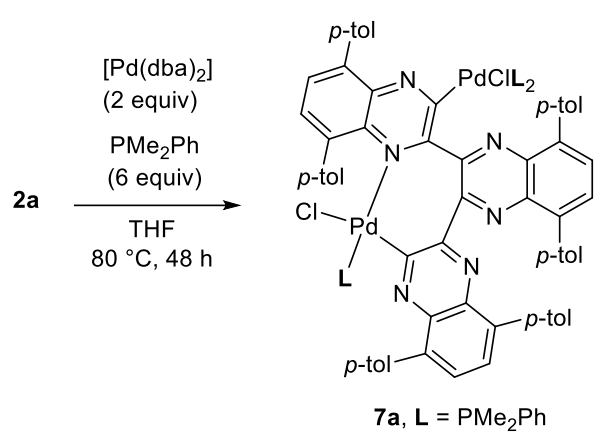

A mixture of $2 \mathbf{a}(25.1 \mathrm{mg}, 0.025 \mathrm{mmol}), \mathrm{Pd}(\mathrm{dba})_{2}(29.6 \mathrm{mg}, 0.051 \mathrm{mmol})$, and $\mathrm{PMe}_{2} \mathrm{Ph}$ $(20 \mu \mathrm{L}, 0.15 \mathrm{mmol})$ in THF $(0.5 \mathrm{~mL})$ was stirred at $80^{\circ} \mathrm{C}$ for $48 \mathrm{~h}$. After evaporation of the solvent, the residue was purified with silica gel column chromatography (hexane:AcOEt $=1: 0$ to $3: 1$ ) to give an orange solid, which was recrystallized from $\mathrm{THF} /$ hexane $(3 \mathrm{~mL} / 17 \mathrm{~mL})$. The resultant yellowish orange crystals of $7 \mathbf{a}$ were collected by decantation, washed with hexane ( $2 \mathrm{~mL}, 2$ times), and dried in vacuo to give $7 \mathbf{a}$ as yellowish orange crystals $(6.0 \mathrm{mg}, 0.003 \mathrm{mmol}, 13 \%) .{ }^{1} \mathrm{H}$ NMR $\left(\mathrm{C}_{6} \mathrm{D}_{6}\right) \delta 8.22-6.37(45 \mathrm{H}$, m), $2.37(3 \mathrm{H}, \mathrm{s}), 2.25(3 \mathrm{H}, \mathrm{s}), 2.244(3 \mathrm{H}, \mathrm{s}), 2.235(3 \mathrm{H}, \mathrm{s}), 2.17(3 \mathrm{H}, \mathrm{s}), 2.09(3 \mathrm{H}, \mathrm{s}), 1.38$ $(3 \mathrm{H}, \mathrm{d}, J=11.6 \mathrm{~Hz}), 1.14(3 \mathrm{H}, \mathrm{d}, J=8.0 \mathrm{~Hz}), 1.09(3 \mathrm{H}, \mathrm{d}, J=6.4 \mathrm{~Hz}), 0.85(3 \mathrm{H}, \mathrm{d}, J=$ $6.0 \mathrm{~Hz}), 0.75(3 \mathrm{H}, \mathrm{d}, J=12.8 \mathrm{~Hz}), 0.36(3 \mathrm{H}, \mathrm{d}, J=5.6 \mathrm{~Hz}) .{ }^{31} \mathrm{P}\left\{{ }^{1} \mathrm{H}\right\} \mathrm{NMR}\left(\mathrm{C}_{6} \mathrm{D}_{6}\right) \delta 1.18$, -7.4, -7.6. Anal. Calcd. for $\mathrm{C}_{90} \mathrm{H}_{81} \mathrm{Cl}_{2} \mathrm{~N}_{6} \mathrm{P}_{3} \mathrm{Pd}_{2}$ : C, $66.59 \mathrm{H}, 5.03 ; \mathrm{N}, 5.18$. Found: C, 66.61 $\mathrm{H}, 5.16 ; \mathrm{N}, 4.98$.

\section{Synthesis of P1a(40)}
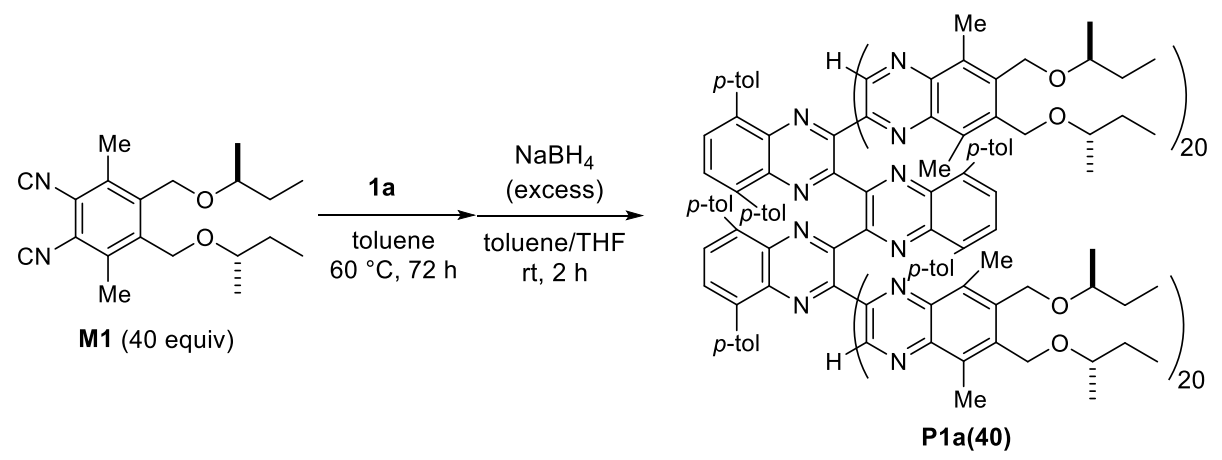

To a solution of $1 \mathrm{a} \cdot 0.3 \mathrm{C}_{4} \mathrm{H}_{8} \mathrm{O} \cdot 0.2 \mathrm{C}_{4} \mathrm{H}_{10} \mathrm{O}(3.58 \mathrm{mg}, 2.0 \mu \mathrm{mol})$ in toluene $(0.5 \mathrm{~mL})$ was added a solution of M1 $(26.26 \mathrm{mg}, 80 \mu \mathrm{mol})$ in toluene $(2.5 \mathrm{~mL})$ with vigorous stirring, and the mixture was stirred at $60{ }^{\circ} \mathrm{C}$ for $72 \mathrm{~h}$. After the complete consumption of monomer was confirmed by GPC analysis, $\mathrm{NaBH}_{4}(15.2 \mathrm{mg}, 0.40 \mathrm{mmol})$ and $\mathrm{THF}(2 \mathrm{~mL})$ were 
added to the reaction mixture and stirred for $2 \mathrm{~h}$ at room temperature. Water $(3 \mathrm{~mL})$ was added to the solution and extracted with $\mathrm{CH}_{2} \mathrm{Cl}_{2}(20 \mathrm{ml})$. The organic extract was dried over $\mathrm{Na}_{2} \mathrm{SO}_{4}$, and the solvent was evaporated. The residue was subjected to preparative GPC to give P1a(40) as a brown solid $(19.3 \mathrm{mg}, 69 \%) .{ }^{1} \mathrm{H}$ NMR $\left(\mathrm{CDCl}_{3}\right) \delta 10.0$ (terminal$H, \mathrm{~s}, 2 \mathrm{H}), 7.56$ ( $\mathrm{ArH}, 4 \mathrm{H}, \mathrm{br} \mathrm{s}), 7.34(\mathrm{ArH}, 2 \mathrm{H}, \mathrm{br} \mathrm{s}), 6.76(\mathrm{ArH}, 8 \mathrm{H}, \mathrm{br} \mathrm{s}), 6.70(\mathrm{ArH}$, $4 \mathrm{H}, \mathrm{brd}, J=7.2 \mathrm{~Hz}), 6.60(\mathrm{ArH}, 4 \mathrm{H}, \mathrm{brd}, J=7.2 \mathrm{~Hz}), 6.28(\mathrm{ArH}, \mathrm{brd}, J=7.2 \mathrm{~Hz}), 5.72$ $(\mathrm{ArH}, 4 \mathrm{H}$, br d, $J=7.2 \mathrm{~Hz}), 4.91-4.53\left(\mathrm{CH}_{2} \mathrm{O},(40 \times 4) \mathrm{H}\right.$, br m$), 3.65-3.39$ $\left(\mathrm{OCH}\left(\mathrm{CH}_{3}\right)\left(\mathrm{CH}_{2} \mathrm{CH}_{3}\right),(40 \times 2) \mathrm{H}\right.$, br m $), 3.04-2.00\left(\mathrm{ArCH}_{3},(40 \times 6+3 \times 6) \mathrm{H}\right.$, br m), 1.53-1.46 $\left(\mathrm{OCH}\left(\mathrm{CH}_{3}\right)\left(\mathrm{CH}_{2} \mathrm{CH}_{3}\right),(40 \times 4) \mathrm{H}\right.$, br m $), 1.31-1.11\left(\mathrm{OCH}\left(\mathrm{CH}_{3}\right)\left(\mathrm{CH}_{2} \mathrm{CH}_{3}\right),(40\right.$ $\times 6) \mathrm{H}$, br m), 0.98-0.64 $\left(\mathrm{OCH}\left(\mathrm{CH}_{3}\right)\left(\mathrm{CH}_{2} \mathrm{CH}_{3}\right),(40 \times 6) \mathrm{H}\right.$, br m); GPC $(\mathrm{THF}, \mathrm{g} / \mathrm{mol}): M_{\mathrm{n}}$ $=8.55 \times 10^{3}, M_{\mathrm{w}} / M_{\mathrm{n}}=1.19$.

\section{Synthesis of P1a(60)}

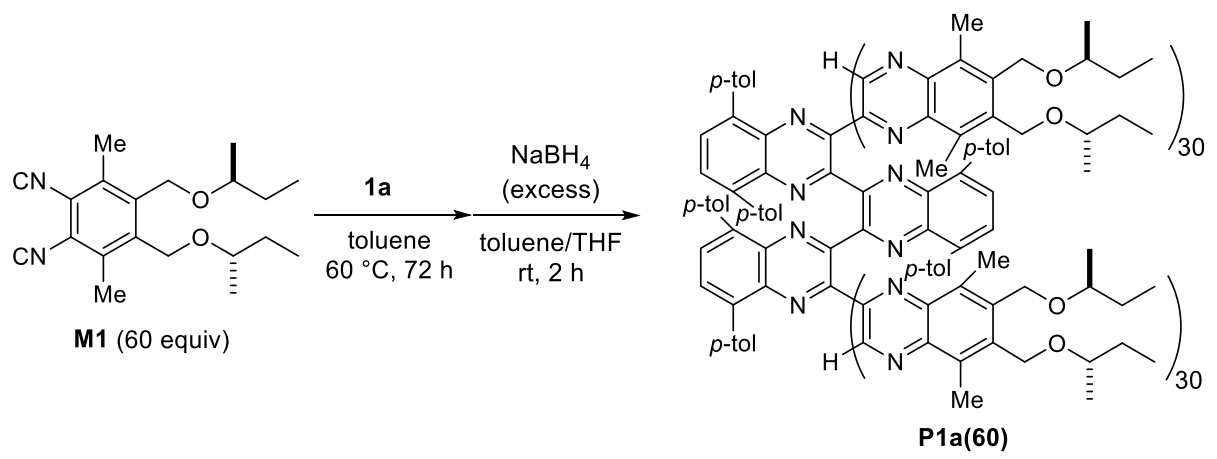

According to the typical procedure given for P1a(40), the reaction was carried out using a toluene solution $(1.0 \mathrm{~mL})$ of $\mathbf{1 a} \cdot 0.3 \mathrm{C}_{4} \mathrm{H}_{8} \mathrm{O} \cdot 0.2 \mathrm{C}_{4} \mathrm{H}_{10} \mathrm{O}(3.55 \mathrm{mg}, 2.0 \mu \mathrm{mol})$, a toluene solution (2.0 mL) of $\mathbf{M 1}(39.52 \mathrm{mg}, 120 \mu \mathrm{mol}), \mathrm{NaBH}_{4}(15.2 \mathrm{mg}, 0.40 \mathrm{mmol})$ and THF (1 mL). P1a(60) (29.7 mg, 72\%) was obtained as a brown solid. ${ }^{1} \mathrm{H}$ NMR $\left(\mathrm{CDCl}_{3}\right) \delta 10.0$ (terminal- $H, \mathrm{~s}, 2 \mathrm{H}), 7.54$ (ArH, 4H, br s), 7.32 ( $\mathrm{ArH}, 2 \mathrm{H}, \mathrm{br} \mathrm{s}), 6.75$ (ArH, 8H, br s), 6.70 (ArH, 4H, br d, J=7.6 Hz), 6.59 (ArH, 4H, br s), 6.28 (ArH, 4H, br s), $5.70(\mathrm{ArH}, 4 \mathrm{H}$, br d, $J=8.0 \mathrm{~Hz}), 4.86-4.52\left(\mathrm{CH}_{2} \mathrm{O},(60 \times 4) \mathrm{H}\right.$, br m), 3.55-3.38 $\left(\mathrm{OCH}\left(\mathrm{CH}_{3}\right)\left(\mathrm{CH}_{2} \mathrm{CH}_{3}\right)\right.$, $(60 \times 2) \mathrm{H}$, br m), 3.02-2.01 $\left(\mathrm{ArCH}_{3},(60 \times 6+3 \times 6) \mathrm{H}\right.$, br m), 1.51-1.43 $\left(\mathrm{OCH}\left(\mathrm{CH}_{3}\right)\left(\mathrm{CH}_{2} \mathrm{CH}_{3}\right),(60 \times 4) \mathrm{H}\right.$, br m $), 1.29-1.18\left(\mathrm{OCH}\left(\mathrm{CH}_{3}\right)\left(\mathrm{CH}_{2} \mathrm{CH}_{3}\right),(60 \times 6) \mathrm{H}\right.$, br m), $0.85\left(\mathrm{OCH}\left(\mathrm{CH}_{3}\right)\left(\mathrm{CH}_{2} \mathrm{CH}_{3}\right),(60 \times 6) \mathrm{H}, \mathrm{br} \mathrm{s}\right)$; GPC $(\mathrm{THF}, \mathrm{g} / \mathrm{mol}): M_{\mathrm{n}}=1.38 \times 10^{4}$, $M_{\mathrm{w}} / M_{\mathrm{n}}=1.21$. 


\section{Synthesis of P1a $(80)$}
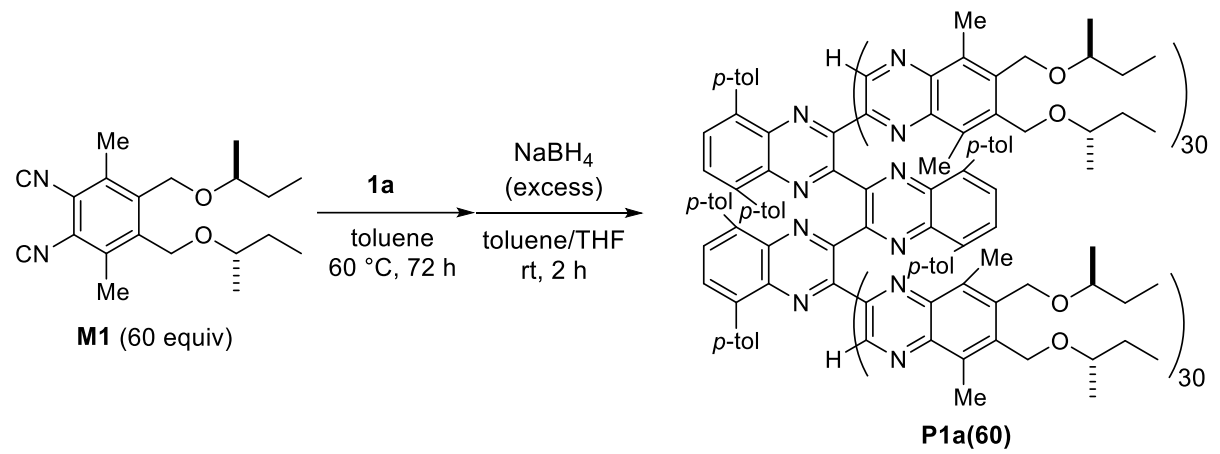

According to the typical procedure given for P1a(40), the reaction was carried out using a toluene solution $(1.0 \mathrm{~mL})$ of $4 \mathbf{a} \cdot 0.3 \mathrm{C}_{4} \mathrm{H}_{8} \mathrm{O} \cdot 0.2 \mathrm{C}_{4} \mathrm{H}_{10} \mathrm{O}(3.62 \mathrm{mg}, 2.0 \mu \mathrm{mol})$, a toluene solution $(3.0 \mathrm{~mL})$ of $\mathbf{M 1}(52.57 \mathrm{mg}, 160 \mu \mathrm{mol})$. After the complete consumption of monomer was confirmed by GPC analysis, $\mathrm{NaBH}_{4}(15.6 \mathrm{mg}, 0.41 \mathrm{mmol})$ and THF (2 $\mathrm{mL})$ were added to the reaction mixture and stirred for $4 \mathrm{~h}$ at room temperature. $\mathbf{P 1 a}(\mathbf{8 0})$ (44.0 mg, 81\%) was obtained as a brown solid. ${ }^{1} \mathrm{H}$ NMR $\left(\mathrm{CDCl}_{3}\right) \delta 10.0$ (terminal- $H$, s, 2H), $7.56(\mathrm{ArH}, 4 \mathrm{H}, \mathrm{br} \mathrm{s}), 7.35$ (ArH, 2H, br s), $6.76(\mathrm{ArH}, 8 \mathrm{H}, \mathrm{br} \mathrm{s}), 6.71(\mathrm{ArH}, 4 \mathrm{H}, \mathrm{br}$ s), $6.60(\mathrm{ArH}, 4 \mathrm{H}, \mathrm{br} \mathrm{s}), 6.29(\mathrm{ArH}, \mathrm{br} \mathrm{s}), 5.72(\mathrm{ArH}, 4 \mathrm{H}, \mathrm{br} \mathrm{s}), 4.64-4.54\left(\mathrm{CH}_{2} \mathrm{O},(80 \times\right.$ 4) $\mathrm{H}$, br m), $3.40\left(\mathrm{OCH}\left(\mathrm{CH}_{3}\right)\left(\mathrm{CH}_{2} \mathrm{CH}_{3}\right),(80 \times 2) \mathrm{H}\right.$, br s $), 3.04-2.03\left(\mathrm{ArCH}_{3},(80 \times 6+3\right.$ $\times \quad 6) \mathrm{H}, \quad$ br m), $1.55-1.44\left(\mathrm{OCH}\left(\mathrm{CH}_{3}\right)\left(\mathrm{CH}_{2} \mathrm{CH}_{3}\right),(80 \times 4) \mathrm{H}, \quad\right.$ br $\left.\mathrm{m}\right), \quad 1.21$ $\left(\mathrm{OCH}\left(\mathrm{CH}_{3}\right)\left(\mathrm{CH}_{2} \mathrm{CH}_{3}\right),(80 \times 6) \mathrm{H}\right.$, br s $), 0.87\left(\mathrm{OCH}\left(\mathrm{CH}_{3}\right)\left(\mathrm{CH}_{2} \mathrm{CH}_{3}\right),(80 \times 6) \mathrm{H}\right.$, br s $)$; GPC (THF, g/mol): $M_{\mathrm{n}}=1.72 \times 10^{4}, M_{\mathrm{w}} / M_{\mathrm{n}}=1.25$.

\section{Synthesis of P1a(100)}
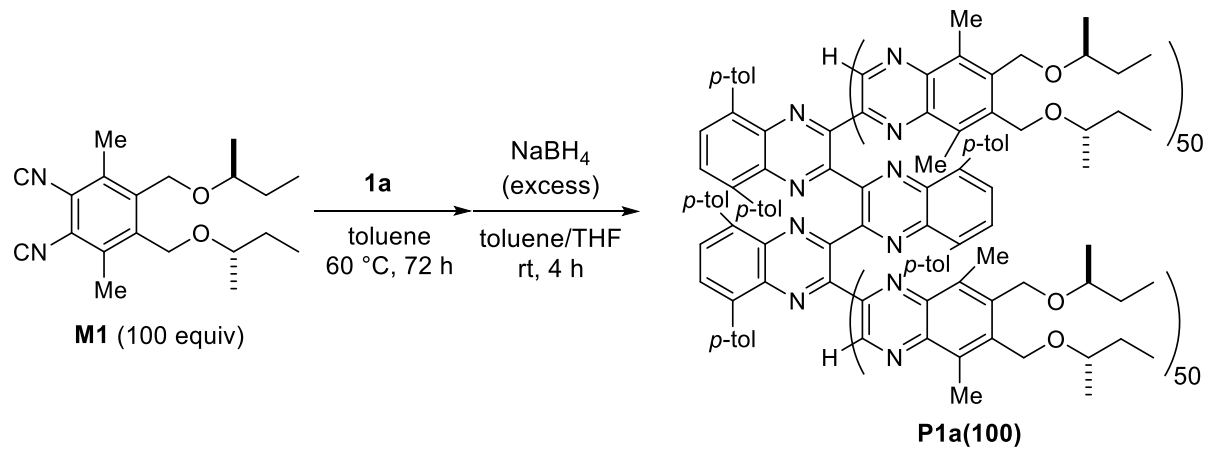

According to the typical procedure given for P1a(40), the reaction was carried out using a toluene solution $(0.5 \mathrm{~mL})$ of $1 \mathrm{a} \cdot 0.3 \mathrm{C}_{4} \mathrm{H}_{8} \mathrm{O} \cdot 0.2 \mathrm{C}_{4} \mathrm{H}_{10} \mathrm{O}(3.54 \mathrm{mg}, 2.0 \mu \mathrm{mol})$, a toluene solution $(4.5 \mathrm{~mL})$ of $\mathbf{M 1}(65.64 \mathrm{mg}, 200 \mu \mathrm{mol})$. After the complete consumption of monomer was confirmed by GPC analysis, $\mathrm{NaBH}_{4}(15.3 \mathrm{mg}, 0.40 \mathrm{mmol})$ and THF (2 
$\mathrm{mL}$ ) were added to the reaction mixture and stirred for $4 \mathrm{~h}$ at room temperature. P1a(100) (47.4 mg, 70\%) was obtained as a brown solid. ${ }^{1} \mathrm{H}$ NMR $\left(\mathrm{CDCl}_{3}\right) \delta 10.0$ (terminal- $H, \mathrm{~s}$, 2H), 7.56 ( $\mathrm{ArH}, 4 \mathrm{H}, \mathrm{br} \mathrm{s}), 7.35$ ( $\mathrm{ArH}, 2 \mathrm{H}, \mathrm{br} \mathrm{s}), 6.77$ ( $\mathrm{ArH}, 8 \mathrm{H}, \mathrm{br} \mathrm{s}), 6.71(\mathrm{ArH}, 4 \mathrm{H}, \mathrm{br}$ s), $6.61(\mathrm{ArH}, 4 \mathrm{H}, \mathrm{br} \mathrm{ds}), 6.28(\mathrm{ArH}, \mathrm{br} \mathrm{s}), 5.72(\mathrm{ArH}, 4 \mathrm{H}, \mathrm{br} \mathrm{s}), 4.64-4.54\left(\mathrm{CH}_{2} \mathrm{O},(100\right.$ $\times 4) \mathrm{H}$, br m), $3.40\left(\mathrm{OCH}\left(\mathrm{CH}_{3}\right)\left(\mathrm{CH}_{2} \mathrm{CH}_{3}\right),(100 \times 2) \mathrm{H}\right.$, br s), 3.04-2.03 $\left(\mathrm{ArCH}_{3},(100 \times 6\right.$ $+3 \times 6) \mathrm{H}$, br m), 1.55-1.45 $\left(\mathrm{OCH}\left(\mathrm{CH}_{3}\right)\left(\mathrm{CH}_{2} \mathrm{CH}_{3}\right),(100 \times 4) \mathrm{H}\right.$, br m), 1.21 $\left(\mathrm{OCH}\left(\mathrm{CH}_{3}\right)\left(\mathrm{CH}_{2} \mathrm{CH}_{3}\right),(100 \times 6) \mathrm{H}\right.$, br s $), 0.88\left(\mathrm{OCH}\left(\mathrm{CH}_{3}\right)\left(\mathrm{CH}_{2} \mathrm{CH}_{3}\right),(100 \times 6) \mathrm{H}\right.$, br s $)$; GPC $(\mathrm{THF}, \mathrm{g} / \mathrm{mol}): M_{\mathrm{n}}=2.18 \times 10^{4}, M_{\mathrm{w}} / M_{\mathrm{n}}=1.26$.

\section{Synthesis of P1b(60)}
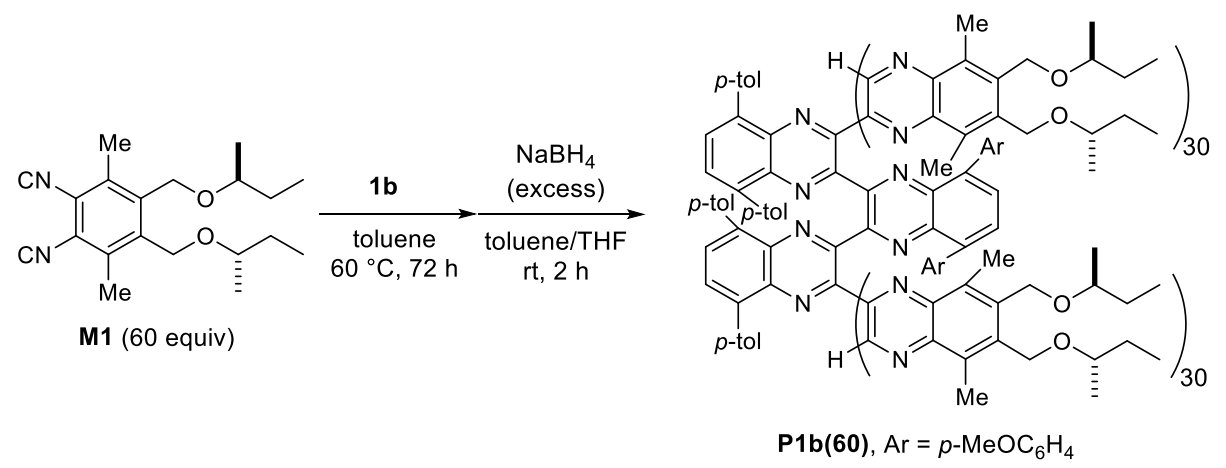

According to the typical procedure given for P1a(40), the reaction was carried out using a toluene solution $(2.0 \mathrm{~mL})$ of $\mathbf{1 b}(3.70 \mathrm{mg}, 2.0 \mu \mathrm{mol})$, a toluene solution $(1.0 \mathrm{~mL})$ of $\mathbf{M 1}$ (39.47 mg, $120 \mu \mathrm{mol}), \mathrm{NaBH}_{4}(15.1 \mathrm{mg}, 0.40 \mathrm{mmol}$ ) and THF (2 mL). P1b(60) (30.4 mg, $73 \%)$ was obtained as a brown solid. ${ }^{1} \mathrm{H}$ NMR $\left(\mathrm{CDCl}_{3}\right) \delta 10.0$ (terminal- $\left.H, \mathrm{~s}, 2 \mathrm{H}\right), 7.54$ (ArH, 4H, br s), 7.39 (ArH, 2H, br s), 7.08 (ArH, 4H, br d, J=7.2 Hz), 6.78-6.62 (ArH, $12 \mathrm{H}, \mathrm{m}), 6.25(\mathrm{ArH}, 4 \mathrm{H}, \mathrm{br} \mathrm{d}, J=8.0 \mathrm{~Hz}), 5.62(\mathrm{ArH}, 4 \mathrm{H}, \mathrm{br} \mathrm{d}, J=8.0 \mathrm{~Hz}), 4.64-4.53$ $\left(\mathrm{CH}_{2} \mathrm{O}, 60 \times 4\right) \mathrm{H}$, br m), 3.56-3.40 $\left(\mathrm{OCH}\left(\mathrm{CH}_{3}\right)\left(\mathrm{CH}_{2} \mathrm{CH}_{3}\right)+\mathrm{OCH}_{3},(60 \times 2+6) \mathrm{H}\right.$, br m), 3.04-1.94 $\left(\mathrm{ArCH}_{3},(60 \times 6+2 \times 6) \mathrm{H}\right.$, br m $), 1.56-1.44\left(\mathrm{OCH}\left(\mathrm{CH}_{3}\right)\left(\mathrm{CH}_{2} \mathrm{CH}_{3}\right),(60 \times 4) \mathrm{H}\right.$, br m), $\quad 1.31-1.13 \quad\left(\mathrm{OCH}\left(\mathrm{CH}_{3}\right)\left(\mathrm{CH}_{2} \mathrm{CH}_{3}\right), \quad(60 \times \quad 6) \mathrm{H}, \quad\right.$ br $\left.\quad \mathrm{m}\right), \quad 0.98-0.67$ $\left(\mathrm{OCH}\left(\mathrm{CH}_{3}\right)\left(\mathrm{CH}_{2} \mathrm{CH}_{3}\right),(60 \times 6) \mathrm{H}\right.$, br m); GPC $(\mathrm{THF}, \mathrm{g} / \mathrm{mol}): M_{\mathrm{n}}=1.20 \times 10^{4}, M_{\mathrm{w}} / M_{\mathrm{n}}=$ 1.19 . 


\section{Synthesis of P1c(60)}
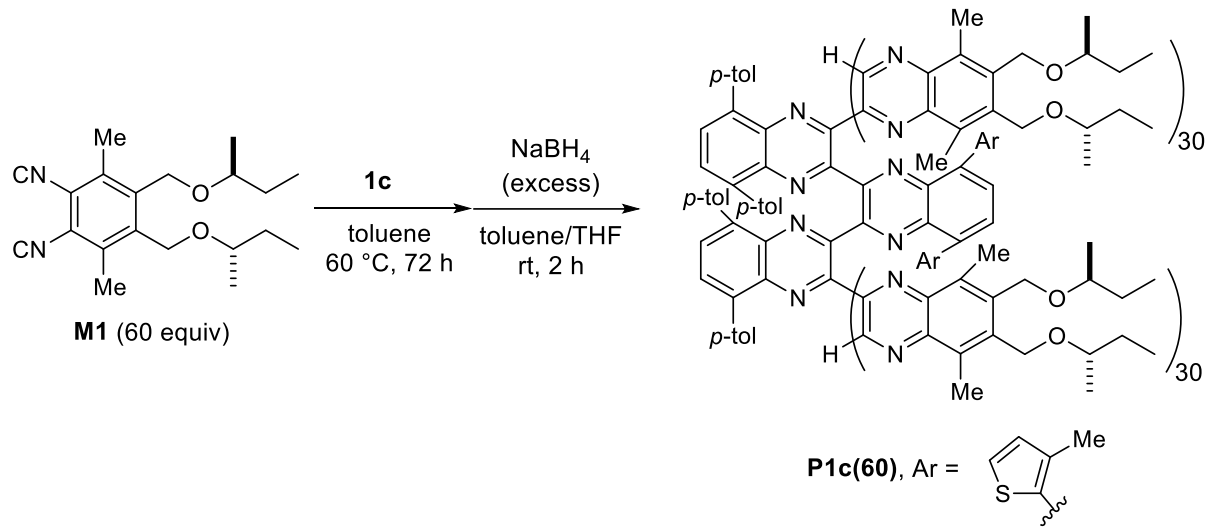

According to the typical procedure given for P1a(40), the reaction was carried out using a toluene solution $(1.0 \mathrm{~mL})$ of $1 \mathbf{c} \cdot 0.33 \mathrm{C}_{4} \mathrm{H}_{10} \mathrm{O}(3.63 \mathrm{mg}, 2.0 \mu \mathrm{mol})$, a toluene solution $(2.0 \mathrm{~mL})$ of $\mathbf{M 1}(39.26 \mathrm{mg}, 120 \mu \mathrm{mol}), \mathrm{NaBH}_{4}(15.8 \mathrm{mg}, 0.42 \mathrm{mmol})$ and THF $(2 \mathrm{~mL})$. P1c(60) (26.8 mg, 65\%) was obtained as a brown solid. ${ }^{1} \mathrm{H}$ NMR $\left(\mathrm{CDCl}_{3}\right) \delta 10.0$ (terminal- $H, 2 \mathrm{H}$, br s), $7.58(\mathrm{ArH}, 4 \mathrm{H}$, br s), $7.40(\mathrm{ArH}, 2 \mathrm{H}, \mathrm{br} \mathrm{s}), 6.78(\mathrm{ArH}, 8 \mathrm{H}, \mathrm{br} \mathrm{s})$, $6.51(\mathrm{ArH}, 4 \mathrm{H}, \mathrm{br} \mathrm{s}), 6.29(\mathrm{ArH}, 4 \mathrm{H}, \mathrm{br} \mathrm{s}), 5.75(\mathrm{ArH}, 2 \mathrm{H}, \mathrm{br} \mathrm{d}, J=5.2 \mathrm{~Hz}), 5.43(\mathrm{ArH}$, $2 \mathrm{H}$, br d, $J=5.2 \mathrm{~Hz}), 4.63-4.53\left(\mathrm{CH}_{2} \mathrm{O},(60 \times 4) \mathrm{H}\right.$, br m $), 3.54-3.40\left(\mathrm{OCH}\left(\mathrm{CH}_{3}\right)\left(\mathrm{CH}_{2} \mathrm{CH}_{3}\right)\right.$, $(60 \times 2) \mathrm{H}$, br s), 3.04-2.03 $\left(\mathrm{ArCH}_{3},(40 \times 6+3 \times 6) \mathrm{H}\right.$, br m), 1.75-1.45 $\left(\mathrm{OCH}\left(\mathrm{CH}_{3}\right)\left(\mathrm{CH}_{2} \mathrm{CH}_{3}\right),(60 \times 4) \mathrm{H}\right.$, br m $), 1.31-1.09\left(\mathrm{OCH}\left(\mathrm{CH}_{3}\right)\left(\mathrm{CH}_{2} \mathrm{CH}_{3}\right),(60 \times 6) \mathrm{H}\right.$, br

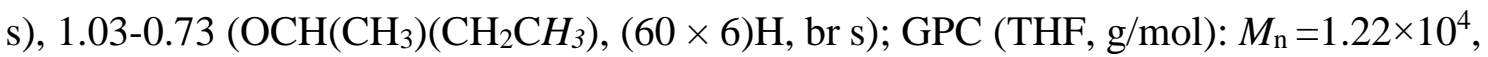
$M_{\mathrm{W}} / M_{\mathrm{n}}=1.24$.

\section{Synthesis of P2}

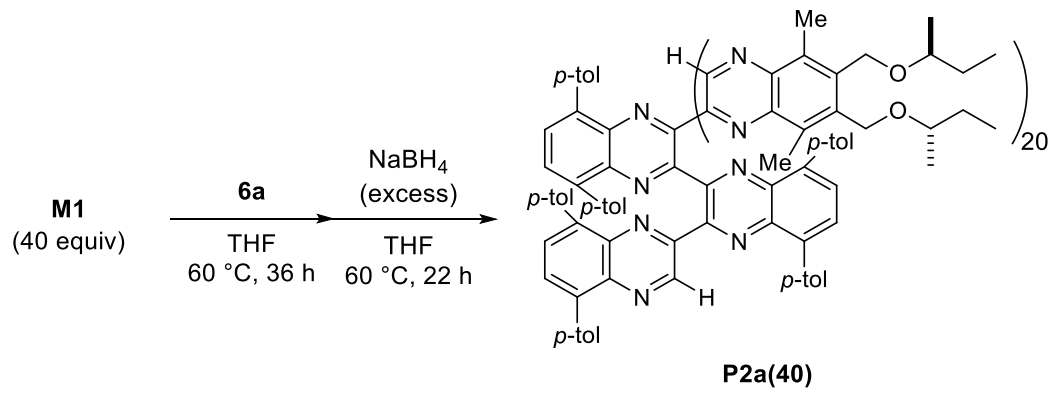

To a solution of $6 \mathbf{a} \cdot 0.75 \mathrm{C}_{4} \mathrm{H}_{8} \mathrm{O}(2.87 \mathrm{mg}, 2.0 \mu \mathrm{mol})$ in THF $(1.0 \mathrm{~mL})$ was added a solution of M1 $(26.33 \mathrm{mg}, 80 \mu \mathrm{mol})$ in THF $(2.0 \mathrm{~mL})$ with vigorous stirring, and the mixture was stirred at $60{ }^{\circ} \mathrm{C}$ for $38 \mathrm{~h}$. After the complete consumption of monomer was confirmed by GPC analysis, $\mathrm{NaBH}_{4}(15.3 \mathrm{mg}, 0.40 \mathrm{mmol})$ was added to the reaction mixture and stirred for $22 \mathrm{~h}$ at $50{ }^{\circ} \mathrm{C}$. Water $(3 \mathrm{~mL})$ was added to the solution and extracted with $\mathrm{CH}_{2} \mathrm{Cl}_{2}(20$ 
$\mathrm{ml}$ ). The organic extract was dried over $\mathrm{Na}_{2} \mathrm{SO}_{4}$, and the solvent was evaporated. The residue was subjected to preparative GPC to give $\mathbf{P 4}$ as a brown solid $(22.8 \mathrm{mg}, 81 \%) .{ }^{1} \mathrm{H}$ NMR $\left(\mathrm{CDCl}_{3}\right) \delta$ 8.01-5.99 $\left(\mathrm{ArH}, 30 \mathrm{H}\right.$, br m), 4.63-4.54 $\left(\mathrm{CH}_{2} \mathrm{O},(40 \times 4) \mathrm{H}\right.$, br m), 3.40 $\left(\mathrm{OCH}\left(\mathrm{CH}_{3}\right)\left(\mathrm{CH}_{2} \mathrm{CH}_{3}\right),(40 \times 2) \mathrm{H}\right.$, br s $), 3.04-2.03\left(\mathrm{ArCH}_{3},(40 \times 6+3 \times 6) \mathrm{H}\right.$, br m), 1.53-1.44 $\left(\mathrm{OCH}\left(\mathrm{CH}_{3}\right)\left(\mathrm{CH}_{2} \mathrm{CH}_{3}\right),(40 \times 2) \mathrm{H}\right.$, br m), $1.21\left(\mathrm{OCH}\left(\mathrm{CH}_{3}\right)\left(\mathrm{CH}_{2} \mathrm{CH}_{3}\right),(40 \times\right.$ $6) \mathrm{H}$, br s), $0.87\left(\mathrm{OCH}\left(\mathrm{CH}_{3}\right)\left(\mathrm{CH}_{2} \mathrm{CH}_{3}\right),(40 \times 6) \mathrm{H}\right.$, br s), peaks derived from end groups were observed at 10.03 and $9.55 \mathrm{ppm}$ with a ratio of 1:0.87; GPC (THF, g/mol): $M_{\mathrm{n}}=$ $9.05 \times 10^{3}, M_{\mathrm{w}} / M_{\mathrm{n}}=1.21$. 


\section{Characterization of 1 in solution}
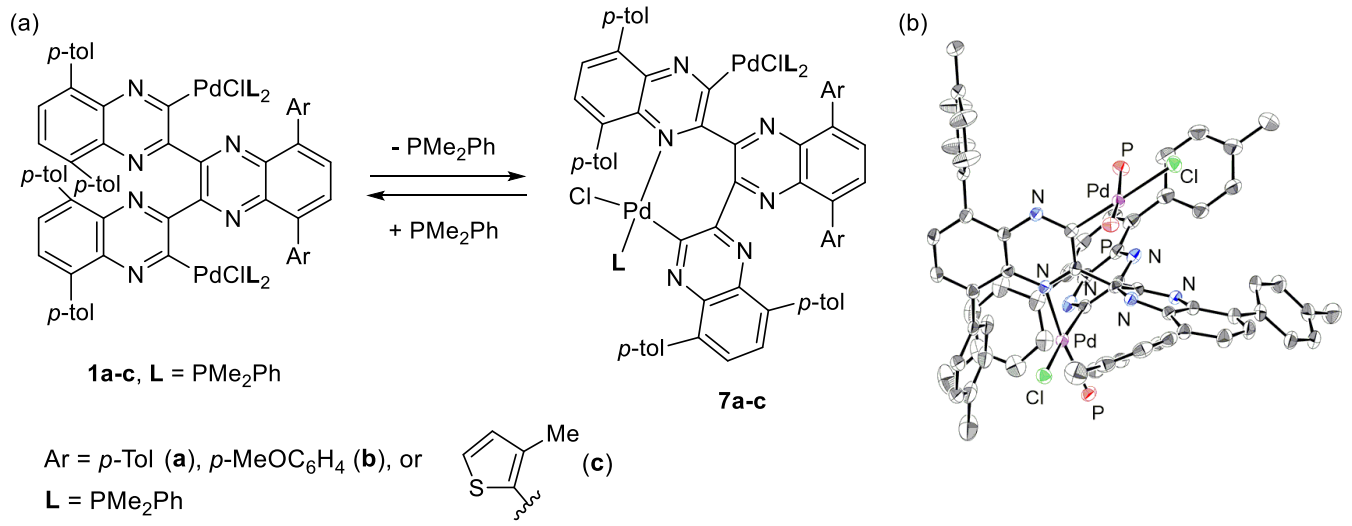

Figure S1. (a) Equilibrium between 1 and 7. (b) ORTEP drawing of 7a. Substituents on phosphorous atoms, hydrogen atoms, and solvated molecules are omitted for clarity.

When $1 \mathbf{a}-\mathbf{c}$ were dissolved in $\mathrm{C}_{6} \mathrm{D}_{6}$, we observed the formation of a new palladium complex together with $1 \mathbf{1}-\mathbf{c}$ and the free $\mathrm{PMe}_{2} \mathrm{Ph}$ ligand. The new species was identified as triphosphine-dipalladium complex 7, which was synthesized independently and unambiguously characterized by X-ray analysis of $7 \mathbf{a}$ (Figure S1). One of four $\mathrm{PMe}_{2} \mathrm{Ph}$ in $1 \mathbf{a}$ dissociated from a palladium atom to afford complex $\mathbf{7 a}$, in which the palladium center was coordinated by $\mathrm{N}$ atom of the quinoxaline. When $\mathrm{PMe}_{2} \mathrm{Ph}$ was added to the solution of $7 \mathbf{a}$, a rapid formation of a mixture of $\mathbf{1 a}$ and $7 \mathbf{a}$ was observed. These results indicate that $1 \mathbf{a}-\mathbf{c}$ are in equilibrium with $7 \mathbf{a}-\mathbf{c}$ and free $\mathrm{PMe}_{2} \mathrm{Ph}$ in the solution state.

Polymerization of M1 was investigated using the dipalladium complex 7a (Table S1). Although 7a exhibited higher polymerization activity than 1a, the resultant polymer had a slightly larger polydispersity index (PDI) value of 1.27 (entry 2). Addition of 1 equiv of $\mathrm{PMe}_{2} \mathrm{Ph}$ to the polymerization of $\mathbf{M 1}$ by using $7 \mathbf{a}$ improved the value of the PDI to 1.21 (entry 3), which indicate that the 1:1 ratio of $\mathrm{Pd}$ and $\mathrm{PMe}_{2} \mathrm{Ph}$ is important to obtain the PQX with a narrow PDI. 
Table S1. Polymerization of M1 Using 1a or 7a as Initiator

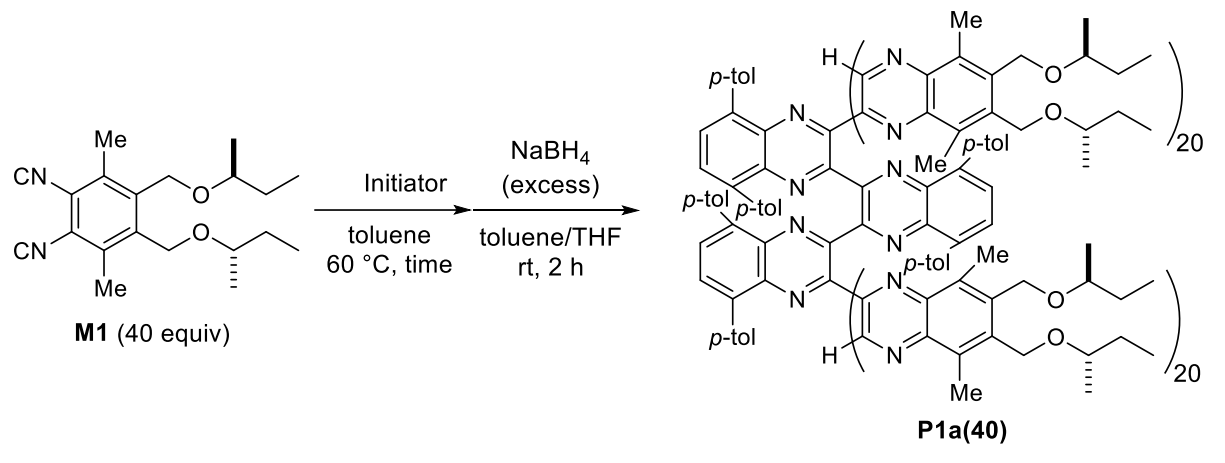

\begin{tabular}{|l|l|l|l|l|l|}
\hline Entry & Initiator & Time (h) & Yield (\%) & $M_{\mathrm{n}}\left(/ 10^{4}\right)^{a}$ & $M_{\mathrm{w}}{ }^{\prime} M_{\mathrm{n}}{ }^{a}$ \\
\hline 1 & $\mathbf{1 a}$ & 72 & 69 & 0.86 & 1.19 \\
\hline 2 & $\mathbf{7 a}$ & 42 & 80 & 0.93 & 1.27 \\
\hline 3 & $\mathbf{7 a}^{b}$ & 72 & 80 & 0.87 & 1.21 \\
\hline
\end{tabular}

${ }^{a}$ Molecular weights were determined by GPC with polystyrene standards. ${ }^{b}$ Polymerization was carried out in the presence of 1 equiv of $\mathrm{PMe}_{2} \mathrm{Ph}$. 
4 MALDI-TOF-MS and ${ }^{1}$ H NMR Analysis of the Polymers

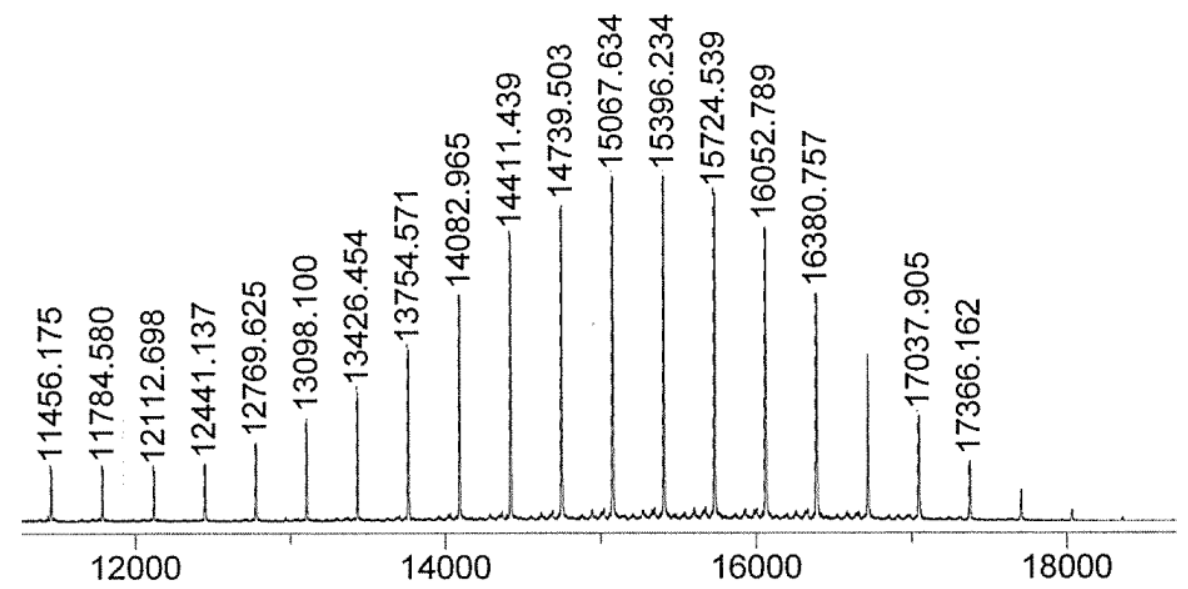

Figure S2. MALDI-TOF-MS (DCTB-Na) spectrum of P1a(40). 
(a) $\mathrm{P} 1 \mathrm{a}(\mathbf{4 0})$

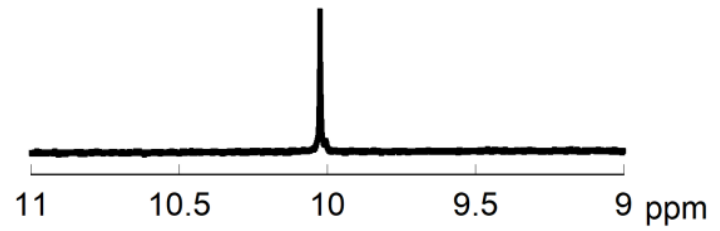

(c) P4

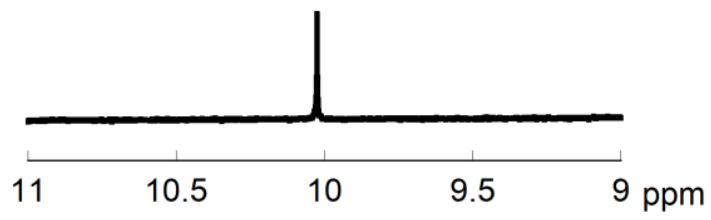

(b) $\mathrm{P} 2 \mathrm{a}(\mathbf{4 0})$

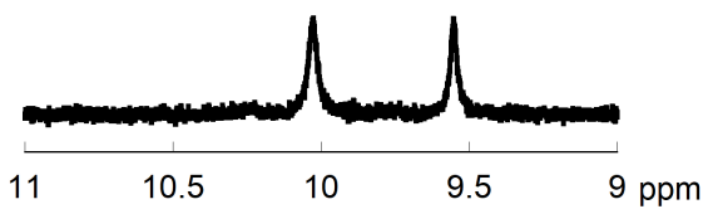

(d) P5

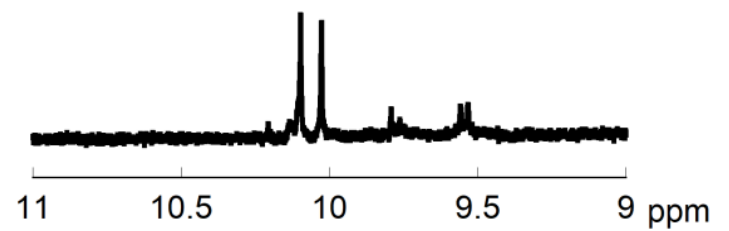

P4

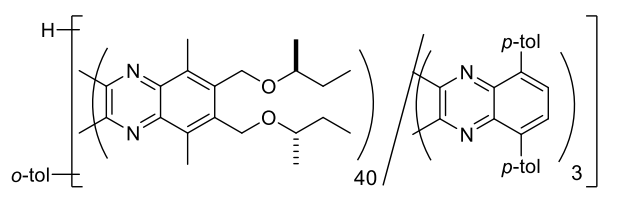

P5

Figure S3. ${ }^{1} \mathrm{H}$ NMR spectra $\left(\mathrm{CDCl}_{3}\right)$ from 11.0 to $9.0 \mathrm{ppm}$ of (a) P1a(40), (b) P2a(40), (c) P4, and (d) P5.

The structures of the polymers were characterized by a ${ }^{1} \mathrm{H}$ NMR spectroscopy (Figure $\mathrm{S} 1)$. P1a(40) exhibited only a single signal of the terminal $\mathrm{H}$ atom at $10.0 \mathrm{ppm}$, which was derived from the chiral units (Figure S1a). In contrast, P2a(40) showed two distinctive protons that are assignable to that on the terquinoxaline unit along with the chiral unit (Figure S1b). We considered that the chloride atom on the terquinoxaline was partially reduced by the treatment of $\mathrm{NaBH}_{4}$ during the synthesis of P2a(40). As a comparison, we synthesized homo-43mer of M1 $\left(\mathbf{P 4}, M_{\mathrm{n}}=9.42 \times 10^{3}, M_{\mathrm{w}} / M_{\mathrm{n}}=1.13\right)$ and a random co-43mer $\left(\mathbf{P 5} M_{\mathrm{n}}=1.02 \times 10^{4}, M_{\mathrm{w}} / M_{\mathrm{n}}=1.18\right)$, which consists of 40 chiral units along with 5 tolyl-containing quinoxaline units on average, according to the reported procedures. ${ }^{1,3}$ The ${ }^{1} \mathrm{H}$ NMR spectrum of $\mathbf{P 4}$ showed only terminal protons derived from the chiral unit. (Figure 3b). On the other hand, P5 exhibited several terminal-H peaks derived from the chiral unit and the tolyl unit in the range of 10.1-9.5 ppm (Figure 3c). We consider that the presence of the tolyl unit near the terminal chiral unit resulted in an appearance of multiple signals around $10.0 \mathrm{ppm}$. These results suggest that chain growth occurred efficiently on both sides of divalent initiator 1a. 


\section{Chiroptical Properties of the Polymers}

\section{Measurement of P1a-c(n) and P2a(40).}

Typical procedure for $\mathrm{CD}$ Measurement in $\mathrm{CHCl}_{3}$ : $M$-helical conformation. Purified sample of P1a(40) (2.60 mg) was dissolved into chloroform (10 mL) at rt. A $1 \mathrm{~mL}$ portion of the solution was transferred into another volumetric flask by a measuring pipette. The final volume of the solution was adjusted to $10 \mathrm{~mL}$ with chloroform. The solution was subjected to the measurements of CD intensity at $20^{\circ} \mathrm{C}$. Then, the solution was heated at $50{ }^{\circ} \mathrm{C}$ for $18 \mathrm{~h}$. After being cooled, the solution was subjected to the measurements of CD intensity at $20{ }^{\circ} \mathrm{C}$. We confirmed that the both $\mathrm{CD}$ spectra were identical and that the purified polymer adopted $M$-helical conformation.

Typical procedure for $C D$ measurement in 1,1,2-Trichloroethane: $P$-helical conformation. A solution of P1a(40) (2.60 mg) ( $M$-helical conformation) in chloroform $(10 \mathrm{~mL})$ was prepared. A $1 \mathrm{~mL}$ portion of the solution was transferred into another volumetric flask by a measuring pipette. The solvent was evaporated under vacuum. The residue was dissolved by 1,1,2-trichloroethane of $10 \mathrm{~mL}$. The solution was transferred into J-Young Schlenk and heated at $80{ }^{\circ} \mathrm{C}$ for $72 \mathrm{~h}$. After being cooled, the solution was subjected to the measurements of $\mathrm{CD}$ intensity at $20{ }^{\circ} \mathrm{C}$. 
Table S2. $g_{\text {abs }}$ values of polymers in $\mathrm{CHCl}_{3}$ or $1,1,2-\mathrm{TCE}^{\mathrm{a}}$

\begin{tabular}{|l|l|l|}
\hline & $\mathrm{CHCl}_{3}\left(/ 10^{-3}\right)$ & $1,1,2-\mathrm{TCE}\left(/ 10^{-3}\right)$ \\
\hline $\mathbf{P 1 a}(\mathbf{4 0})$ & -1.98 & +1.71 \\
\hline $\mathbf{P 1 a ( 6 0 )}$ & -2.38 & +2.85 \\
\hline P1a(80) & -2.34 & +2.81 \\
\hline P1a(100) & -2.40 & $+2.90^{b}$ \\
\hline P1b(60) & -2.21 & +2.59 \\
\hline P1c(60) & -2.17 & +2.73 \\
\hline P2a(40) & -2.07 & +1.94 \\
\hline
\end{tabular}

${ }^{a} g_{\text {abs }}$ values were measured at $366.0 \mathrm{~nm} .{ }^{b} \mathrm{~A}$ mixed $1,1,2-\mathrm{TCE} / \mathrm{THF}(8 / 2, \mathrm{v} / \mathrm{v})$ solvent was used for P1a(100) due to its low solubility in pure 1,1,2-TCE.

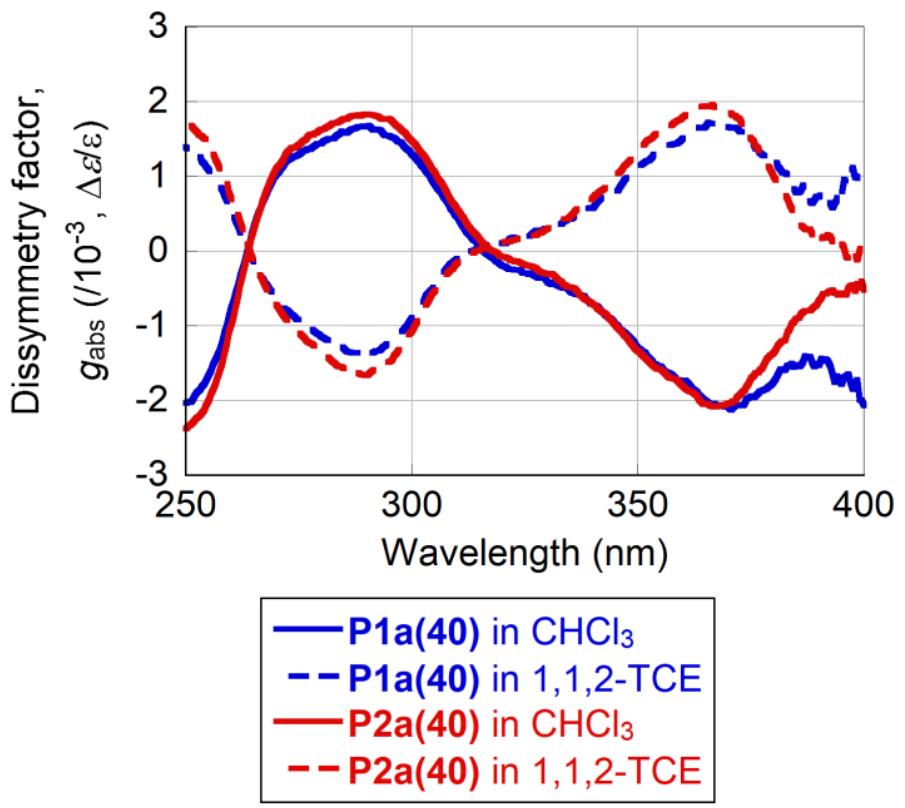

Figure S4. CD spectra of $\mathbf{P 1 a ( 4 0 )}$ and $\mathbf{P 2 a}(40)$ in $\mathrm{CHCl}_{3}$ or $1,1,2-\mathrm{TCE}$ $\left(2.60-2.99 \times 10^{-2} \mathrm{~g} / \mathrm{L}\right)$. 


\section{Fluorescent Properties of Terquinoxalines 2}

Table S3. PL properties of 2 in $\mathrm{CHCl}_{3}$ or $1,1,2-\mathrm{TCE}$

\begin{tabular}{|c|c|c|c|c|}
\hline & \multicolumn{2}{|c|}{$\mathrm{CHCl}_{3}$} & \multicolumn{2}{c|}{$1,1,2-\mathrm{TCE}$} \\
\hline & $\lambda_{\mathrm{PL}}(\mathrm{nm})^{a}$ & $\Phi_{\mathrm{PL}}(\%)^{b}$ & $\lambda_{\mathrm{PL}}(\mathrm{nm})^{a}$ & $\Phi_{\mathrm{PL}}(\%)^{b}$ \\
\hline 2a & 512.5 & 20.5 & 516.0 & 19.6 \\
\hline 2b & 565.5 & 44.1 & 568.5 & 42.0 \\
\hline 2c & 597.5 & 57.4 & 613.5 & 47.2 \\
\hline
\end{tabular}

${ }^{a}$ Maximum PL wavelength. ${ }^{b}$ Absolute photoluminescent quantum yield. 


\section{Circularly Polarized Luminescence (CPL) Measurement}

The CPL spectra were recorded at room temperature on a JASCO CPL-200 with an SQgrade quartz cuvette (a path length of $10 \mathrm{~mm}$ ). A scanning rate of $50 \mathrm{~nm} / \mathrm{min}$, an excitation bandwidth of $3000 \mu \mathrm{m}$, a monitoring bandwidth of $3000 \mu \mathrm{m}$, a response time of 8 seconds, and 5 times accumulation were employed. The CPL dissymmetry factor $g_{\text {lum }}$ is defined as $g_{\text {lum }}=2\left(\mathrm{I}_{\mathrm{L}}-\mathrm{I}_{\mathrm{R}}\right) /\left(\mathrm{I}_{\mathrm{L}}+\mathrm{I}_{\mathrm{R}}\right)$, where $\mathrm{I}_{\mathrm{L}}$ and $\mathrm{I}_{\mathrm{R}}$ are the fluorescence intensities of the right- and left-handed circularly polarized light, respectively. The value of $g_{\text {lum }}$ can be calculated on a JASCO CPL-200 as follows.

$$
\begin{aligned}
g_{\text {lum }} & =[\text { PL ellipticity }(\text { mdeg })] /[\text { PL intensity }(\mathrm{V})] /(1000 \times 180 / 4 \pi) \\
& =[\text { PL ellipticity }(\text { mdeg })] /[\text { PL intensity }(\mathrm{V})] \times\left(6.98 \times 10^{-5}\right)
\end{aligned}
$$

The validity of the coefficient $\left(6.98 \times 10^{-5}\right)$ was also confirmed by the measurement of previously reported CPL materials ${ }^{5}$ (europium(III) ions coordinated by chiral N,N'-bis(1phenylethyl)-2,6-pyridinedicarboxamide in $\mathrm{MeCN}$ ). For a curve fitting of observed CPL spectra, following equation was adopted on the basis of the supposition that the CPL signals are expressed as a Gaussian distribution functions of the wavenumber.

$$
\mathrm{I}_{\mathrm{L}}-\mathrm{I}_{\mathrm{R}}=\mathrm{a} \times \exp \left(\mathrm{b} \times(1 / \lambda-\mathrm{c})^{2}\right)
$$

where $\lambda$ is the wavelength (i.e. $1 / \lambda$ is the wavenumber), $a, b$, and $c$ are variables for the curve fitting. Nonlinear least-squares fitting of $I_{L}-I_{R}$ versus $\lambda$ was performed by using the Solver Function in Microsoft Office Excel 2013. Sums of the squares of the deviation were minimized by varying 3 parameters $(a, b$, and $c)$. 
Table S4. PL and CPL Properties of polymers in $\mathrm{CHCl}_{3}$ or 1,1,2-TCE

\begin{tabular}{lllll}
\hline & & $\lambda_{\mathrm{PL}}(\mathrm{nm})^{a}$ & $g_{\text {lum }}\left(/ 10^{-3}\right)^{b}$ & $\Phi_{\mathrm{PL}}(\%)^{c}$ \\
\hline $\mathrm{CHCl}_{3}$ & $\mathbf{P 1 a ( 6 0 )}$ & $(666), 476$ & -1.40 & 2.9 \\
& P1b(60) & 515 & -1.50 & 18.9 \\
& P1c(60) & 570 & -1.19 & 17.8 \\
& P2a(40) & 501 & 0.36 & 5.0 \\
\hline $1,1,2-$ & P1a(60) & $(664), 477$ & 1.13 & 3.2 \\
$\mathrm{TCE}$ & $\mathbf{P 1 b ( 6 0 )}$ & 521 & 1.26 & 19.7 \\
& P1c(60) & 569 & 0.94 & 18.2 \\
& P2a(40) & 501 & 0.59 & 5.4 \\
\hline
\end{tabular}

${ }^{a}$ Maximum PL wavelength. ${ }^{b} g_{\text {lum }}$ was determined at maximum PL wavelength. ${ }^{c}$ Absolute photoluminescent quantum yield. 

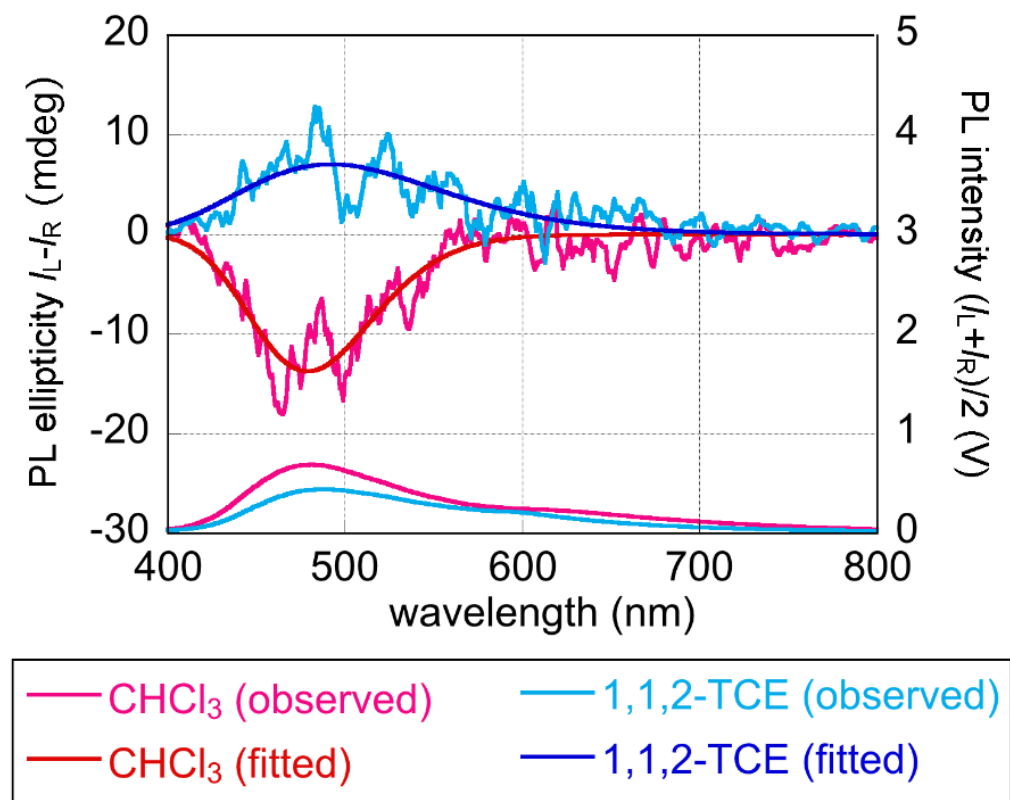

Figure S5. CPL and PL spectra of P1a(60) $\left(2.86 \times 10^{-3} \mathrm{~g} / \mathrm{L}\right)$ in $\mathrm{CHCl}_{3}$ and $1,1,2-\mathrm{TCE}$ $(\lambda \mathrm{ex}=290.0 \mathrm{~nm})$.

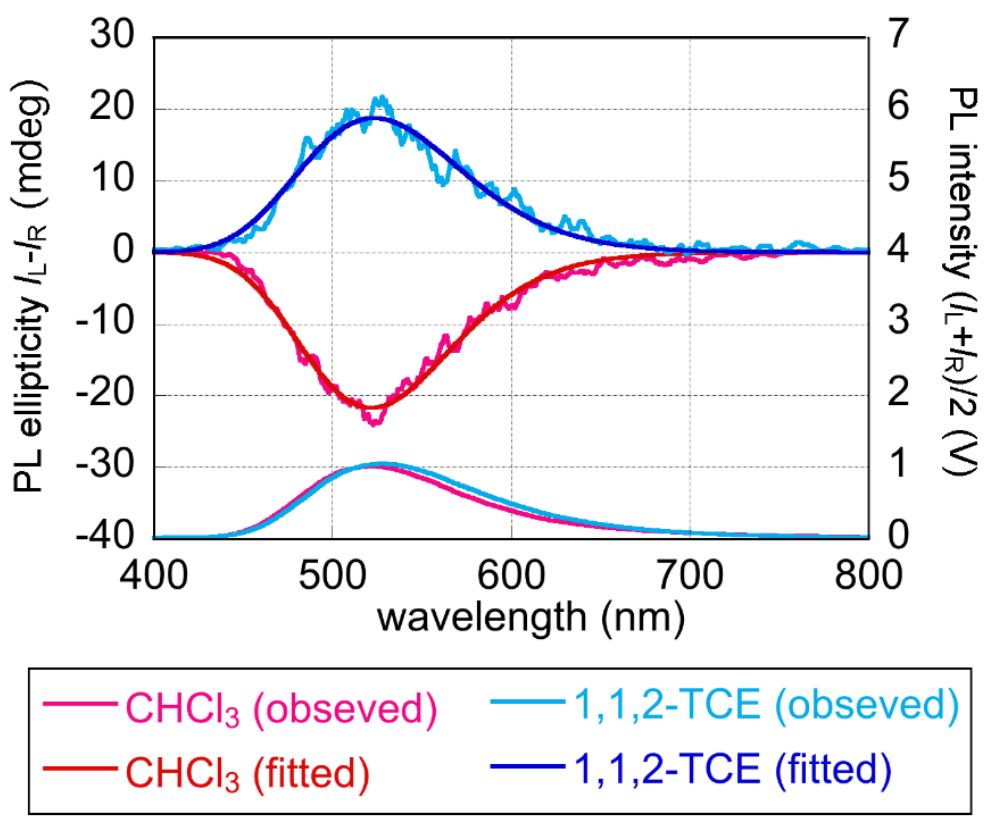

Figure S6. CPL and PL spectra of $\mathbf{P 1 b}(6 \mathbf{6 0})\left(2.80 \times 10^{-3} \mathrm{~g} / \mathrm{L}\right)$ in $\mathrm{CHCl}_{3}$ and 1,1,2-TCE $(\lambda \mathrm{ex}=291.0 \mathrm{~nm})$. 


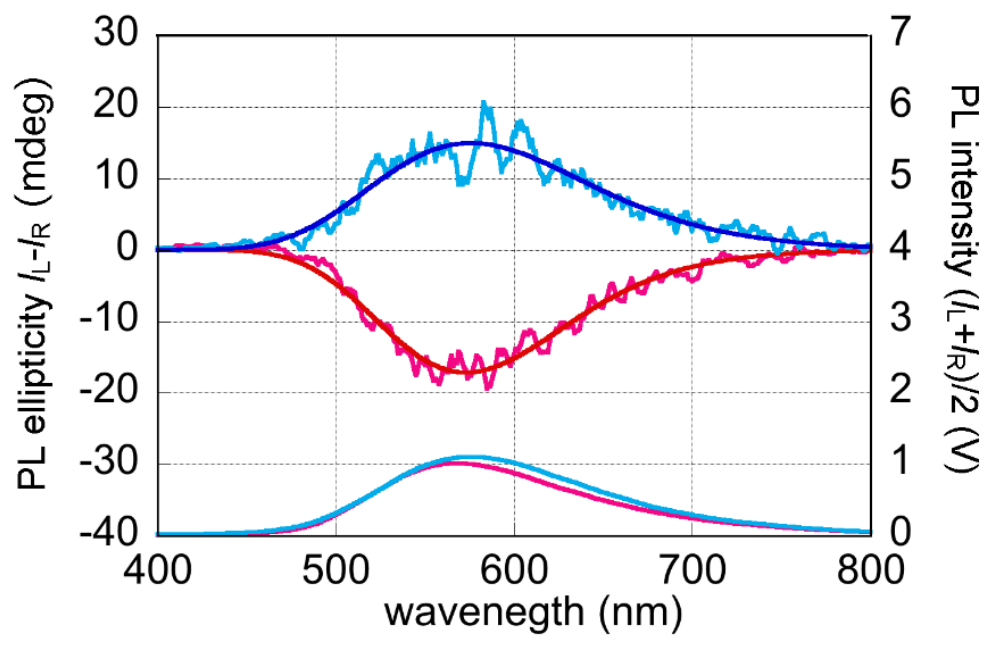

$-\mathrm{CHCl}_{3}$ (observed) $-1,1,2-\mathrm{TCE}$ (observed)
$-\mathrm{CHCl}_{3}$ (fitted) $-1,1,2-\mathrm{TCE}$ (fitted)

Figure S7. CPL and PL spectra of P1c(60) $\left(3.23 \times 10^{-3} \mathrm{~g} / \mathrm{L}\right)$ in $\mathrm{CHCl}_{3}$ and 1,1,2-TCE $(\lambda \mathrm{ex}=290.0 \mathrm{~nm})$.
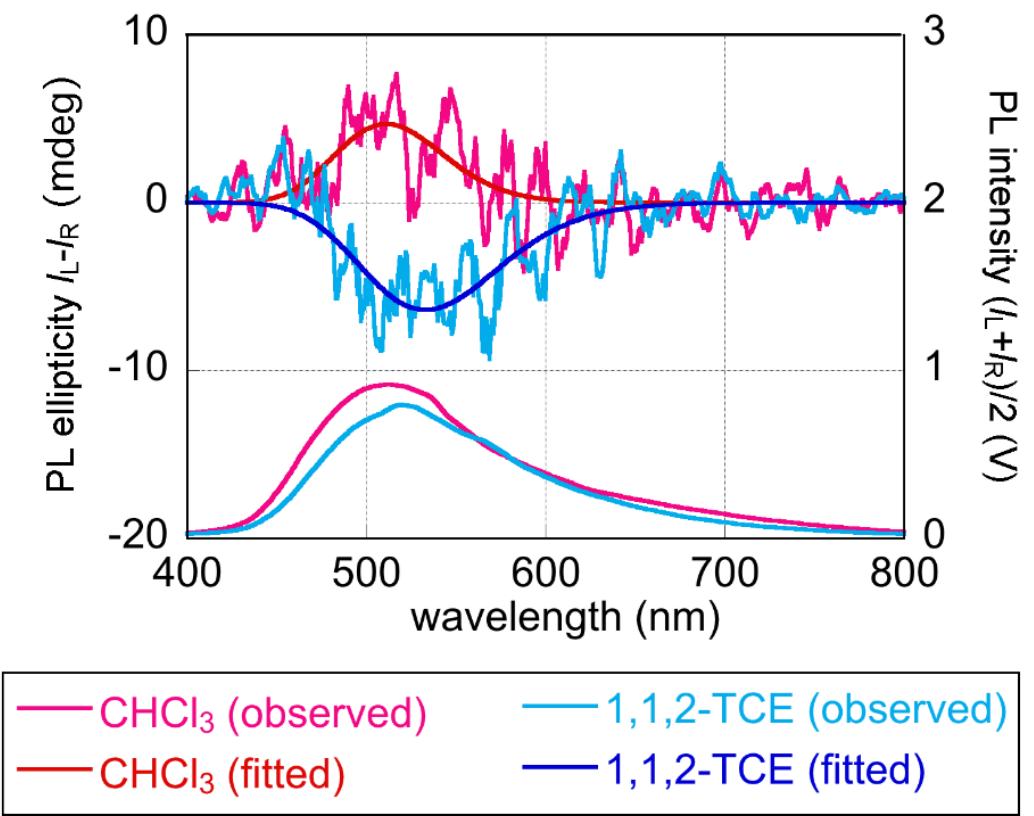

Figure S8. CPL and PL spectra of P2a(40) $\left(1.75 \times 10^{-3} \mathrm{~g} / \mathrm{L}\right)$ in $\mathrm{CHCl}_{3}$ and $1,1,2$-TCE $(\lambda \mathrm{ex}=291.0 \mathrm{~nm})$. 


\section{X-ray Crystallography}

Single-crystals of $\mathbf{1 c} \cdot 2 \mathrm{C}_{4} \mathrm{H}_{10} \mathrm{O}, \mathbf{2 a}, \mathbf{2 b}, \mathbf{6 a} \cdot \mathrm{C}_{4} \mathrm{H}_{8} \mathrm{O}, 7 \mathbf{a} \cdot \mathrm{C}_{6} \mathrm{H}_{14} \cdot 0.5 \mathrm{C}_{6} \mathrm{H}_{6}$, and $\mathbf{S 3}$ were obtained from THF/Et $2 \mathrm{O}$ (for $\mathbf{1 c} \cdot 2 \mathrm{C}_{4} \mathrm{H}_{10} \mathrm{O}$, and $\mathbf{6 a} \cdot \mathrm{C}_{4} \mathrm{H}_{8} \mathrm{O}$ ), $\mathrm{CH}_{2} \mathrm{Cl}_{2} /$ hexane (for $\mathbf{2 a}$ and 2b), $\mathrm{C}_{6} \mathrm{H}_{6} /$ hexane (for $7 \mathbf{a} \cdot \mathrm{C}_{6} \mathrm{H}_{14} \cdot 0.5 \mathrm{C}_{6} \mathrm{H}_{6}$ ), and $\mathrm{CH}_{2} \mathrm{Cl}_{2} / \mathrm{MeOH}$ (for $\mathbf{S 3}$ ) solutions and were mounted in the loop. The data for $1 \mathbf{c} \cdot 2 \mathrm{C}_{4} \mathrm{H}_{10} \mathrm{O}, \mathbf{6 a} \cdot \mathrm{C}_{4} \mathrm{H}_{8} \mathrm{O}$, and $7 \mathbf{a} \cdot \mathrm{C}_{6} \mathrm{H}_{14} \cdot 0.5 \mathrm{C}_{6} \mathrm{H}_{6}$ and were collected on a Rigaku R-AXIS RAPID-F graphite-monochromated Mo Ka radiation $(\lambda=0.71075 \AA)$ operating at $50 \mathrm{kV}$ and $40 \mathrm{~mA}$ at $-180{ }^{\circ} \mathrm{C}$ with imaging plate. The data for $\mathbf{S 3}$ were collected on a Rigaku Pilatus 200K graphite-monochromated Mo $\mathrm{K} \alpha$ radiation $(\lambda=0.71075 \AA)$ operating at $50 \mathrm{kV}$ and $24 \mathrm{~mA}$ at $-180{ }^{\circ} \mathrm{C}$ with imaging plate. The crystal data of $\mathbf{2 a}$ and $\mathbf{2} \mathbf{b}$ were collected at the BL40XU beamline of the SPring8 with the approval of the Japan Synchrotron Radiation Research Institute (JASRI), using a Rigaku Saturn724 CCD detector and $\mathrm{Si}(111)$-monochromated X-ray radiation $(\lambda=$ $0.78238 \AA$ ) at $-180{ }^{\circ} \mathrm{C}$. Data were processed with direct methods (SIR97 ${ }^{6}$ for $\mathbf{2 a}$ and $\mathbf{2 b}$, SIR $2014^{7}$ for $1 \mathbf{1 c} \cdot 2 \mathrm{C}_{4} \mathrm{H}_{10} \mathrm{O}, \mathbf{6 a} \cdot \mathrm{C}_{4} \mathrm{H}_{8} \mathrm{O}, 7 \mathbf{7} \cdot \mathrm{C}_{6} \mathrm{H}_{14} \cdot 0.5 \mathrm{C}_{6} \mathrm{H}_{6}$, and $\mathbf{S 3}$ ) and subsequent Fourier syntheses (SHELXL Version 2014/7 2b; Version2017/1 for $\mathbf{1 c} \cdot 2 \mathrm{C}_{4} \mathrm{H}_{10} \mathrm{O}$, 2a, 6a. $\left.\mathrm{C}_{4} \mathrm{H}_{8} \mathrm{O}, 7 \mathbf{7} \cdot \mathrm{C}_{6} \mathrm{H}_{14} \cdot 0.5 \mathrm{C}_{6} \mathrm{H}_{6}, \mathbf{S 3}\right) .{ }^{8}$ Empirical absorption correction was applied to all crystals. ${ }^{9}$ All non-hydrogen atoms were refined anisotropically except for a few solvated molecules (C95-C102, O1-O2 for $1 \mathbf{c} \cdot 2 \mathrm{C}_{4} \mathrm{H}_{10} \mathrm{O}$; C91-C99 for $\left.7 \mathbf{a} \cdot \mathrm{C}_{6} \mathrm{H}_{14} \cdot 0.5 \mathrm{C}_{6} \mathrm{H}_{6}\right)$. The positions of hydrogen atoms were calculated, and their contributions in structural factor calculations were included. All calculations were performed by using Yadokari-XG (Rev. 979). ${ }^{10}$ Crystallographic data of $\mathbf{1 c} \cdot 2 \mathrm{C}_{4} \mathrm{H}_{10} \mathrm{O}, \mathbf{2 a}, \mathbf{2 b}, \mathbf{6 a} \cdot \mathrm{C}_{4} \mathrm{H}_{8} \mathrm{O}, 7 \mathbf{a} \cdot \mathrm{C}_{6} \mathrm{H}_{14} \cdot 0.5 \mathrm{C}_{6} \mathrm{H}_{6}$, and S3 are summarized in Tables S5-10, and their ORTEP drawings are shown in Figures S914.

For the crystallographic data of $1 \mathrm{c} \cdot 2 \mathrm{C}_{4} \mathrm{H}_{10} \mathrm{O}$, hydrogen atoms on solvent molecules were not refined properly, thus alert level B error (PLAT043_ALERT_1_B Calculated and Reported Mol. Weight Differ by 20.1) is not avoidable. 
Table S5. X-ray crystallographic data for $1 \mathbf{c} \cdot 2 \mathrm{C}_{4} \mathrm{H}_{10} \mathrm{O}$.

\begin{tabular}{|c|c|c|}
\hline Empirical formula & \multicolumn{2}{|c|}{$\mathrm{C}_{102} \mathrm{H}_{108} \mathrm{Cl}_{2} \mathrm{~N}_{6} \mathrm{O}_{2} \mathrm{P}_{4} \mathrm{Pd}_{2}$} \\
\hline Formula weight & \multicolumn{2}{|l|}{1921.64} \\
\hline Crystal system & \multicolumn{2}{|l|}{ Triclinic } \\
\hline Space group & \multicolumn{2}{|l|}{$P \overline{1}$} \\
\hline \multirow[t]{3}{*}{ Unit cell dimension } & $a=14.5738(4) \AA$ & $\alpha=81.0382(8)^{\circ}$ \\
\hline & $b=18.0601(5) \AA$ & $\beta=77.9769(8)^{\circ}$ \\
\hline & $c=18.5802(5) \AA$ & $\gamma=89.6204(9)^{\circ}$ \\
\hline Volume & \multicolumn{2}{|l|}{$4723.0(2) \AA^{3}$} \\
\hline$Z$ & \multicolumn{2}{|l|}{2} \\
\hline Density (calculated) & \multicolumn{2}{|l|}{$1.351 \mathrm{~g} \mathrm{~cm}^{-3}$} \\
\hline Absorption coefficient & \multicolumn{2}{|l|}{$0.601 \mathrm{~mm}^{-1}$} \\
\hline$F(000)$ & \multicolumn{2}{|l|}{1992.0} \\
\hline Crystal size & \multicolumn{2}{|c|}{$0.40 \times 0.30 \times 0.25 \mathrm{~mm}^{3}$} \\
\hline Theta range for data collection & \multicolumn{2}{|l|}{2.995 to $27.5^{\circ}$} \\
\hline Index ranges & \multicolumn{2}{|c|}{$-18<=h<=18,-23<=k<=23,-24<=l<=24$} \\
\hline Reflection collected & \multicolumn{2}{|l|}{76247} \\
\hline Independent reflection & \multicolumn{2}{|c|}{$21554\left(R_{\text {int }}=0.0494\right)$} \\
\hline Completeness to theta $=27.5^{\circ}$ & \multicolumn{2}{|l|}{$99.5 \%$} \\
\hline Data / restrains / parameters & \multicolumn{2}{|l|}{$21554 / 0 / 1045$} \\
\hline Goodness-of -fit on $F^{2}$ & \multicolumn{2}{|l|}{1.088} \\
\hline Final $R$ indices $(I>2 \sigma(I))$ & \multicolumn{2}{|c|}{$R 1=0.0612, w R 2=0.1746$} \\
\hline$R$ indices (all data) & \multicolumn{2}{|c|}{$R 1=0.0747, w R 2=0.1916$} \\
\hline Largest diff. peak and hole, & \multicolumn{2}{|c|}{2.010 and -2.616 e $\AA^{-3}$} \\
\hline Weighting scheme & \multicolumn{2}{|c|}{$w=1 /\left[\sigma^{2}\left(F_{\mathrm{o}}^{2}\right)+(0.1124 \mathrm{P})^{2}+6.8460 \mathrm{P}\right]$} \\
\hline
\end{tabular}


Table S6. X-ray crystallographic data for $\mathbf{2 a}$.

\begin{tabular}{|c|c|c|}
\hline Empirical formula & \multicolumn{2}{|l|}{$\mathrm{C}_{66} \mathrm{H}_{48} \mathrm{Cl}_{2} \mathrm{~N}_{6}$} \\
\hline Formula weight & \multicolumn{2}{|l|}{996.00} \\
\hline Crystal system & \multicolumn{2}{|l|}{ Triclinic } \\
\hline Space group & \multicolumn{2}{|l|}{$P \overline{1}$} \\
\hline \multirow[t]{3}{*}{ Unit cell dimension } & $a=9.1275(3) \AA$ & $\alpha=104.902(2)^{\circ}$ \\
\hline & $b=17.0682(4) \AA$ & $\beta=98.456(2)^{\circ}$ \\
\hline & $c=17.2015(5) \AA$ & $\gamma=98.294(2)^{\circ}$ \\
\hline Volume & \multicolumn{2}{|l|}{ 2514.64(13) $\AA^{3}$} \\
\hline$Z$ & \multicolumn{2}{|l|}{2} \\
\hline Density (calculated) & \multicolumn{2}{|l|}{$1.315 \mathrm{~g} \mathrm{~cm}^{-3}$} \\
\hline Absorption coefficient & \multicolumn{2}{|l|}{$2.28 \mathrm{~mm}^{-1}$} \\
\hline$F(000)$ & \multicolumn{2}{|l|}{1040.0} \\
\hline Crystal size & \multicolumn{2}{|c|}{$0.50 \times 0.05 \times 0.05 \mathrm{~mm}^{3}$} \\
\hline Theta range for data collection & \multicolumn{2}{|l|}{1.385 to $27.5^{\circ}$} \\
\hline Index ranges & \multicolumn{2}{|c|}{$-10<=h<=10,-20<=k<=18,-20<=l<=20$} \\
\hline Reflection collected & \multicolumn{2}{|l|}{26615} \\
\hline Independent reflection & \multicolumn{2}{|c|}{$8653\left(R_{\text {int }}=0.0369\right)$} \\
\hline Completeness to theta $=27.5^{\circ}$ & \multicolumn{2}{|l|}{$99.8 \%$} \\
\hline Data / restrains / parameters & \multicolumn{2}{|l|}{$8653 / 0 / 673$} \\
\hline Goodness-of -fit on $F^{2}$ & \multicolumn{2}{|l|}{1.086} \\
\hline Final $R$ indices $(I>2 \sigma(I))$ & \multicolumn{2}{|c|}{$R 1=0.0598, w R 2=0.1467$} \\
\hline$R$ indices (all data) & \multicolumn{2}{|c|}{$R 1=0.070 ., w R 2=0.1535$} \\
\hline Largest diff. peak and hole, & \multicolumn{2}{|c|}{0.338 and -0.476 e $\AA^{-3}$} \\
\hline Weighting scheme & \multicolumn{2}{|c|}{$w=1 /\left[\sigma^{2}\left(F_{\mathrm{o}}^{2}\right)+(0.0642 \mathrm{P})^{2}+2.1845 \mathrm{P}\right]$} \\
\hline
\end{tabular}


Table S7. X-ray crystallographic data for $\mathbf{2 b}$.

\begin{tabular}{|c|c|c|}
\hline Empirical formula & \multicolumn{2}{|l|}{$\mathrm{C}_{66} \mathrm{H}_{48} \mathrm{Cl}_{2} \mathrm{~N}_{6} \mathrm{O}_{2}$} \\
\hline Formula weight & \multicolumn{2}{|l|}{1028.00} \\
\hline Crystal system & \multicolumn{2}{|l|}{ Triclinic } \\
\hline Space group & \multicolumn{2}{|l|}{$P \overline{1}$} \\
\hline \multirow[t]{3}{*}{ Unit cell dimension } & $a=9.1607(3) \AA$ & $\alpha=106.194(2)^{\circ}$ \\
\hline & $b=16.8571(5) \AA$ & $\beta=99.701(2)^{\circ}$ \\
\hline & $c=17.2193(5) \AA$ & $\gamma=96.486(2)^{\circ}$ \\
\hline Volume & \multicolumn{2}{|l|}{$2480.83(13) \AA^{3}$} \\
\hline$Z$ & \multicolumn{2}{|l|}{2} \\
\hline Density (calculated) & \multicolumn{2}{|l|}{$1.376 \mathrm{~g} \mathrm{~cm}^{-3}$} \\
\hline Absorption coefficient & \multicolumn{2}{|l|}{$2.38 \mathrm{~mm}^{-1}$} \\
\hline$F(000)$ & \multicolumn{2}{|l|}{1072.0} \\
\hline Crystal size & \multicolumn{2}{|c|}{$0.02 \times 0.02 \times 0.02 \mathrm{~mm}^{3}$} \\
\hline Theta range for data collection & \multicolumn{2}{|l|}{1.386 to $27.500^{\circ}$} \\
\hline Index ranges & \multicolumn{2}{|c|}{$-10<=h<=10,-19<=k<=19,-20<=l<=20$} \\
\hline Reflection collected & \multicolumn{2}{|l|}{26560} \\
\hline Independent reflection & \multicolumn{2}{|c|}{$8465\left(R_{\text {int }}=0.0780\right)$} \\
\hline Completeness to theta $=27.500^{\circ}$ & \multicolumn{2}{|l|}{$99.1 \%$} \\
\hline Data / restrains / parameters & \multicolumn{2}{|l|}{8465 / 0 / 691} \\
\hline Goodness-of -fit on $F^{2}$ & \multicolumn{2}{|l|}{1.100} \\
\hline Final $R$ indices $(I>2 \sigma(I))$ & \multicolumn{2}{|c|}{$R 1=0.0535, w R 2=0.1153$} \\
\hline$R$ indices (all data) & \multicolumn{2}{|c|}{$R 1=0.1083, w R 2=0.1603$} \\
\hline Largest diff. peak and hole, & \multicolumn{2}{|c|}{0.517 and -0.498 e $\AA^{-3}$} \\
\hline Weighting scheme & \multicolumn{2}{|c|}{$w=1 /\left[\sigma^{2}\left(F_{\mathrm{o}}^{2}\right)+(0.0558 \mathrm{P})^{2}+2.3182 \mathrm{P}\right]$} \\
\hline
\end{tabular}


Table S8. X-ray crystallographic data for $\mathbf{6 a} \cdot \mathrm{C}_{4} \mathrm{H}_{8} \mathrm{O}$.

\begin{tabular}{|c|c|c|}
\hline Empirical formula & \multicolumn{2}{|l|}{$\mathrm{C}_{86} \mathrm{H}_{78} \mathrm{Cl}_{2} \mathrm{~N}_{6} \mathrm{OP}_{2} \mathrm{Pd}$} \\
\hline Formula weight & \multicolumn{2}{|l|}{1450.78} \\
\hline Crystal system & \multicolumn{2}{|l|}{ Triclinic } \\
\hline Space group & \multicolumn{2}{|l|}{$P \overline{1}$} \\
\hline \multirow[t]{3}{*}{ Unit cell dimension } & $a=12.5902(6) \AA$ & $\alpha=83.7616(13)^{\circ}$ \\
\hline & $b=15.6843(6) \AA$ & $\beta=74.8828(15)^{\circ}$ \\
\hline & $c=19.3156(8) \AA$ & $\gamma=76.0836(16)^{\circ}$ \\
\hline Volume & \multicolumn{2}{|l|}{$3569.8(3) \AA^{3}$} \\
\hline$Z$ & \multicolumn{2}{|l|}{2} \\
\hline Density (calculated) & \multicolumn{2}{|l|}{$1.350 \mathrm{~g} \mathrm{~cm}^{-3}$} \\
\hline Absorption coefficient & \multicolumn{2}{|l|}{$0.433 \mathrm{~mm}^{-1}$} \\
\hline$F(000)$ & \multicolumn{2}{|l|}{1508.0} \\
\hline Crystal size & \multicolumn{2}{|c|}{$0.50 \times 0.20 \times 0.10 \mathrm{~mm}^{3}$} \\
\hline Theta range for data collection & \multicolumn{2}{|l|}{3.002 to $27.5^{\circ}$} \\
\hline Index ranges & \multicolumn{2}{|c|}{$-16<=h<=16,-20<=k<=20,-25<=l<=25$} \\
\hline Reflection collected & \multicolumn{2}{|l|}{57673} \\
\hline Independent reflection & \multicolumn{2}{|c|}{$16337\left(R_{\mathrm{int}}=0.0580\right)$} \\
\hline Completeness to theta $=27.5^{\circ}$ & \multicolumn{2}{|l|}{$99.8 \%$} \\
\hline Data / restrains / parameters & \multicolumn{2}{|l|}{$16337 / 0 / 893$} \\
\hline Goodness-of -fit on $F^{2}$ & \multicolumn{2}{|l|}{1.068} \\
\hline Final $R$ indices $(I>2 \sigma(I))$ & \multicolumn{2}{|c|}{$R 1=0.0457, w R 2=0.1222$} \\
\hline$R$ indices (all data) & \multicolumn{2}{|c|}{$R 1=0.0603, w R 2=0.1350$} \\
\hline Largest diff. peak and hole, & \multicolumn{2}{|c|}{1.021 and $-1.530 \mathrm{e} \AA^{-3}$} \\
\hline Weighting scheme & \multicolumn{2}{|c|}{$w=1 /\left[\sigma^{2}\left(F_{\mathrm{o}}^{2}\right)+(0.0690 \mathrm{P})^{2}+3.2447 \mathrm{P}\right]$} \\
\hline
\end{tabular}


Table S9. X-ray crystallographic data for $7 \mathbf{a} \cdot \mathrm{C}_{6} \mathrm{H}_{14} \cdot 0.5 \mathrm{C}_{6} \mathrm{H}_{6}$.

\begin{tabular}{|c|c|c|}
\hline Empirical formula & \multicolumn{2}{|l|}{$\mathrm{C}_{99} \mathrm{H}_{98} \mathrm{Cl}_{2} \mathrm{~N}_{6} \mathrm{P}_{3} \mathrm{Pd}_{2}$} \\
\hline Formula weight & \multicolumn{2}{|l|}{1748.44} \\
\hline Crystal system & \multicolumn{2}{|l|}{ Monoclinic } \\
\hline Space group & \multicolumn{2}{|l|}{$P 2_{1} / n$} \\
\hline \multirow[t]{3}{*}{ Unit cell dimension } & $a=14.9006(4) \AA$ & $\alpha=90^{\circ}$ \\
\hline & $b=26.7100(6) \AA$ & $\beta=101.1041(8)^{\circ}$ \\
\hline & $c=21.7090(5) \AA$ & $\gamma=90^{\circ}$ \\
\hline Volume & \multicolumn{2}{|l|}{$8478.3(4) \AA^{3}$} \\
\hline$Z$ & \multicolumn{2}{|l|}{4} \\
\hline Density (calculated) & \multicolumn{2}{|l|}{$1.370 \mathrm{~g} \mathrm{~cm}^{-3}$} \\
\hline Absorption coefficient & \multicolumn{2}{|l|}{$0.596 \mathrm{~mm}^{-1}$} \\
\hline$F(000)$ & \multicolumn{2}{|l|}{3620.0} \\
\hline Crystal size & \multicolumn{2}{|c|}{$0.50 \times 0.30 \times 0.15 \mathrm{~mm}^{3}$} \\
\hline Theta range for data collection & \multicolumn{2}{|l|}{3.035 to $27.5^{\circ}$} \\
\hline Index ranges & \multicolumn{2}{|c|}{$-19<=h<=19,-29<=k<=34,-28<=l<=28$} \\
\hline Reflection collected & \multicolumn{2}{|l|}{83214} \\
\hline Independent reflection & \multicolumn{2}{|c|}{$19418\left(R_{\mathrm{int}}=0.0741\right)$} \\
\hline Completeness to theta $=27.5^{\circ}$ & \multicolumn{2}{|l|}{$99.9 \%$} \\
\hline Data / restrains / parameters & \multicolumn{2}{|l|}{19418 / 0 / 976} \\
\hline Goodness-of -fit on $F^{2}$ & \multicolumn{2}{|l|}{1.040} \\
\hline Final $R$ indices $(I>2 \sigma(I))$ & \multicolumn{2}{|c|}{$R 1=0.0564, w R 2=0.1266$} \\
\hline$R$ indices (all data) & \multicolumn{2}{|c|}{$R 1=0.0764, w R 2=0.1369$} \\
\hline Largest diff. peak and hole, & \multicolumn{2}{|c|}{2.063 and $-1.020 \mathrm{e} \AA^{-3}$} \\
\hline Weighting scheme & \multicolumn{2}{|c|}{$w=1 /\left[\sigma^{2}\left(F_{\mathrm{o}}^{2}\right)+(0.0485 \mathrm{P})^{2}+24.7385 \mathrm{P}\right]$} \\
\hline
\end{tabular}


Table S10. X-ray crystallographic data for S3.

\begin{tabular}{|c|c|c|}
\hline Empirical formula & \multicolumn{2}{|l|}{$\mathrm{C}_{46} \mathrm{H}_{34} \mathrm{Cl}_{2} \mathrm{~N}_{4} \mathrm{O}_{2}$} \\
\hline Formula weight & \multicolumn{2}{|l|}{745.67} \\
\hline Crystal system & \multicolumn{2}{|l|}{ Triclinic } \\
\hline Space group & \multicolumn{2}{|l|}{$P \overline{1}$} \\
\hline \multirow[t]{3}{*}{ Unit cell dimension } & $a=9.341(4) \AA$ & $\alpha=107.362(6)^{\circ}$ \\
\hline & $b=12.431(5) \AA$ & $\beta=94.593(5)^{\circ}$ \\
\hline & $c=16.645(7) \AA$ & $\gamma=102.557(5)^{\circ}$ \\
\hline Volume & \multicolumn{2}{|l|}{$1778.7(13) \AA^{3}$} \\
\hline$Z$ & \multicolumn{2}{|l|}{2} \\
\hline Density (calculated) & \multicolumn{2}{|l|}{$1.392 \mathrm{~g} \mathrm{~cm}^{-3}$} \\
\hline Absorption coefficient & \multicolumn{2}{|l|}{$2.30 \mathrm{~mm}^{-1}$} \\
\hline$F(000)$ & \multicolumn{2}{|l|}{776.0} \\
\hline Crystal size & \multicolumn{2}{|c|}{$0.01 \times 0.01 \times 0.01 \mathrm{~mm}^{3}$} \\
\hline Theta range for data collection & \multicolumn{2}{|l|}{3.095 to $27.5^{\circ}$} \\
\hline Index ranges & \multicolumn{2}{|c|}{$-12<=h<=12,-16<=k<=16,-14<=l<=21$} \\
\hline Reflection collected & \multicolumn{2}{|l|}{15184} \\
\hline Independent reflection & \multicolumn{2}{|c|}{$7593\left(R_{\text {int }}=0.0406\right)$} \\
\hline Completeness to theta $=27.5^{\circ}$ & \multicolumn{2}{|l|}{$93.2 \%$} \\
\hline Data / restrains / parameters & \multicolumn{2}{|l|}{$7593 / 0$ / 491} \\
\hline Goodness-of -fit on $F^{2}$ & \multicolumn{2}{|l|}{1.053} \\
\hline Final $R$ indices $(I>2 \sigma(I))$ & \multicolumn{2}{|c|}{$R 1=0.0604, w R 2=0.1258$} \\
\hline$R$ indices (all data) & \multicolumn{2}{|c|}{$R 1=0.0863, w R 2=0.1478$} \\
\hline Largest diff. peak and hole, & \multicolumn{2}{|c|}{1.076 and -0.536 e $\AA^{-3}$} \\
\hline Weighting scheme & \multicolumn{2}{|c|}{$w=1 /\left[\sigma^{2}\left(F_{\mathrm{o}}^{2}\right)+(0.0447 \mathrm{P})^{2}+1.8230 \mathrm{P}\right]$} \\
\hline
\end{tabular}




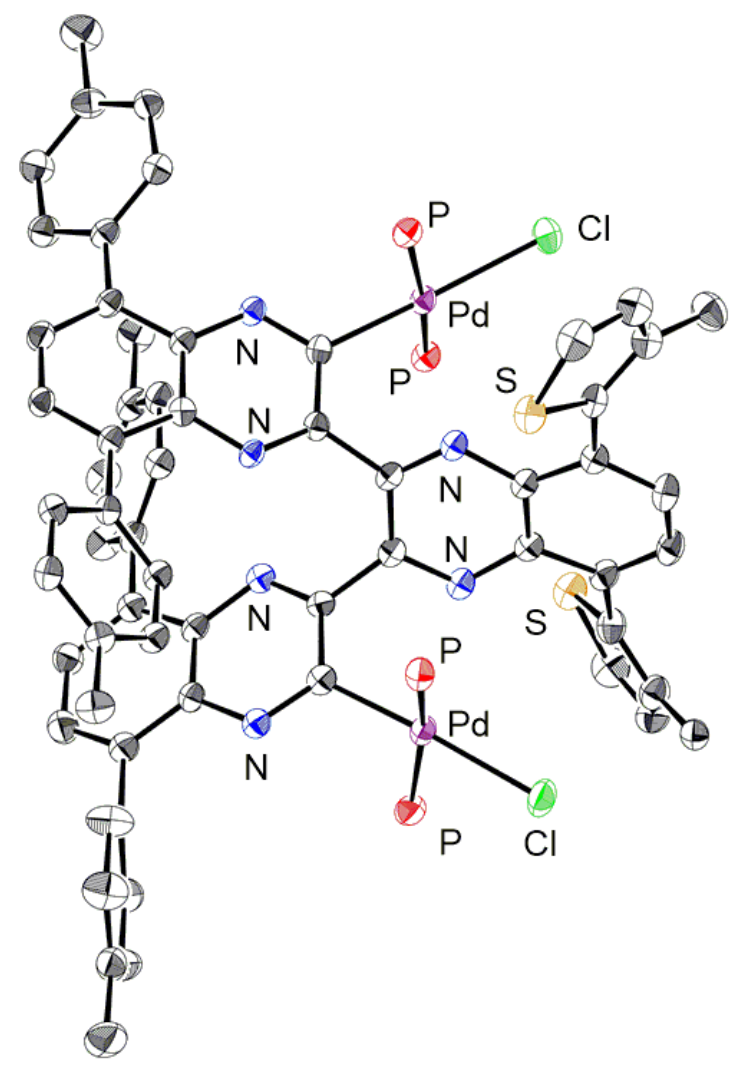

Figure S9. An ORTEP drawing of $1 \mathbf{c} \cdot 2 \mathrm{C}_{4} \mathrm{H}_{10} \mathrm{O}$. Substituents on phosphorous atoms, hydrogen atoms, and solvated molecules are omitted for clarity. 


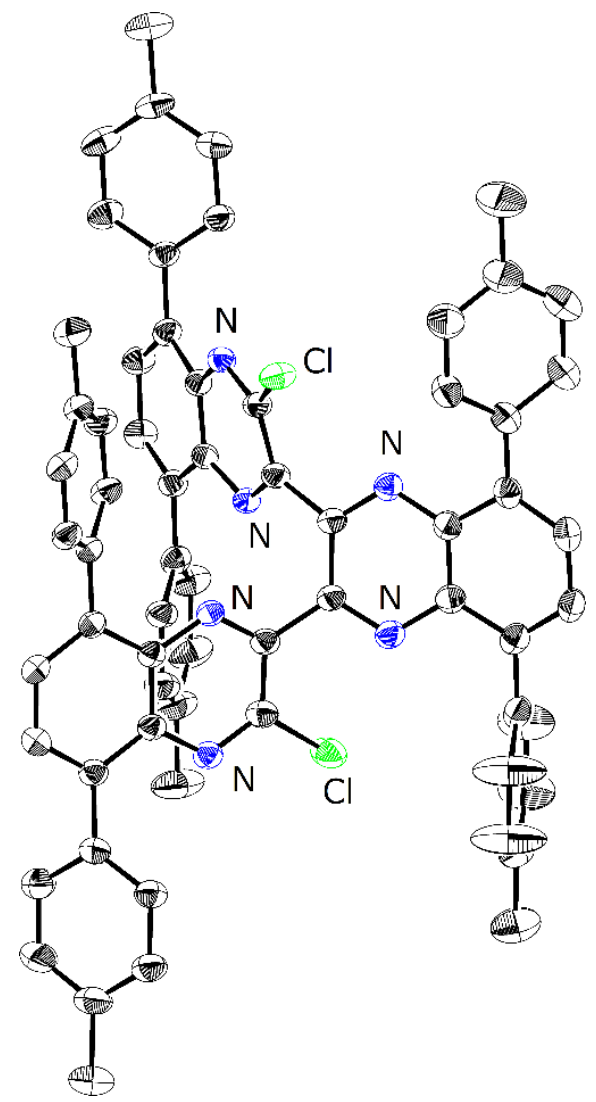

Figure S10. An ORTEP drawing of 2a. Hydrogen atoms are omitted for clarity. 


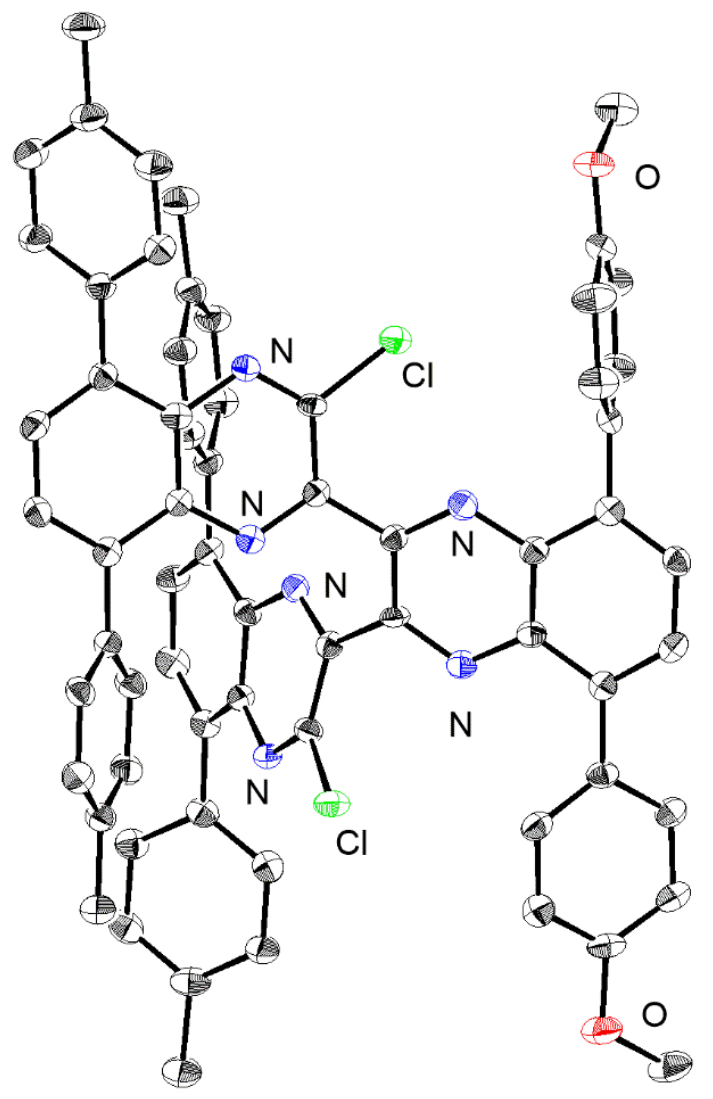

Figure S11. An ORTEP drawing of 2b. Hydrogen atoms are omitted for clarity. 


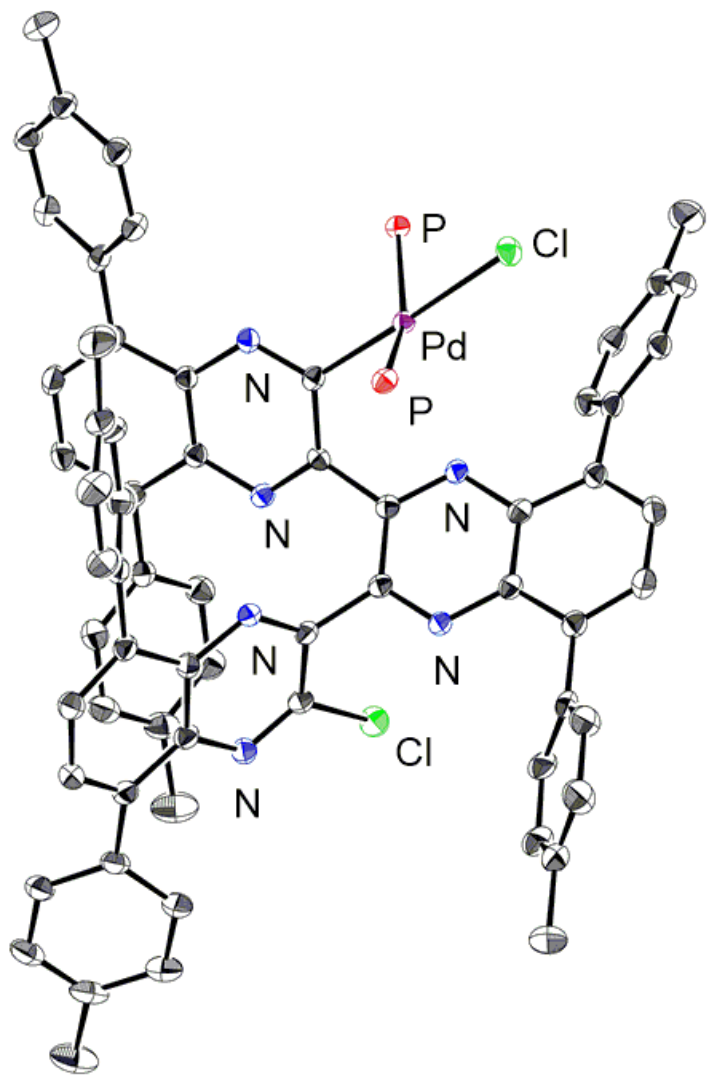

Figure S12. An ORTEP drawing of $\mathbf{6 a} \cdot \mathrm{C}_{4} \mathrm{H}_{8} \mathrm{O}$. Substituents on phosphorous atoms, hydrogen atoms, and solvated molecules are omitted for clarity. 


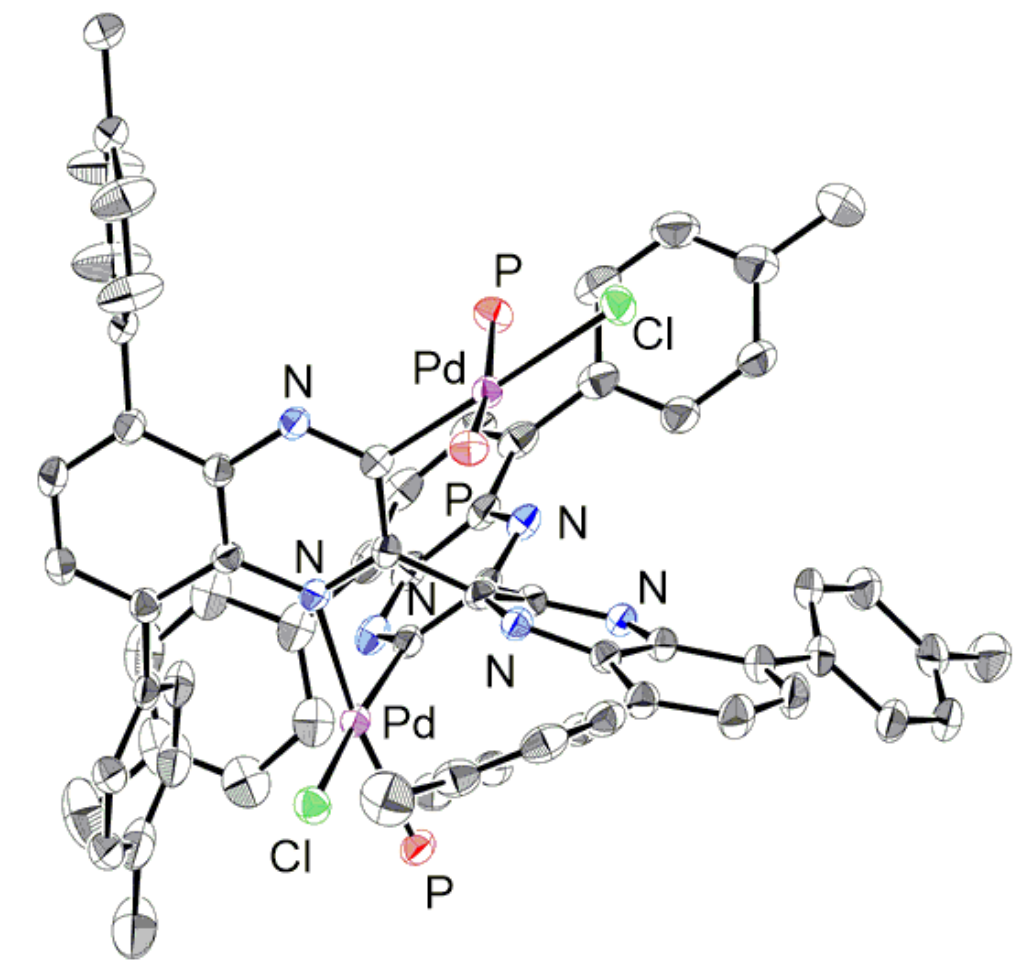

Figure S13. An ORTEP drawing of $7 \mathbf{a} \cdot \mathrm{C}_{6} \mathrm{H}_{14} \cdot 0.5 \mathrm{C}_{6} \mathrm{H}_{6}$. Substituents on phosphorous atoms, hydrogen atoms, and solvated molecules are omitted for clarity. 


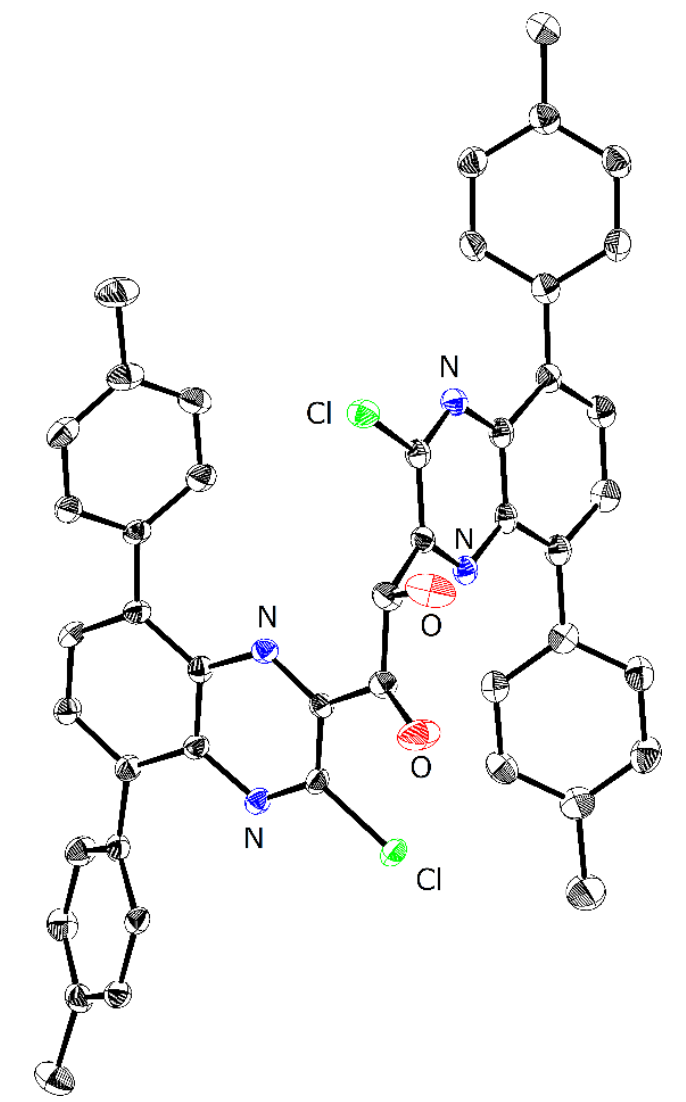

Figure S14. An ORTEP drawing of S3. Hydrogen atoms are omitted for clarity. 


\section{References}

(1) Yamada, T.; Nagata, Y.; Suginome, M. Chem. Commun. 2010, 46, 4914-4916.

(2) Zhu, Y.-Y.; Yin, T.-T.; Yin, J.; Liu, N.; Yu, Z.-P.; Zhu, Y.-W.; Ding, Y.-S.; Yin J.; Wu, Z.-Q. RSC Adv. 2014, 4, 40241-40250.

(3) Nishikawa, T.; Nagata, Y.; Suginome, M. ACS Macro Lett. 2017, 6, 431-435.

(4) Jiang, L.; Huang, Y.; Yan, Y.; Xie, Y. Tetrahedron Lett. 2016, 57, 4149-4151.

(5) Bonsall, S. D.; Houcheime, M.; Straus, D. A.; Muller, G. Chem. Commun. 2007, 35, 3676-3678.

(6) Altomare, A.; Burla, M. C.; Camalli, M.; Cascarano, G. L.; Giacovazzo, C.; Guagliardi, A.; Moliterni, A. G. G.; Polidori, G.; Spagna, R. J. Appl. Crystallogr. 1999, $32,115-119$.

(7) Burla, M. C.; Caliandro, R.; Carrozzini, B.; Cascarano, G. L.; Cuocci, C.; Giacovazzo,

C.; Mallamo, M.; Mazzone, A.; Polidori, G. J. Appl. Crystallogr. 2015, 48, 306-309.

(8) Sheldrick, G. M. Acta Cryst. A 2015, 71, 3-8.

(9) Blessing, R. H. Acta Cryst. A 1995, 51, 33-38.

(10) Kabuto, C.; Akine, S.; Nemoto, T.; Kwon, E. J. Cryst. Soc. Jpn. 2009, 51, 218-224. 
10 UV-vis, CD, and PL Spectra of New Compounds

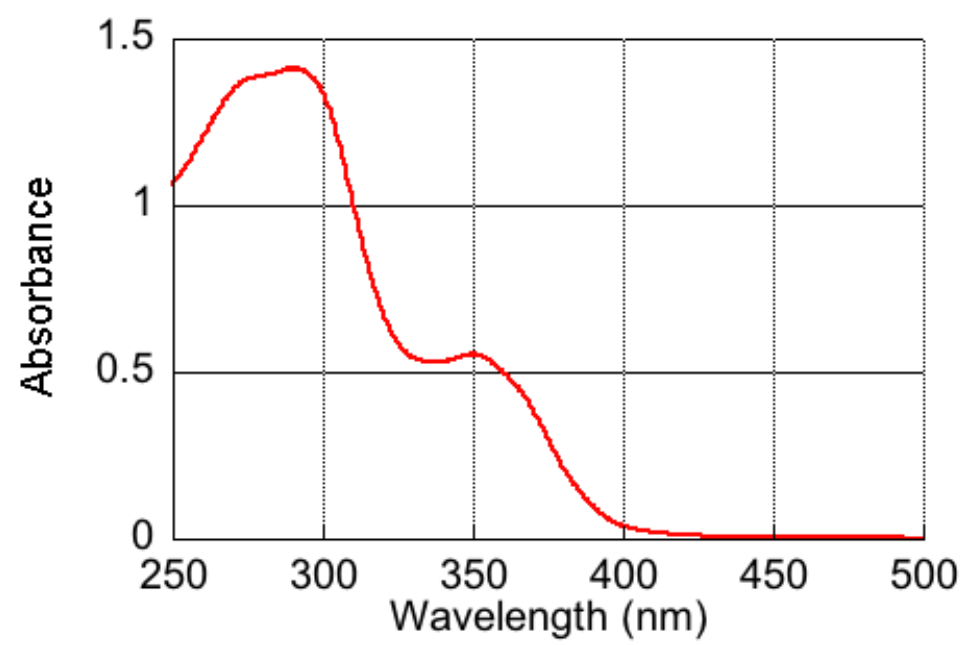

Figure S15. UV-vis absorption spectrum of $\mathbf{P 1 a ( 4 0 )}$ in $\mathrm{CHCl}_{3}$ $\left(2.60 \times 10^{-2} \mathrm{~g} / \mathrm{L}\right.$, light path length $\left.=10 \mathrm{~mm}\right)$.

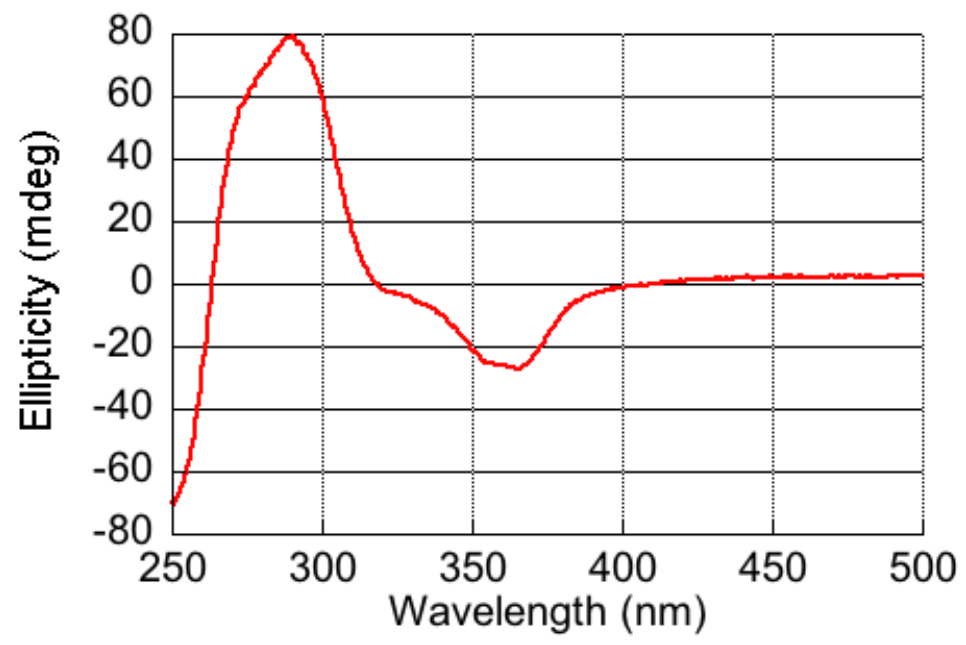

Figure S16. CD spectrum of $\mathbf{P 1 a}(40)$ in $\mathrm{CHCl}_{3}$ $\left(2.60 \times 10^{-2} \mathrm{~g} / \mathrm{L}\right.$, light path length $\left.=10 \mathrm{~mm}\right)$. 


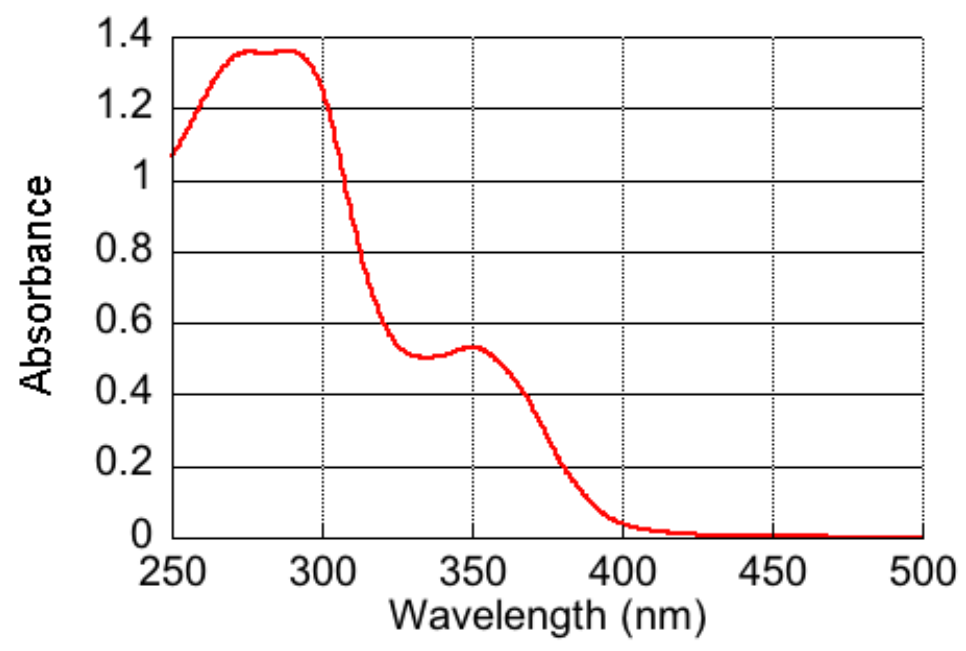

Figure S17. UV-vis absorption spectrum of P1a(40) in 1,1,2-TCE $\left(2.60 \times 10^{-2} \mathrm{~g} / \mathrm{L}\right.$, light path length $\left.=10 \mathrm{~mm}\right)$.

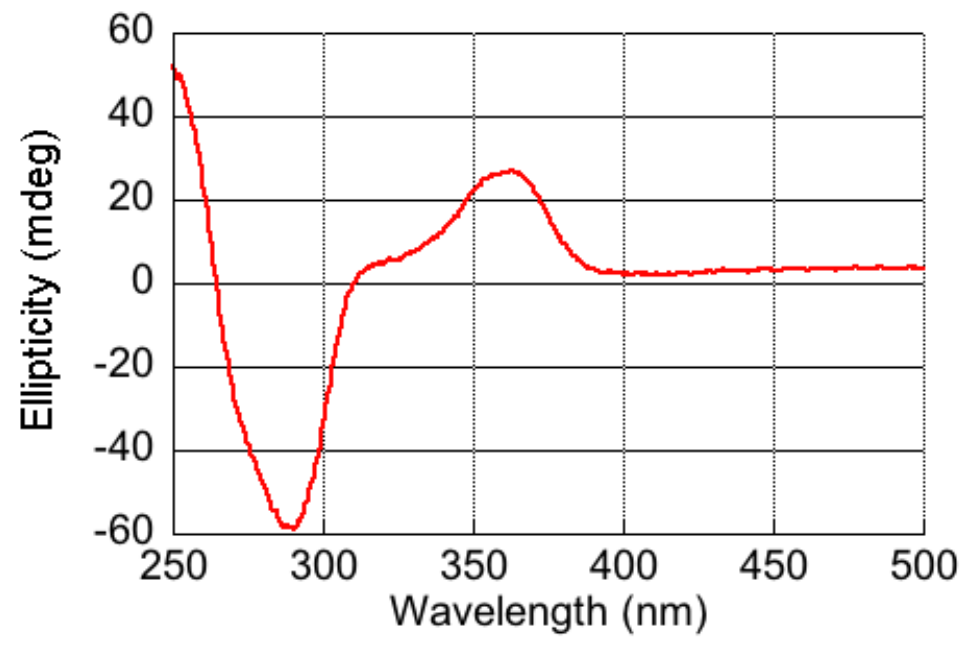

Figure S18. CD spectrum of P1a(40) in 1,1,2-TCE $\left(2.60 \times 10^{-2} \mathrm{~g} / \mathrm{L}\right.$, light path length $\left.=10 \mathrm{~mm}\right)$. 


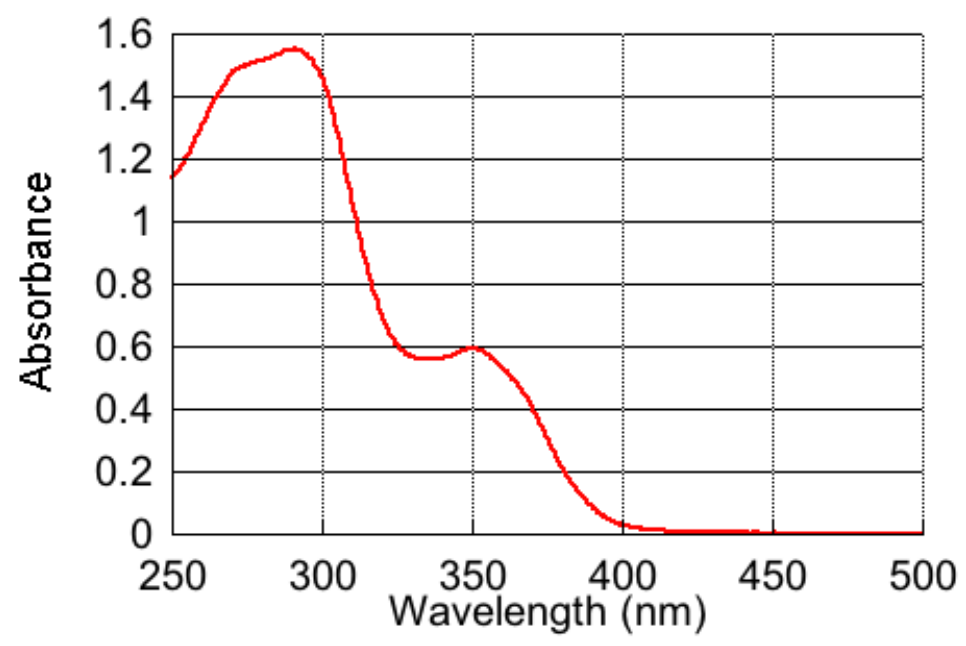

Figure S19. UV-vis absorption spectrum of $\mathbf{P 1 a ( 6 0 )}$ in $\mathrm{CHCl}_{3}$ $\left(2.86 \times 10^{-2} \mathrm{~g} / \mathrm{L}\right.$, light path length $\left.=10 \mathrm{~mm}\right)$.

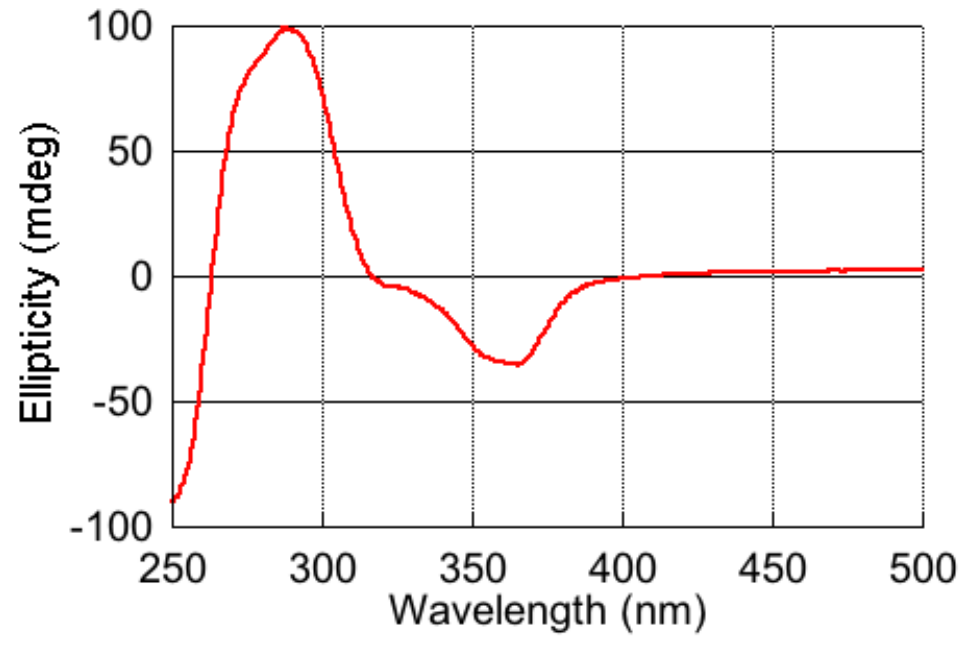

Figure S20. CD spectrum of $\mathbf{P 1 a ( 6 0 )}$ in $\mathrm{CHCl}_{3}$ $\left(2.86 \times 10^{-2} \mathrm{~g} / \mathrm{L}\right.$, light path length $\left.=10 \mathrm{~mm}\right)$. 


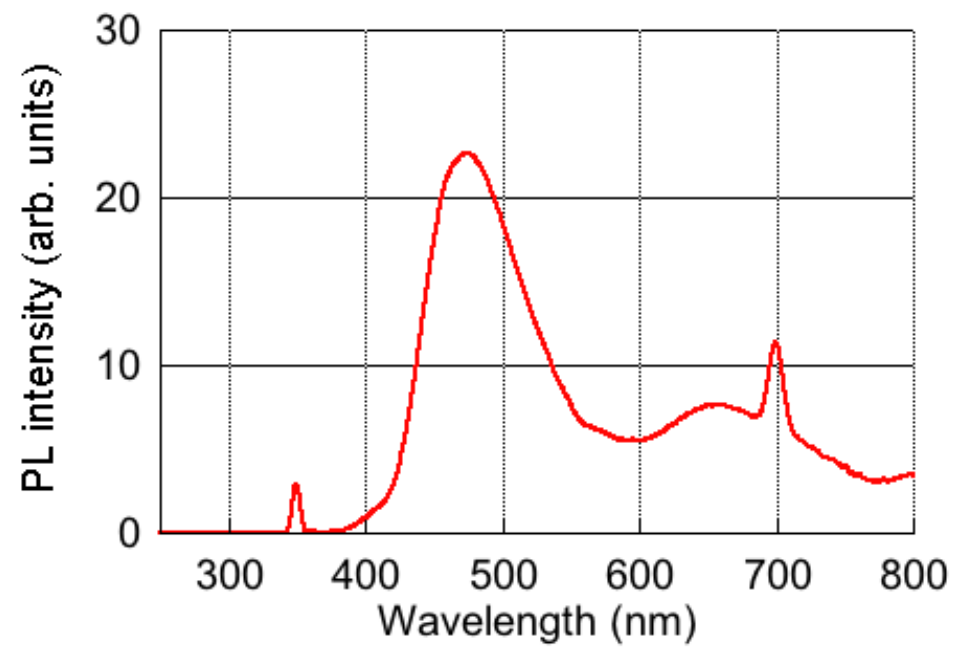

Figure S21. PL spectrum of P1a(60) in $\mathrm{CHCl}_{3}$ $\left(2.86 \times 10^{-2} \mathrm{~g} / \mathrm{L}, \lambda_{\mathrm{ex}}=349.5 \mathrm{~nm}\right)$.

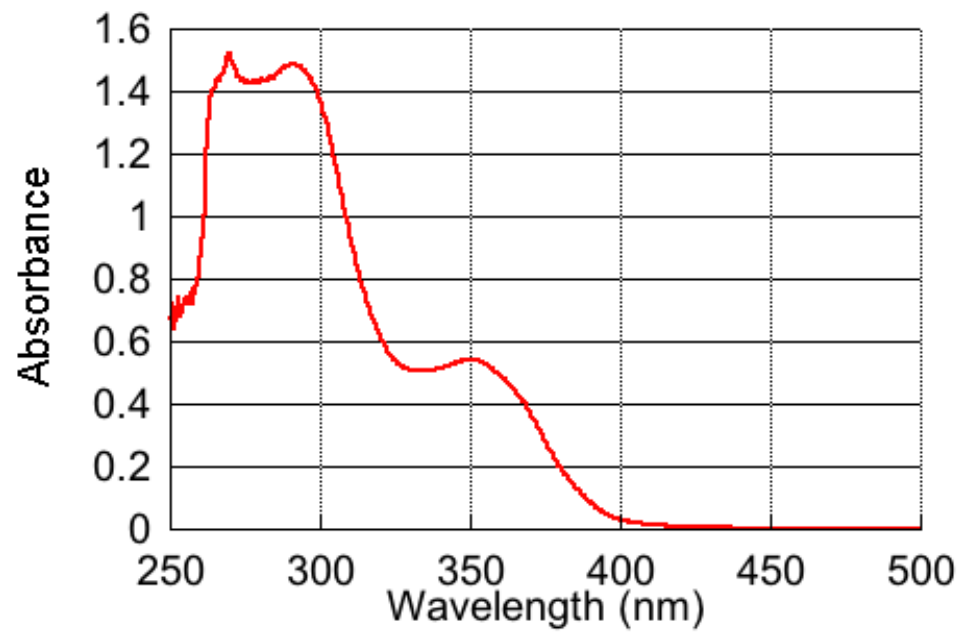

Figure S22. UV-vis absorption spectrum of P1a(60) in 1,1,2-TCE $\left(2.86 \times 10^{-2} \mathrm{~g} / \mathrm{L}\right.$, light path length $\left.=10 \mathrm{~mm}\right)$. 


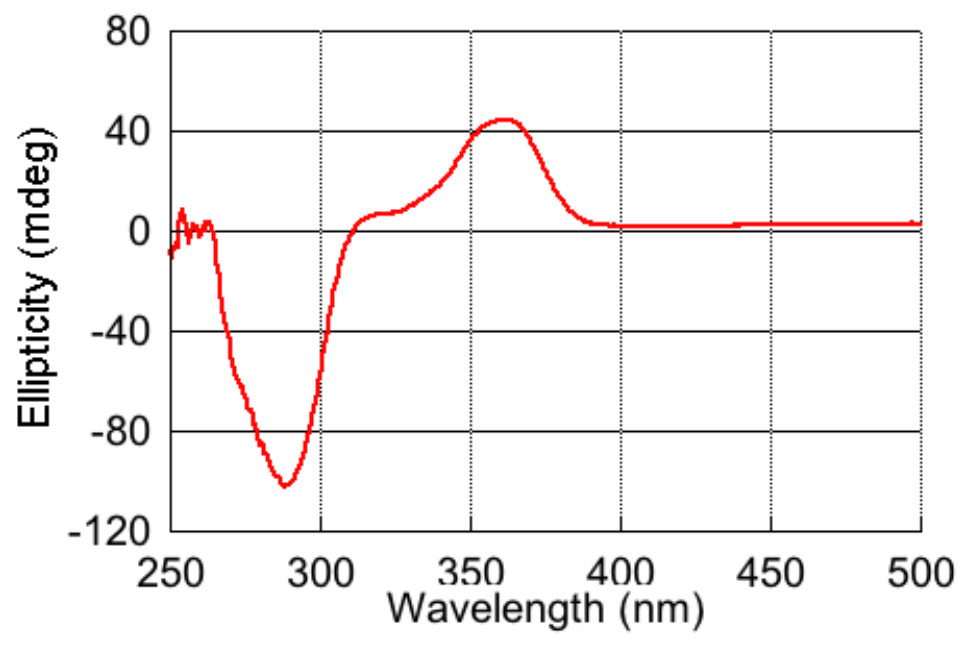

Figure S23. CD spectrum of $\mathbf{P 1 a}(\mathbf{6 0})$ in 1,1,2-TCE

$\left(2.86 \times 10^{-2} \mathrm{~g} / \mathrm{L}\right.$, light path length $\left.=10 \mathrm{~mm}\right)$.

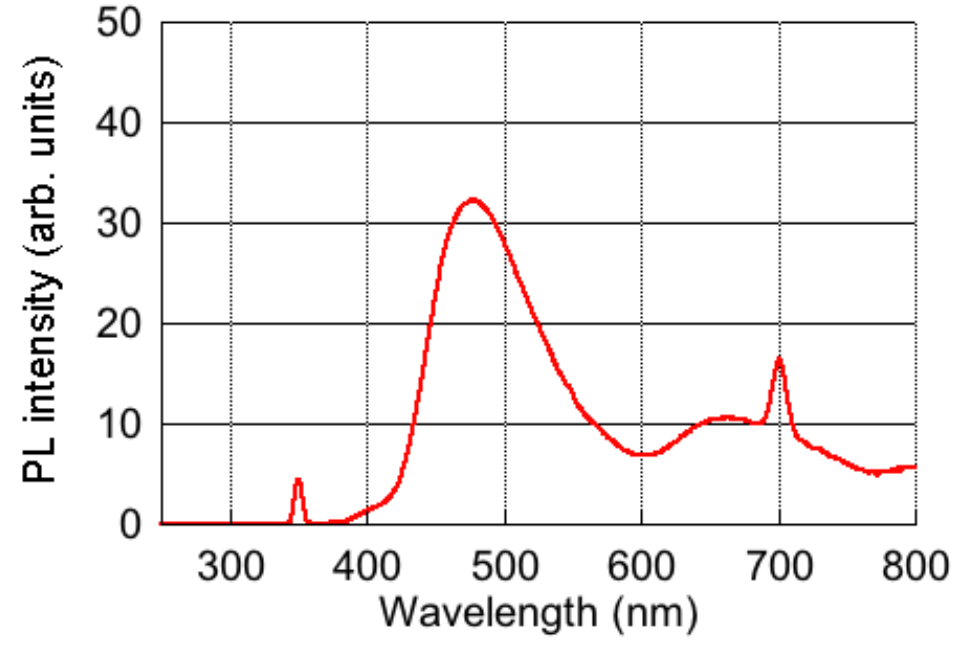

Figure S24. PL spectrum of P1a(60) in 1,1,2-TCE $\left(2.86 \times 10^{-2} \mathrm{~g} / \mathrm{L}, \lambda_{\mathrm{ex}}=349.5 \mathrm{~nm}\right)$. 


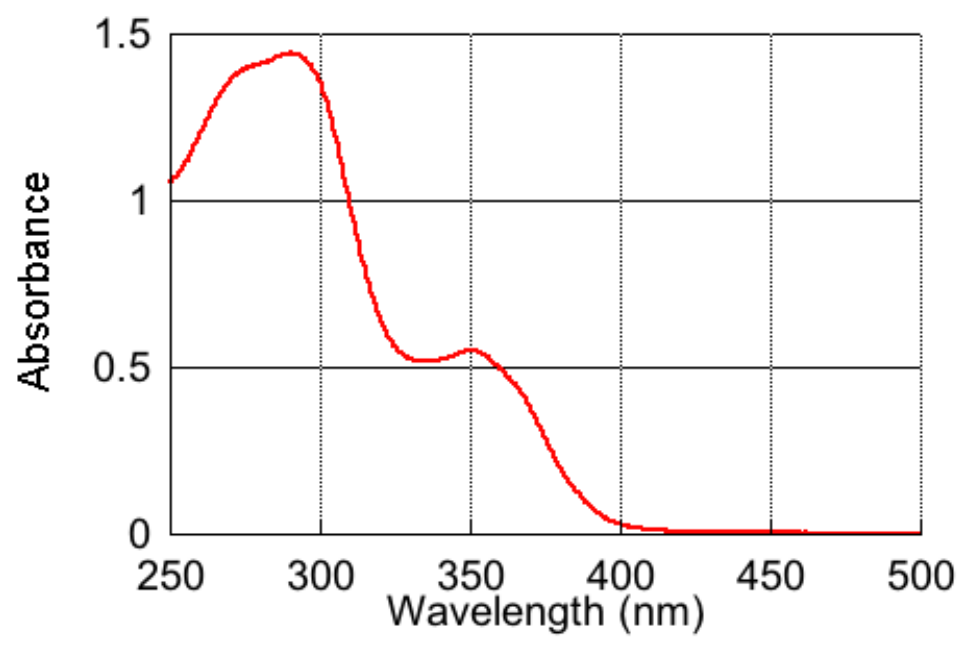

Figure S25. UV-vis absorption spectrum of $\mathbf{P 1 a ( 8 0 )}$ in $\mathrm{CHCl}_{3}$ $\left(3.00 \times 10^{-2} \mathrm{~g} / \mathrm{L}\right.$, light path length $\left.=10 \mathrm{~mm}\right)$.

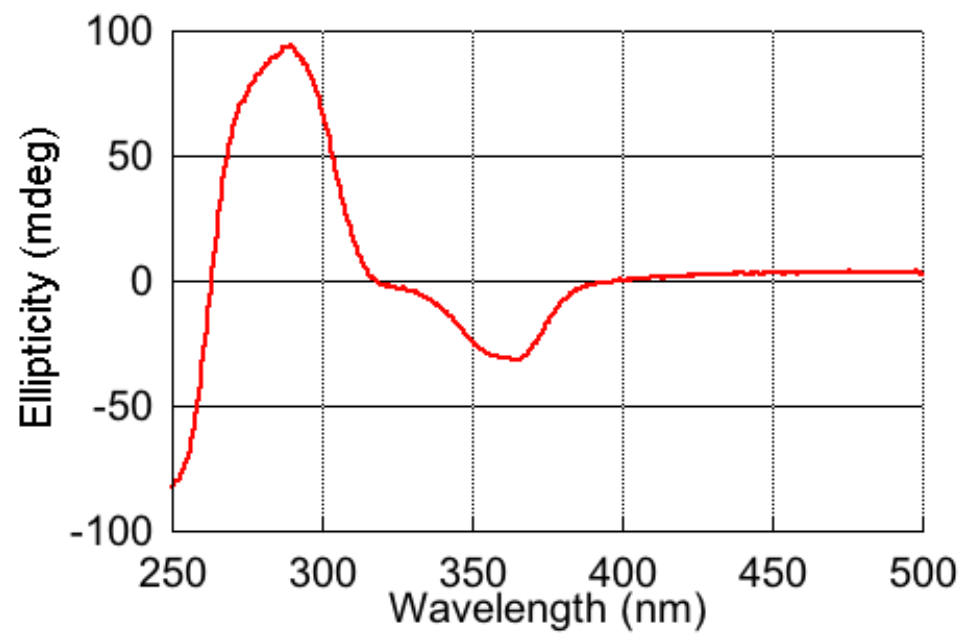

Figure S26. UV-vis absorption spectrum of $\mathbf{P 1 a}(\mathbf{8 0})$ in $\mathrm{CHCl}_{3}$ $\left(3.00 \times 10^{-2} \mathrm{~g} / \mathrm{L}\right.$, light path length $\left.=10 \mathrm{~mm}\right)$. 


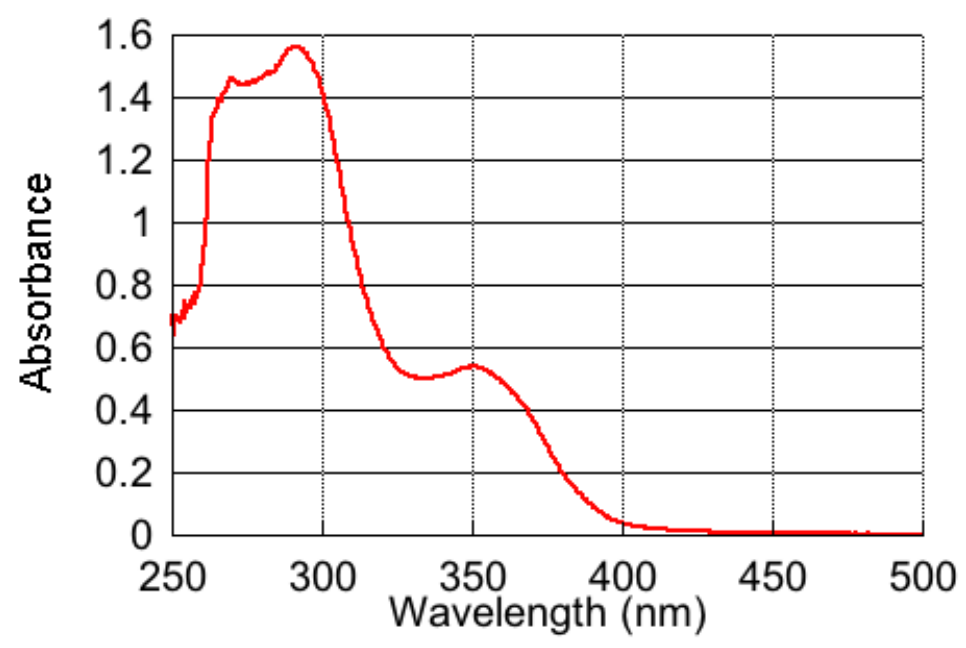

Figure S27. UV-vis absorption spectrum of P1a(80) in 1,1,2-TCE $\left(3.00 \times 10^{-2} \mathrm{~g} / \mathrm{L}\right.$, light path length $\left.=10 \mathrm{~mm}\right)$.

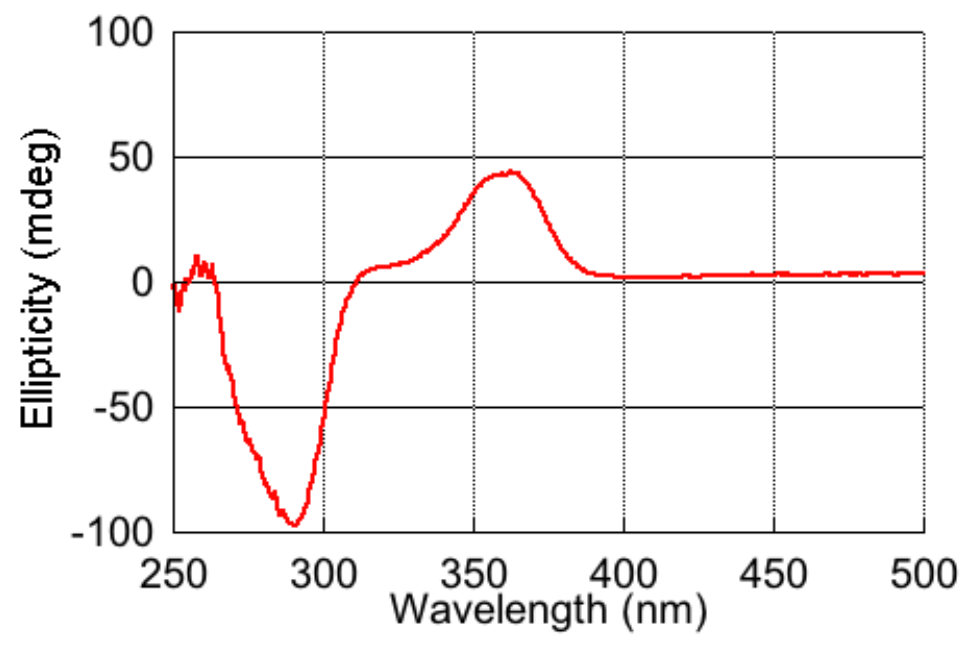

Figure S28. CD spectrum of $\mathbf{P 1 a}(\mathbf{8 0})$ in 1,1,2-TCE $\left(3.00 \times 10^{-2} \mathrm{~g} / \mathrm{L}\right.$, light path length $\left.=10 \mathrm{~mm}\right)$. 


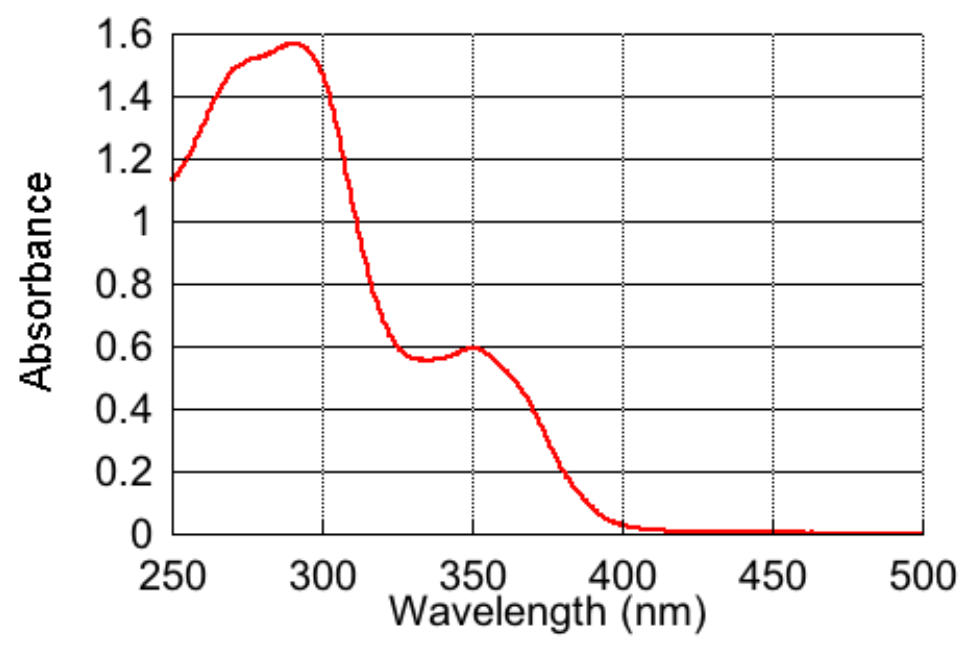

Figure S29. UV-vis absorption spectrum of $\mathbf{P 1 a ( 1 0 0 )}$ in $\mathrm{CHCl}_{3}$

$\left(3.01 \times 10^{-2} \mathrm{~g} / \mathrm{L}\right.$, light path length $\left.=10 \mathrm{~mm}\right)$.

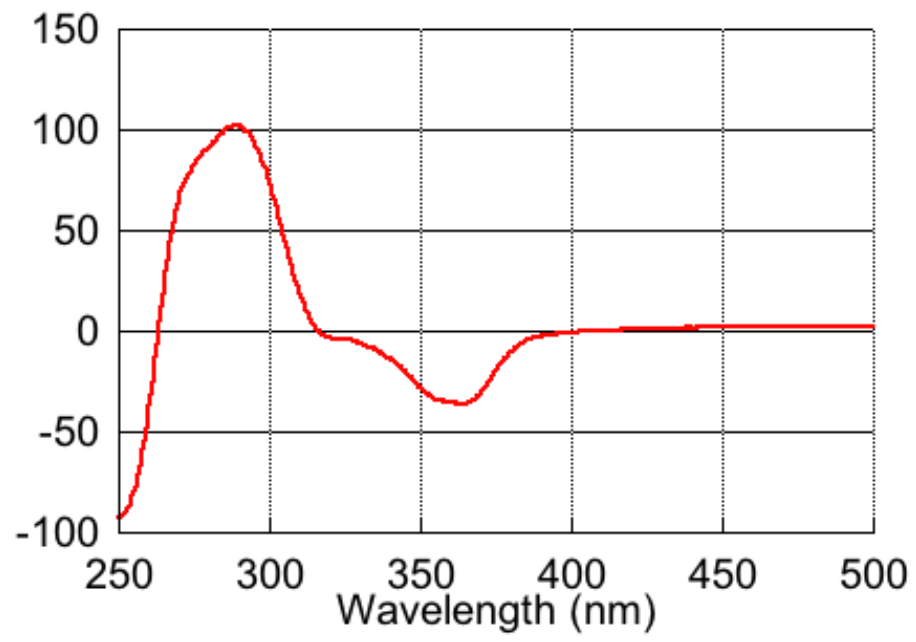

Figure S30. CD spectrum of P1a(100) in $\mathrm{CHCl}_{3}$

$\left(3.01 \times 10^{-2} \mathrm{~g} / \mathrm{L}\right.$, light path length $\left.=10 \mathrm{~mm}\right)$. 


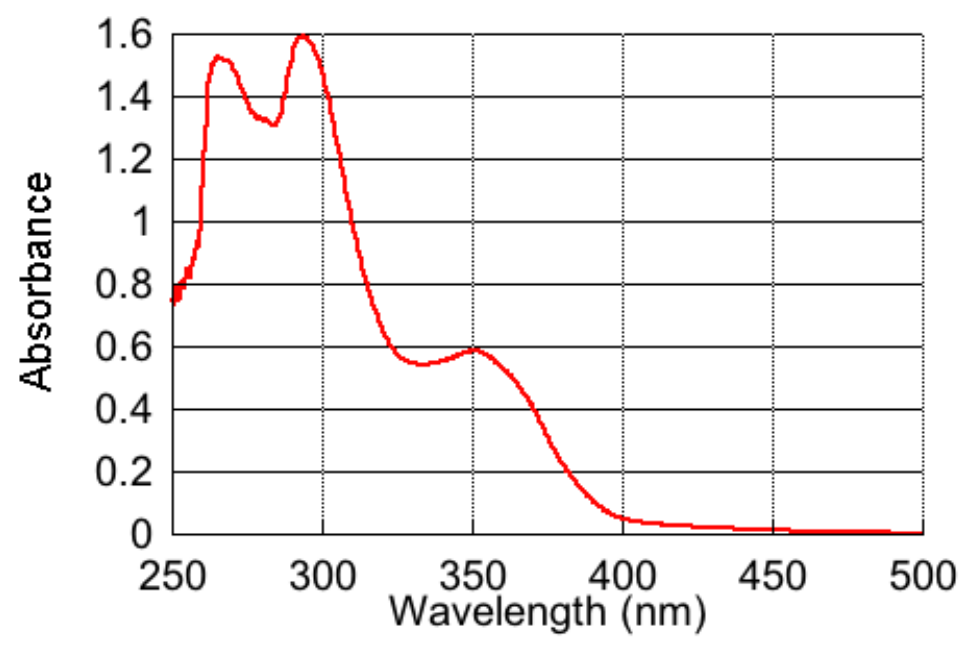

Figure S31. UV-vis absorption spectrum of $\mathbf{P 1 a}(\mathbf{1 0 0})$ in $1,1,2-\mathrm{TCE} / \mathrm{THF}(8 / 2, \mathrm{v} / \mathrm{v})$ $\left(3.01 \times 10^{-2} \mathrm{~g} / \mathrm{L}\right.$, light path length $\left.=10 \mathrm{~mm}\right)$.

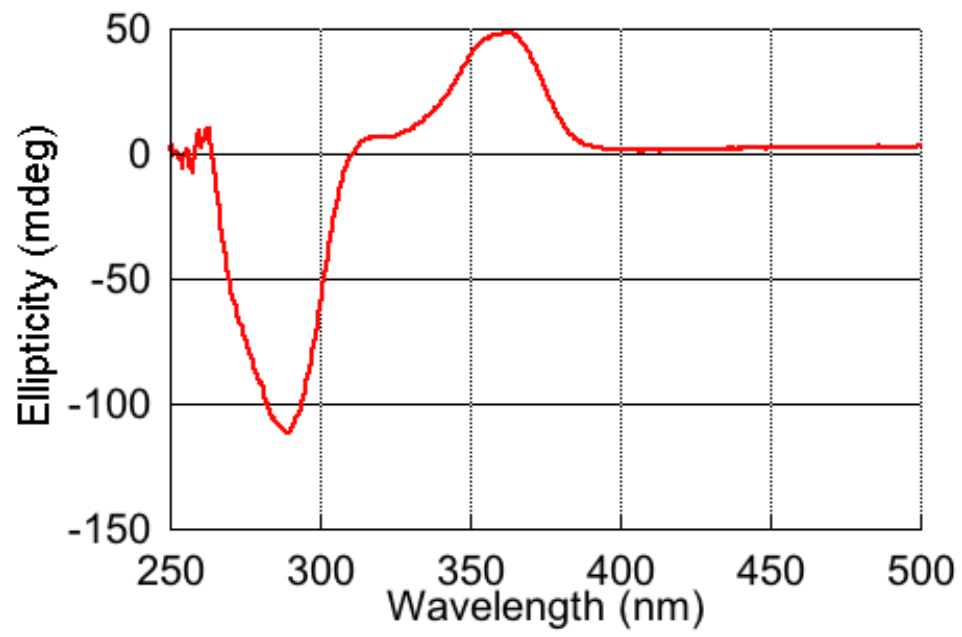

Figure S32. CD spectrum of P1a(100) in 1,1,2-TCE/THF (8/2, v/v) $\left(3.01 \times 10^{-2} \mathrm{~g} / \mathrm{L}\right.$, light path length $\left.=10 \mathrm{~mm}\right)$. 


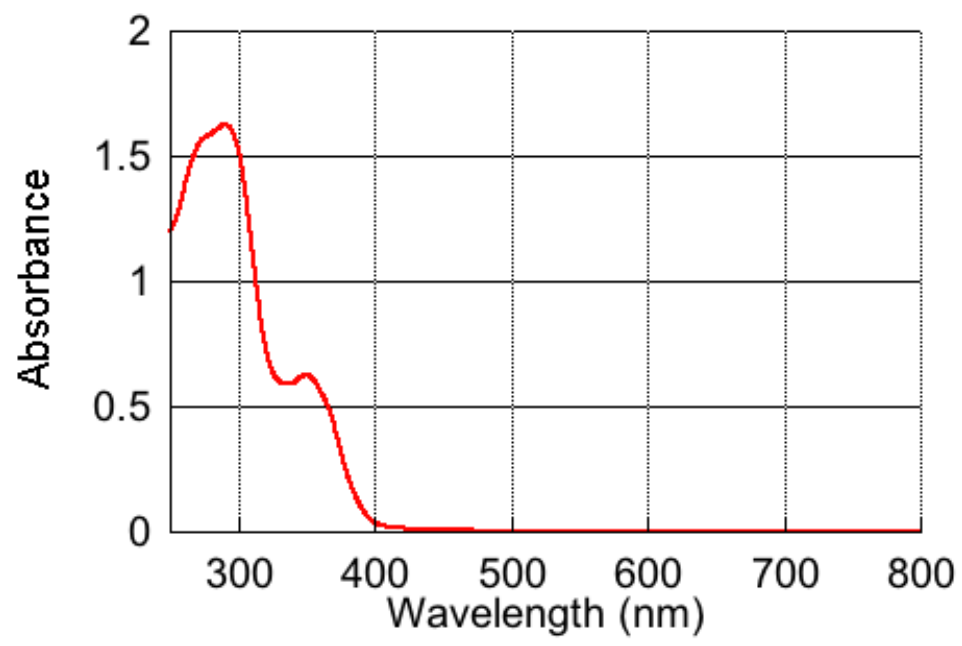

Figure S33. UV-vis absorption spectrum of $\mathbf{P 1 b}(\mathbf{6 0})$ in $\mathrm{CHCl}_{3}$ $\left(2.80 \times 10^{-2} \mathrm{~g} / \mathrm{L}\right.$, light path length $\left.=10 \mathrm{~mm}\right)$.

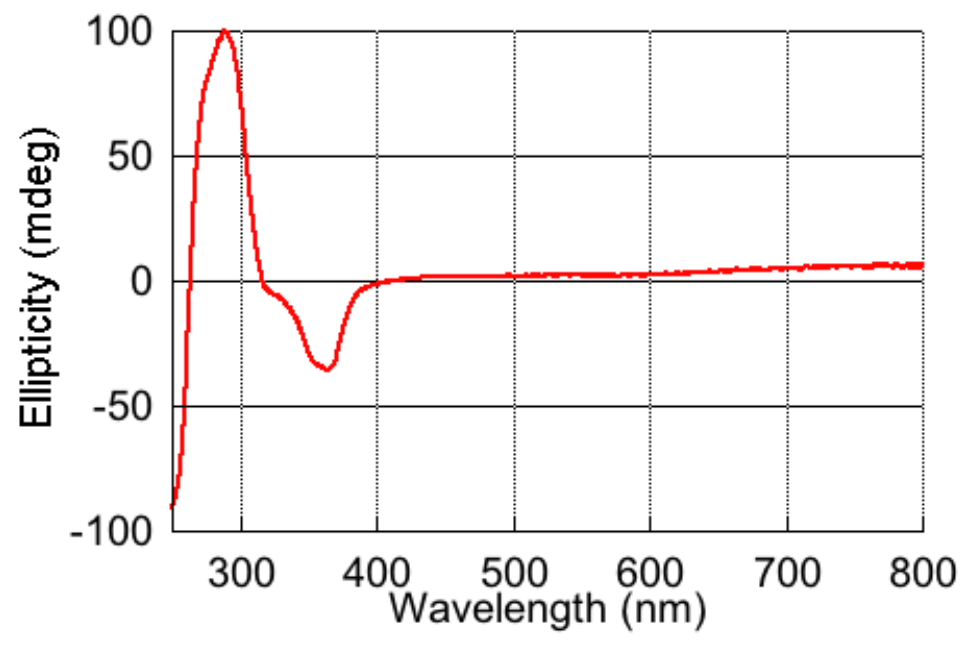

Figure S34. CD spectrum of $\mathbf{P 1 b}(60)$ in $\mathrm{CHCl}_{3}$ $\left(2.80 \times 10^{-2} \mathrm{~g} / \mathrm{L}\right.$, light path length $\left.=10 \mathrm{~mm}\right)$. 


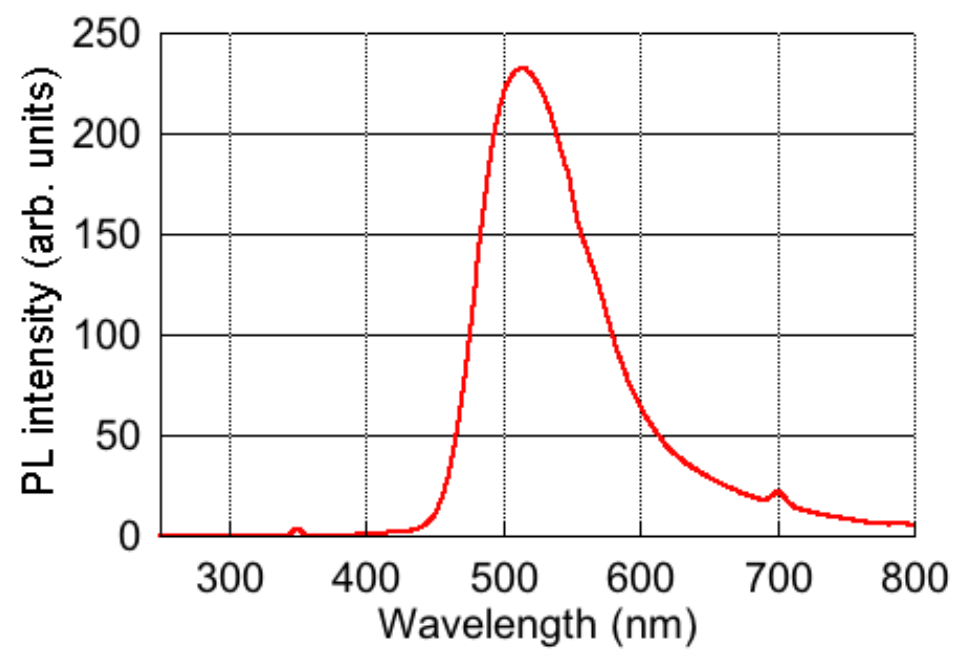

Figure S35. PL spectrum of $\mathbf{P 1 b}(\mathbf{6 0})$ in $\mathrm{CHCl}_{3}$ $\left(2.80 \times 10^{-2} \mathrm{~g} / \mathrm{L}, \lambda_{\mathrm{ex}}=350.0 \mathrm{~nm}\right)$.

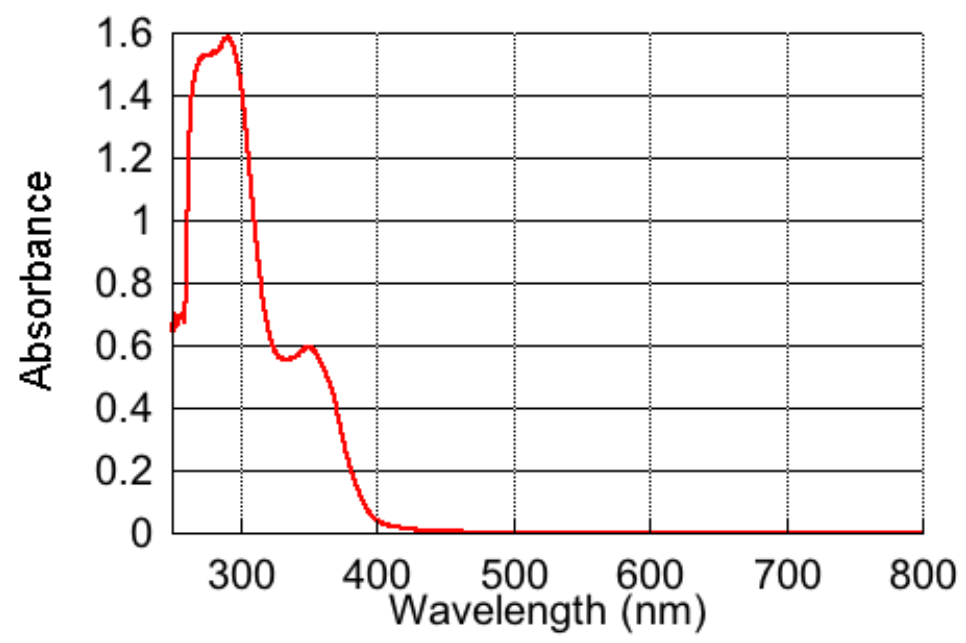

Figure S36. UV-vis absorption spectrum of P1b(60) in 1,1,2-TCE $\left(2.80 \times 10^{-2} \mathrm{~g} / \mathrm{L}\right.$, light path length $\left.=10 \mathrm{~mm}\right)$. 


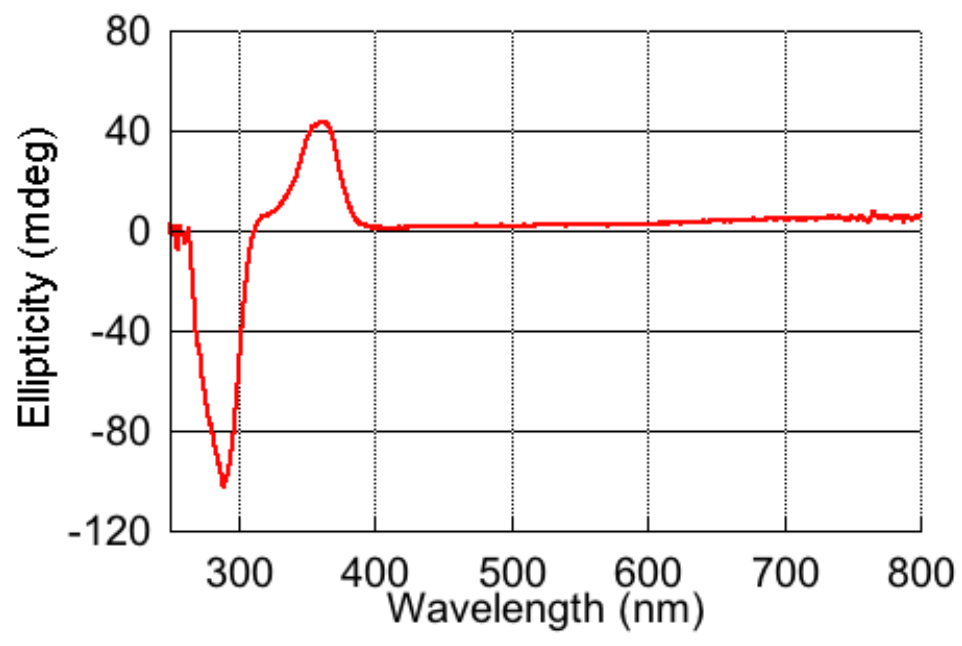

Figure S37. CD spectrum of $\mathbf{P 1 b}(\mathbf{6 0})$ in $1,1,2-\mathrm{TCE}$

$\left(2.80 \times 10^{-2} \mathrm{~g} / \mathrm{L}\right.$, light path length $\left.=10 \mathrm{~mm}\right)$.

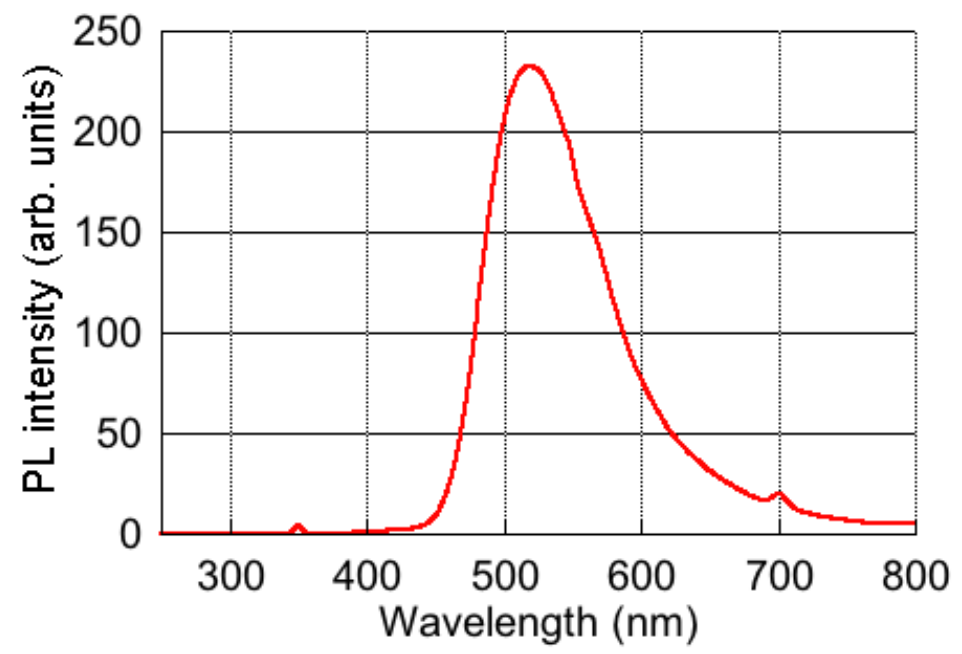

Figure S38. PL spectrum of P1b(60) in 1,1,2-TCE $\left(2.80 \times 10^{-2} \mathrm{~g} / \mathrm{L}, \lambda_{\mathrm{ex}}=350.0 \mathrm{~nm}\right)$. 


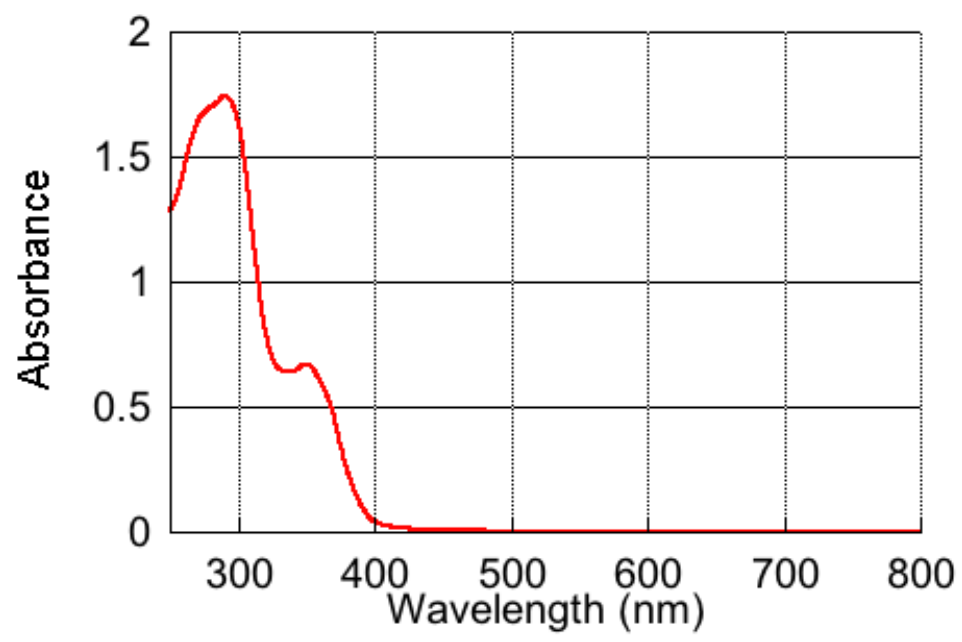

Figure S39. UV-vis absorption spectrum of $\mathbf{P 1 c}(\mathbf{6 0})$ in $\mathrm{CHCl}_{3}$

$\left(3.23 \times 10^{-2} \mathrm{~g} / \mathrm{L}\right.$, light path length $\left.=10 \mathrm{~mm}\right)$.

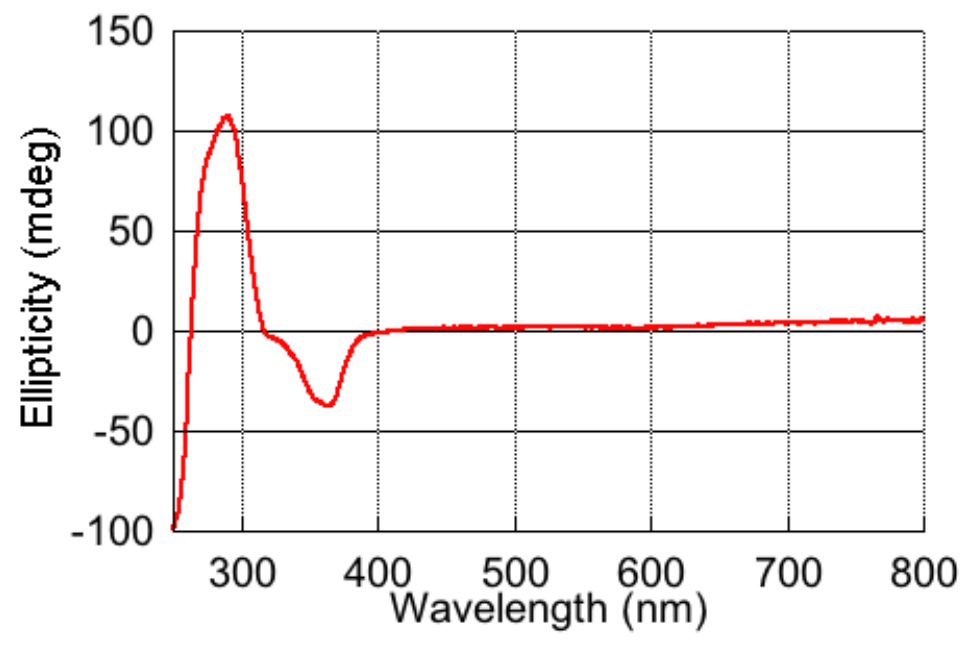

Figure S40. CD spectrum of $\mathbf{P 1 c}(\mathbf{6 0})$ in $\mathrm{CHCl}_{3}$

$\left(3.23 \times 10^{-2} \mathrm{~g} / \mathrm{L}\right.$, light path length $\left.=10 \mathrm{~mm}\right)$. 


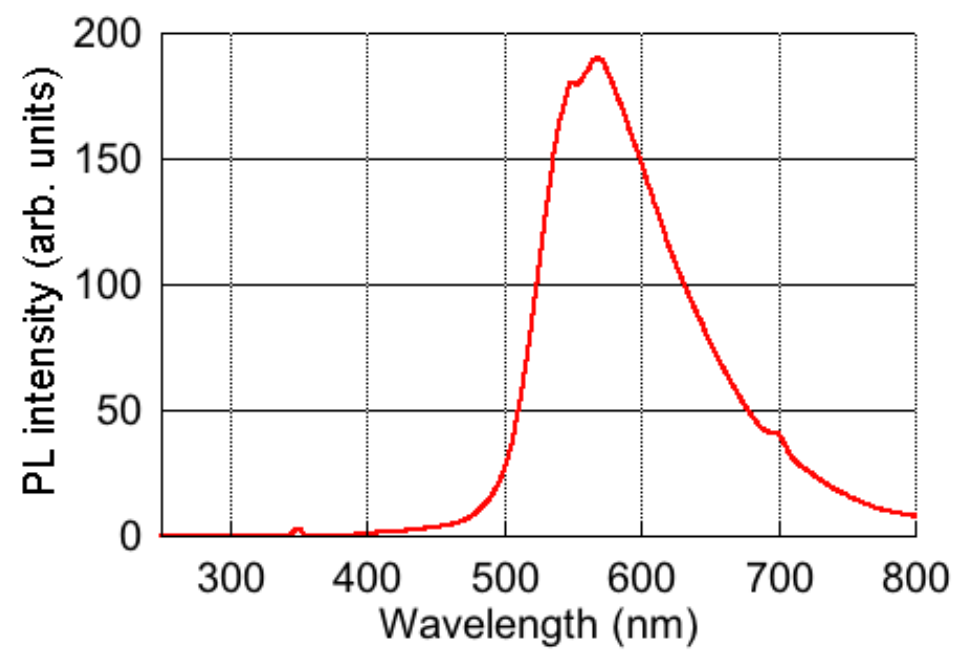

Figure S41. PL spectrum of $\mathbf{P 1 c}(60)$ in $\mathrm{CHCl}_{3}$ $\left(3.23 \times 10^{-2} \mathrm{~g} / \mathrm{L}, \lambda_{\mathrm{ex}}=349.5 \mathrm{~mm}\right)$.

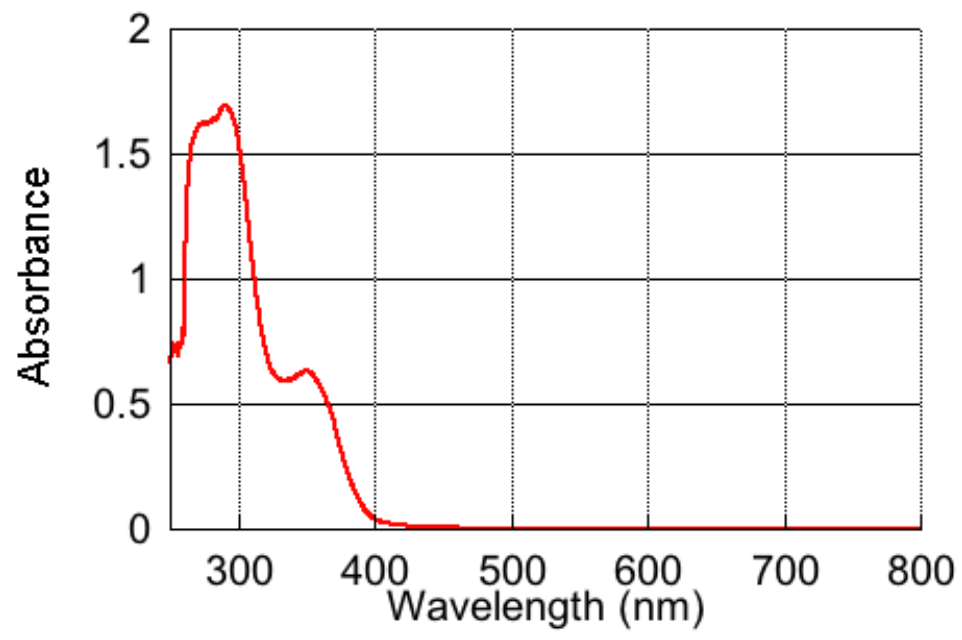

Figure S42. UV-vis absorption spectrum of P1c(60) in 1,1,2-TCE $\left(3.23 \times 10^{-2} \mathrm{~g} / \mathrm{L}\right.$, light path length $\left.=10 \mathrm{~mm}\right)$. 


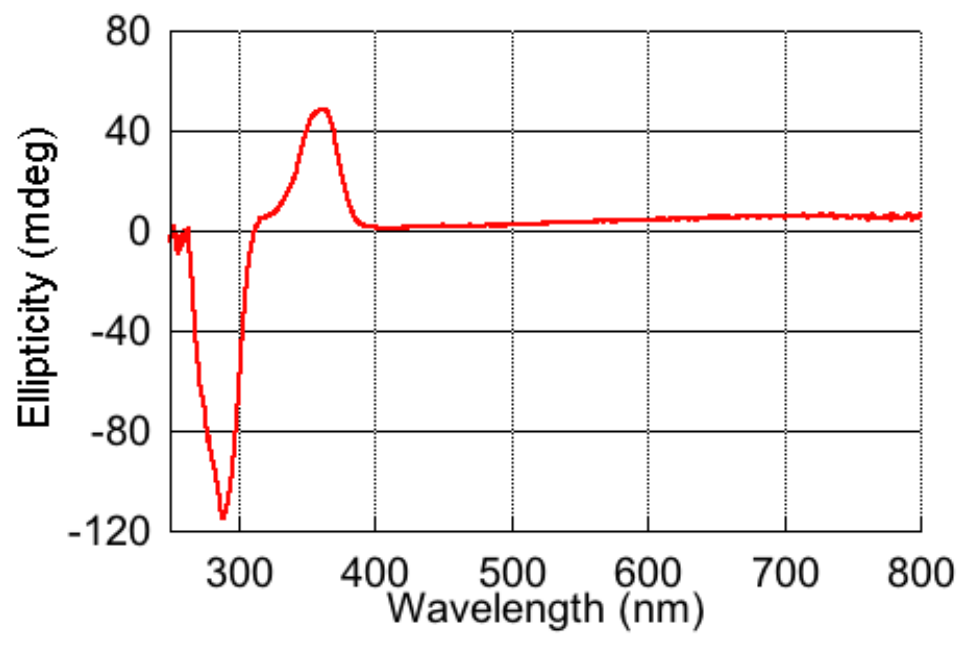

Figure S43. CD spectrum of P1c(60) in 1,1,2-TCE

$\left(3.23 \times 10^{-2} \mathrm{~g} / \mathrm{L}\right.$, light path length $\left.=10 \mathrm{~mm}\right)$.

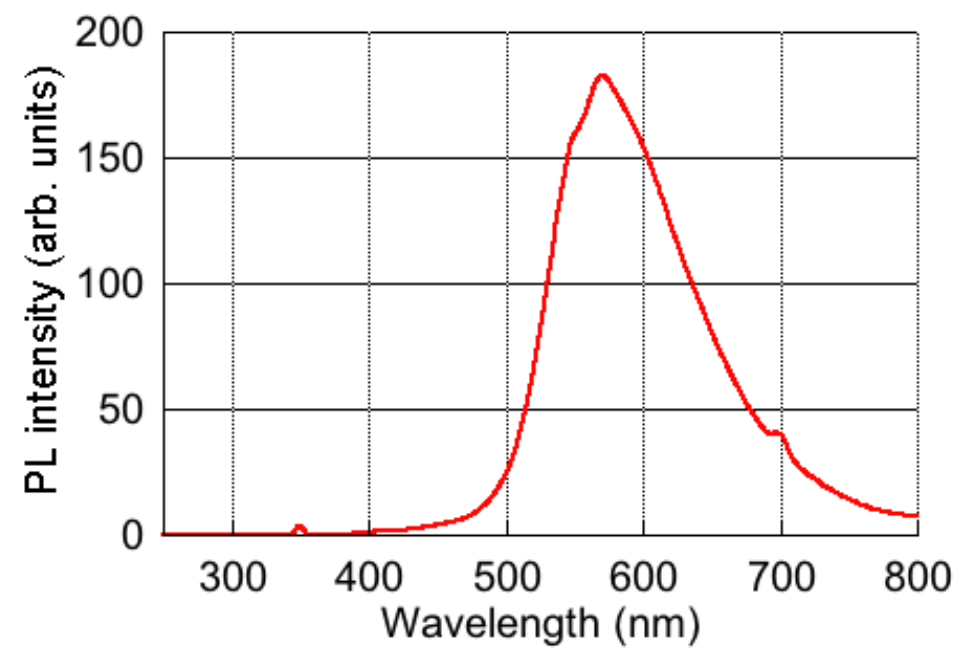

Figure S44. PL spectrum of P1c(60) in 1,1,2-TCE $\left(3.23 \times 10^{-2} \mathrm{~g} / \mathrm{L}, \lambda_{\mathrm{ex}}=349.5 \mathrm{~mm}\right)$. 


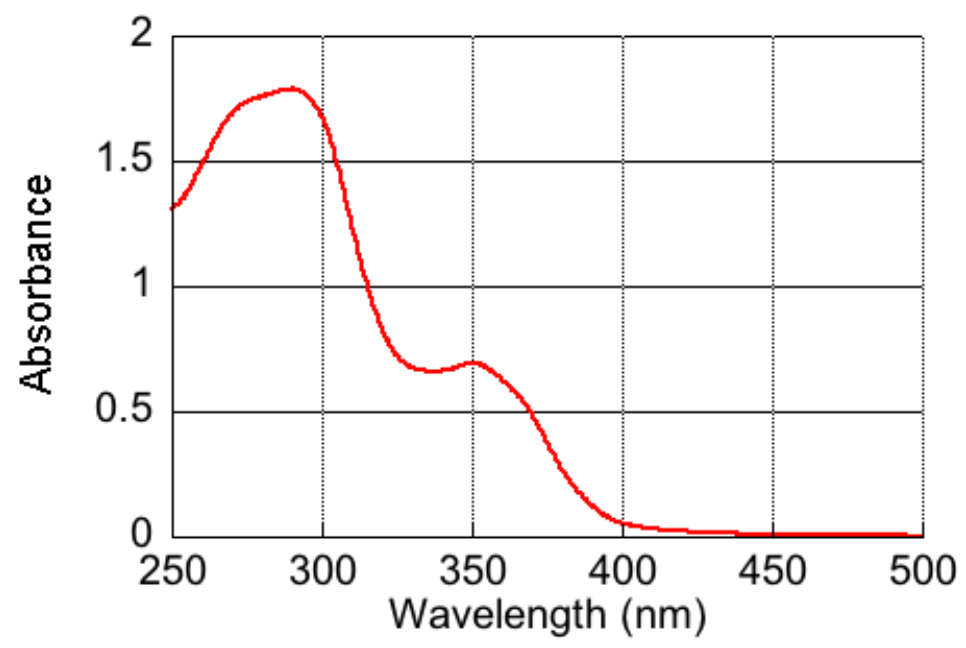

Figure S45. UV-vis spectrum of $\mathbf{P 2 a}(40)$ in $\mathrm{CHCl}_{3}$

$\left(2.99 \times 10^{-2} \mathrm{~g} / \mathrm{L}\right.$, light path length $\left.=10 \mathrm{~mm}\right)$.

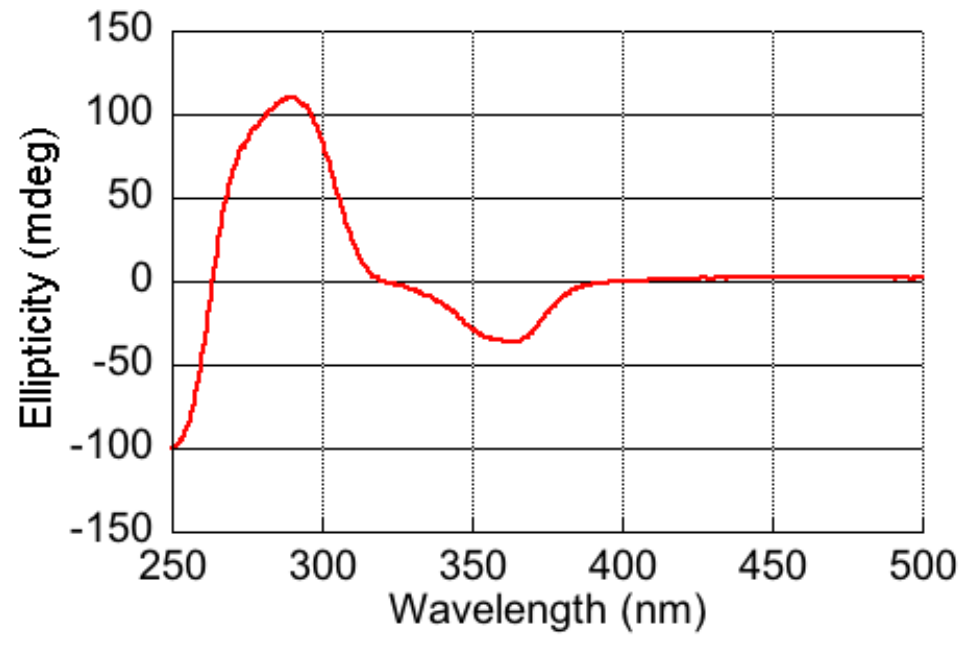

Figure S46. CD spectrum of $\mathbf{P 2 a ( 4 0 )}$ in $\mathrm{CHCl}_{3}$ $\left(2.99 \times 10^{-2} \mathrm{~g} / \mathrm{L}\right.$, light path length $\left.=10 \mathrm{~mm}\right)$. 


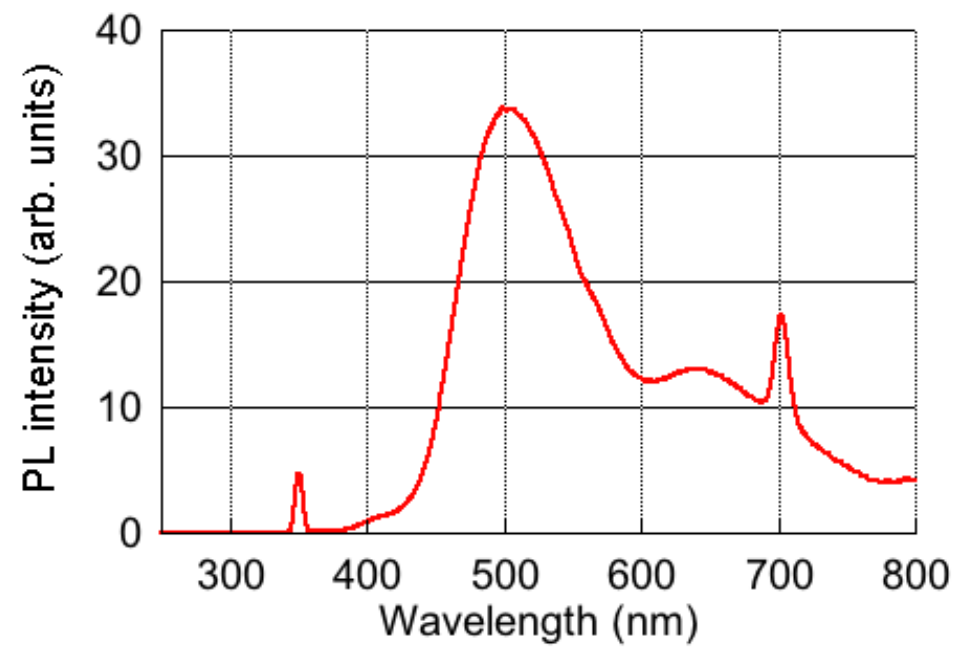

Figure S47. PL spectrum of $\mathbf{P 2 a}(40)$ in $\mathrm{CHCl}_{3}$ $\left(1.75 \times 10^{-2} \mathrm{~g} / \mathrm{L}, \lambda_{\mathrm{ex}}=350.5 \mathrm{~mm}\right)$.

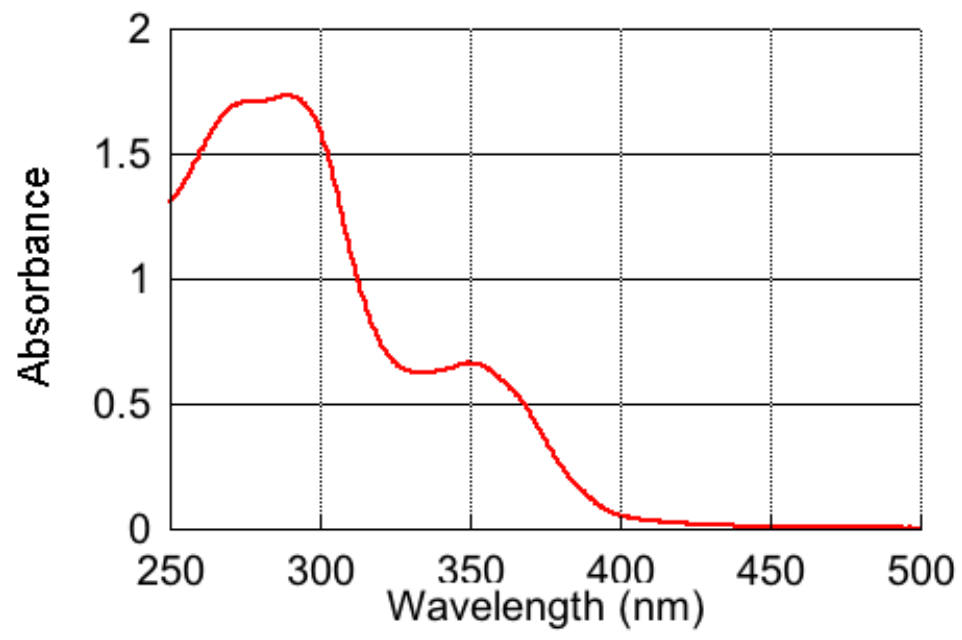

Figure S48. UV-vis absorption spectrum of P2a(40) in 1,1,2-TCE $\left(2.99 \times 10^{-2} \mathrm{~g} / \mathrm{L}\right.$, light path length $\left.=10 \mathrm{~mm}\right)$. 


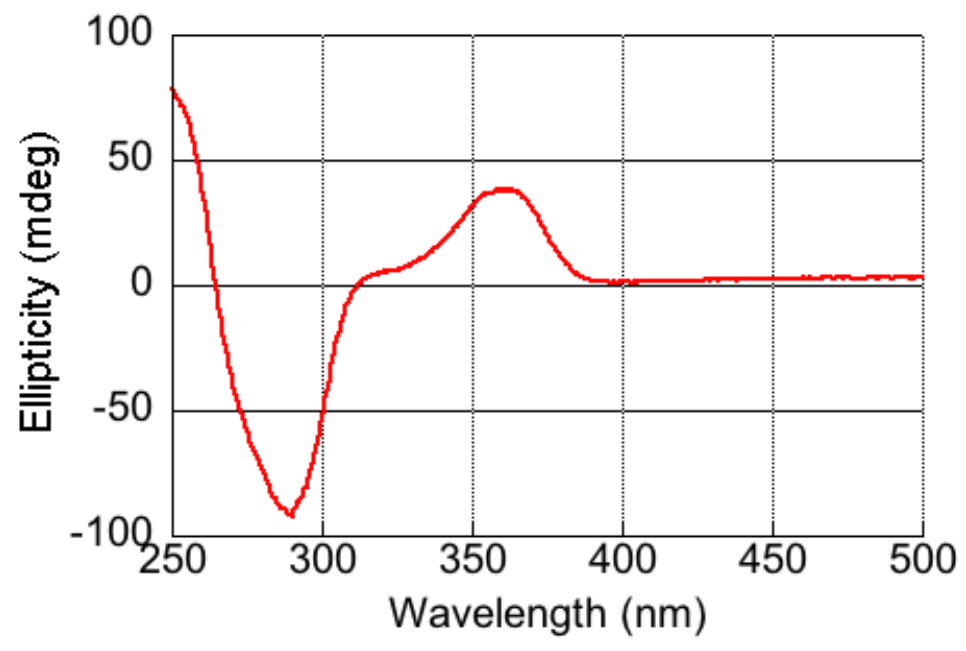

Figure S49. CD spectrum of P2a(40) in 1,1,2-TCE

$\left(2.99 \times 10^{-2} \mathrm{~g} / \mathrm{L}\right.$, light path length $\left.=10 \mathrm{~mm}\right)$.

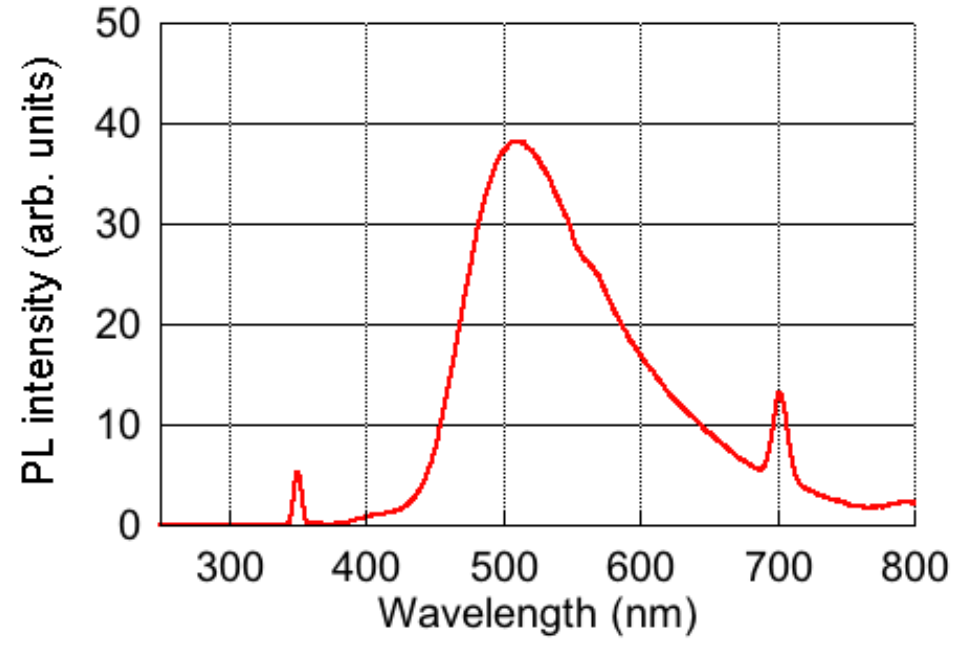

Figure S50. PL spectrum of P2a(40) in 1,1,2-TCE

$$
\left(1.75 \times 10^{-2} \mathrm{~g} / \mathrm{L}, \lambda_{\mathrm{ex}}=350.5 \mathrm{~mm}\right)
$$




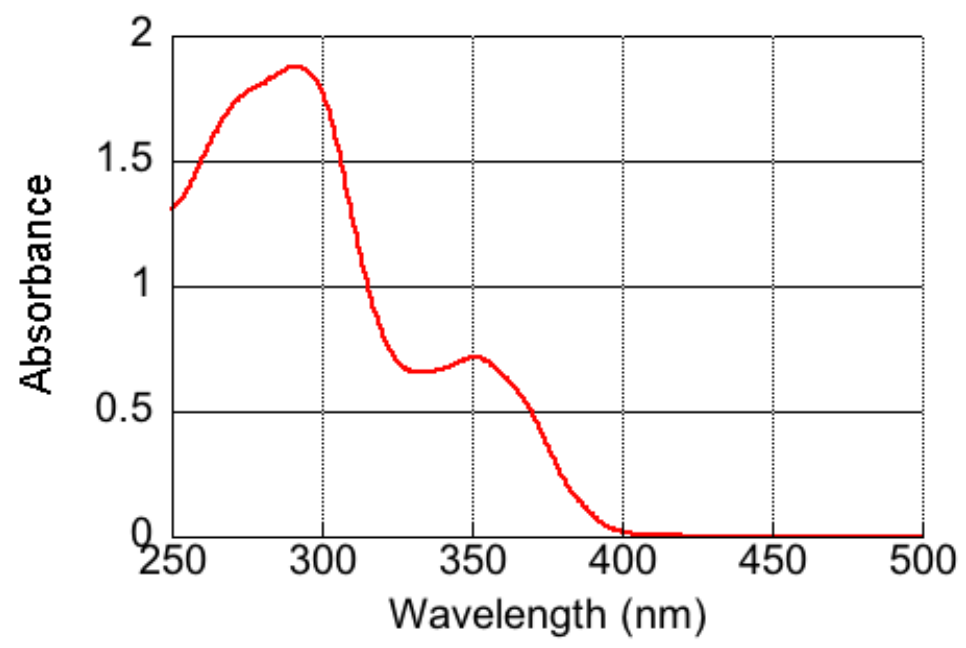

Figure S51. UV-vis absorption spectrum of $\mathbf{P 4}$ in $\mathrm{CHCl}_{3}$

$\left(3.12 \times 10^{-2} \mathrm{~g} / \mathrm{L}\right.$, light path length $\left.=10 \mathrm{~mm}\right)$.

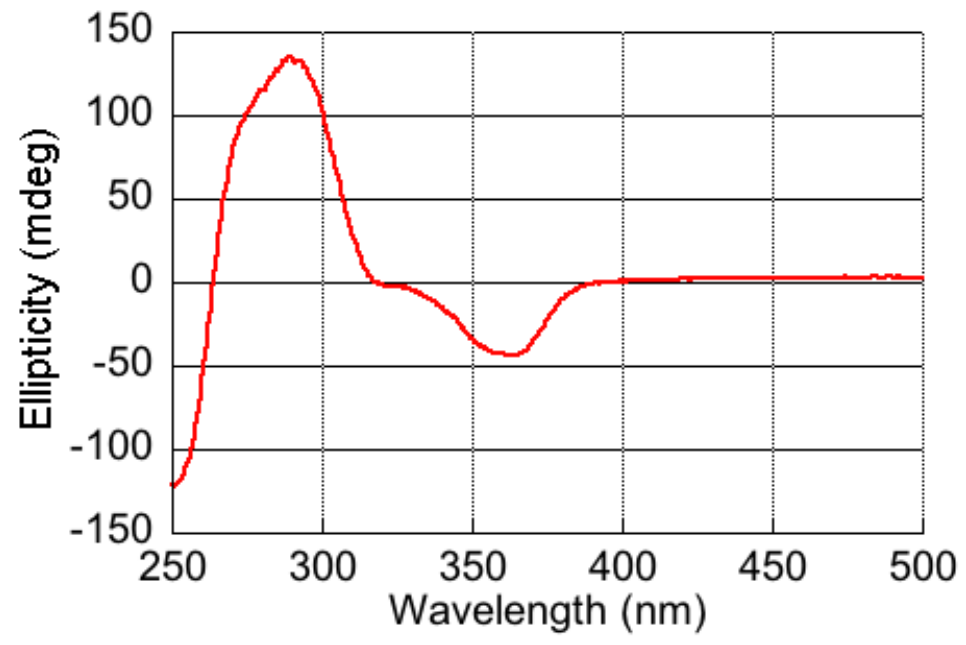

Figure S52. CD spectrum of $\mathbf{P 4}$ in $\mathrm{CHCl}_{3}$

$\left(3.12 \times 10^{-2} \mathrm{~g} / \mathrm{L}\right.$, light path length $\left.=10 \mathrm{~mm}\right)$. 


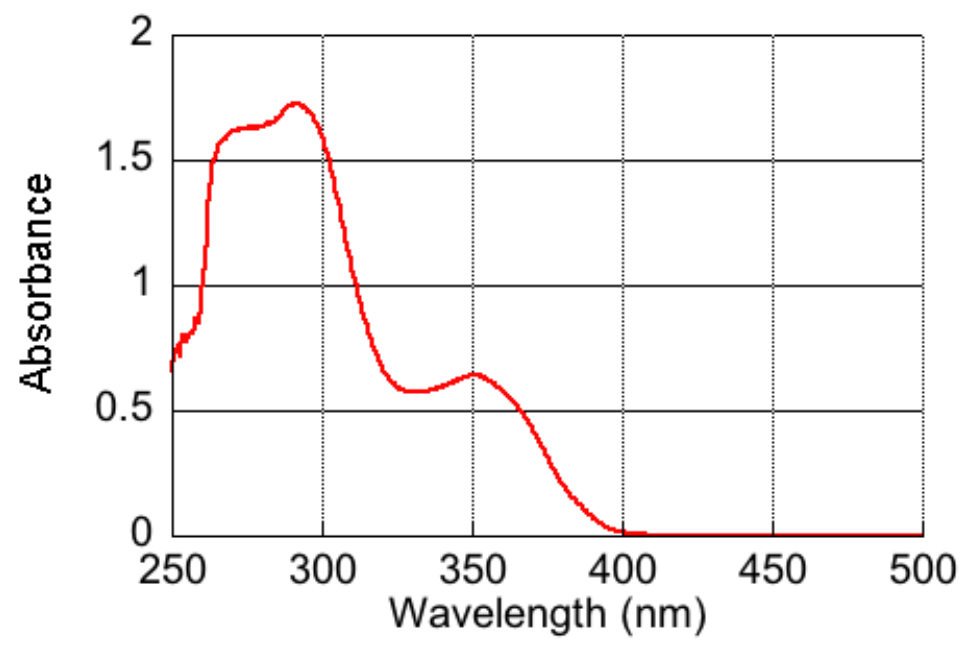

Figure S53. UV-vis absorption spectrum of $\mathbf{P 4}$ in 1,1,2-TCE

$\left(3.12 \times 10^{-2} \mathrm{~g} / \mathrm{L}\right.$, light path length $\left.=10 \mathrm{~mm}\right)$.

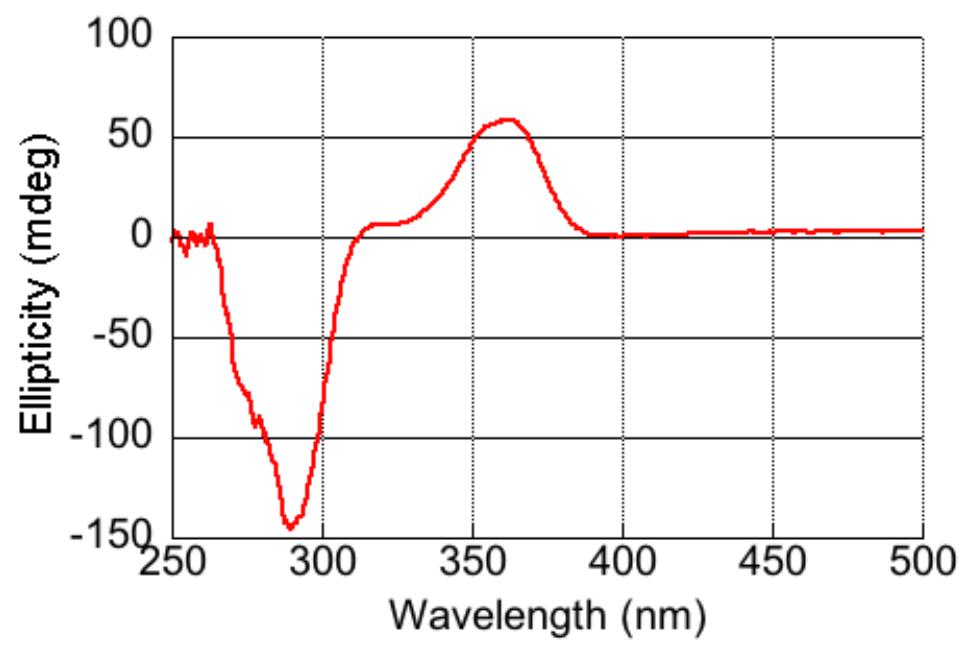

Figure S54. CD spectrum of P4 in 1,1,2-TCE

$\left(3.12 \times 10^{-2} \mathrm{~g} / \mathrm{L}\right.$, light path length $\left.=10 \mathrm{~mm}\right)$. 


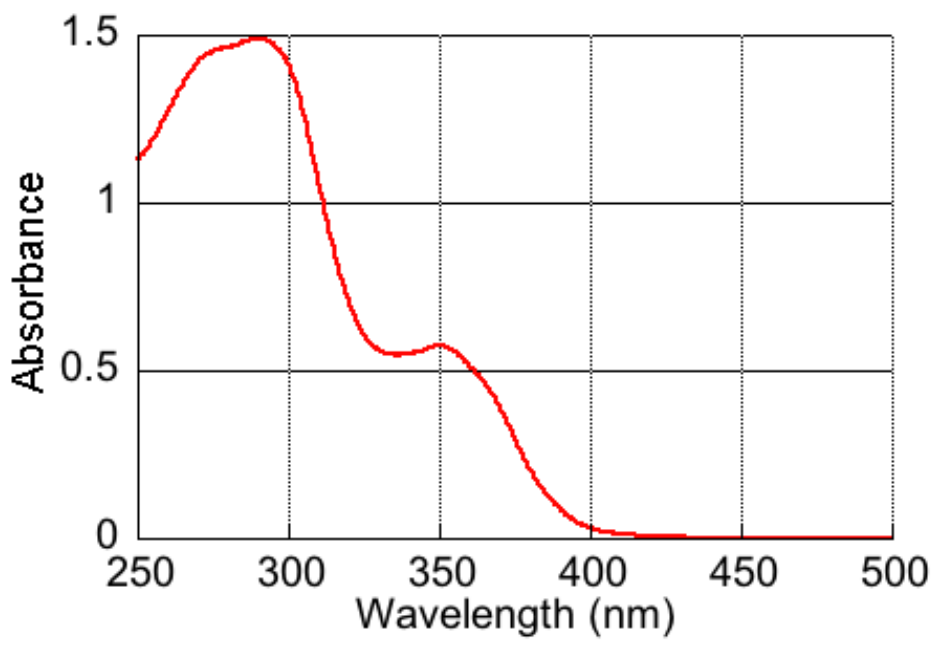

Figure S55. UV-vis absorption spectrum of $\mathbf{P 5}$ in $\mathrm{CHCl}_{3}$

$\left(2.43 \times 10^{-2} \mathrm{~g} / \mathrm{L}\right.$, light path length $\left.=10 \mathrm{~mm}\right)$.

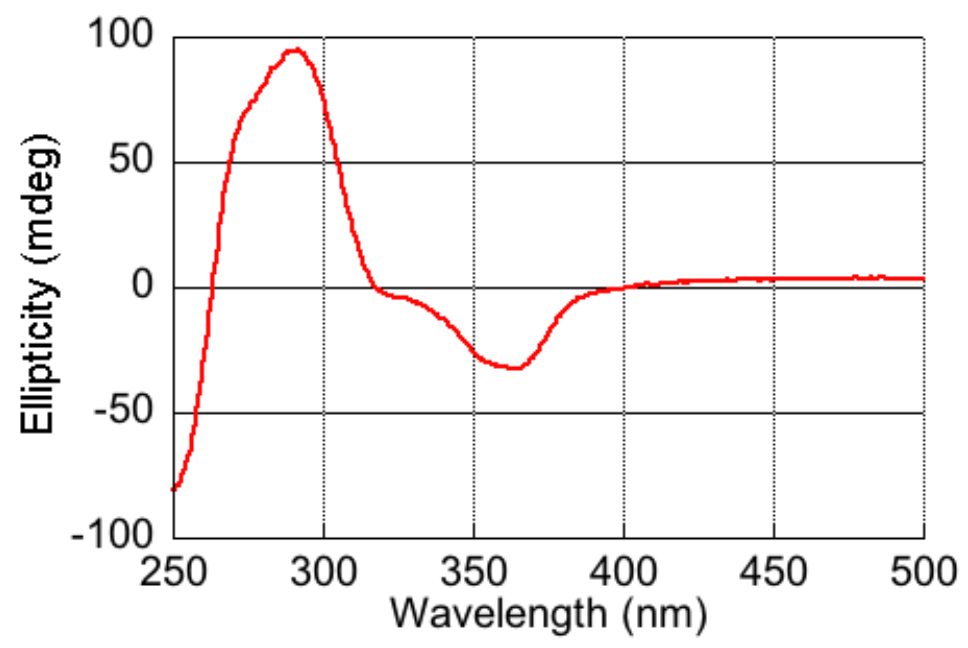

Figure S56. CD spectrum of $\mathbf{P 5}$ in $\mathrm{CHCl}_{3}$

$\left(2.43 \times 10^{-2} \mathrm{~g} / \mathrm{L}\right.$, light path length $\left.=10 \mathrm{~mm}\right)$. 


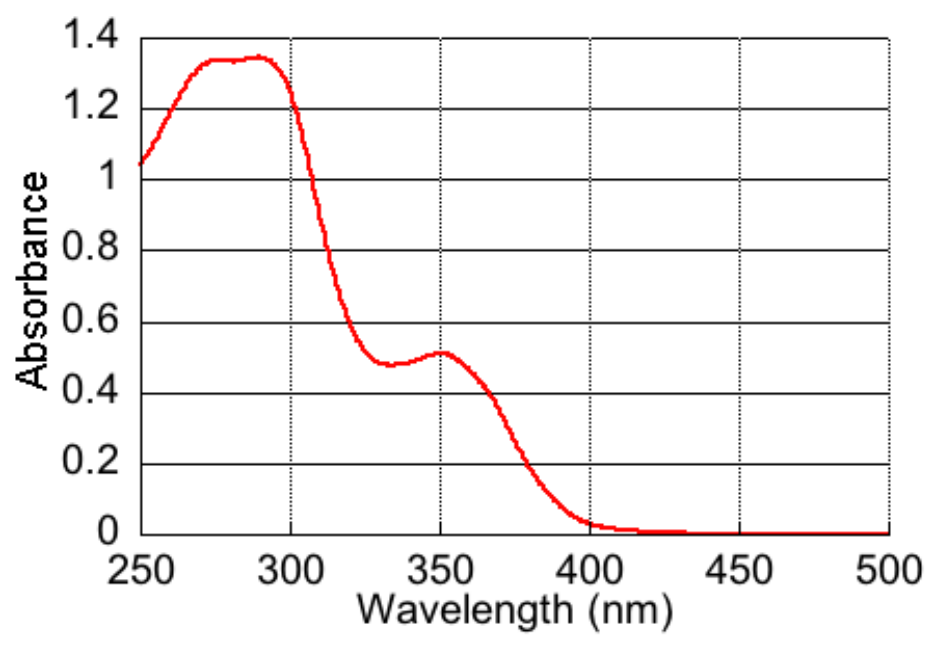

Figure S57. UV-vis spectrum of $\mathbf{P 5}$ in 1,1,2-TCE $\left(2.43 \times 10^{-2} \mathrm{~g} / \mathrm{L}\right.$, light path length $\left.=10 \mathrm{~mm}\right)$.

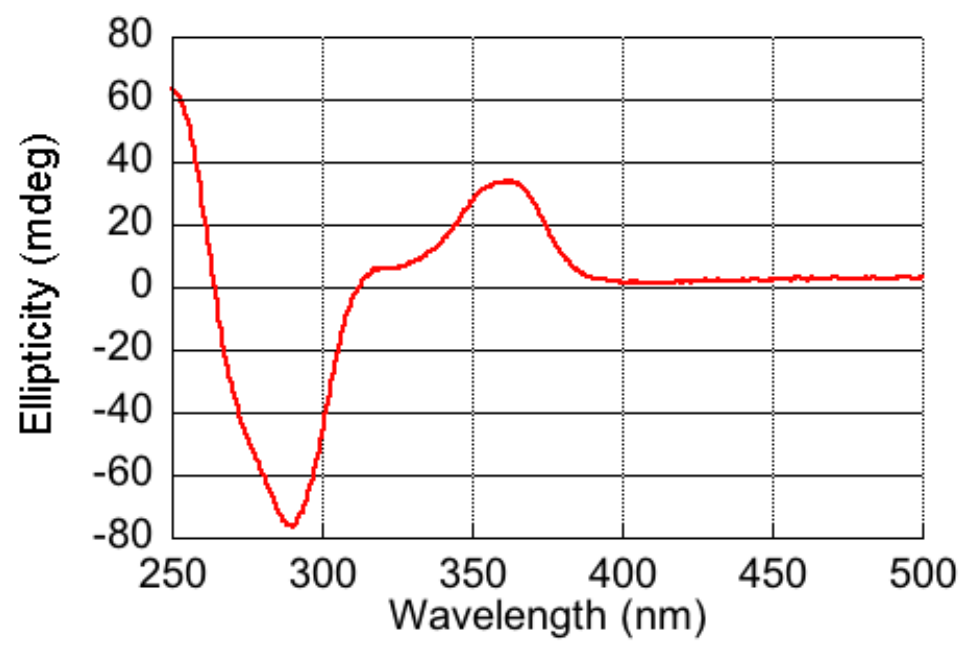

Figure S58. CD spectrum of P5 in 1,1,2-TCE $\left(2.43 \times 10^{-2} \mathrm{~g} / \mathrm{L}\right.$, light path length $\left.=10 \mathrm{~mm}\right)$. 


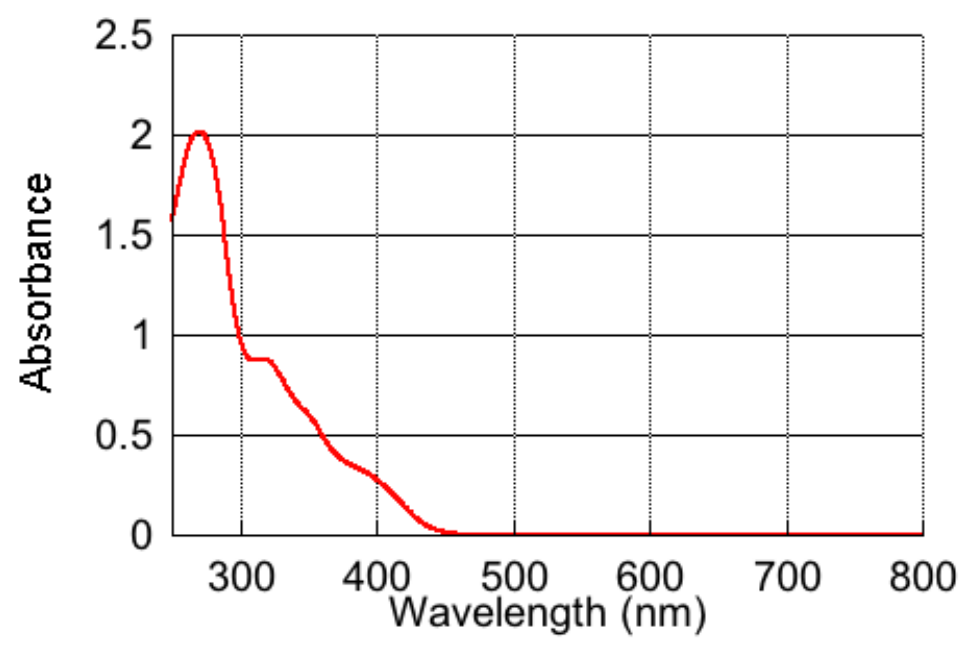

Figure S59. UV-vis absorption spectrum of $\mathbf{2 a}$ in $\mathrm{CHCl}_{3}$

$\left(1.99 \times 10^{-2} \mathrm{~g} / \mathrm{L}\right.$, light path length $\left.=10 \mathrm{~mm}\right)$.

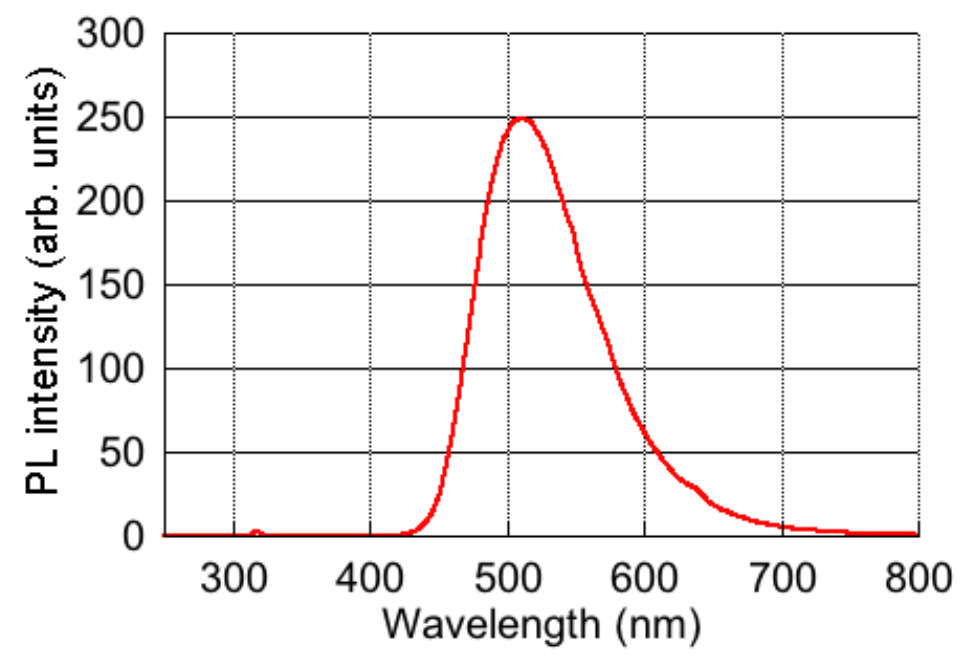

Figure S60. PL spectrum of $\mathbf{2 a}$ in $\mathrm{CHCl}_{3}$

$$
\left(1.99 \times 10^{-2} \mathrm{~g} / \mathrm{L}, \lambda_{\mathrm{ex}}=317.0 \mathrm{~mm}\right) \text {. }
$$




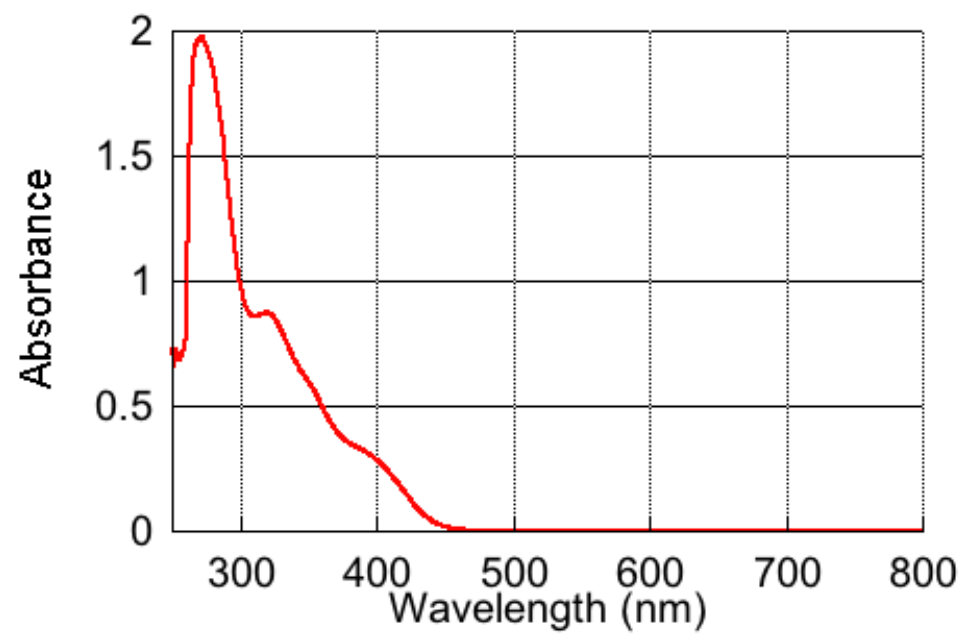

Figure S61. UV-vis absorption spectrum of $\mathbf{2 a}$ in 1,1,2-TCE $\left(1.99 \times 10^{-2} \mathrm{~g} / \mathrm{L}\right.$, light path length $\left.=10 \mathrm{~mm}\right)$.

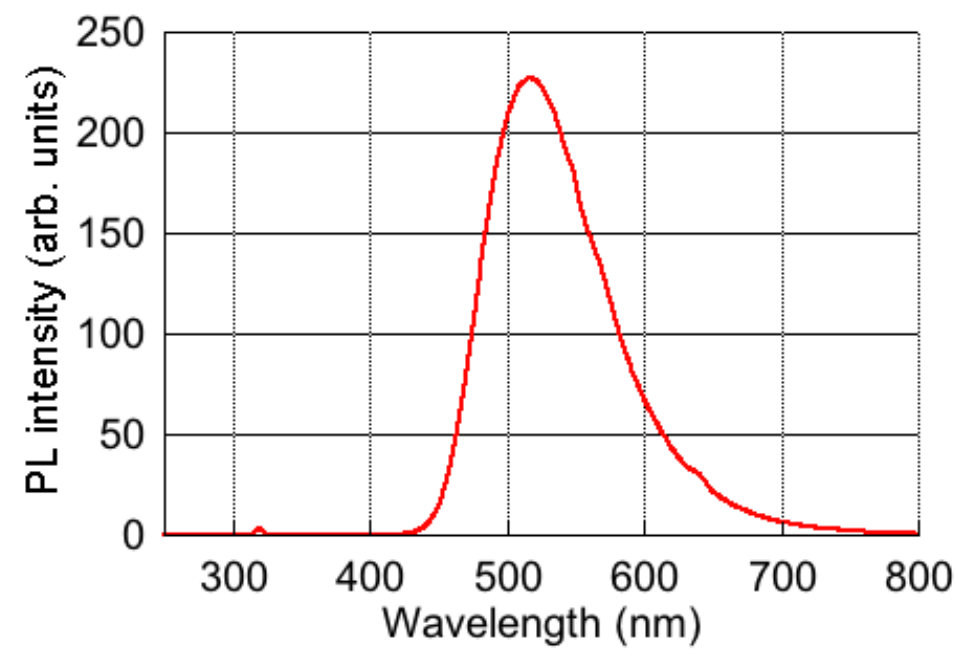

Figure S62. PL spectrum of 2a in 1,1,2-TCE $\left(1.99 \times 10^{-2} \mathrm{~g} / \mathrm{L}, \lambda_{\mathrm{ex}}=319.0 \mathrm{~mm}\right)$. 


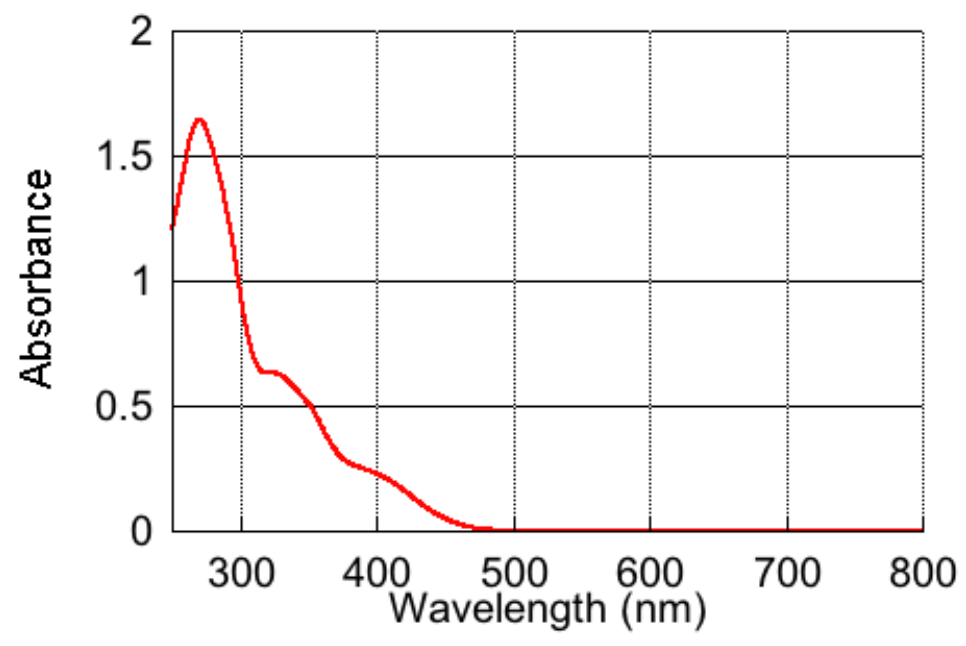

Figure S63. UV-vis absorption spectrum of $\mathbf{2 b}$ in $\mathrm{CHCl}_{3}$

$\left(1.93 \times 10^{-2} \mathrm{~g} / \mathrm{L}\right.$, light path length $\left.=10 \mathrm{~mm}\right)$.

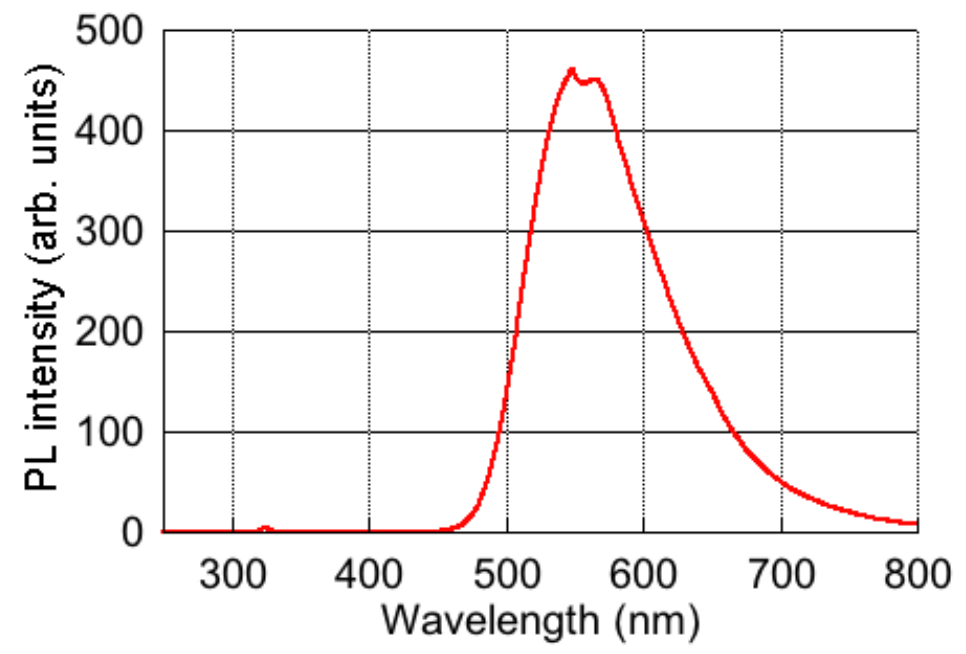

Figure S64. PL spectrum of $\mathbf{2 b}$ in $\mathrm{CHCl}_{3}$

$$
\left(1.93 \times 10^{-2} \mathrm{~g} / \mathrm{L}, \lambda_{\mathrm{ex}}=324.5 \mathrm{~mm}\right) \text {. }
$$




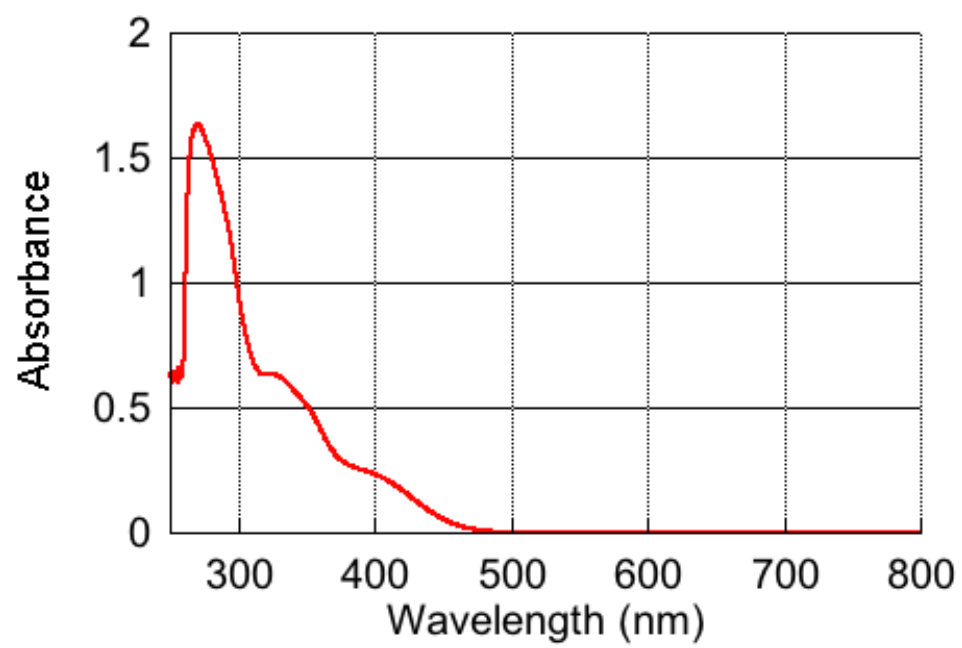

Figure S65. UV-vis absorption spectrum of $\mathbf{2 b}$ in 1,1,2-TCE $\left(1.93 \times 10^{-2} \mathrm{~g} / \mathrm{L}\right.$, light path length $\left.=10 \mathrm{~mm}\right)$.

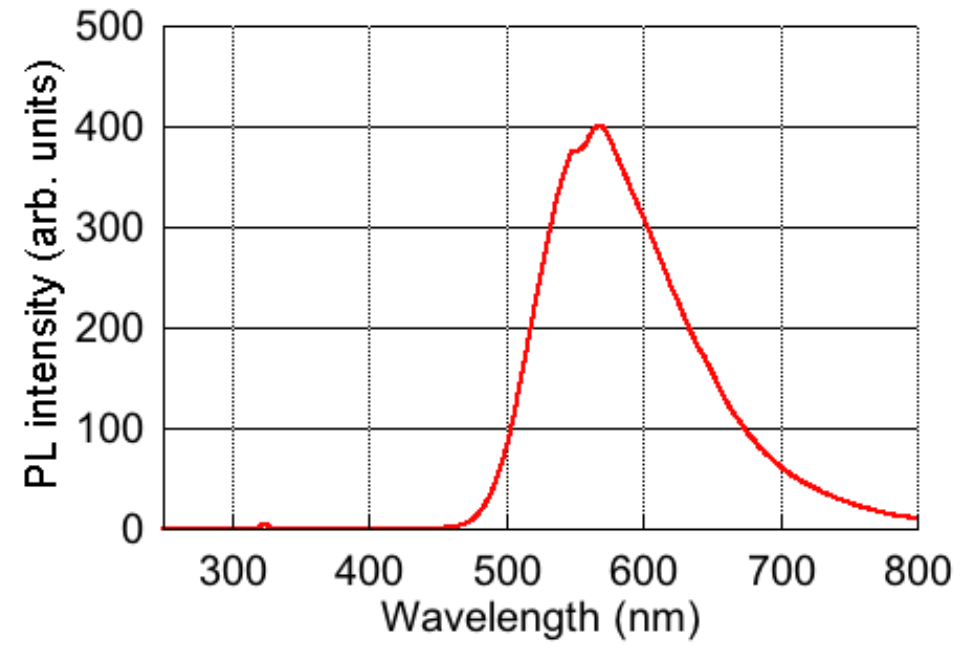

Figure S66. PL spectrum of $\mathbf{2 b}$ in 1,1,2-TCE $\left(1.93 \times 10^{-2} \mathrm{~g} / \mathrm{L}, \lambda_{\mathrm{ex}}=324.5 \mathrm{~mm}\right)$. 


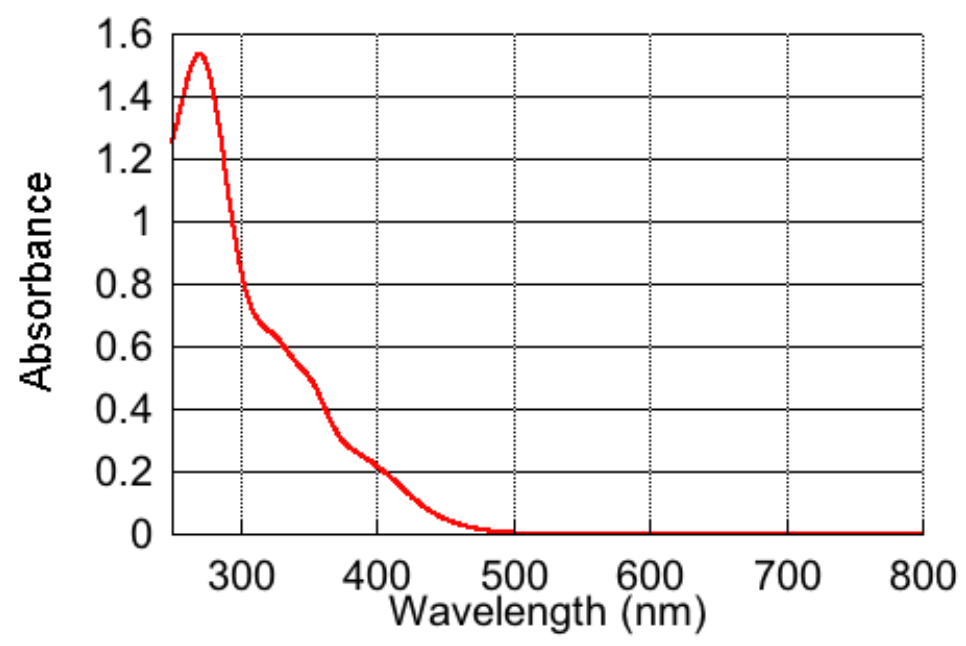

Figure S67. UV-vis absorption spectrum of $2 \mathrm{c}$ in $\mathrm{CHCl}_{3}$ $\left(1.89 \times 10^{-2} \mathrm{~g} / \mathrm{L}\right.$, light path length $\left.=10 \mathrm{~mm}\right)$.

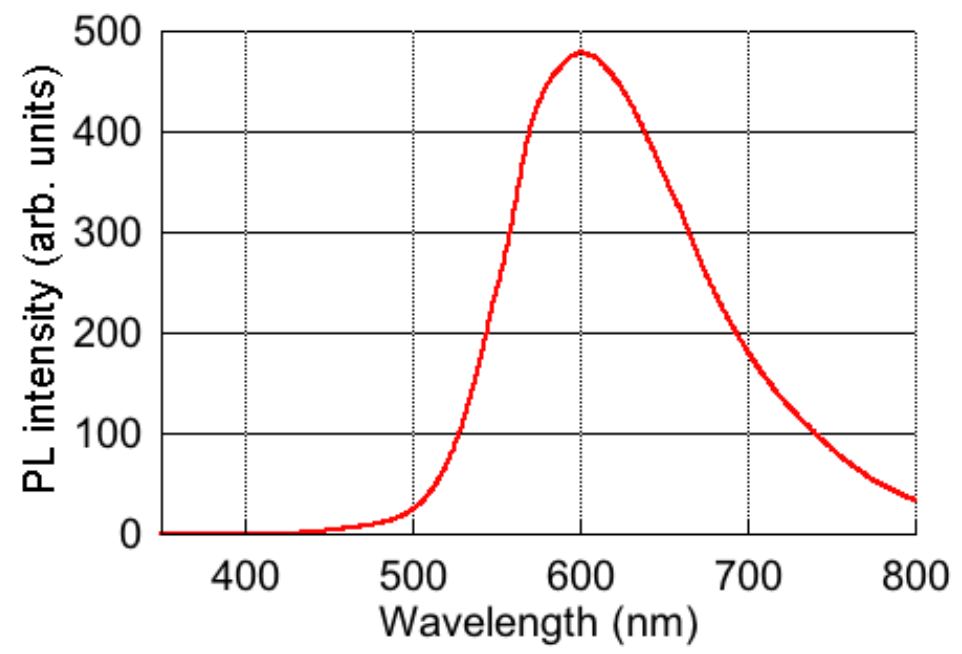

Figure S68. PL spectrum of $2 \mathbf{c}$ in $\mathrm{CHCl}_{3}$ $\left(1.89 \times 10^{-2} \mathrm{~g} / \mathrm{L}, \lambda_{\mathrm{ex}}=329.0 \mathrm{~mm}\right)$. 


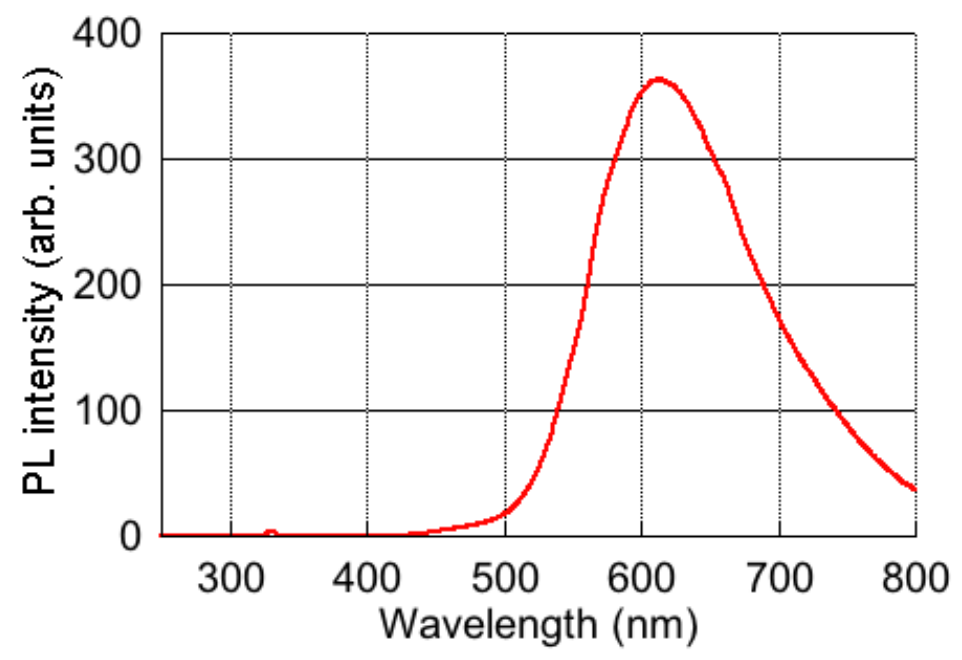

Figure S69. PL spectrum of $\mathbf{2 c}$ in 1,1,2-TCE $\left(1.89 \times 10^{-2} \mathrm{~g} / \mathrm{L}, \lambda_{\mathrm{ex}}=330.0 \mathrm{~mm}\right)$.

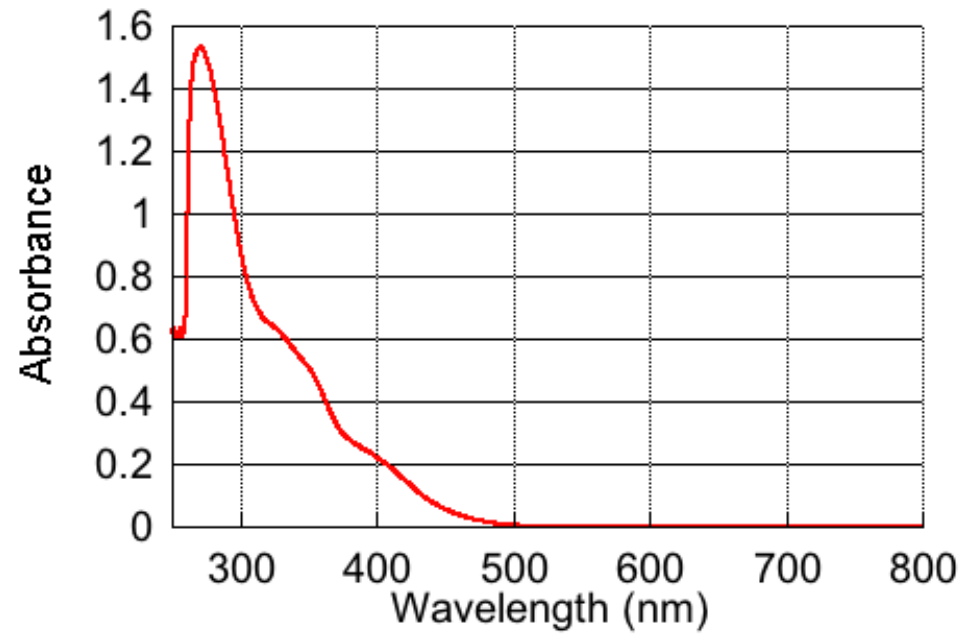

Figure S70. UV-vis absorption spectrum of $\mathbf{2 c}$ in 1,1,2-TCE $\left(1.89 \times 10^{-2} \mathrm{~g} / \mathrm{L}\right.$, light path length $\left.=10 \mathrm{~mm}\right)$. 


\section{NMR Spectra of New Compound}

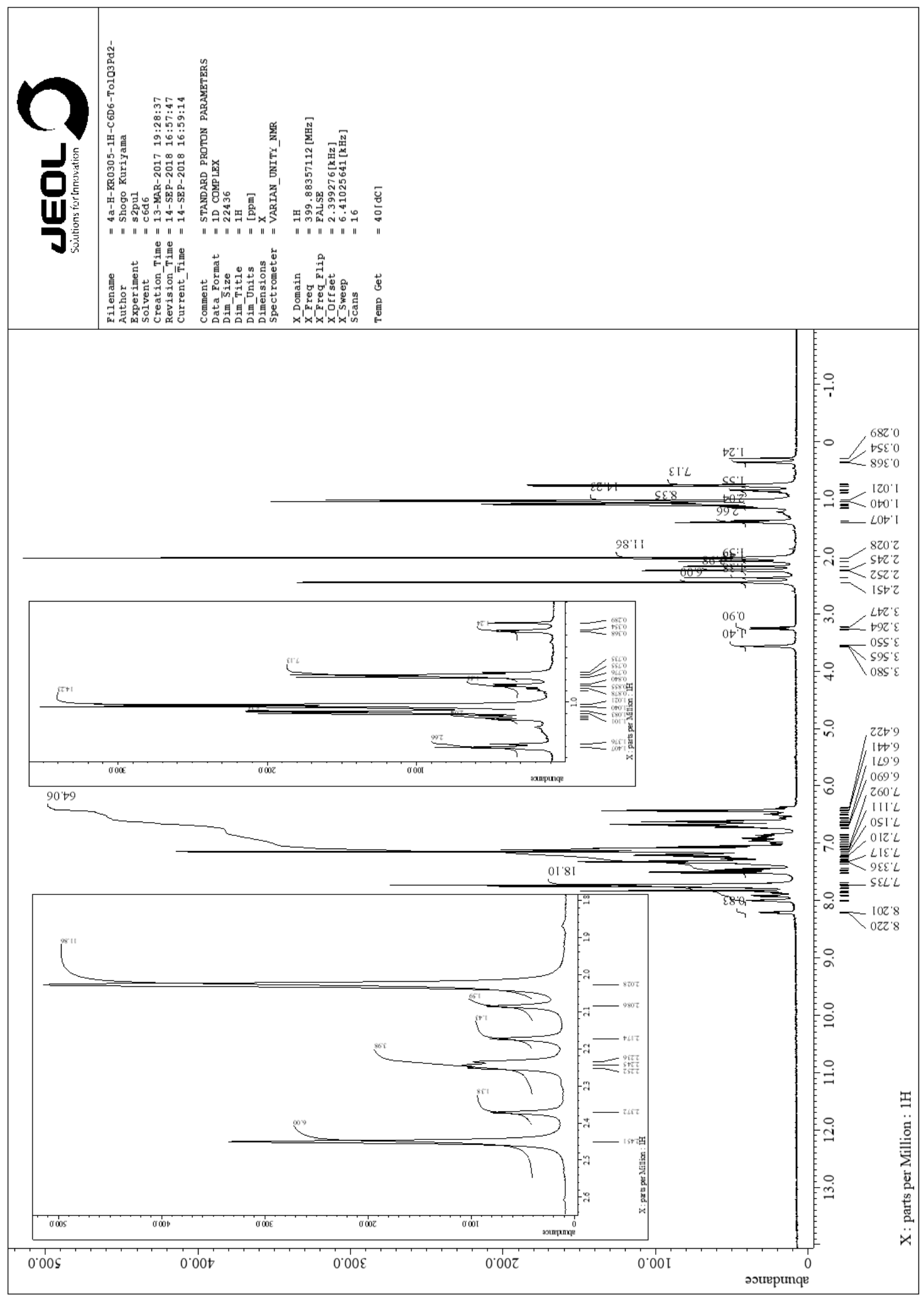

Figure S71. ${ }^{1} \mathrm{H}$ NMR spectrum of $1 \mathrm{a} \cdot 0.3 \mathrm{C}_{4} \mathrm{H}_{8} \mathrm{O} \cdot 0.2 \mathrm{C}_{4} \mathrm{H}_{10} \mathrm{O}$ in $\mathrm{C}_{6} \mathrm{D}_{6}$. 


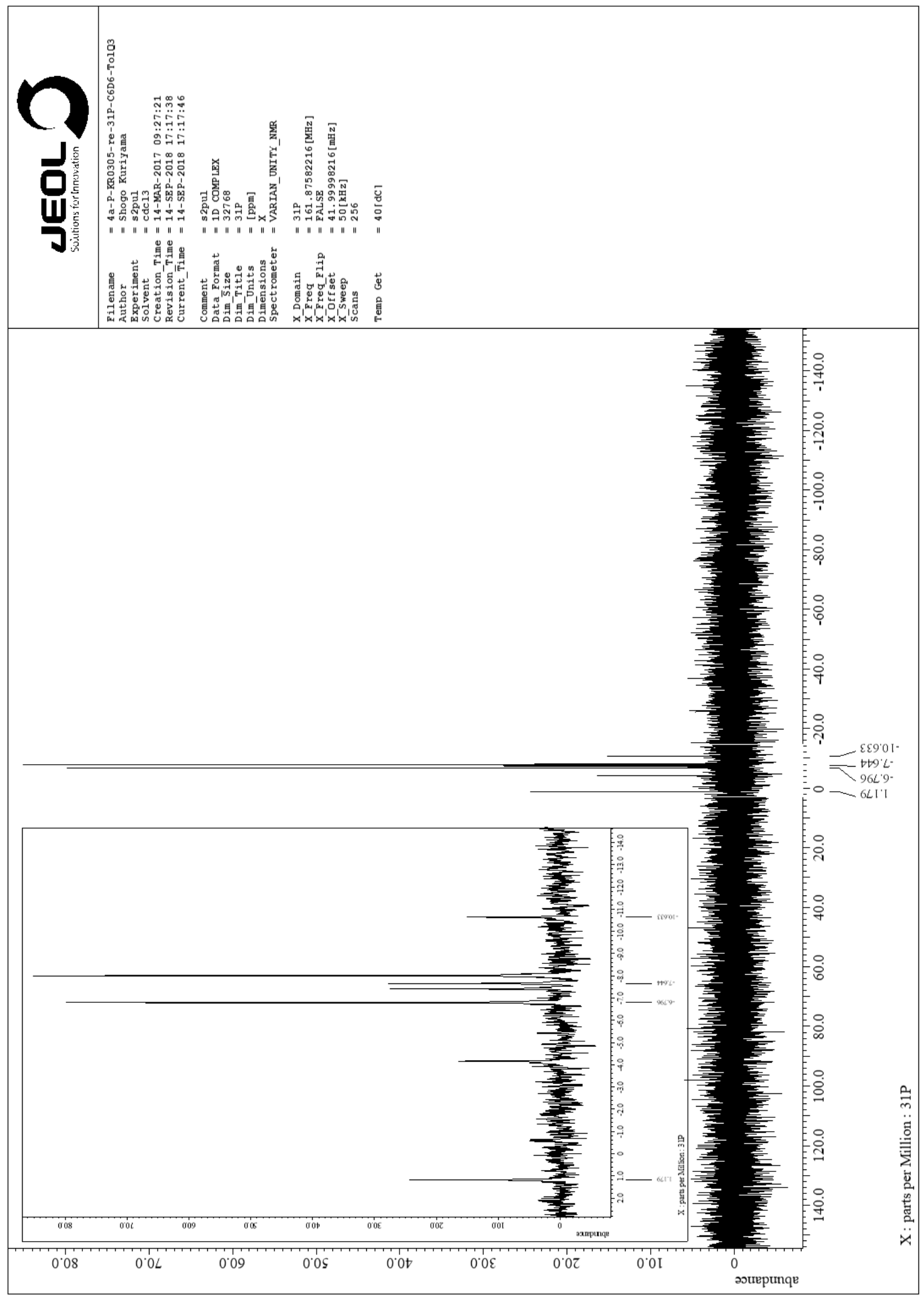

Figure S72. ${ }^{31} \mathrm{P} N M R$ spectrum of $\mathbf{1 a} \cdot 0.3 \mathrm{C}_{4} \mathrm{H}_{8} \mathrm{O} \cdot 0.2 \mathrm{C}_{4} \mathrm{H}_{10} \mathrm{O}$ in $\mathrm{C}_{6} \mathrm{D}_{6}$. 


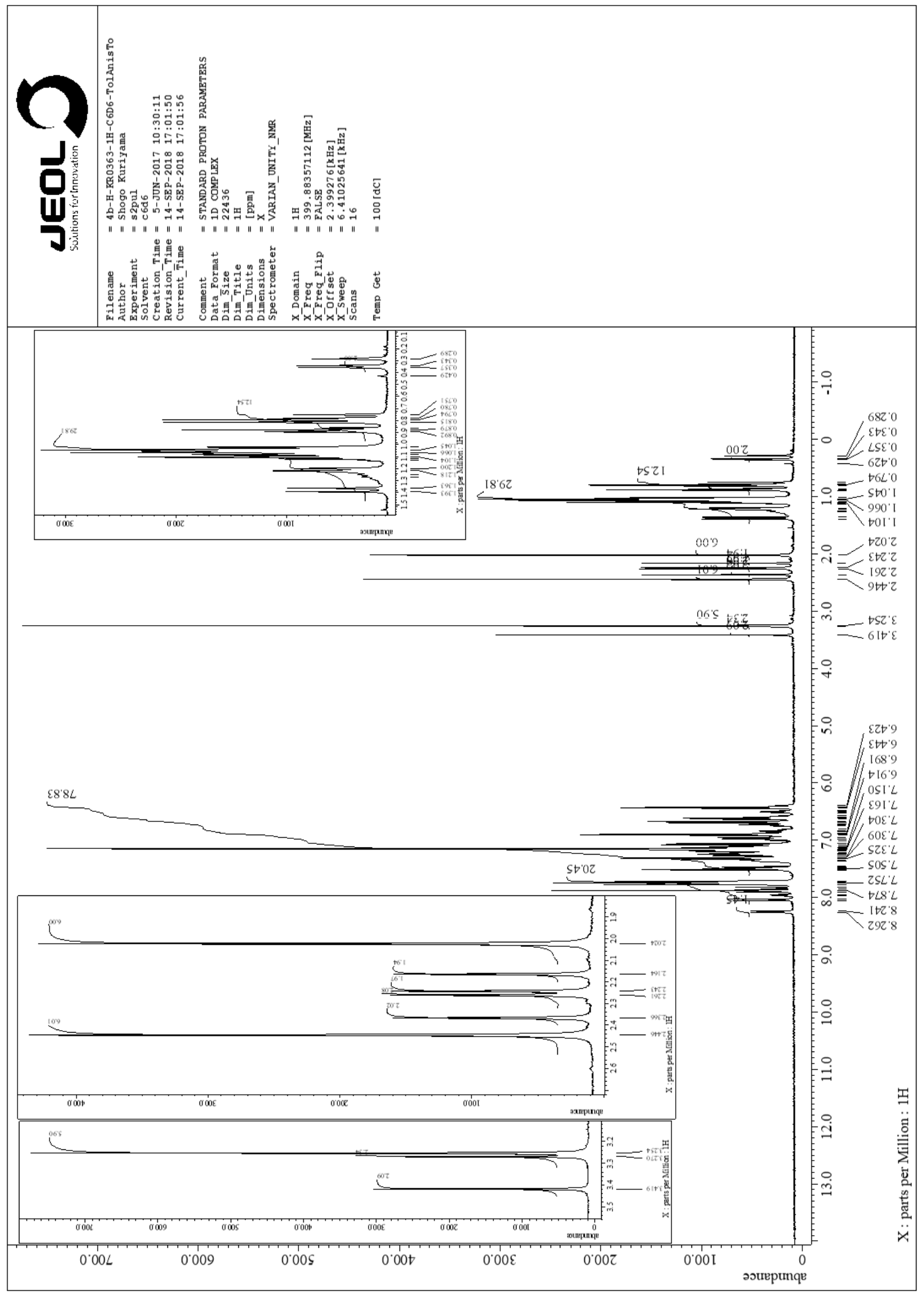

Figure S73. ${ }^{1} \mathrm{H}$ NMR spectrum of $\mathbf{1 b}$ in $\mathrm{C}_{6} \mathrm{D}_{6}$. 


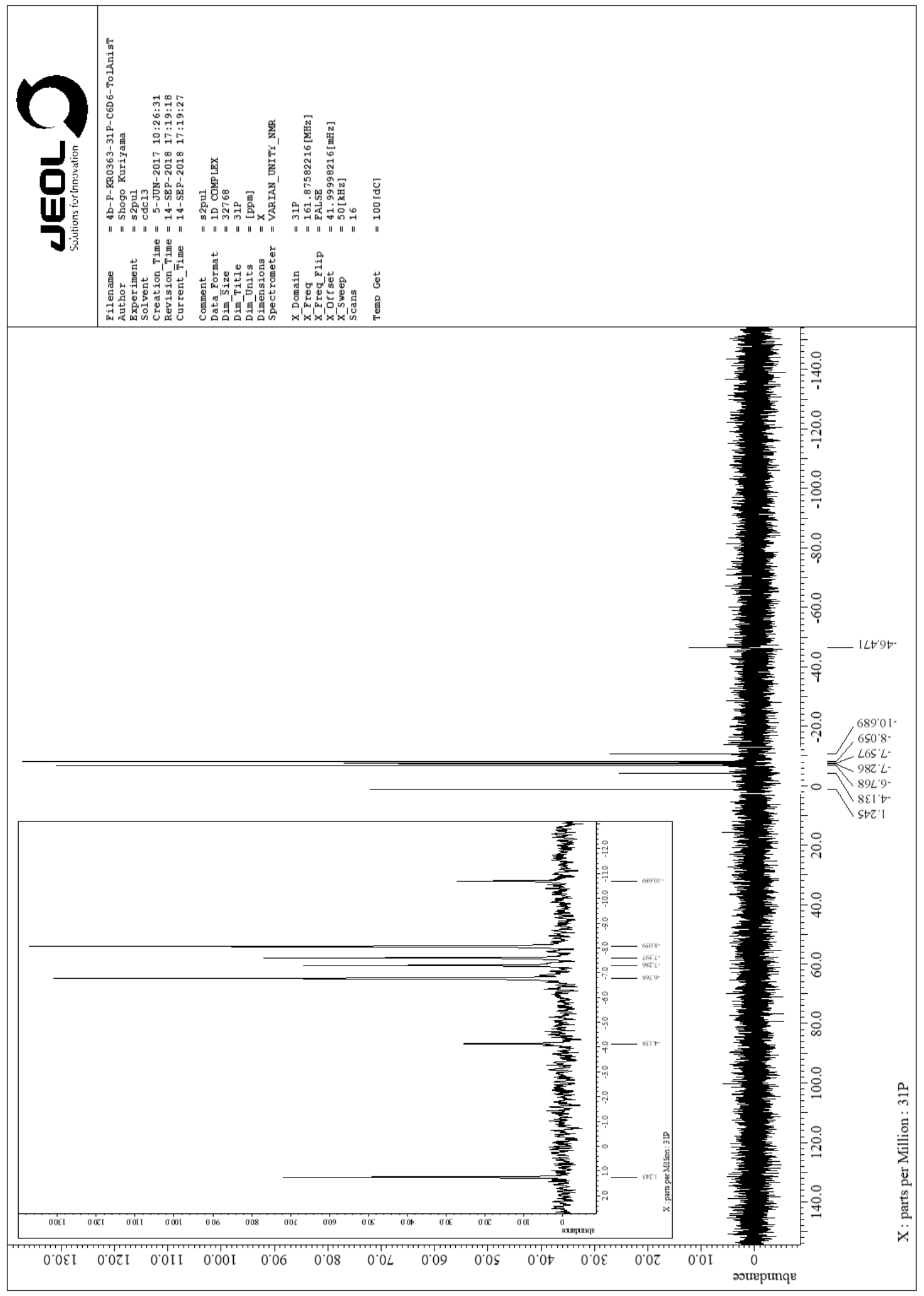

Figure S74. ${ }^{31} \mathrm{P}$ NMR spectrum of $\mathbf{1 b}$ in $\mathrm{C}_{6} \mathrm{D}_{6}$. 


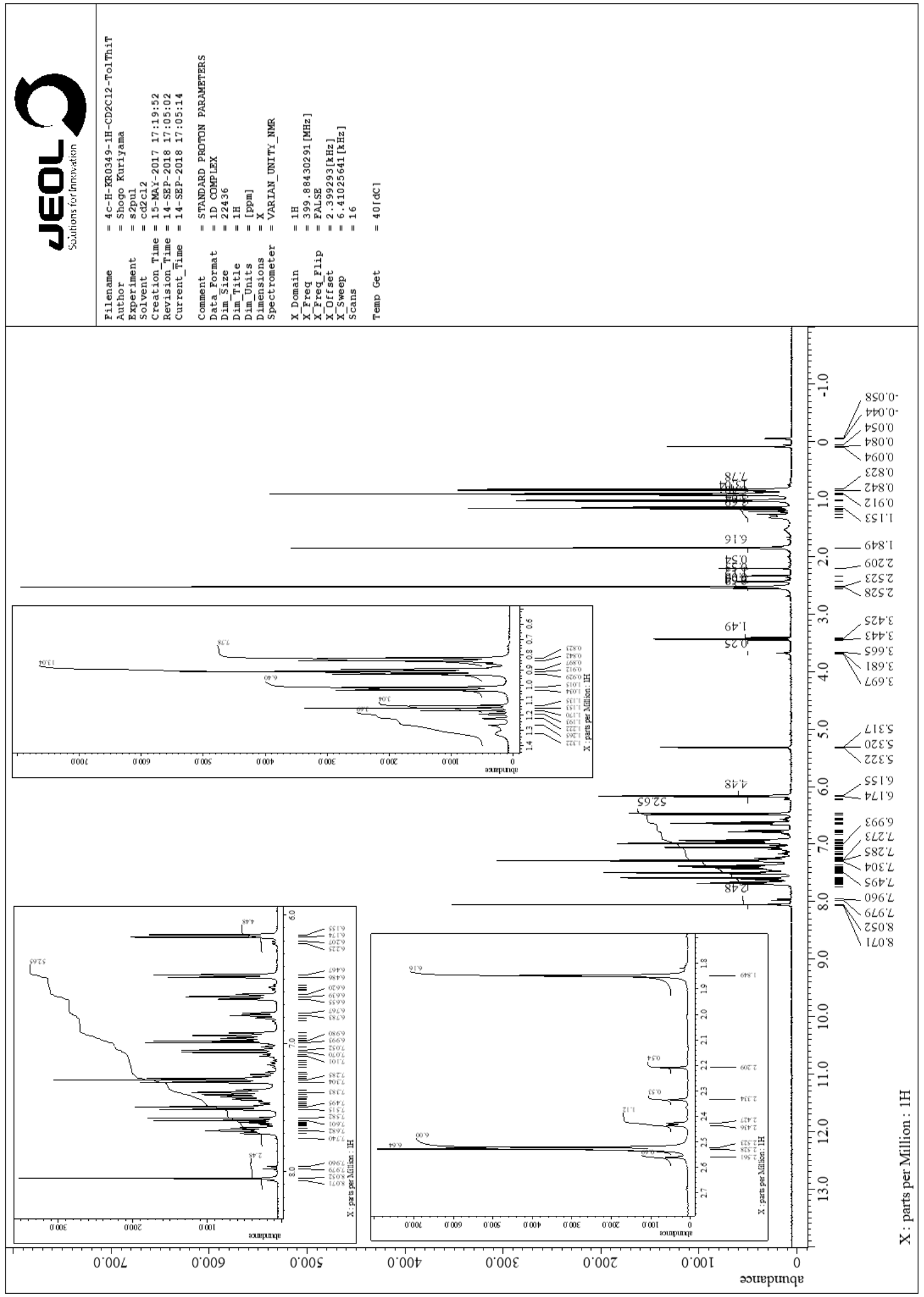

Figure S75. ${ }^{1} \mathrm{H}$ NMR spectrum of $1 \mathbf{c} \cdot 0.33 \mathrm{C}_{4} \mathrm{H}_{10} \mathrm{O}$ in $\mathrm{CD}_{2} \mathrm{Cl}_{2}$. 


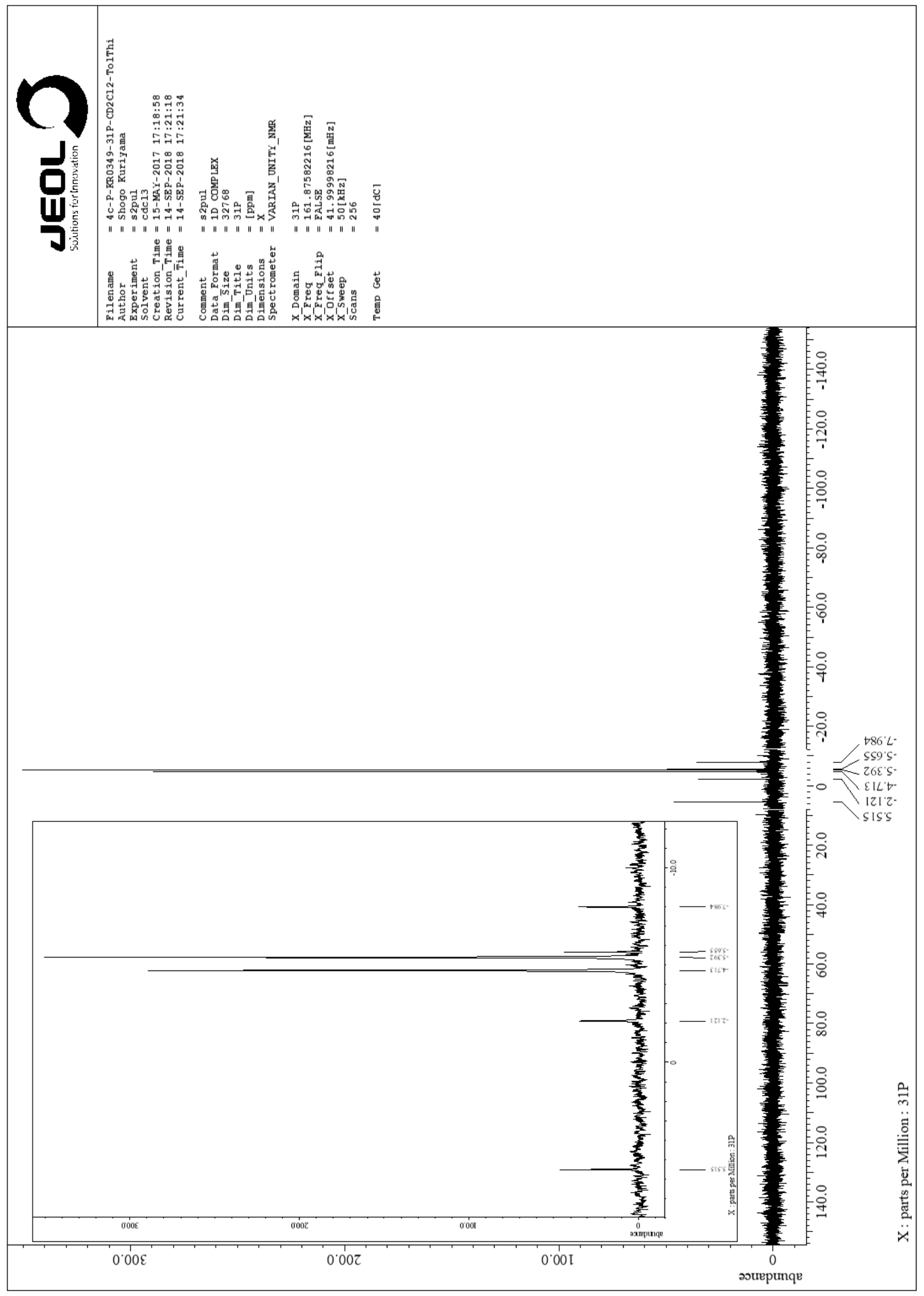

Figure S76. ${ }^{31} \mathrm{P}$ NMR spectrum of $1 \mathrm{c} \cdot 0.33 \mathrm{C}_{4} \mathrm{H}_{10} \mathrm{O}$ in $\mathrm{CD}_{2} \mathrm{Cl}_{2}$. 


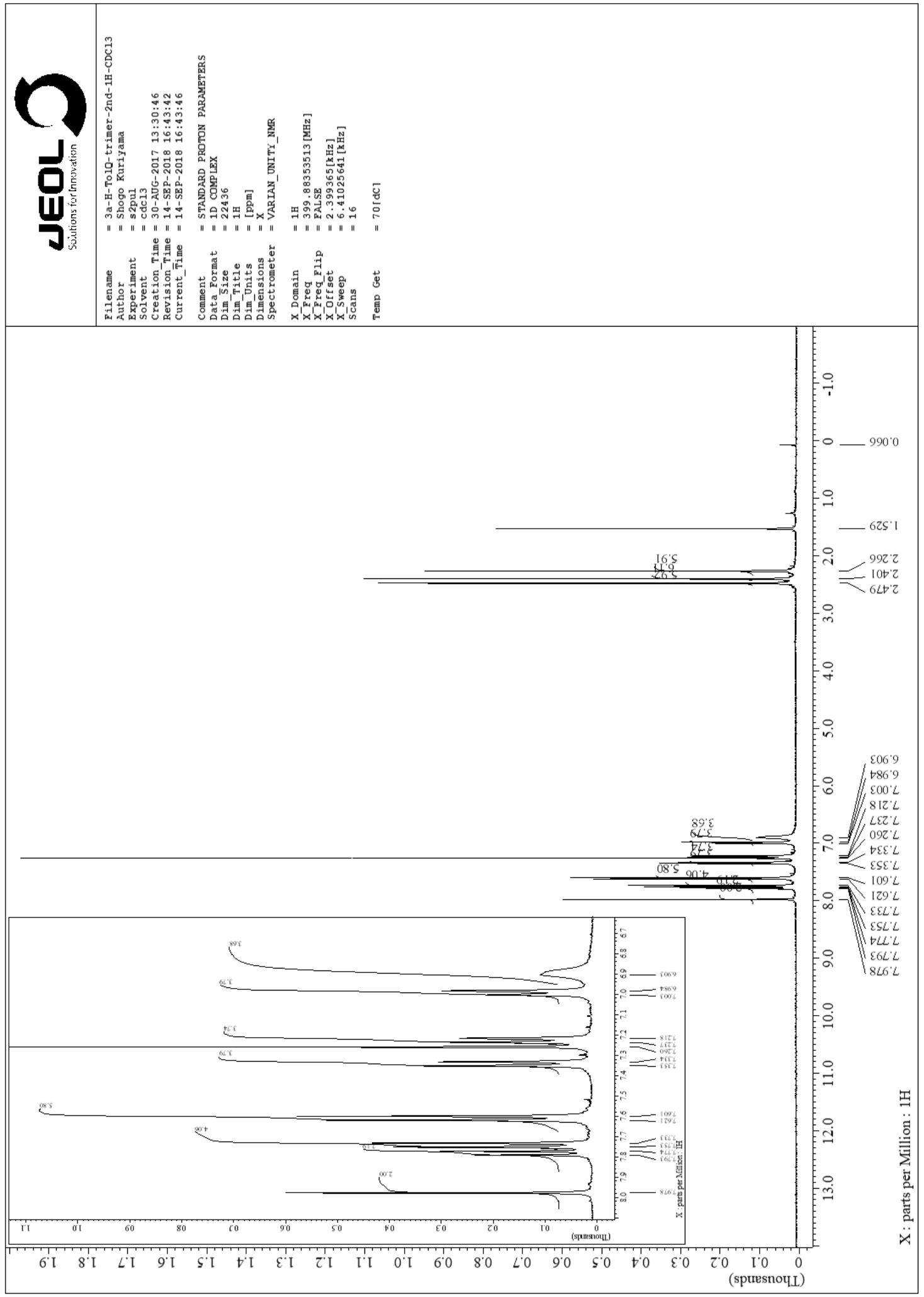

Figure S77. ${ }^{1} \mathrm{H}$ NMR spectrum of $\mathbf{2 a}$ in $\mathrm{CDCl}_{3}$. 


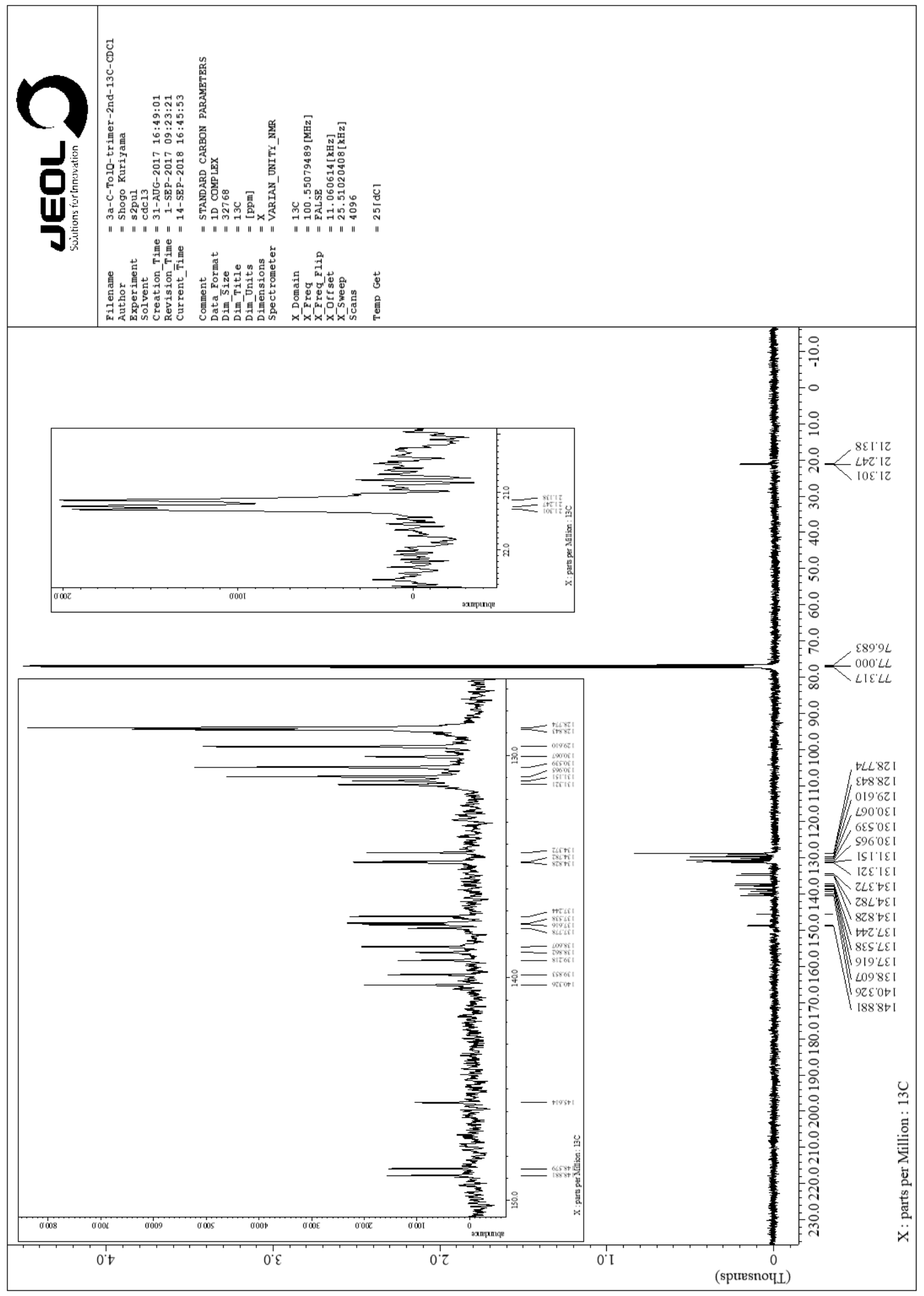

Figure S78. ${ }^{13} \mathrm{C}$ NMR spectrum of $\mathbf{2 a}$ in $\mathrm{CDCl}_{3}$. 


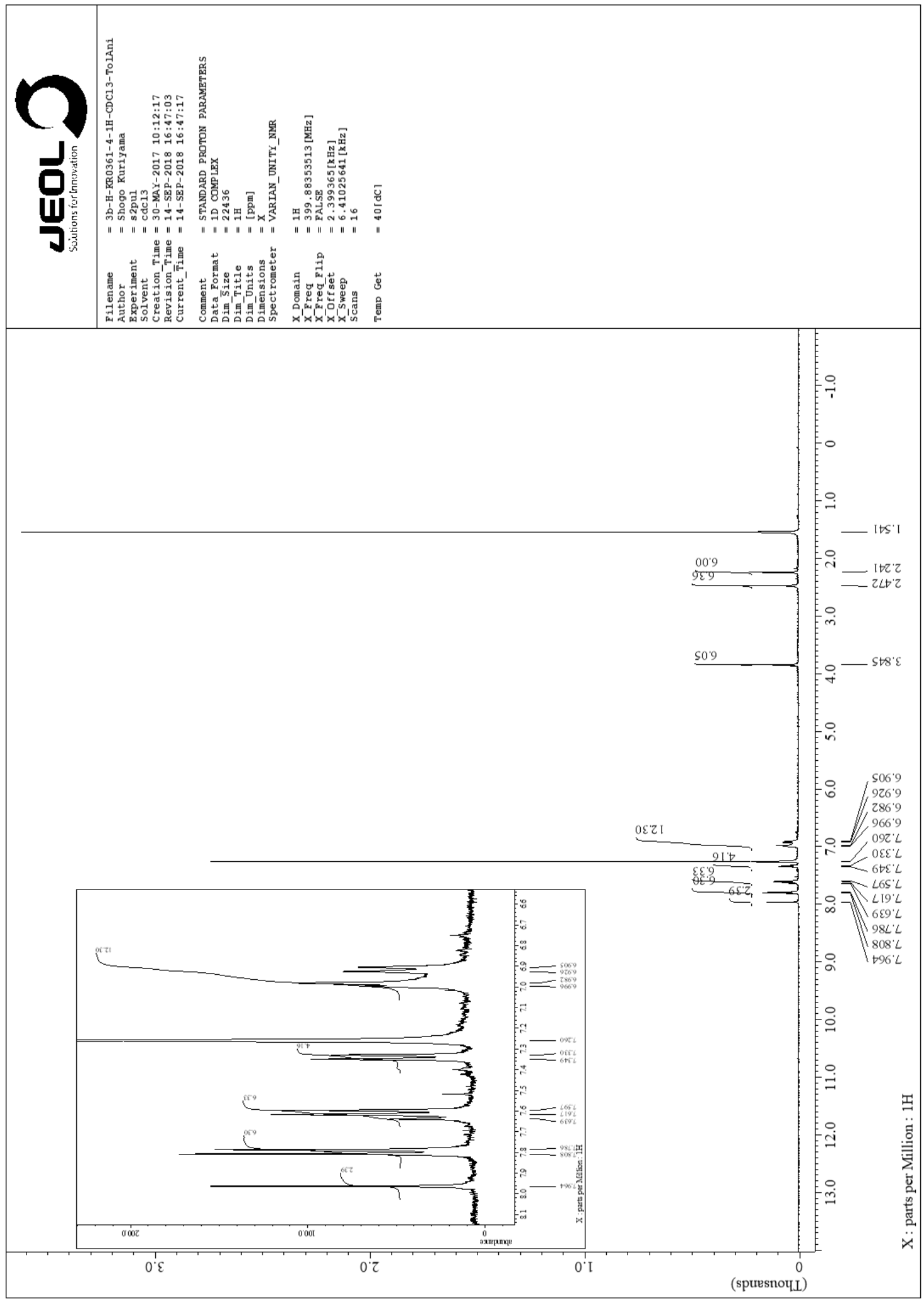

Figure S79. ${ }^{1} \mathrm{H}$ NMR spectra of $\mathbf{2 b}$ in $\mathrm{CDCl}_{3}$. 


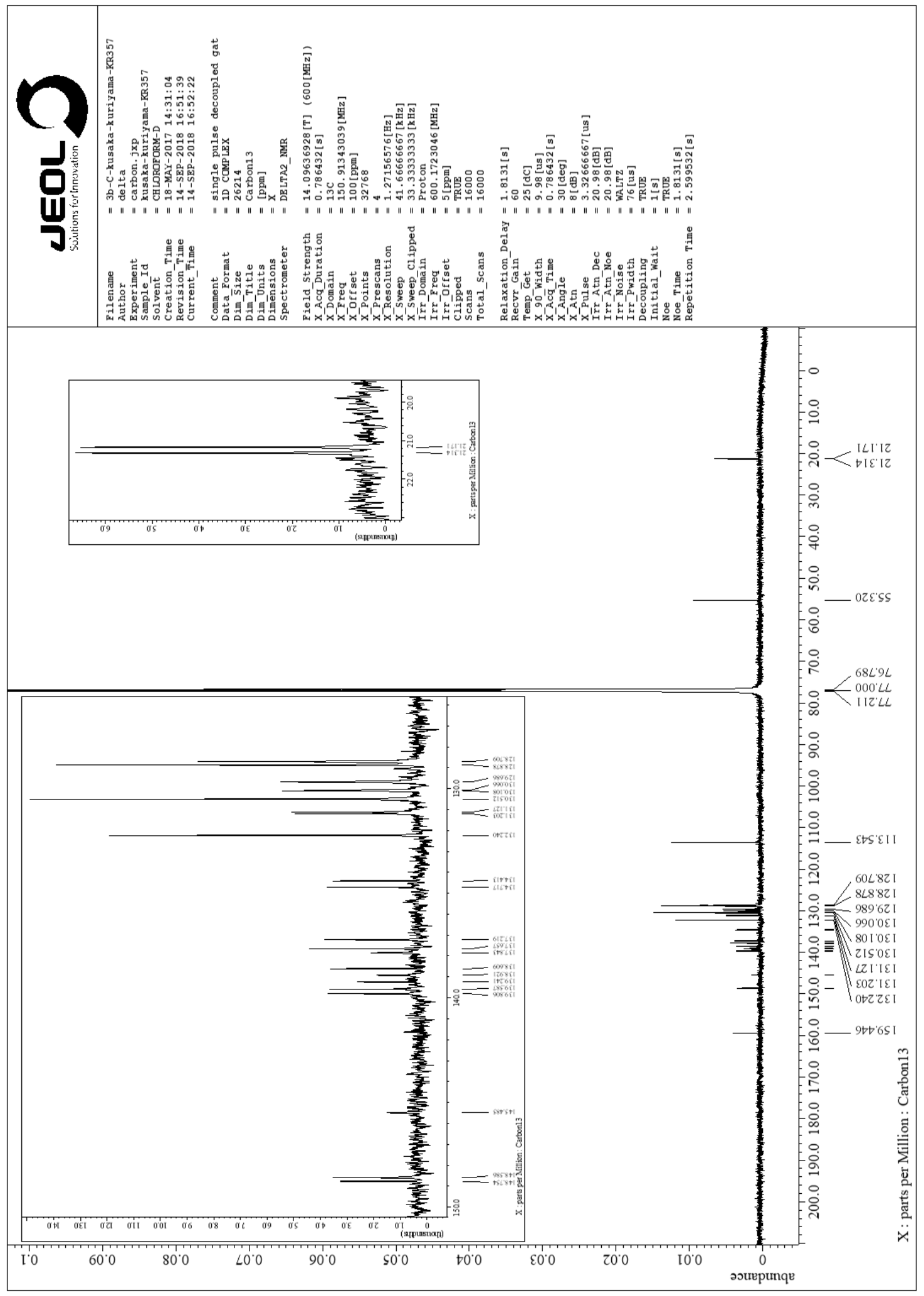

Figure S80. ${ }^{13} \mathrm{C}$ NMR spectra of $\mathbf{2} \mathbf{b}$ in $\mathrm{CDCl}_{3}$. 


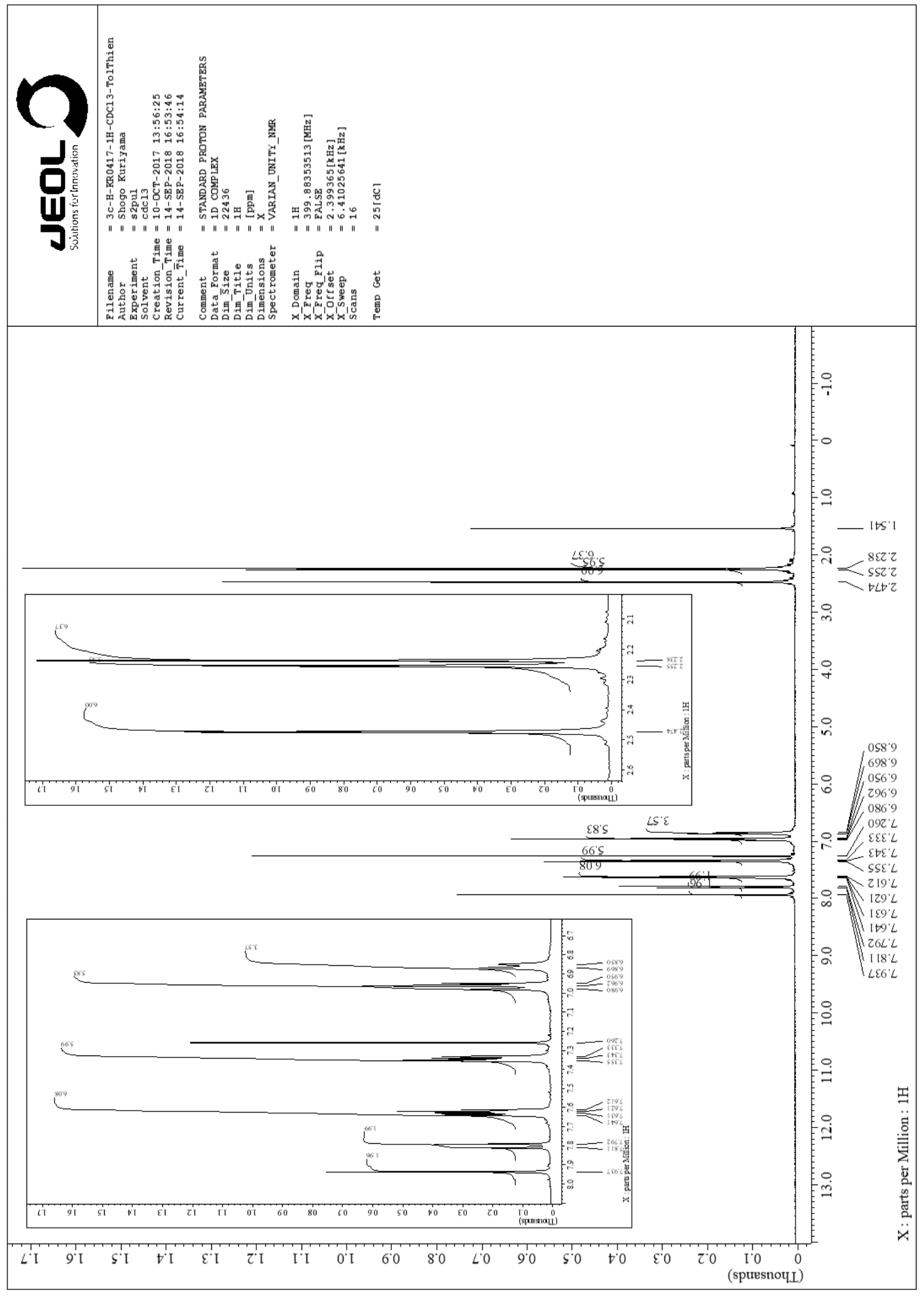

Figure S81. ${ }^{1} \mathrm{H}$ NMR spectrum of $2 \mathbf{c}$ in $\mathrm{CDCl}_{3}$. 


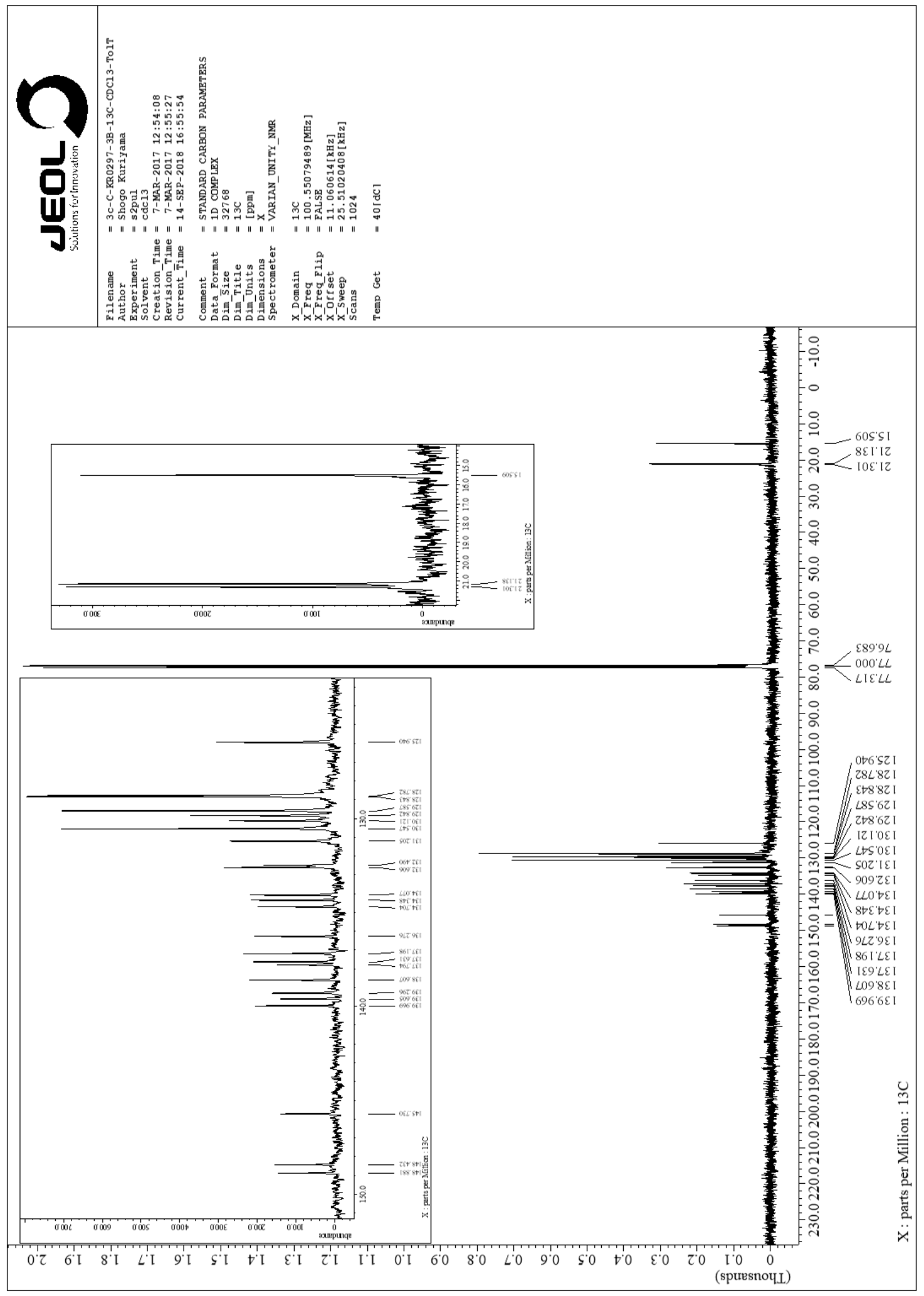

Figure S82. ${ }^{13} \mathrm{C}$ NMR spectrum of $\mathbf{2 c}$ in $\mathrm{CDCl}_{3}$. 


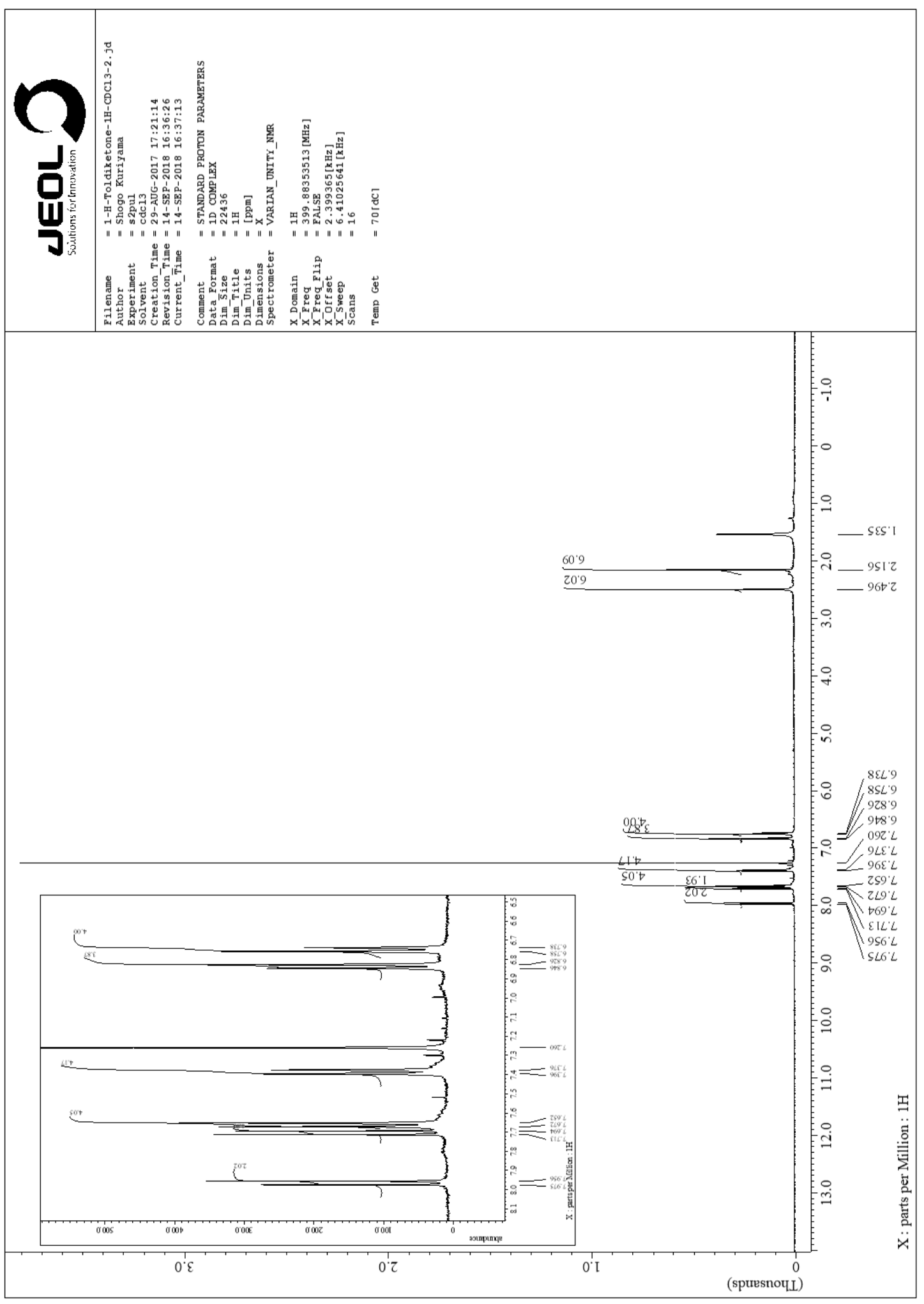

Figure S83. ${ }^{1} \mathrm{H}$ NMR spectrum of $\mathbf{3}$ in $\mathrm{CDCl}_{3}$. 


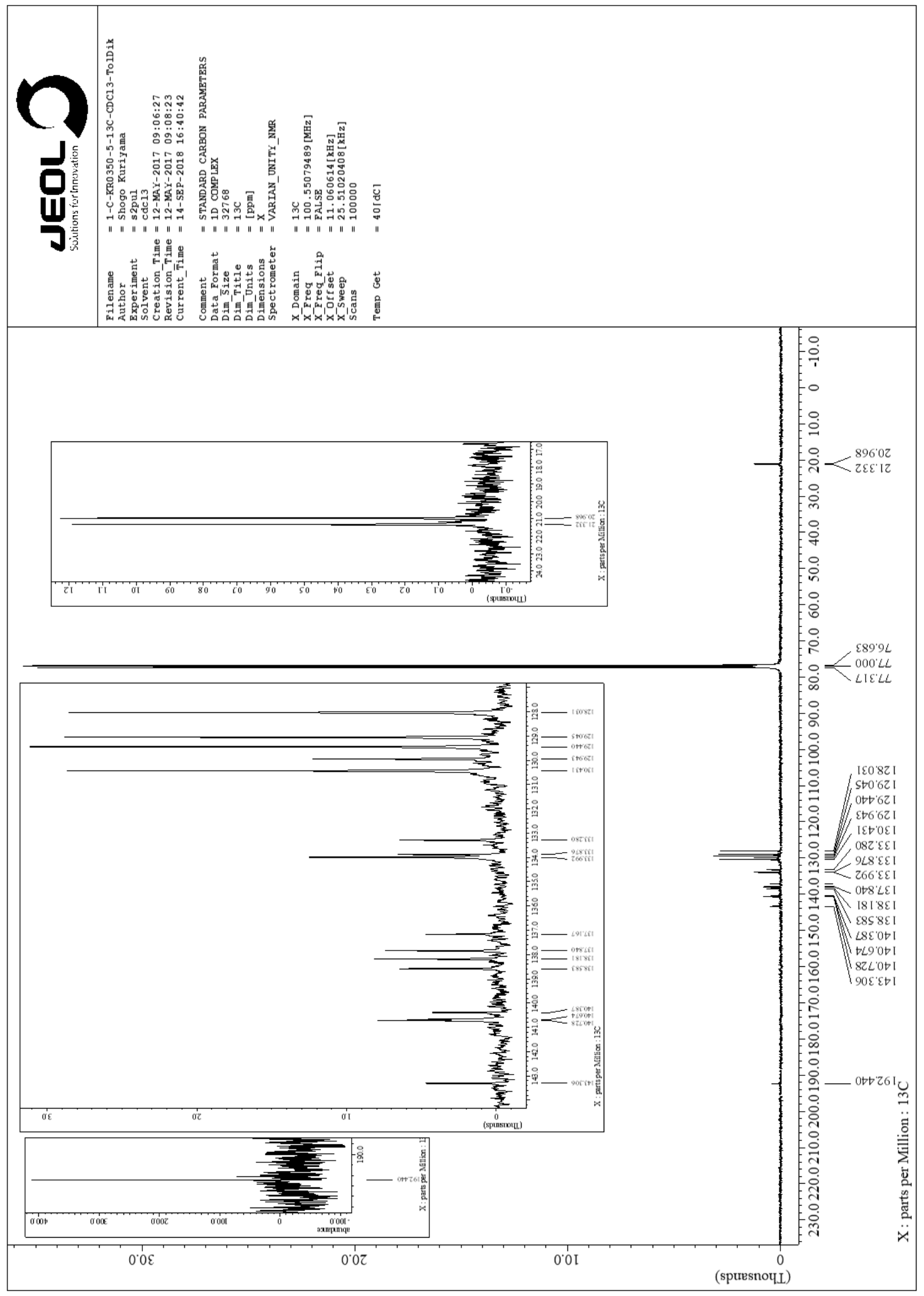

Figure S84. ${ }^{13} \mathrm{C}$ NMR spectrum of 3 in $\mathrm{CDCl}_{3}$. 


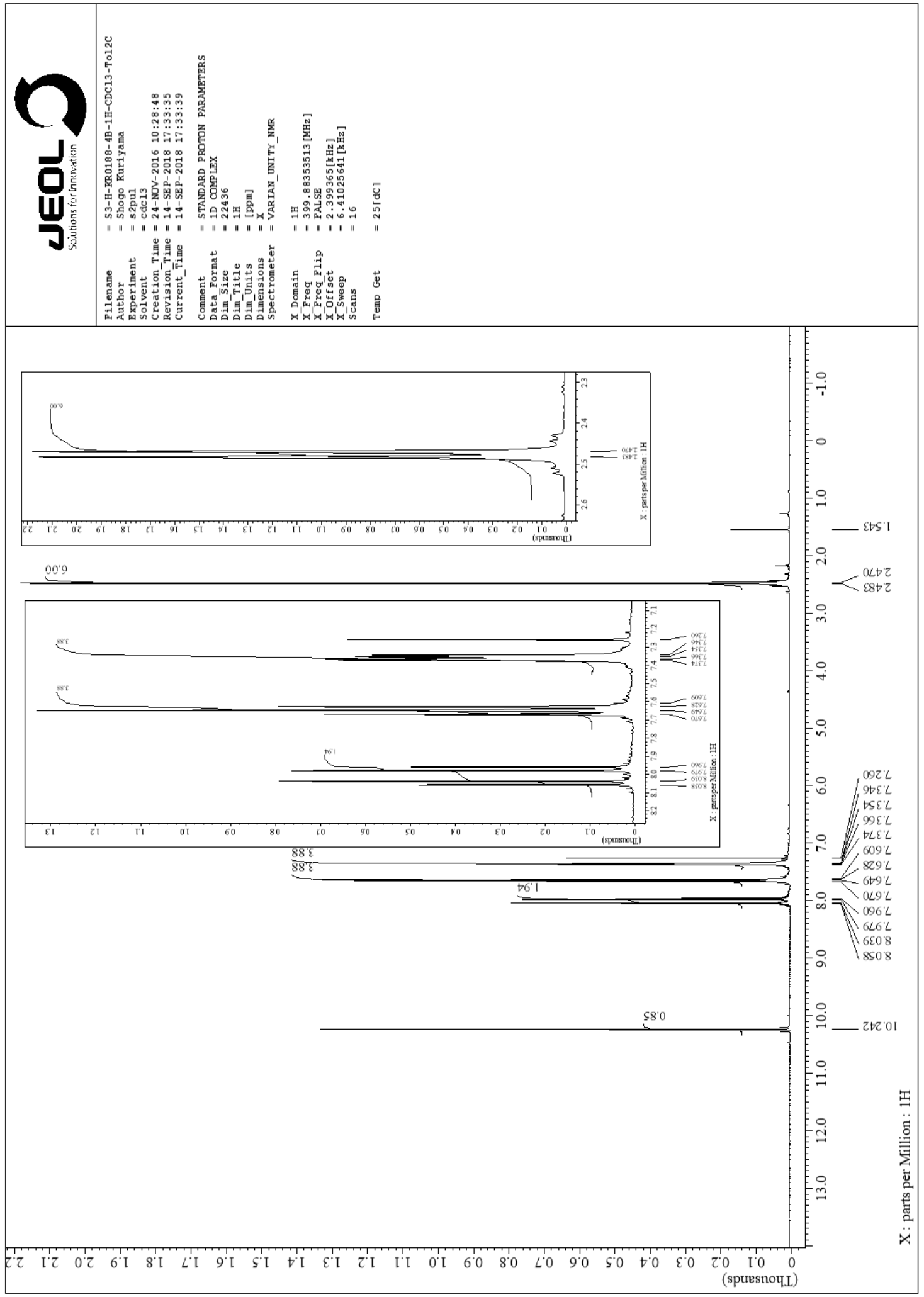

Figure S85. ${ }^{1} \mathrm{H}$ NMR spectrum of 5 in $\mathrm{CDCl}_{3}$. 


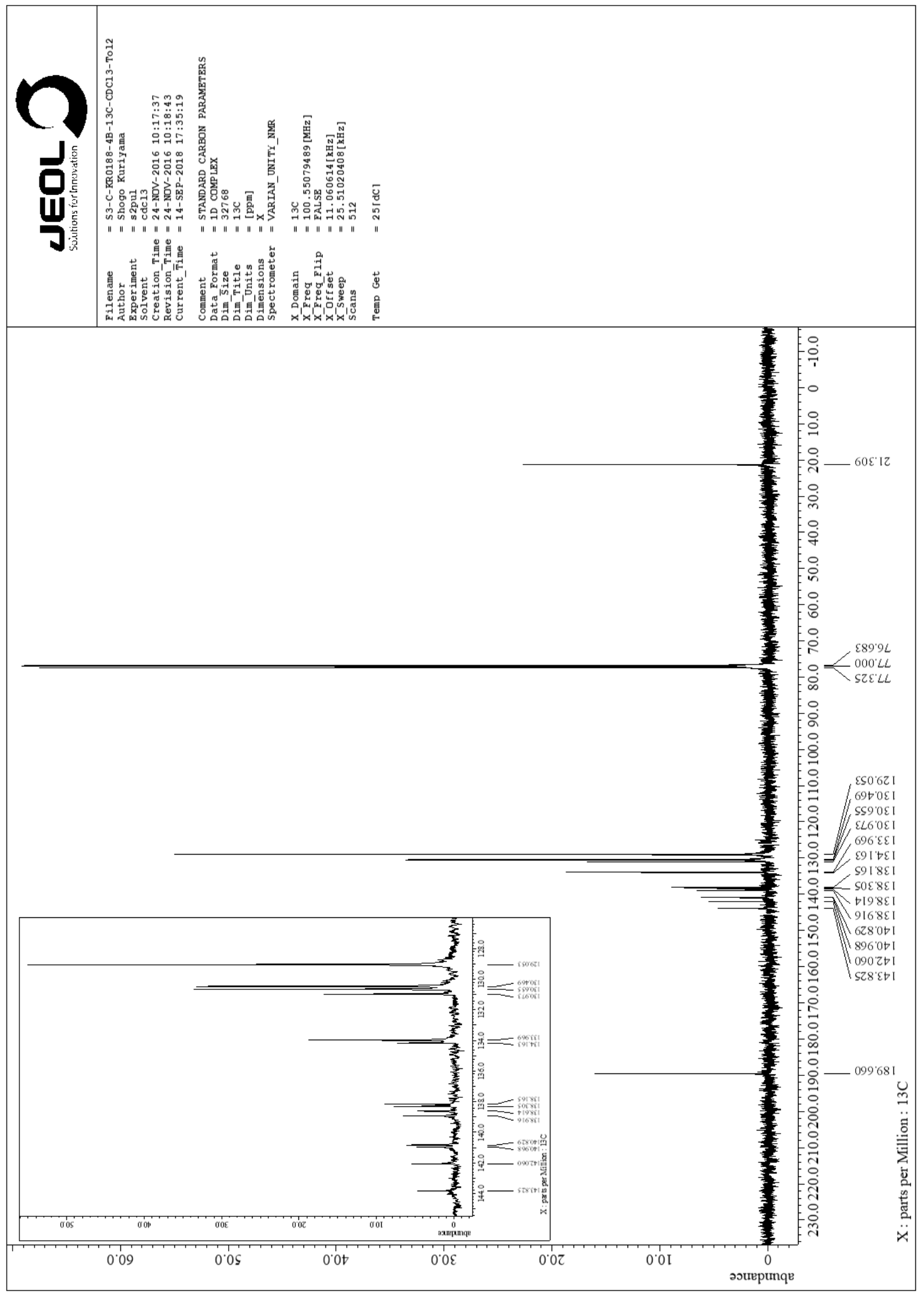

Figure S86. ${ }^{13} \mathrm{C}$ NMR spectrum of 5 in $\mathrm{CDCl}_{3}$. 


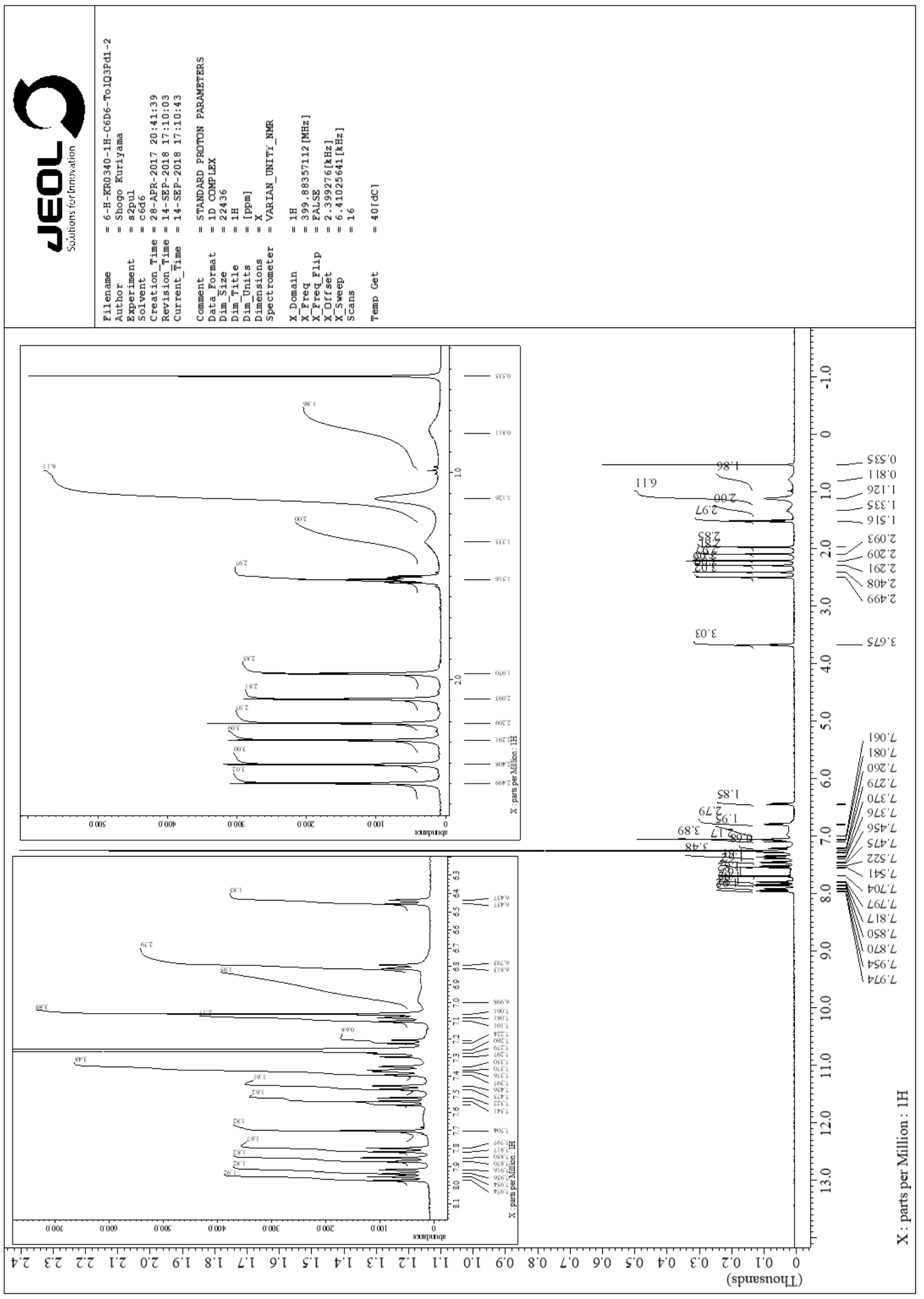

Figure S87. ${ }^{1} \mathrm{H}$ NMR spectrum of $\mathbf{6 a} \cdot 0.75 \mathrm{C}_{4} \mathrm{H}_{8} \mathrm{O}$ in $\mathrm{C}_{6} \mathrm{D}_{6}$. 


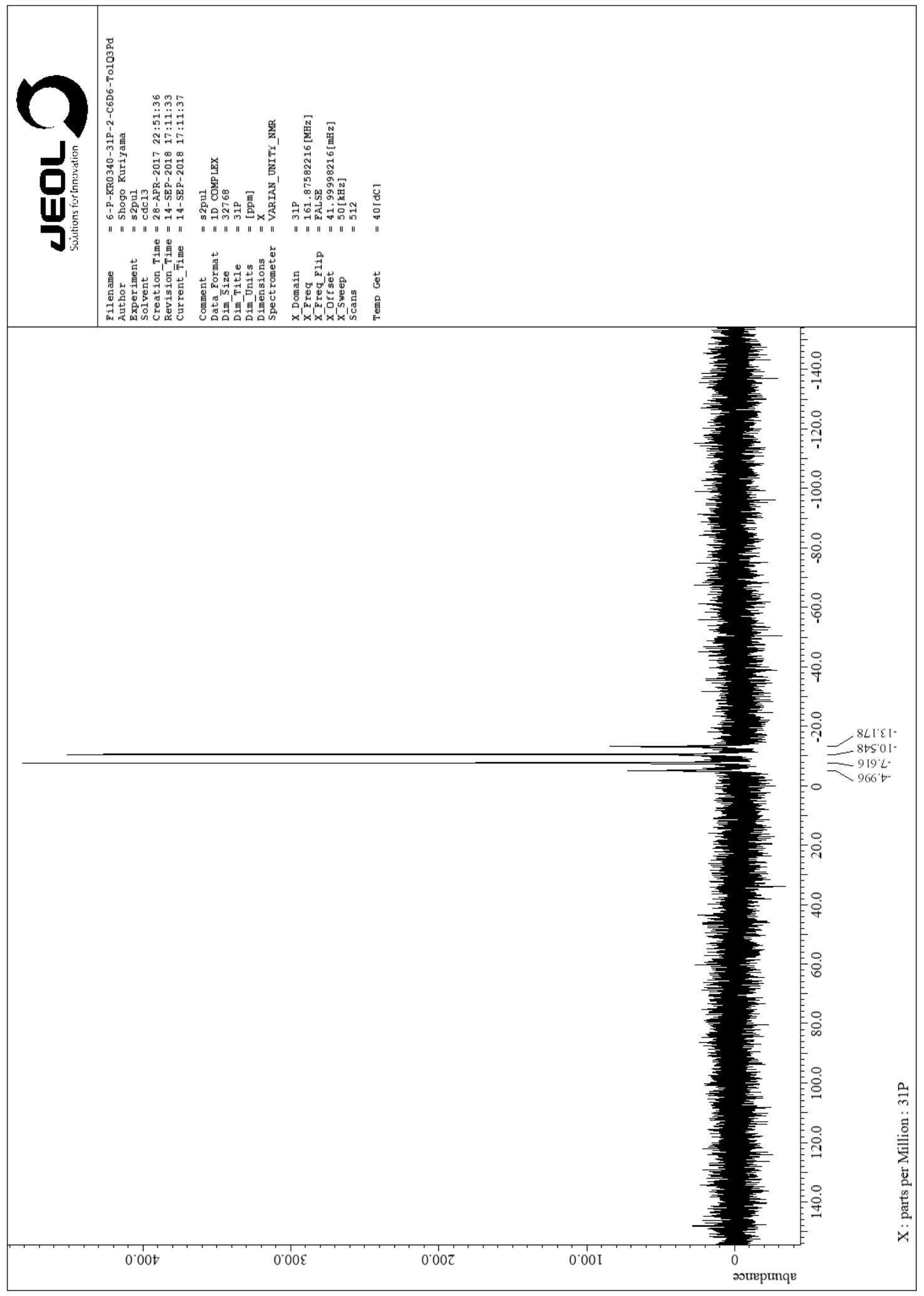

Figure S88. ${ }^{31} \mathrm{P}$ NMR spectrum of $\mathbf{6 a} \cdot 0.75 \mathrm{C}_{4} \mathrm{H}_{8} \mathrm{O}$ in $\mathrm{C}_{6} \mathrm{D}_{6}$. 


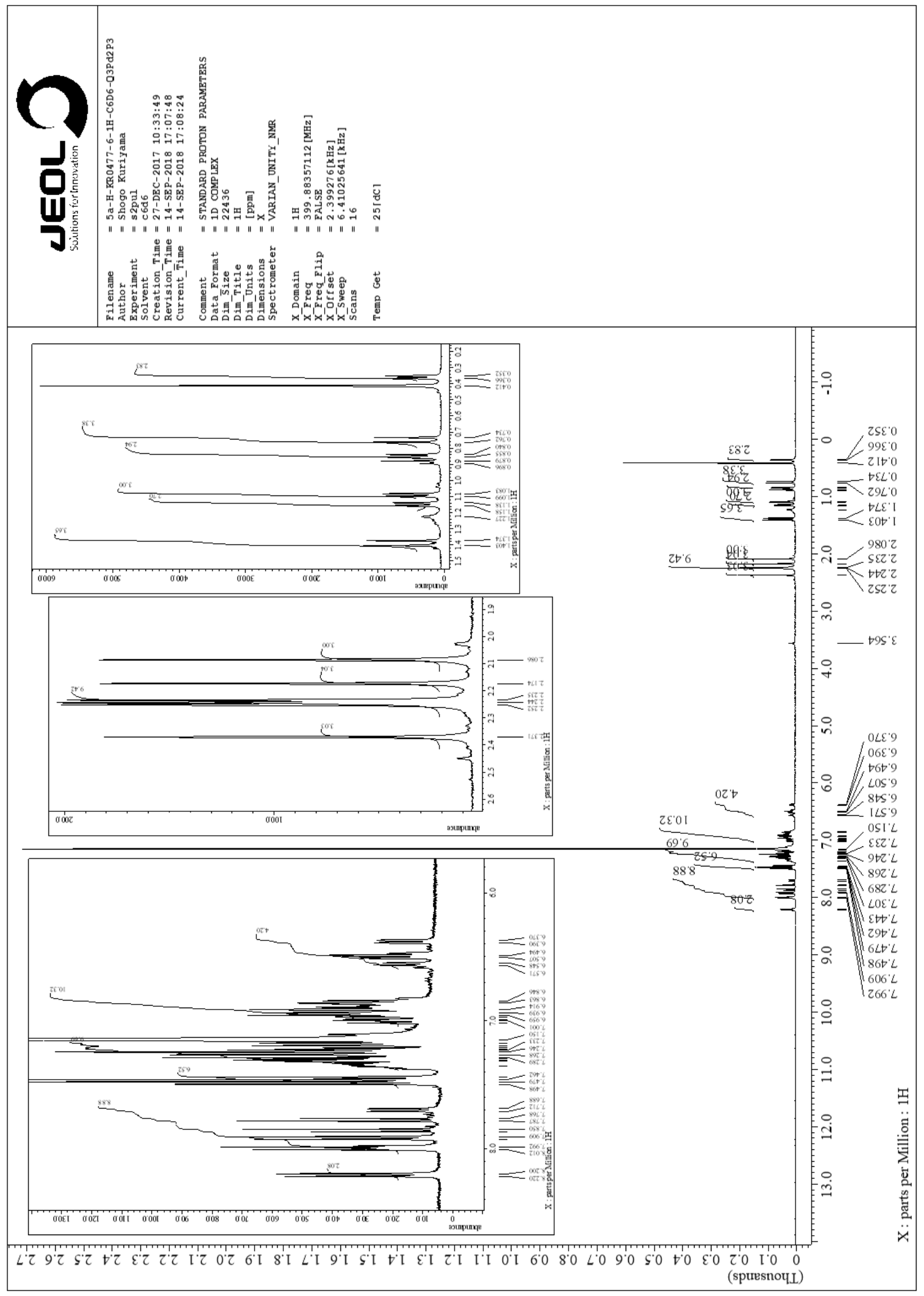

Figure S89. ${ }^{1} \mathrm{H}$ NMR spectrum of 7a in $\mathrm{C}_{6} \mathrm{D}_{6}$. 


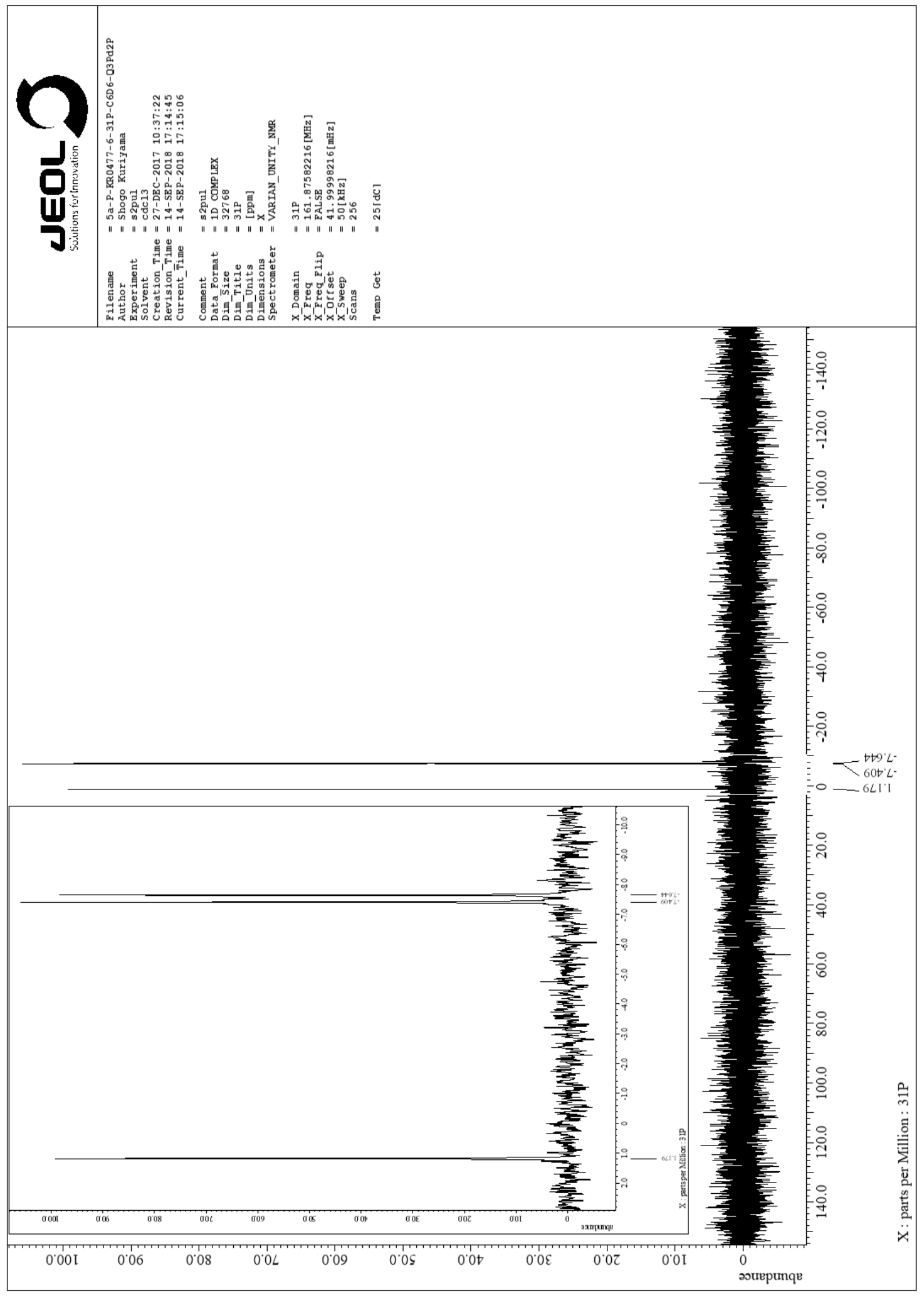

Figure S90. ${ }^{31} \mathrm{P}$ NMR spectrum of $7 \mathbf{a}$ in $\mathrm{C}_{6} \mathrm{D}_{6}$. 


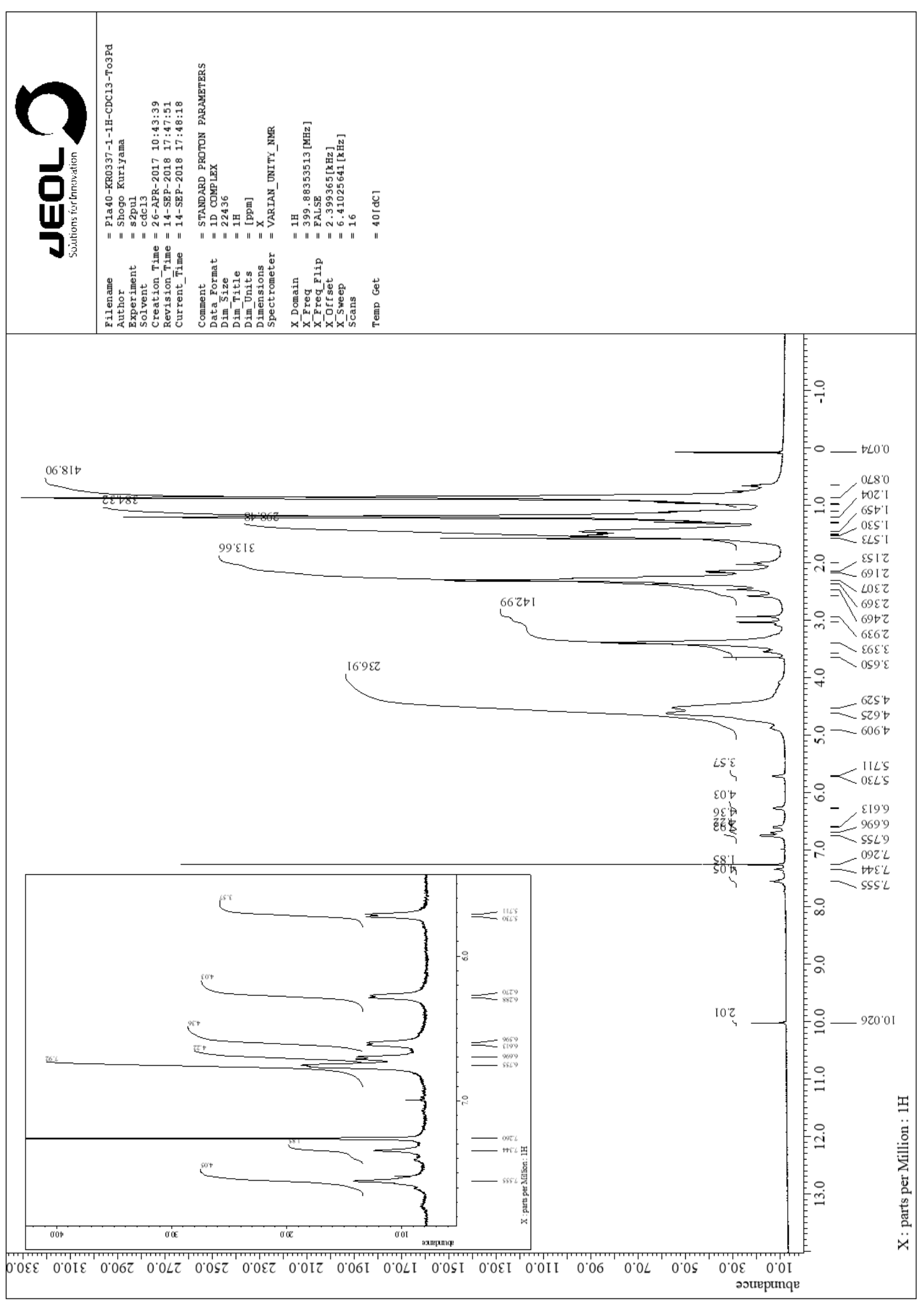

Figure S91. ${ }^{1} \mathrm{H}$ NMR spectrum of $\mathbf{P 1 a ( 4 0 )}$ in $\mathrm{CDCl}_{3}$. 


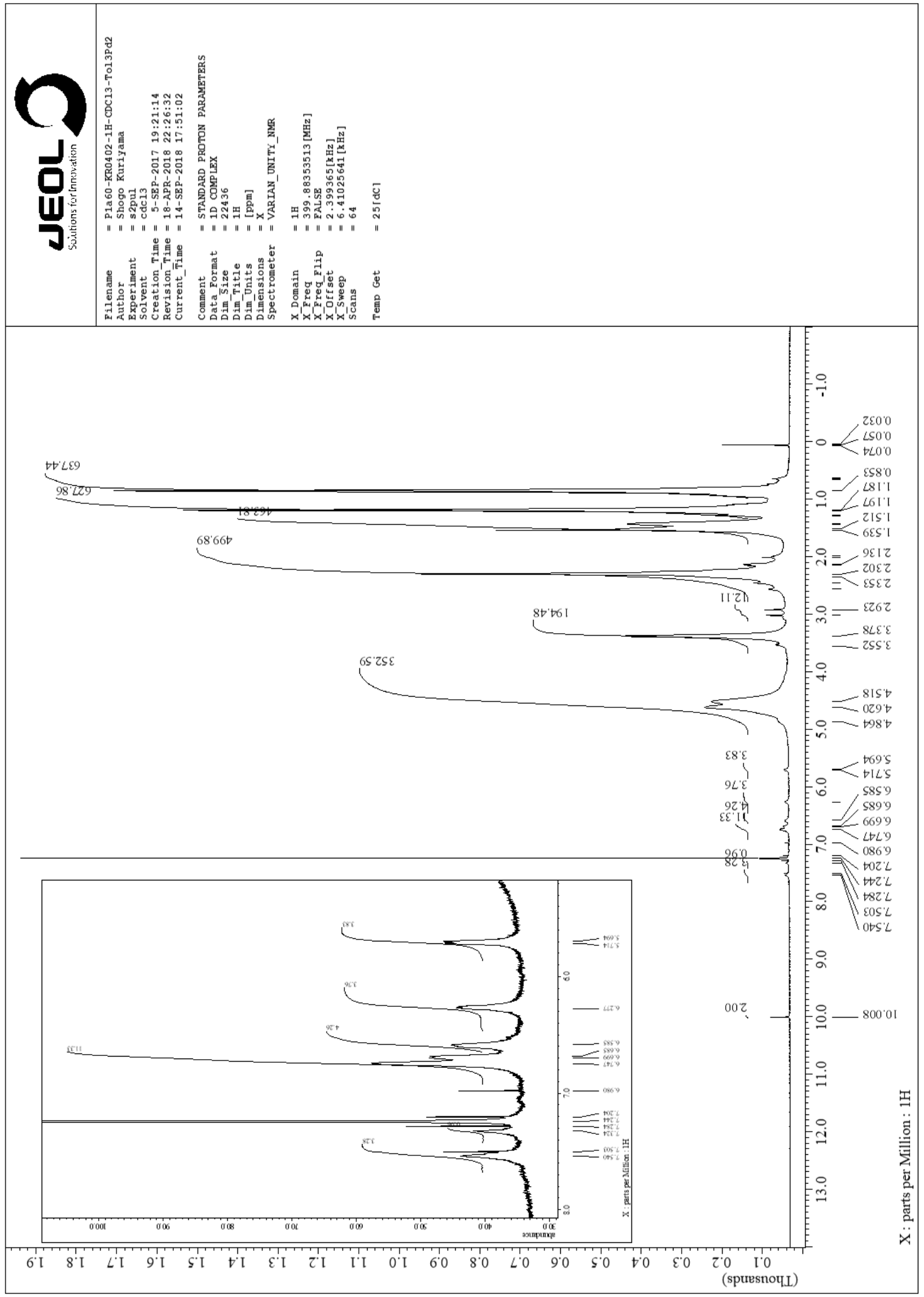

Figure S92. ${ }^{1} \mathrm{H}$ NMR spectrum of $\mathbf{P 1 a ( 6 0 )}$ in $\mathrm{CDCl}_{3}$. 


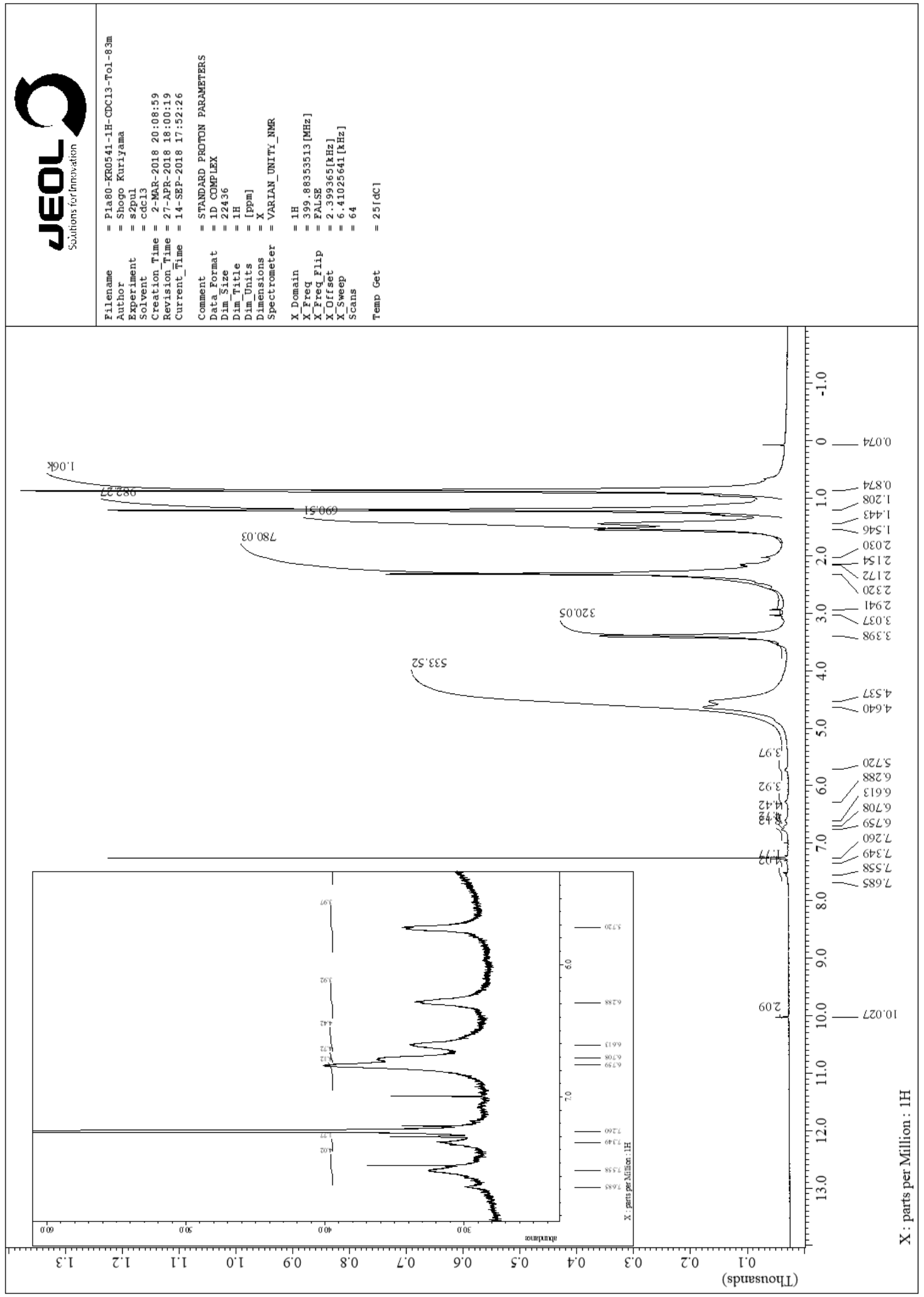

Figure S93. ${ }^{1} \mathrm{H}$ NMR spectrum of $\mathbf{P 1 a}(\mathbf{8 0})$ in $\mathrm{CDCl}_{3}$. 


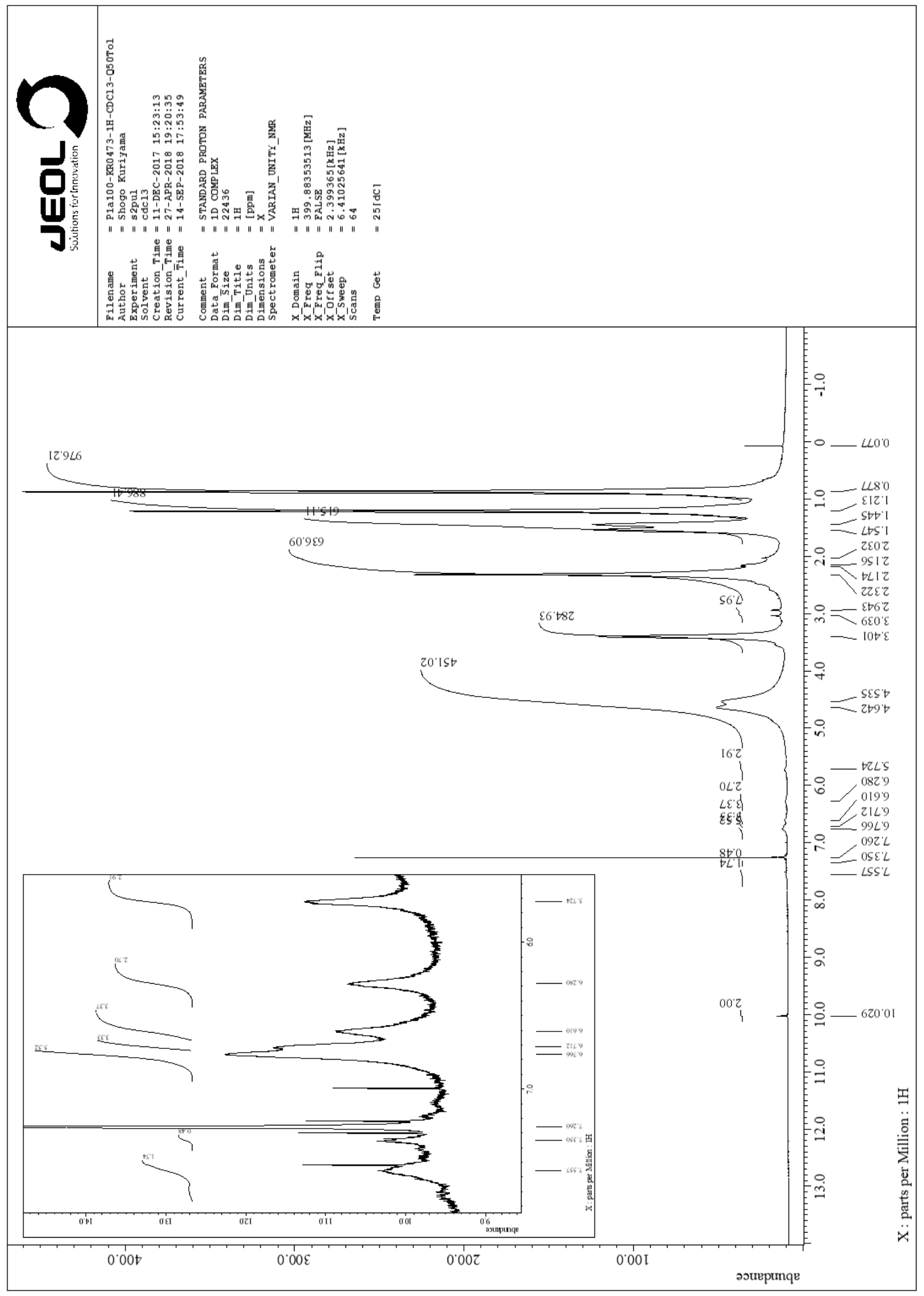

Figure S94. ${ }^{1} \mathrm{H}$ NMR spectrum of P1a(100) in $\mathrm{CDCl}_{3}$. 


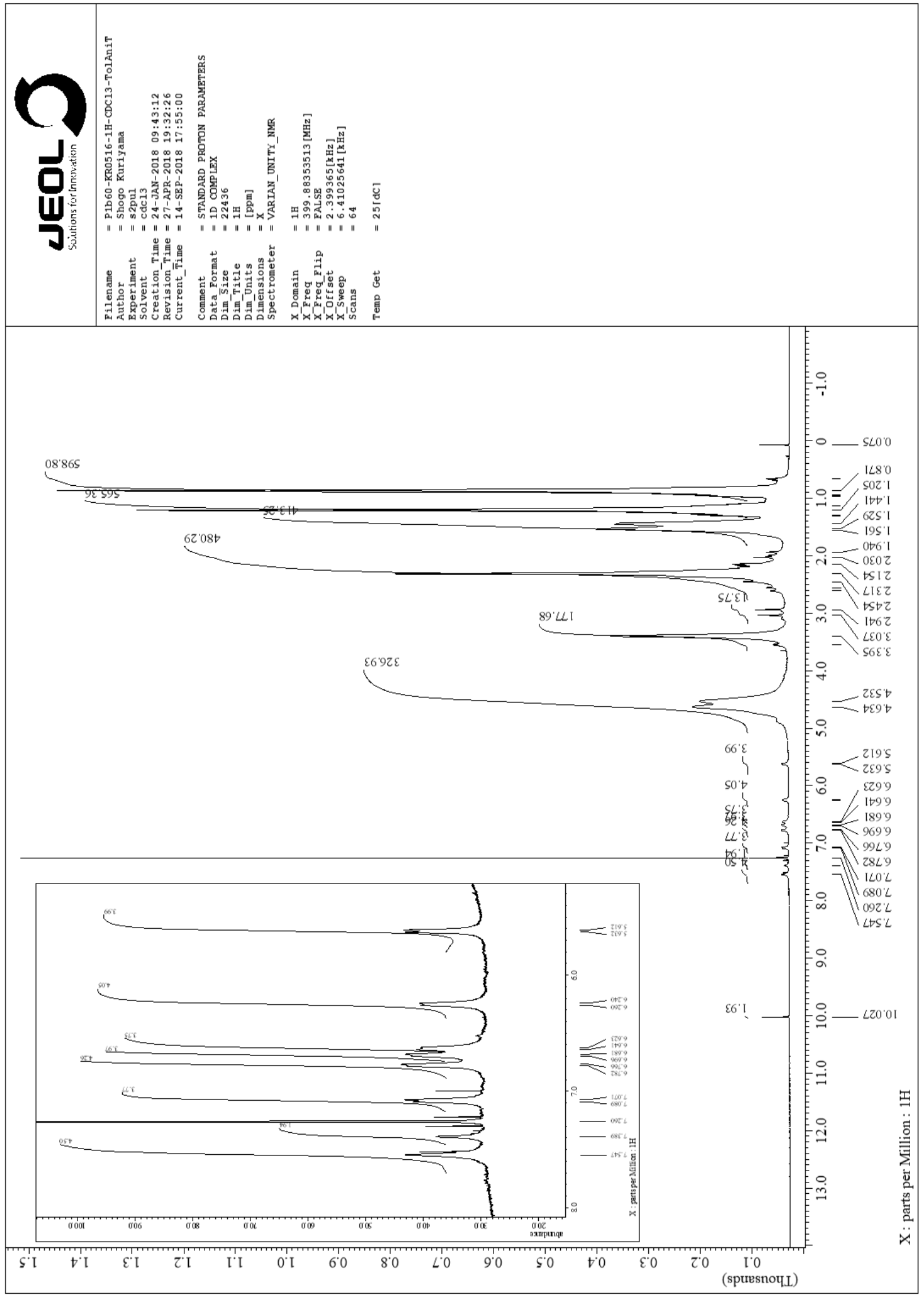

Figure S95. ${ }^{1} \mathrm{H}$ NMR spectrum of $\mathbf{P 1 b}(\mathbf{6 0})$ in $\mathrm{CDCl}_{3}$. 


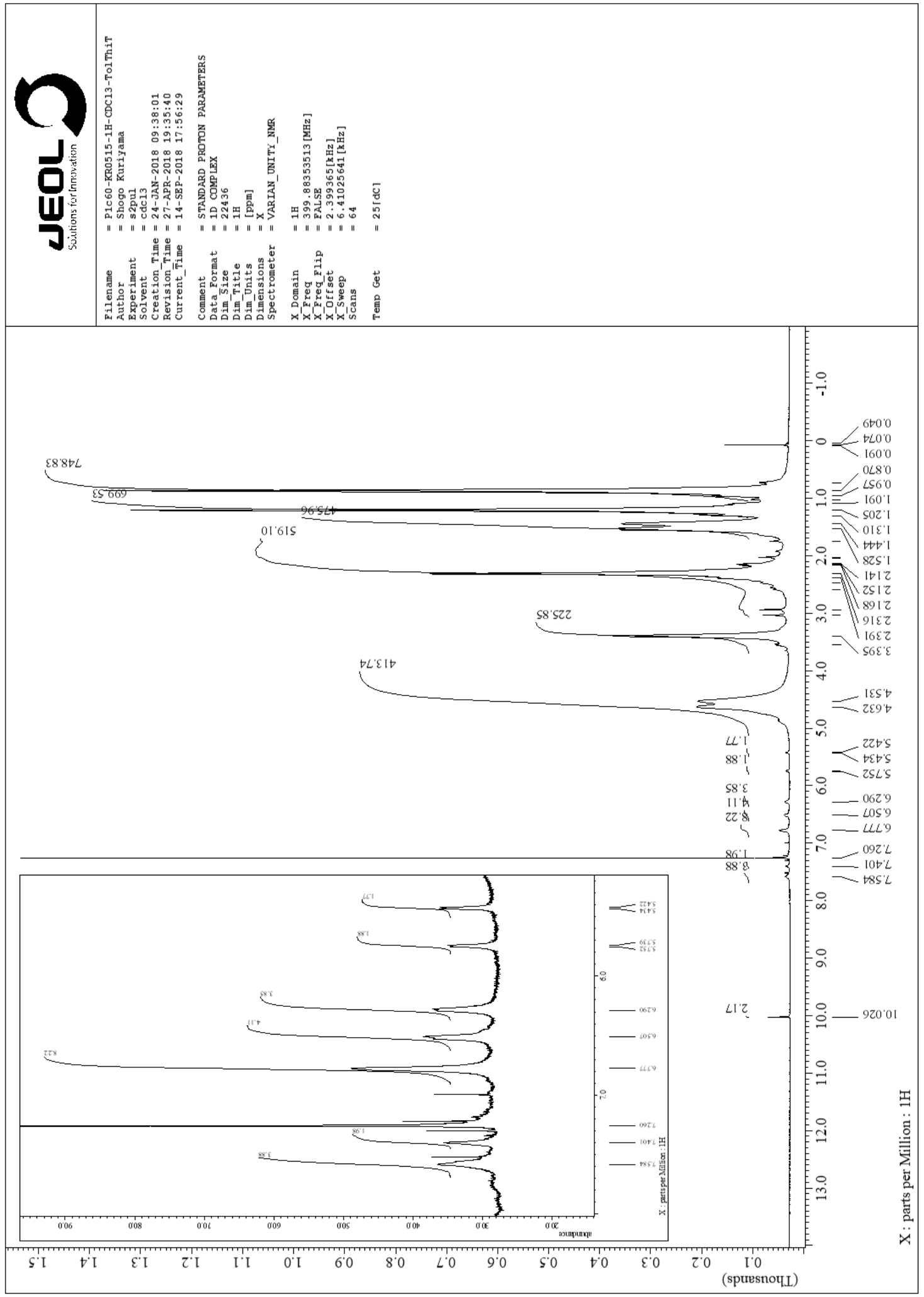

Figure S96. ${ }^{1} \mathrm{H}$ NMR spectrum of $\mathbf{P 1 c}(60)$ in $\mathrm{CDCl}_{3}$. 


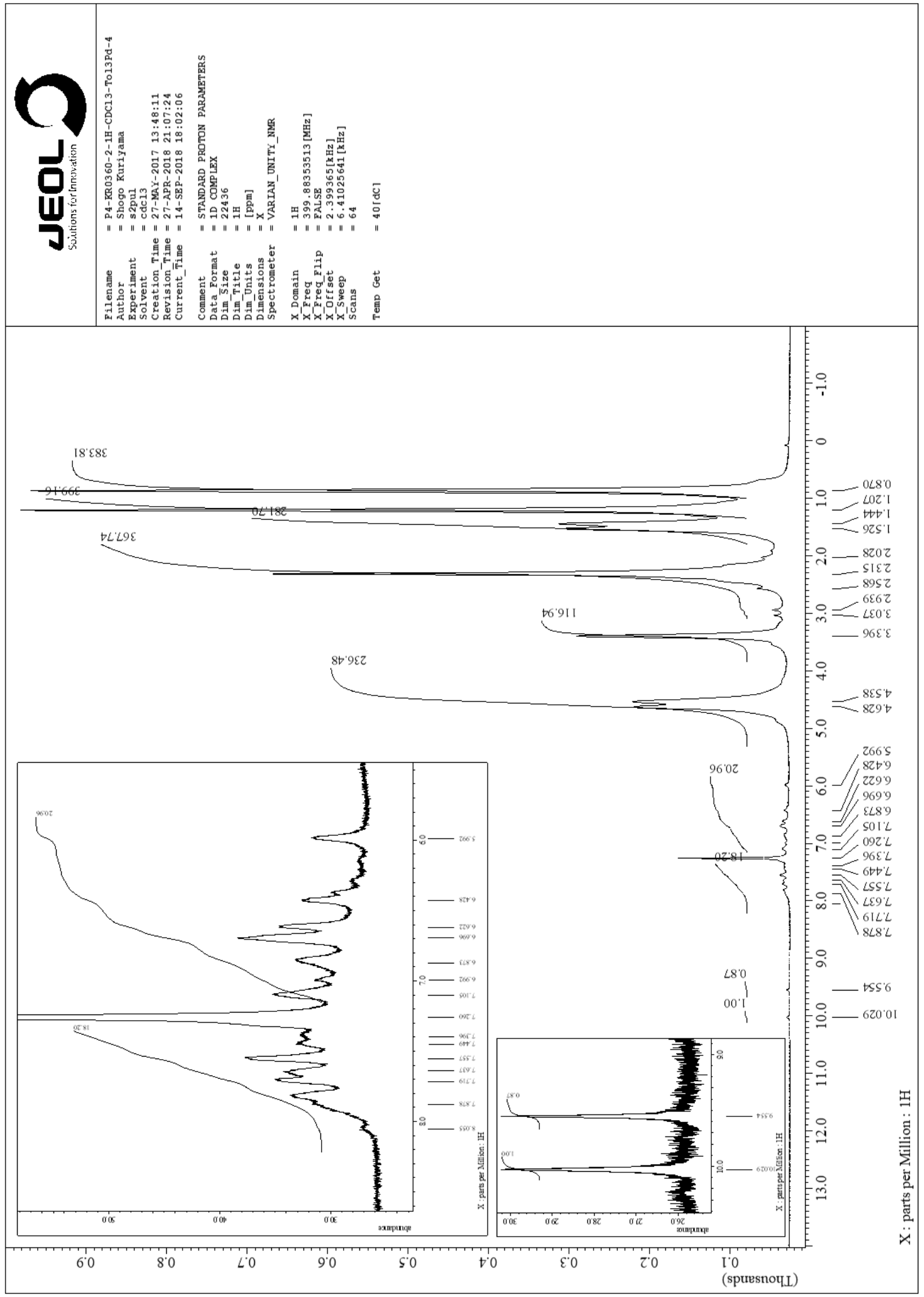

Figure S97. ${ }^{1} \mathrm{H}$ NMR spectrum of $\mathbf{P 2 a}(40)$ in $\mathrm{CDCl}_{3}$. 


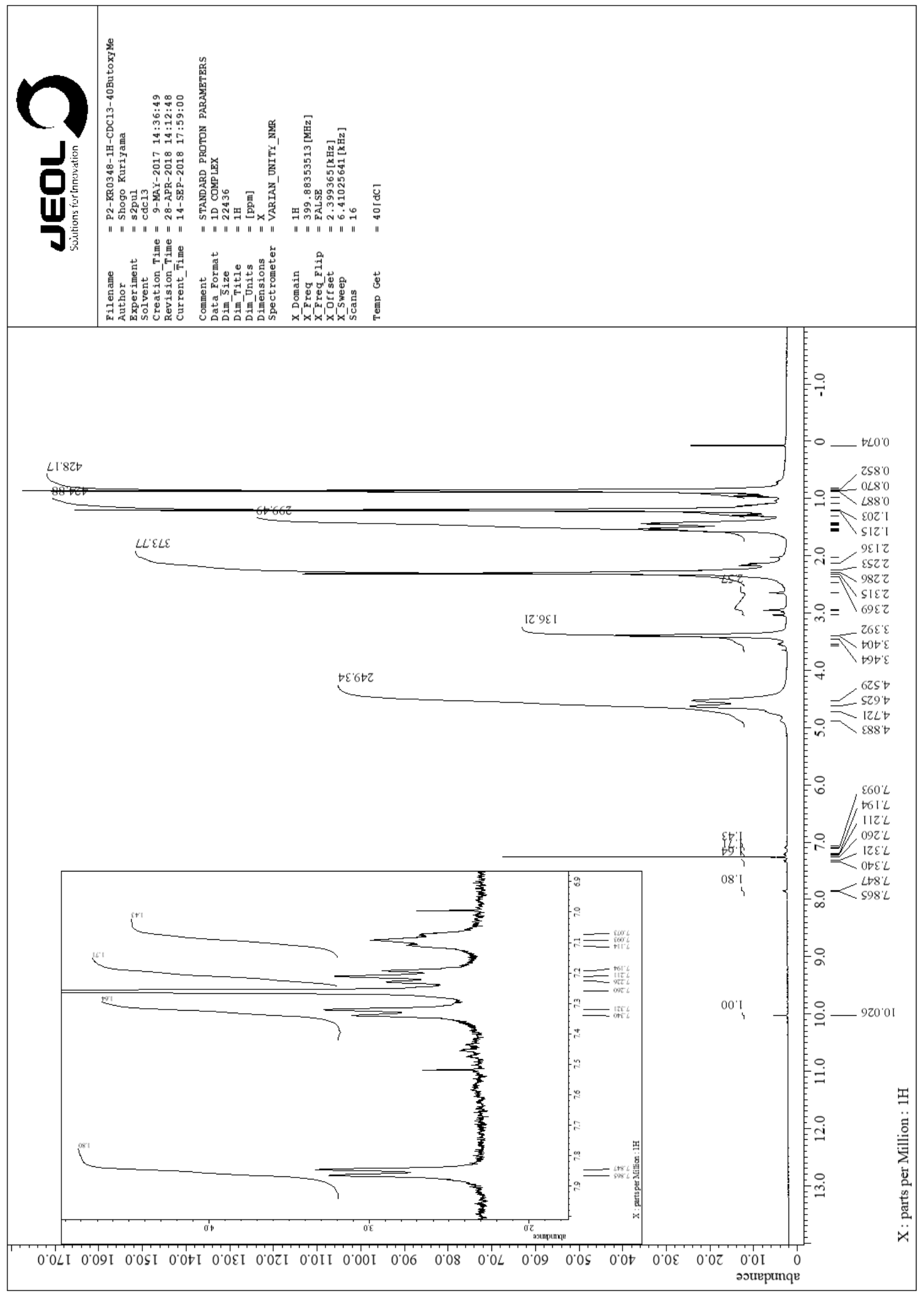

Figure S98. ${ }^{1} \mathrm{H}$ NMR spectrum of $\mathbf{P 4}$ in $\mathrm{CDCl}_{3}$. 


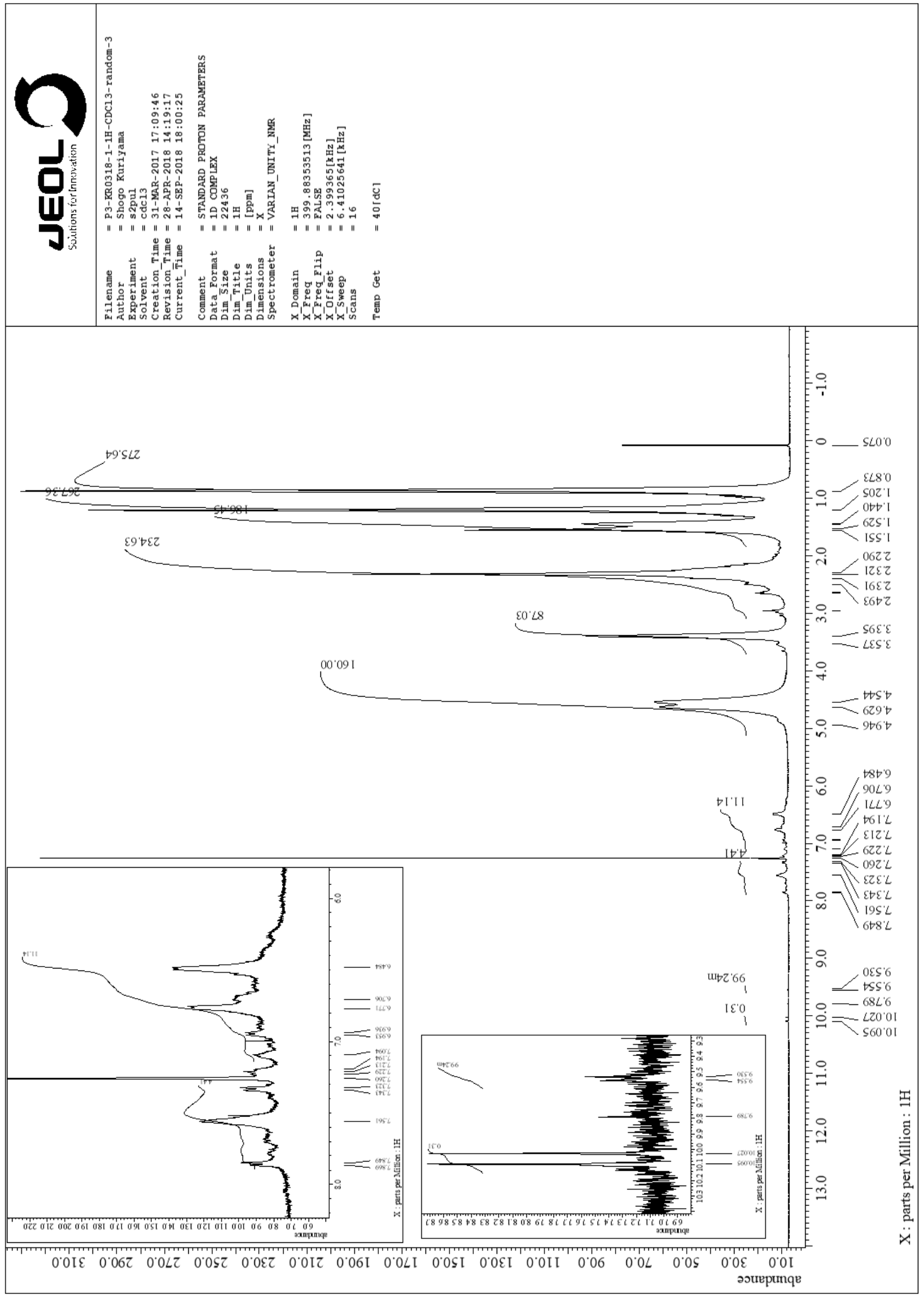

Figure S99. ${ }^{1} \mathrm{H}$ NMR spectrum of $\mathbf{P 5}$ in $\mathrm{CDCl}_{3}$. 


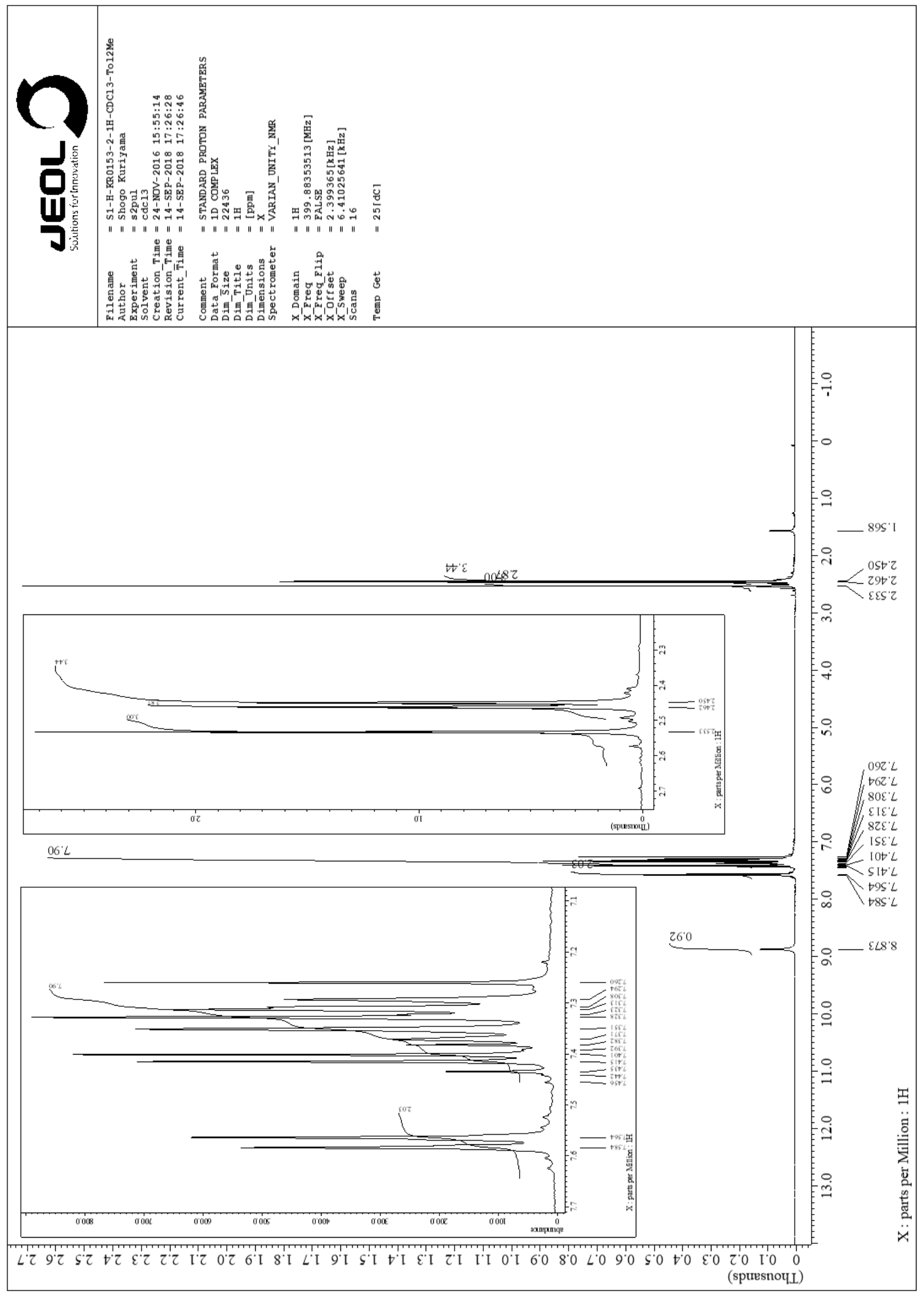

Figure S100. ${ }^{1} \mathrm{H}$ NMR spectrum of $\mathbf{S} 1$ in $\mathrm{CDCl}_{3}$. 


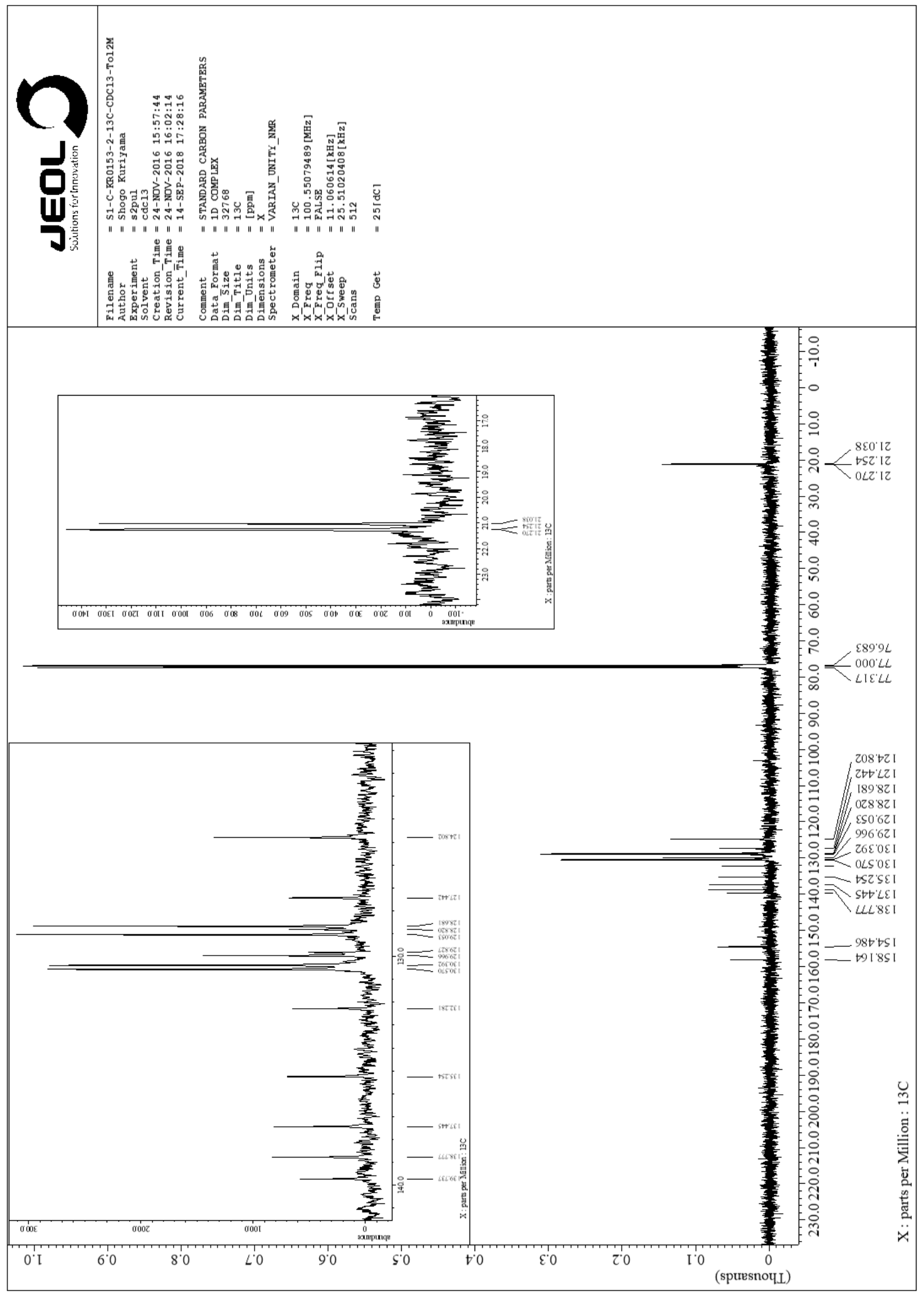

Figure S101. ${ }^{13} \mathrm{C}$ NMR spectrum of $\mathbf{S 1}$ in $\mathrm{CDCl}_{3}$. 


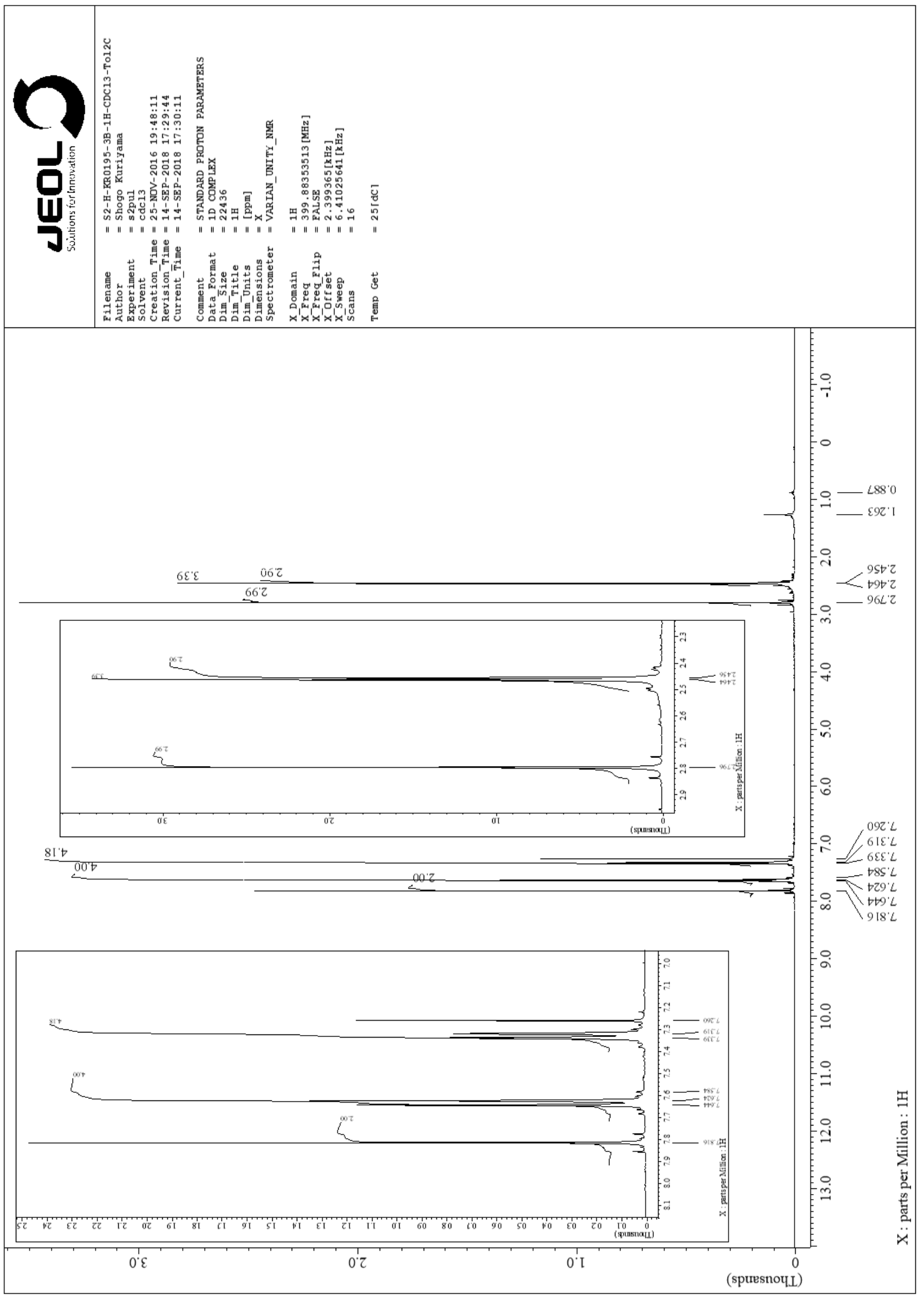

Figure S102. ${ }^{1} \mathrm{H}$ NMR spectrum of $\mathbf{S 2}$ in $\mathrm{CDCl}_{3}$. 


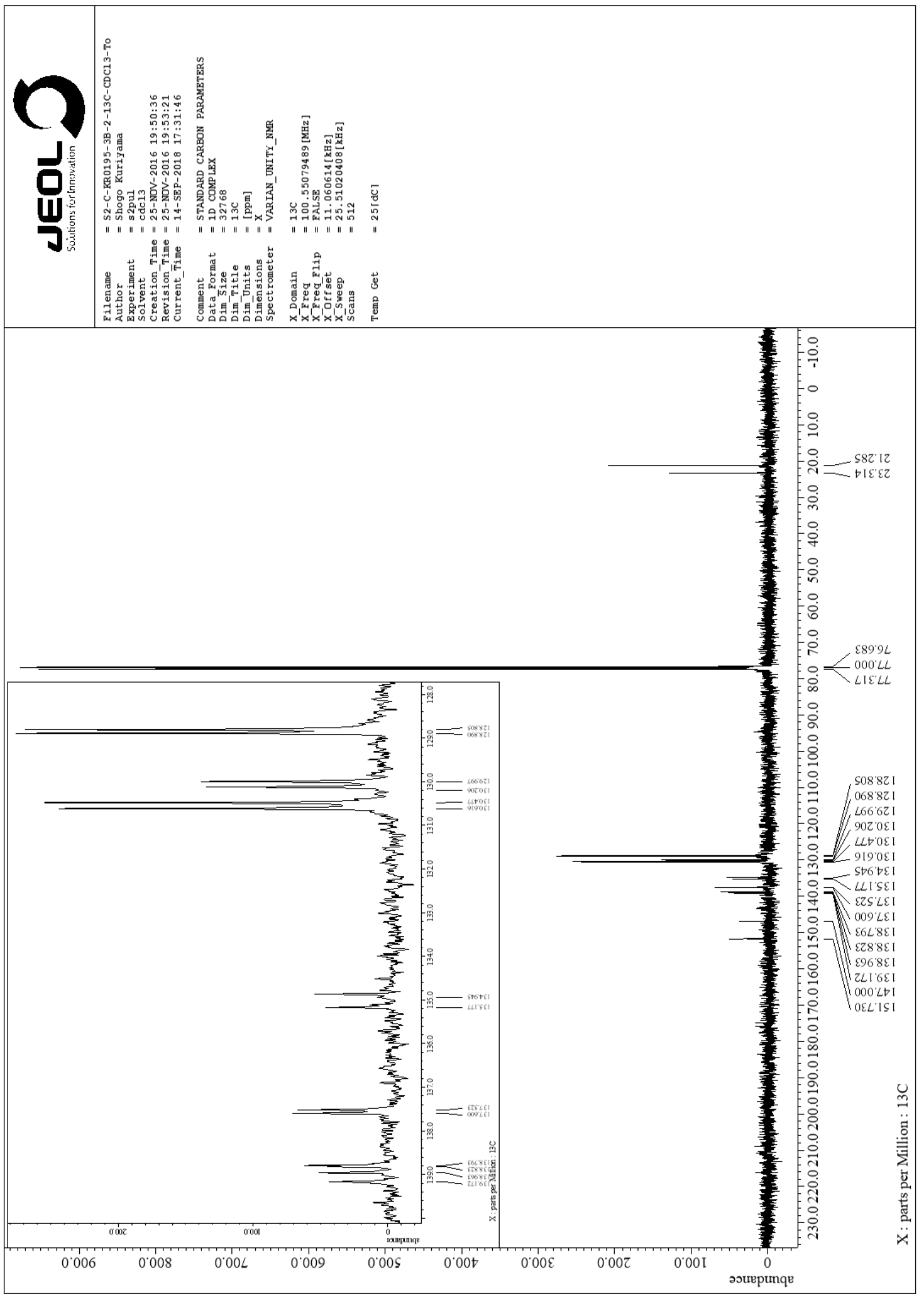

Figure $\mathbf{S 1 0 3} .{ }^{13} \mathrm{C}$ NMR spectrum of $\mathbf{S 2}$ in $\mathrm{CDCl}_{3}$. 


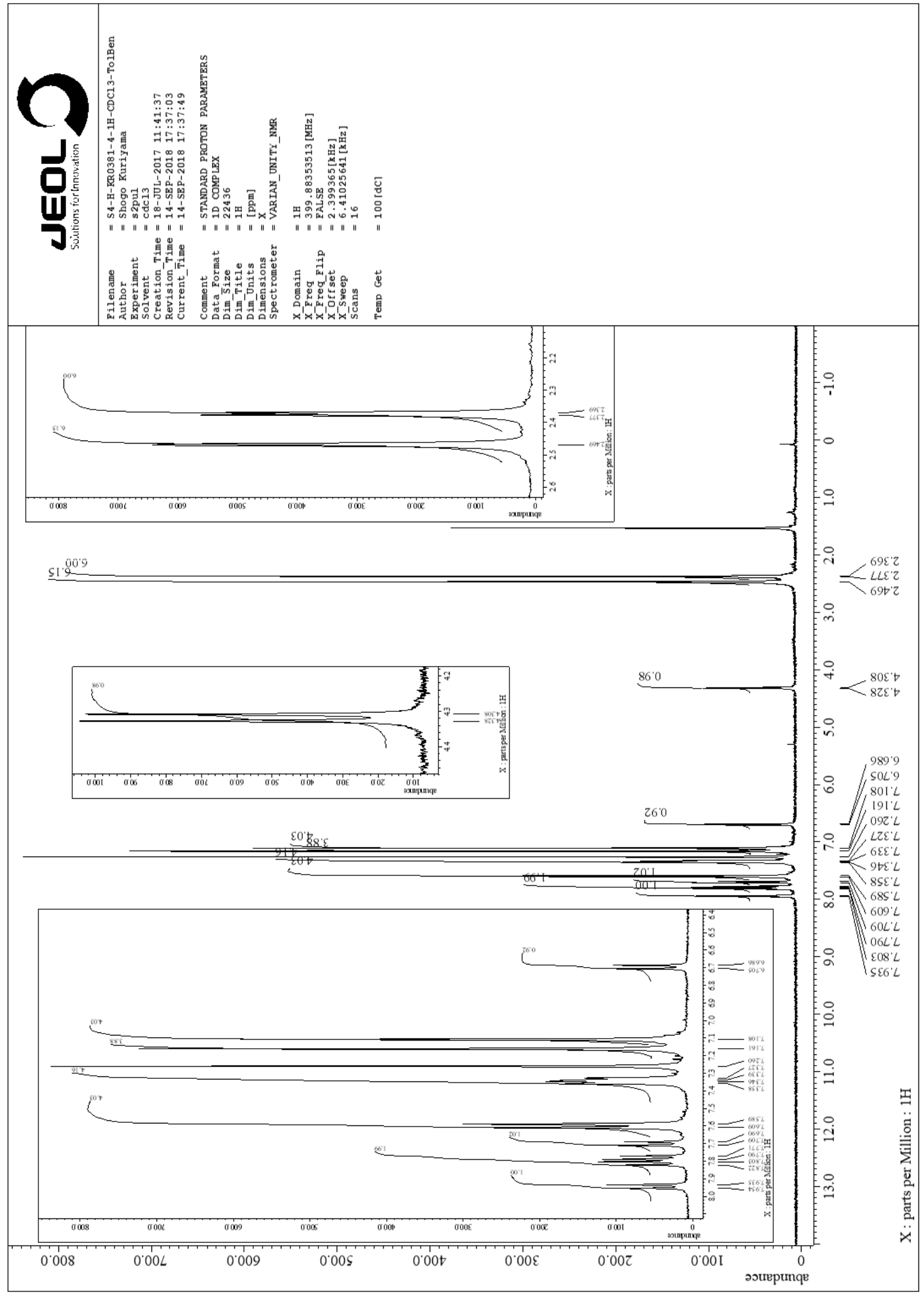

Figure S104. ${ }^{1} \mathrm{H}$ NMR spectrum of $\mathbf{S 3}$ in $\mathrm{CDCl}_{3}$. 


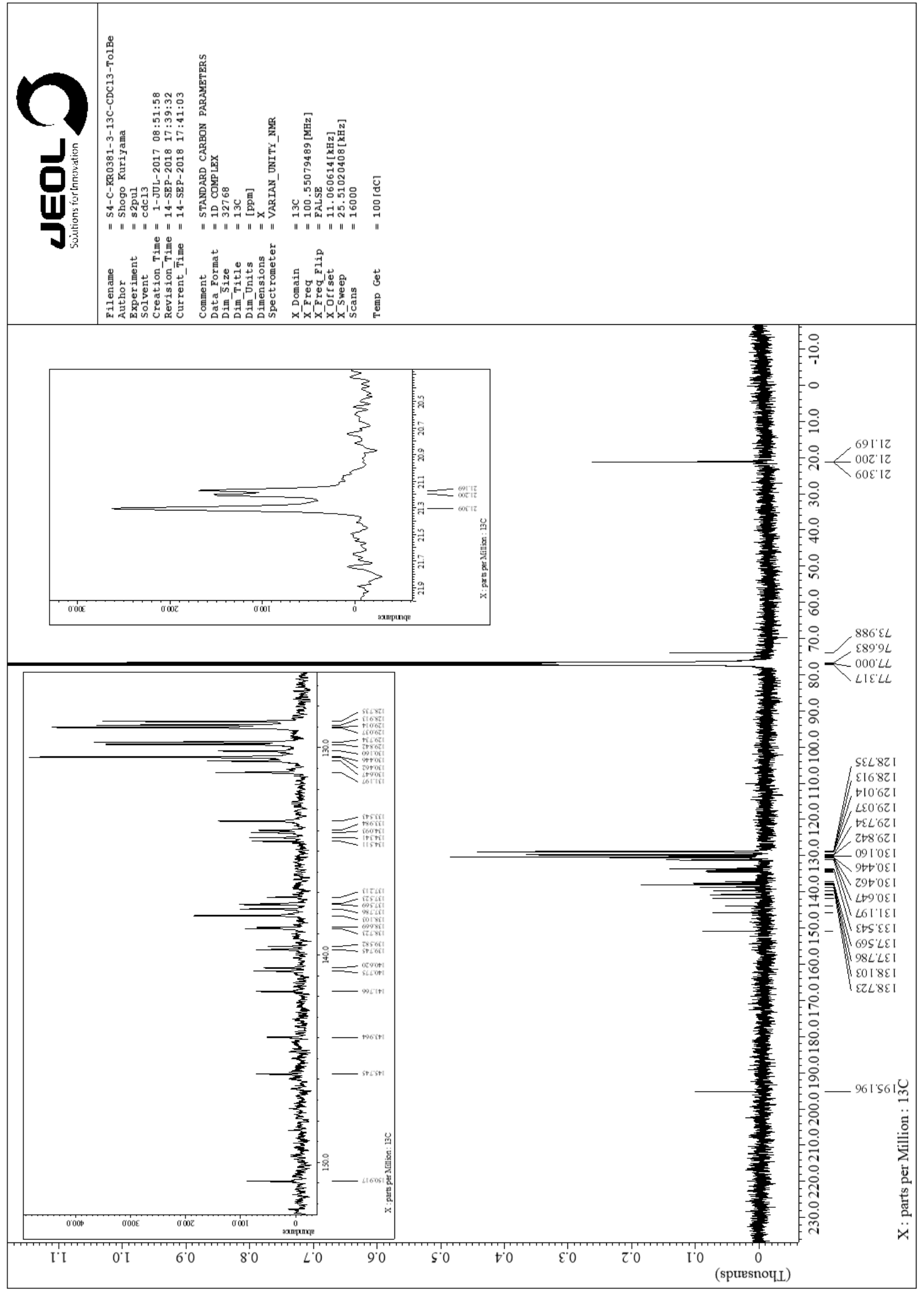

Figure $\mathbf{S 1 0 5} .{ }^{13} \mathrm{C}$ NMR spectrum of $\mathbf{S 3}$ in $\mathrm{CDCl}_{3}$. 